\title{
SEARCH FOR SHORT-BASELINE OSCILLATIONS AT THE NOvA NEAR DETECTOR
}

To be submitted in the partial fulfilment for the degree of DOCTOR OF PHILOSOPHY IN PHYSICS

\author{
BY \\ SIVA PRASAD K \\ 12PHPH17

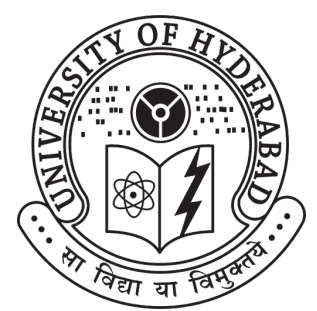 \\ Under the supervision of \\ Prof. BINDU A BAMBAH \\ School of Physics \\ University of Hyderabad \\ Hyderabad 500 046, INDIA
}

April 2018 



\section{DECLARATION}

I hereby declare that, this thesis titled Search For Short-Baseline Oscillations at the NOvA Near Detector submitted by me, under the guidance and supervision of Prof. Bindu A Bambah, is a bonafide research work and is free from plagiarism. I also declare that it has not been submitted previously, in part or in full to this University or any other University or Institution, for the award of any degree or diploma. I hereby agree that my thesis can be deposited in Shodhganga/INFLIBNET.

A report on plagiarism statistics from the University Librarian is enclosed.

Hyderabad

(Siva Prasad K)

Date:

Reg. No.: 12PHPH17 



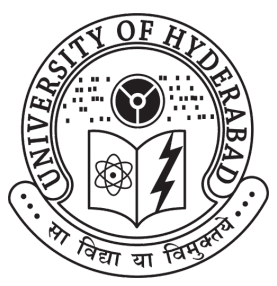

\section{CERTIFICATE}

This is to certify that the thesis entitled Search For Short-Baseline Oscillations at the NOvA Near Detector submitted by Siva Prasad K bearing registration number 12PHPH17 in partial fulfilment of the requirements for award of Doctor of Philosophy in the School of Physics is a bonafide work carried out by him under my and guidance.

This thesis is free from plagiarism and has not been submitted previously in part or in full to this or any other University or Institution for award of any degree or diploma.

Further, the student has the following publications before the submission of the thesis for adjudication.

- Thesis Publications

1. P. Adamson et.al. [NOvA Collaboration], "First measurement of electron neutrino appearance in NOvA", Phys. Rev. Lett. 116, 151806 (2016)

2. P. Adamson et.al. [NOvA Collaboration], "First measurement of muonneutrino disappearance in NOvA", Phys. Rev. D 93051104 (2016)

3. P. Adamson et.al. [NOvA Collaboration], "Constraints on Oscillation Parameters from $\nu_{e}$ Appearance and $\nu_{\mu}$ Disappearance in NOvA", Phys. Rev. Lett. 118, 231801 (2017)

4. P. Adamson et.al. [NOvA Collaboration], "Measurement of the neutrino mixing angle $\theta_{23}$ in NOvA", Phys. Rev. Lett. 118, 151802 (2017)

5. P. Adamson et.al. [NOvA Collaboration], "Search for active-sterile neutrino mixing using neutral-current interactions in NOvA", Phys. Rev. D 96, 072006 (2017), arXiv:1706.04592

6. NOvA Collaboration, "Sterile neutrino search with NOvA Near Detector", to be submitted in mid 2018 to Physical Review Letters.

- Other Publications 
1. Bindu A. Bambah, C. Mukku, T. Shreecharan, K. Siva Prasad, "Entanglement in a model for Hawking radiation: An application of quadratic algebras", Annals of Physics, 330, 201-219 (2013).

- Conference Proceedings

1. Siva Prasad K, Adam Aurisano, Louise Suter, Alex Sousa, Bindu A Bambah, "Search for short-baseline oscillations at the NOvA Near Detector", Proceedings from Neutrino 2016, J.Phys.Conf.Ser. 888 (2017) no.1, 012144 , FERMILAB-CONF-16-426-ND-PPD

2. G.S. Davies, A. Aurisano, G.K. Kafka, S. Kasetti, R. Keloth, A. Sousa, L. Suter and S. Yang, "Searches for Sterile Neutrinos with NOvA", ICHEP 2016 Proceedings, https://pos.sissa.it/282/972/pdf

3. A. Hatzikoutelis, S. Kotelnikov, B. A. Bambah, S. P. Kasetti, "New light weakly-coupled particle searches in a neutrino detector". IOP Journal of Physics: Conference Series 490 (2014) 012070

4. A. Hatzikoutelis, S. Kotelnikov, B. A. Bambah, S. P. Kasetti, "Search for Hidden Sector and Dark Matter Particles Produced at Fermilab's NuMI Target", Proceedings from 10th Patras Workshop on Axions, WIMPs (AXION-WIMP 2014) Conference. FERMILAB-CONF-14-376-PPD

Further, the student has passed the following courses towards fulfilment of coursework requirement for Ph.D:

\begin{tabular}{|c|l|c|c|}
\hline Course Code & Name & Credits & Pass/Fail \\
\hline PY801 & Advanced Quantum Mechanics & 4 & Pass \\
\hline PY803 & Advanced Statistical Mechanics & 4 & Pass \\
\hline PY804 & Advanced Electromagnetic Theory & 4 & Pass \\
\hline PY821 & Research Methodology & 4 & Pass \\
\hline
\end{tabular}

Prof. Bindu A Bambah

Thesis Supervisor

School of Physics

University of Hyderabad
Prof. Bindu A. Bambah

Dean

School of Physics

University of Hyderabad 



\section{Acknowledgements}

First and foremost, I would like to thank is my thesis advisor, Prof. Bindu A Bambah for all the encouragement and motivation she has provided me with throughout the project. I sincerely thank her for giving me the opportunity to perform neutrino research at such a wonderful place like Fermi National Accelerator Laboratory, USA. I thank her for believing in my capabilities as a researcher. I would like to thank my Fermilab advisor, Dr. Louise Suter. Her knowledge about neutrino physics, motivation and support helped me finish this thesis analysis. Anything is less whatever I say about her. I really would thank her patience in advising me and making this analysis successful.

I would like to thank Dr. John Cooper and Dr. Ting Miao for their support at Fermilab. I would like to thank Athanasios Hatzekoutelos who helped me setup while I first came to Fermilab. I would like to thank Dr. Adam Aurisano who helped me get started on my thesis topic analysis. Thank you for being patient and answering any kind of dumb question that I had. I would also like to thank, Dr. Christopher Backhouse, for being my coding inspiration and for helping me understand issues related to software and for laying the foundation for the software. I would like to thank Dr. Gavin Davies for his immense help throughout my analysis.

Sincere thanks to all NOvA collaborators for their tireless effort in making way for analysis.

I would also like to thank Department of Science and Technology, India and Fermilab, USA for making such wonderful collaboration and for giving such opportunities to do research.

I shouldn't forget to thank my best friends, Arun, Navaneeth, Rijeesh and Sijith for their patience to bear with me, with whom I spent most of my dinner time at Fermilab.

My heartfelt thanks to my parents, father Gangadhara Rao, mother Satyanarayanamma, eldest brother Veeranjaneyulu and his family, and Suresh and his family, who helped me get this far in my life and career. I should specially thank my elder brother, Suresh for his unlimited support through out my educational career. Finally my wonderful wife, Mythri without whom, I wouldn't have had the motivation to finish this project.

Finally, I would like to thank everyone whosoever is involved in this effort.

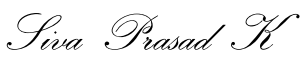





\section{Abstract}

Anomalous results from past neutrino experiments have been interpreted as potential evidence for an additional sterile neutrino with a mass on order of $1 \mathrm{eV}$, but this evidence remains inconclusive. The NOvA Near Detector is a 293 ton almost fully-active finegrained liquid scintillator detector, that was designed for electron-neutrino identification. The detector is placed along the Fermilab NuMI beam line $1 \mathrm{~km}$ from the target. At this off-axis angle the detector is exposed to a narrow band beam peaked at $2 \mathrm{GeV}$. Therefore the NOvA Near Detector will see neutrinos with a L/E range that is sensitive to oscillations between active neutrinos and light sterile neutrinos. In this thesis I discuss NOvA sensitivity from the joint electron-neutrino appearance and muon-neutrino disappearance analysis search for short-baseline sterile neutrino mixing. 


\section{Contents}

$\begin{array}{lll}\text { Declaration } & \text { iii }\end{array}$

Certificate v v

Acknowledgements viii

Abstract $\quad x$

List of Figures $\quad$ xv

List of Tables $\quad$ xxiii

1 Introduction to Neutrino Physics 1

1.1 The Beginning . . . . . . . . . . . . . . . . . . 1

1.2 How can a Neutrino Interact? . . . . . . . . . . . . . . . . . . . 2

1.3 Neutrinos in Standard Model . . . . . . . . . . . . . . . . . . . . . . 3

1.4 Neutrino Mass . . . . . . . . . . . . . . . . . . . . . 3

1.5 Three Generations of Neutrinos . . . . . . . . . . . . . . . . . 5

1.6 Common Source of Neutrinos . . . . . . . . . . . . . . . . . 6

1.7 Neutrinos Oscillations . . . . . . . . . . . . . . . . . . 7

1.7.1 Neutrino Oscillations in Vaccum . . . . . . . . . . . . . . 8

1.7.2 Neutrino Oscillations in Matter . . . . . . . . . . . . . . . . 11

1.8 Experimental Evidence for Neutrino Oscillations . . . . . . . . . . . . . . 12

1.8.1 Present Knowledge of Oscillation Parameters . . . . . . . . . . . . 14

1.8.1.1 Known Parameters . . . . . . . . . . . . . . . . . 14

1.8.1.2 Unknown Parameters . . . . . . . . . . . . . . . . 15

1.9 Sterile Neutrinos . . . . . . . . . . . . . . . . . . . . . . . 16

$1.10(3+1)$ Model . . . . . . . . . . . . . . . . . . . . . 16

$1.10 .1 \nu_{e}$ Appearance and $\nu_{\mu}$ Disappearance Probability . . . . . . . . . 20

1.11 Experimental Evidence For Short-Baseline Oscillations . . . . . . . . . . . 21

1.11.1 LSND and MiniBooNE Anomalies . . . . . . . . . . . . . . 21

1.11 .2 MiniBooNE Anomaly . . . . . . . . . . . . . . . . . . . . . . . . . 22

1.11 .3 GALLIUM Anomaly . . . . . . . . . . . . . . . . . . . . . . . . . . . . . . 24

1.11 .4 Reactor Anomaly . . . . . . . . . . . . . . . . . . . 26 
1.11.5 Current Status of $(3+1)$ Oscillation Parameters . . . . . . . . . . . 27

1.11.6 Searching of a Sterile neutrino with NOvA . . . . . . . . . 28

2 NOvA Experiment 31

2.1 Physics Goals . . . . . . . . . . . . . . . . . . 31

2.1 .1 Precise Measurement of $\theta_{23} \ldots \ldots \ldots \ldots$. . . . . . . . 32

2.1 .2 Measurement of $\delta_{C P} \ldots \ldots \ldots \ldots \ldots \ldots$

2.1.3 Resolving Mass Ordering . . . . . . . . . . . . . . . . 32

2.2 NOvA Neutrino Beam . . . . . . . . . . . . . . . . . . . . 33

2.2.1 Making $120 \mathrm{GeV}$ Proton Main Injector Beam . . . . . . . . . . 33

2.2 .2 Making a Neutrino Beam . . . . . . . . . . . . . . . . 34

2.3 NOvA Detectors . . . . . . . . . . . . . . . . . . 37

2.3.1 Two detector Principle . . . . . . . . . . . . . . . . . . . 37

2.3 .2 NOvA Detector Design . . . . . . . . . . . . . . . 37

2.3.2.1 PVC Cell . . . . . . . . . . . . . . 38

2.3.2.2 Liquid Scintillator . . . . . . . . . . . . . . . 38

2.3.2.3 Wavelength Shifting Fiber . . . . . . . . . . . . . 39

2.3.2.4 Avalanche Photo Diode . . . . . . . . . . . . . . . . 40

2.3.2.5 Front-end Electronics Box . . . . . . . . . . . . . . 41

2.3.2.6 Data Concentrator Module . . . . . . . . . . . . . 43

2.4 Data Acquisition System . . . . . . . . . . . . . . . . . . . 44

2.4.1 Timing Synchronization . . . . . . . . . . . . . . . . . 45

2.4 .2 Display Visualizer . . . . . . . . . . . . . . . . . 46

3 Monte Carlo Simulation $\quad 49$

3.1 Overview of Monte Carlo Simulation . . . . . . . . . . . . . . . . 49

3.2 Neutrino Flux . . . . . . . . . . . . . . . . . . . . . . 50

3.3 Particle Propagation . . . . . . . . . . . . . . . . 51

3.4 Neutrino Interactions . . . . . . . . . . . . . . . . . . . . . . 51

3.5 Photon Transport . . . . . . . . . . . . . . . . . . . . . 52

3.6 Electronic Simulation _. . . . . . . . . . . . . . . 53

3.7 Monte Carlo Simulation Tuning . . . . . . . . . . . . . . . . . . 53

3.8 Cherenkov Light . . . . . . . . . . . . . . . . . . . 55

4 Event Reconstruction and Paticle Identification $\quad 59$

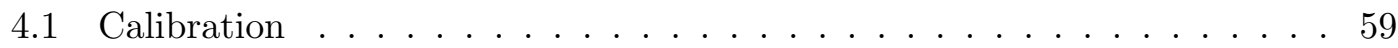

4.1 .1 Attenuation Calibration . . . . . . . . . . . . . . . 59

4.1.2 Absolute Calibration . . . . . . . . . . . . . . . . . . . 61

4.2 Event Reconstruction . . . . . . . . . . . . . . . . . . . . 62

4.2 .1 Clustering Algorithm . . . . . . . . . . . . . . . . . . . . . 63

4.2.2 Line Finding Algorithm - Multi-Hough Transform . . . . . . . . . 64

4.2 .3 Vertex Identification . . . . . . . . . . . . . . . . . . . 65

4.2 .4 Cluster-Vertex Matching . . . . . . . . . . . . . . . . . . 66

4.3 Particle Identification - Convolutional Visual Networks . . . . . . . . . . . 67

4.4 Energy Reconstruction . . . . . . . . . . . . . . . . . . . . . . . 69

4.4.1 $\nu_{e}$ Energy Estimator . . . . . . . . . . . . . . . . . 70

$4.4 .2 \nu_{\mu}$ Energy Estimator . . . . . . . . . . . . . . . . 72 
4.4.2.1 Muon Energy . . . . . . . . . . . . . . . . . . . 73

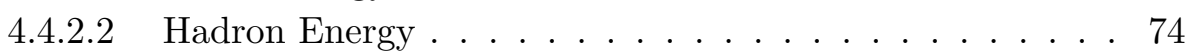

4.4.2.3 Muon Neutrino Energy . . . . . . . . . . . . . . 75

4.4 .3 Track Reconstruction . . . . . . . . . . . . . . 75

5 Systematic Uncertainties $\quad \mathbf{7 9}$

5.1 Systematics Methodology . . . . . . . . . . . . . . . . . . . 79

5.2 Summary of Systematics . . . . . . . . . . . . . . . . . . . . . . . . . . . . . . . . 80

5.3 Flux Systematics . . . . . . . . . . . . . . . . . . . . . . . . . . . . . . . . . 81

5.4 Cross-section Model Systematics . . . . . . . . . . . . . . . . 83

5.4 .1 Neutral Currents . . . . . . . . . . . . . . . . 87

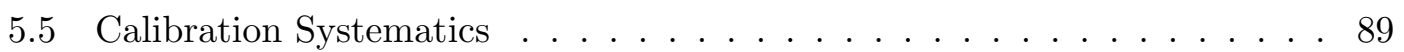

5.6 Rock Systematics . . . . . . . . . . . . . . . . . . . . . . . . . . . . . . . . . . . . . . . . . . . . . .

5.7 Light Level And Cherenkov Systematics . . . . . . . . . . . . . . . . . . . . 104

5.8 Energy Bias . . . . . . . . . . . . . . . . . . . . . . . 105

5.9 Muon Energy Scale . . . . . . . . . . . . . . . . . . . . . . . . . 108

5.10 Normalization Systematics . . . . . . . . . . . . . . . . . . . 108

5.10 .1 POT Accounting . . . . . . . . . . . . . . . . . . . . . . . . . . . . . . . . . . . . . . . . .

5.10 .2 Detector Mass . . . . . . . . . . . . . . . . . . . 108

5.10 .3 Detector Acceptance . . . . . . . . . . . . . . . . . . . 109

5.11 Selection Efficiency of overlaid swapped $\nu_{e} \ldots \ldots \ldots$. . . . . . . . 110

5.12 Negligible systematic effects . . . . . . . . . . . . . . . . . . 110

5.12.1 Periodic Calibration Triggers: Detector Noise Mismodeling . . . . 111

5.12 .2 Intensity Effect . . . . . . . . . . . . . . . . . . 112

6 Analysis $\quad 115$

6.1 SBL Oscillation Analysis Overview f . . . . . . . . . . . . . 116

6.2 New Tools Developed For SBL Analysis . . . . . . . . . . . . . . . . . 118

6.2.1 CAF additions . . . . . . . . . . . . . . . . 118

6.2 .2 Overlaid ND $\nu_{e}$ Appearance Sample . . . . . . . . . . . . . . . 120

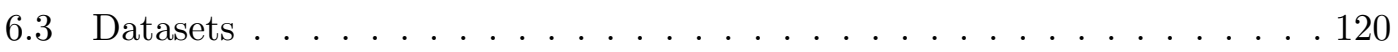

6.4 Event Selection . . . . . . . . . . . . . . . . . . . . . . . 121

6.4.1 Beam and Quality cuts . . . . . . . . . . . . 121

$6.4 .2 \nu_{e}$ Selection Cuts . . . . . . . . . . . . . . . . . . . 122

$6.4 .3 \nu_{\mu}$ Selection Cuts . . . . . . . . . . . . . . . . . . . . . . . . . . . . . . . . . . . .

6.5 Selected events . . . . . . . . . . . . . . . . . . . . 124

6.5.1 Expected signal . . . . . . . . . . . . . . . . . . 125

6.6 Details of fitting framework . . . . . . . . . . . . . . 126

6.6 .1 Log-Likelihood Fitting . . . . . . . . . . . . . . . . . . . . . . . . . . . . . . . . . . . . .

6.6 .2 Joint fit versus Single fit . . . . . . . . . . . . . . . . . . . . . . . . . . . . . . . . . . .

6.7 Analysis Deliverables . . . . . . . . . . . . . . . . . 127

6.7.1 90\% CL limits $\theta_{14}$ as a function of $\Delta \mathrm{m}_{41}^{2} \ldots \ldots \ldots \ldots \ldots \ldots$

6.7.2 90\% CL limits $\theta_{24}$ as a function of $\Delta \mathrm{m}_{41}^{2} \ldots \ldots \ldots \ldots \ldots \ldots$

6.7.3 Sensitivity for $\theta_{\mu e}$ as a function of $\Delta \mathrm{m}_{41}^{2} \ldots \ldots \ldots \ldots . \ldots 129$

6.8 Mock Data Challenges . . . . . . . . . . . . . . . . . . . . . . . 129

6.8.1 Mock Data Sensitivity with no signal injection . . . . . . . . . . 129

6.8.2 Mock Data Sensitivity with LSND signal injection . . . . . . . . 130 
6.9 Studies using data . . . . . . . . . . . . . . . . . . . . . . . . . . . . . . . 132

6.9.1 Near Detector Data and MC Comparison . . . . . . . . . . . . . 132

6.9 .2 Blinding Study . . . . . . . . . . . . . . . . . . . . . . 134

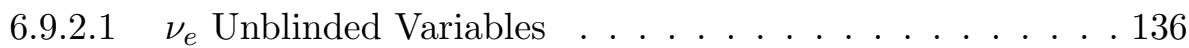

$6.9 .2 .2 \nu_{\mu}$ Unblinded Variables . . . . . . . . . . . . . . . . . . . . . . . . . . . . . .

6.9.3 Muon Removed Electron Events . . . . . . . . . . . . . . . . . . 136

7 Conclusion and Future 143

$\begin{array}{lr}\text { A Samples Used } & \mathbf{1 4 5}\end{array}$

A.1 Systematic Samples . . . . . . . . . . . . . . . . . . . . . . 145

B New Tools Developed For SBL Analysis 147

B.1 CAFAna Framework . . . . . . . . . . . . . . . . . . . . . . . . . . . . . . . . . . . . .

B.1.1 NDOscCurve . . . . . . . . . . . . . . . . . . . 147

B.1.2 NDOscillatableSpectrum . . . . . . . . . . . . . . . . . . . . . . . . . . . . . . . . . . . . . . . . . . . . . .

B.1.3 NDExtrap . . . . . . . . . . . . . . . . . . . . . . . . . 148

B.1.4 NDPredictionExtrap . . . . . . . . . . . . . . . . . . . . 148

B.1.5 PredictionInterpJointAna . . . . . . . . . . . . . . . 149

$\begin{array}{lr}\text { C GENIE Systematic Tables } & 151\end{array}$

C.1 GENIE Systematic Table For $\nu_{e}$ Selected Sample . . . . . . . . . . . . . . 151

C.2 GENIE Systematic Table For $\nu_{\mu}$ Selected Sample . . . . . . . . . . . . . . 154

C.3 Effect of GENIE Parameters on Energy Distribution . . . . . . . . . . . 157 


\section{List of Figures}

1.1 The Feynman diagrams for the weak neutrino charged current (left) and neutral current (right). . . . . . . . . . . . . . 4

1.2 Shows the various processes involved the p-p cycle inside a star. Neutrinos emitted almost in every reaction to carry away some of the energy. This figure is taken from Carlo Giunti and Chung W. Kim [18] . . . . . . . . 6

1.3 Shows the various processes involved in the generation of neutrinos in the upper atmosphere. This process happens at an altitude of $\approx 30 \mathrm{~km}$. Figure taken from Los Alamos Science 25 (1997) [19]. . . . . . . . . . . 7

1.4 Left: Shows the nuclear fission reaction chain. Right: To attain the stability, nuclei undergo several decays emitting electons and anti-electron neutrinos $[20] \ldots \ldots \ldots \ldots \ldots$. . . . . . . . . . . . . . . 8

1.5 Shows the generation of neutrino in NuMI facility at Fermilab. . . . . . 8

1.6 The two possible mass orderings of the masses in neutrino 3 -flavor model. The color shows the makeup of mass eigen states by the flavour eigen states. 10

1.7 Energy spectra of neutrino flux from the pp and CNO chains as predicted by the Standard Solar Model [48]. For continuos sources, the differential flux is measured in $\mathrm{cm}^{-2} \mathrm{~s}^{-1} \mathrm{MeV}^{-1}$ and for the lines, it is measured in $\mathrm{cm}^{-2} \mathrm{~s}^{-1}$. This figure is taken from $[49] . \ldots \ldots . \ldots . \ldots 13$

$1.8 \quad(3+1)$ flavor model . . . . . . . . . . . . . . . . . . . . 17

1.9 Left: Shows the energy distribution of the events selected by LSND. Right: Shows the $L / E$ distribution of the selected events. Ref. [76]. . . . . . . . 23

1.10 Shows LSND results in the $\left(\sin ^{2} 2 \theta, \Delta \mathrm{m}^{2}\right)$ plane at $90 \%$ and $99 \%$ C.L. . . 23

1.11 Left: Shows the neutrino flux in the neutrino mode at MiniBooNE; Right: Shows the neutrino flux in the anti neutrino mode. Ref. [77]. . . . . . . . 24

1.12 Left: Shows the selected event rates in the neutrino mode at MiniBooNE. Right: Shows the selected event rates in the anti-neutrino mode. The

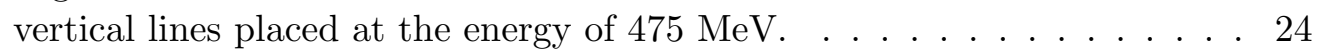

1.13 Shows the results in the $\left(\sin ^{2} 2 \theta, \Delta \mathrm{m}^{2}\right)$ plane at $90 \%$ and $99 \%$ C.L. As we see MiniBooNE in the neutrino modes excludes some of the LSND region at various significance levels. Also, shown the results from KARMEN [81]. 25

1.14 Shows the combined results from GALLEX and SAGE experiments in the $\left(\sin ^{2} 2 \theta, \Delta \mathrm{m}^{2}\right)$ plane at various C.L. Ref. [34-43]. . . . . . . . . . 27

1.15 Shows the ratio between measured and expected neutrino flux from various short-baseline reactor neutrino experimnets. Ref. [86] . . . . . . . . 28

1.16 Shows the allowed regions in the $\left(\sin ^{2} 2 \theta, \Delta \mathrm{m}^{2}\right)$ plane combining (global fit) all short-baseline reactor neutrino experiments, GALLEX, SAGE and MiniBooNE new results after considering the correlations between these experiments. Ref. [86]. . . . . . . . . . . . . . . . . 29 
1.17 The allowed regions for the $(3+1)$ mixing elements using the PrGlo17 global fit. Ref. [88].

1.18 The results from the global fit using the $(3+1)$ model from GLoBES. The last column PrGlo17 gives the current best-fit values which didn't include the MiniBooNE low energy excess into the gloabl fit. Otherwise, all short-baseline reactor experiments, MINOS, IceCube, NEOS results included into the fit. Ref. $[88] . \ldots \ldots \ldots$. . . . . . . . . .

2.1 A cartoon of making neutrinos at Fermilab. . . . . . . . . . . . . 33

2.2 A cartoon of the NuMI beamline . . . . . . . . . . . . . . . . 34

2.3 The distribution of energy for pions (left) and neutrinos (right) at different off-axis angles. . . . . . . . . . . . . . . . 35

2.4 The true energy distribution of $\nu_{\mu}$ 's shown in left and of $\nu_{e}$ 's shown in right at the NOvA Near Detector. For $\nu_{\mu}$ 's coming from Kaons are shown in blue, from Pions shown in red, and the total is shown in black. For $\nu_{e}$ 's coming from Kaons are shown in red, from Muons shown in blue, and the total is shown in black. . . . . . . . . . . . . . . . . .

2.5 The true length distribution of $\nu_{\mu}$ 's shown in left and of $\nu_{e}$ 's shown in right at the NOvA Near Detector. For $\nu_{\mu}$ 's coming from Kaons are shown in blue, from Pions shown in red, and the total is shown in black. For $\nu_{e}$ 's coming from Kaons are shown in red, from Muons shown in blue, and the total is shown in black. . . . . . . . . . . . . . . .

2.6 The true L over true E energy distribution of $\nu_{\mu}$ 's shown in left and of $\nu_{e}$ 's shown in right at the NOvA Near Detector. For $\nu_{\mu}$ 's coming from Kaons are shown in blue, from Pions shown in red, and the total is shown in black. For $\nu_{e}$ 's coming from Kaons are shown in red, from Muons shown in blue, and the total is shown in black. . . . . . . . . . . . . . 36

2.7 NuMI flux at the NOvA Near Detector as simulated with FLUKA showing

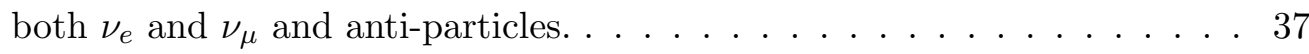

2.8 NOvA Design Criteria. . . . . . . . . . . . . . . . . . . . . . 38

2.9 The NOvA detector basic unit, single PVC cell. . . . . . . . . . . . . . . 39

2.10 Shows how the wavelength shifters shifts the wavelength. This figure is taken NOvA technical design report $[89] . \ldots . . . . . . . . . .441$

2.11 The absorption and emission profile of K27 fluorescent dye in the fiber This figure is taken NOvA technical design report [89] . . . . . . . . . . 42

2.12 A NOvA Avalanche Photo-Diode (left), a diagram of the function of a APD (right). . . . . . . . . . . . . . . . . 42

2.13 Front End Board used in NOvA and it's connections to the APD. . . . . . 43

2.14 Layout of the FEB. Major components are the carrier board connector location at the left, which brings the APD signals to the NOvA ASIC, which performs integration, shaping, and multiplexing. The chip immediately to the right is the ADC to digitize the signals, and FPGA for control, signal processing, and communication. . . . . . . . . . . . 43

2.15 Block diagram of a Data Concentrator Module. . . . . . . . . . . . . . . . 44

2.16 Data Acquisition System in NOvA. . . . . . . . . . . . . . . . . . . 45

2.17 Timing distribution system in NOvA. . . . . . . . . . . . . . . 46

2.18 This is the event display of the selected appeared $\nu_{e}$ candidate at the Far Detector. 
3.1 The light collection rate by WLS fiber in NOvA using the ray tracing simulation. . . . . . . . . . . . . . . . 52

3.2 The data amd MC simulation comparison for the number of photo-electrons for both ND (left) and FD (right). As we see, both agree well in the peak region. . . . . . . . . . . . . . . . . . 54

3.3 APD sag as seen in a single APD. These are the simulated readout traces. The readout trace shown in black is for channel which had no energy deposit, but exhibitibg APD sag due to red traces of other channels. Refer [97]. . . . . . . . . . . . . . . . . . . 54

3.4 Shows the number of Cherenkov photons emitted as a function of wavelength at different energy parameter $\beta$. The red shaded band is the abosrption range of k-27 coated on the walls of PVC cell. Refer [101]. . . 56

3.5 Shows the absorption length with the wavelength for the NOvA scintillator. Refer [101] . . . . . . . . . . . . . . . . 56

3.6 Shows the number of Cherenkov photons emitted with energy for muons (green), electron (blue) and protons (red). Refer [101]. . . . . . . . . 57

4.1 An example of a NOvA tricell. . . . . . . . . . . . . . . 60

4.2 The full attenuation fit for ND (left) and FD (right) including the LOWESS fit from data. The blue curve is the full attenuation fit and red curve is the double exponential fit. . . . . . . . . . . . . . . . . . . 61

4.3 The $\mathrm{dE} / \mathrm{dx}$ of a stopping muon measured in FD cosmic data as a function of distance from the end of the track. . . . . . . . . . . . . 62

4.4 This plot shows the attenutaion corrected response in $\mathrm{dE} / \mathrm{dx}$ for both near detector (top) and far detector (bottom). The difference between the data and $\mathrm{MC}$ is used to find a scale factor for the conversion of PECorr $/ \mathrm{cm}$ to $\mathrm{MeV} / \mathrm{cm} \ldots \ldots \ldots \ldots \ldots \ldots \ldots$

4.5 The performance of Slicer4D module is quantified in terms of function of Figure of Merit (FOM), is the ratio of signal to square root of total selected events, is shown for ND (left) and FD (right). . . . . . . . . . 64

4.6 The Hough lines after the four iterations (left) and the Hough space $(\rho, \theta)$

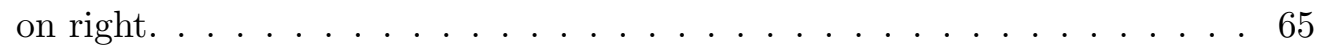

4.7 Shows the NOvA CVN architecture. . . . . . . . . . . . . . 70

4.8 The example NOvA CVN applied to a $\nu_{\mu}$ CC interaction. . . . . . . . . 71

4.9 The relationship between electromagnetic component energy and the hadronic component of energy for the Near Detector. . . . . . . . . . . . . . 72

4.10 The energy resolution using $\nu_{e}$ energy estimator for the Near Detector. . . 72

4.11 The energy resolution as a function of true enutrino energy using $\nu_{e}$ energy estimator for the Near Detector. We clearly see that energy resolution is independent of true energy. . . . . . . . . . . . . . . 73

4.12 The 2D histogram of reconstructed muon length vs true muon energy (left). A gaussian fit is performed for the true energy of muons at every vertical slice. . . . . . . . . . . . . . . . . . . . 74

4.13 A multi linear fit is performed to find the peak of gaussian profile at every vertical slice. The position of dotted lines are where the each spline ends (left). The energy resolution of muon after the fit (right).

4.14 The 2D histogram of reconstructed muon length vs true muon energy (left). A gaussian fit is performed for the true energy of muons at every vertical slice (right). . . . . . . . . . . . . . . 75 
4.15 A multi line fit is performed to find the peak of gaussian profile at every vertical slice. The position of dotted lines are where the each spline ends (left). The energy resolution of hadron after the fit (right). . . . . . . . 75

4.16 Neutrino true energy versus reconstructed energy. . . . . . . . . . . . 76

4.17 The resolution of the neutrino energy. . . . . . . . . . . . . 76

5.1 Shows effect of all systematics on $\nu_{e}$ prediction (left) and $\nu_{\mu}$ prediction

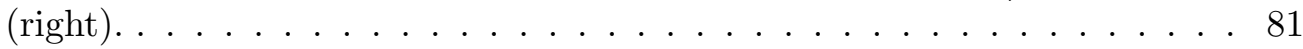

5.2 Effect of all systematics on $\theta_{\mu e}$ sensitivity. . . . . . . . . . . . . . . 82

5.3 Shows the $\nu_{e}$ prediction (left) and $\nu_{\mu}$ prediction (right) with beam transport systematics. . . . . . . . . . . . . . . . . 83

5.4 Shows the $\nu_{e}$ prediction (left) and $\nu_{\mu}$ prediction (right) with PPFX systematics with five principal components. . . . . . . . . . . . 83

5.5 Shows the shifted $\nu_{e}$ prediction (left) and $\nu_{\mu}$ prediction (right) with ND specific PPFX systematics with five principal components. . . . . . . . . 84

5.6 Effect of beam transport (left) and PPFX (right) systematics on $\theta_{\mu e}$ sen-

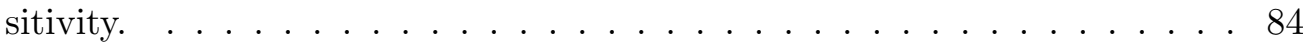

5.7 Showing the effect of all large cross-section systematics having more than $2 \%$ effect on both $\nu_{e}$ prediction (left) and $\nu_{\mu}$ prediction (right). . . . . 8 87

5.8 Showing the effect of summed small cross-section systematics on both $\nu_{e}$

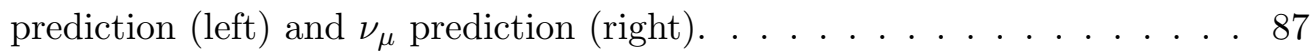

5.9 Effect of GENIE systematics on the $\theta_{\mu e}$ sensitivity at $90 \%$ C.L. using joint fit (red) as compared to black which has no systematics included. The left plot shows the effect of summed small GENIE and the right plot shows the effect of large GENIE parameters. . . . . . . . . . . . . . . 88

5.10 Shows the effect of GENIE parameter RPAShapeenh2017 (left) and MECq0Shape (right) $\nu_{e}$ selected sample which doesn't include appeared $\nu_{e}$ 's. The effect of large genie parameters on the both $\nu_{e}$ and $\nu_{\mu}$ selected spectra is shown in Table 5.3 and $5.4 \ldots \ldots \ldots \ldots$. . . . . . . . . . . . 90

5.11 Shows the effect of GENIE parameter MECEnuShape (left) and DISvnCC1pi (right) $\nu_{e}$ selected sample which doesn't include appeared $\nu_{e}$ 's. The effect of large genie parameters on the both $\nu_{e}$ and $\nu_{\mu}$ selected spectra is shown in Table 5.3 and $5.4 \ldots$. . . . . . . . . . . . . . . . . . 90

5.12 Shows the effect of GENIE parameter MaCCRES (left) and MvCCRES (right) $\nu_{e}$ selected sample which doesn't include appeared $\nu_{e}$ 's. The effect of large genie parameters on the both $\nu_{e}$ and $\nu_{\mu}$ selected spectra is shown in Table 5.3 and $5.4 \ldots \ldots \ldots$. . . . . . . . . . . . . . . 91

5.13 Shows the effect of GENIE parameter MaCOHpi (left) and R0COHpi (right) $\nu_{e}$ selected sample which doesn't include appeared $\nu_{e}$ 's. The effect of large genie parameters on the both $\nu_{e}$ and $\nu_{\mu}$ selected spectra is shown in Table 5.3 and $5.4 \ldots \ldots \ldots \ldots$. . . . . . . . . . . . . . . . . . . . .

5.14 Shows the effect of GENIE parameter MFP_N on $\nu_{e}$ selected sample which doesn't include appeared $\nu_{e}$ 's. The effect of large genie parameters on the both $\nu_{e}$ and $\nu_{\mu}$ selected spectra is shown in Table 5.3 and 5.4 . . . . . . 92

5.15 Shows the effect of GENIE parameter RPAShapeenh2017 (left) and MECEnuShape (right) $\nu_{\mu}$ selected sample which doesn't include appeared $\nu_{e}$ 's. The effect of large genie parameters on the both $\nu_{e}$ and $\nu_{\mu}$ selected spectra is shown in Table 5.3 and $5.4 \ldots \ldots \ldots \ldots \ldots \ldots$ 
5.16 Shows the effect of GENIE parameter DISvpCC2pi (left) and DISvnCC1pi (right) on $\nu_{\mu}$ selected sample which doesn't include appeared $\nu_{e}$ 's. The effect of large genie parameters on the both $\nu_{e}$ and $\nu_{\mu}$ selected spectra is shown in Table 5.3 and $5.4 \ldots \ldots$. . . . . . . . . . . . . . 93

5.17 Shows the effect of GENIE parameter DISvnCC2pi (left) and DISvpNC2pi (right) on $\nu_{\mu}$ selected sample which doesn't include appeared $\nu_{e}$ 's. The effect of large genie parameters on the both $\nu_{e}$ and $\nu_{\mu}$ selected spectra is

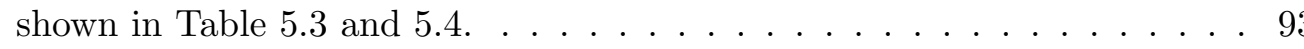

5.18 Shows the effect of GENIE parameter DISvpNC3pi (left) and DISvnNC1pi (right) on $\nu_{\mu}$ selected sample which doesn't include appeared $\nu_{e}$ 's. The effect of large genie parameters on the both $\nu_{e}$ and $\nu_{\mu}$ selected spectra is

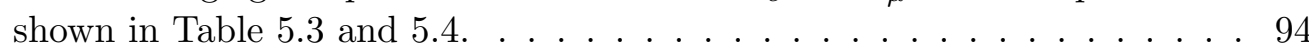

5.19 Shows the effect of GENIE parameter DISvnNC2pi (left) and FormZone (right) on $\nu_{\mu}$ selected sample which doesn't include appeared $\nu_{e}$ 's. The effect of large genie parameters on the both $\nu_{e}$ and $\nu_{\mu}$ selected spectra is shown in Table 5.3 and $5.4 \ldots$. . . . . . . . . . . . . . . . 94

5.20 Shows the effect of GENIE parameter MaCCRES (left) and MvCCRES (right) on $\nu_{\mu}$ selected sample which doesn't include appeared $\nu_{e}$ 's. The effect of large genie parameters on the both $\nu_{e}$ and $\nu_{\mu}$ selected spectra is

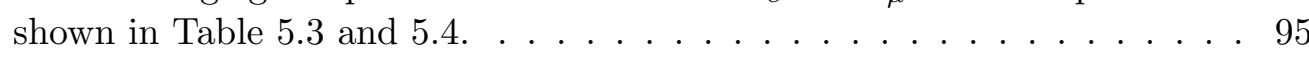

5.21 Shows the effect of GENIE parameter MaNCRES (left) and MFP_pi (right) on $\nu_{\mu}$ selected sample which doesn't include appeared $\nu_{e}$ 's. The effect of large genie parameters on the both $\nu_{e}$ and $\nu_{\mu}$ selected spectra is shown in Table 5.3 and $5.4 \ldots \ldots$. . . . . . . . . . . . . 95

5.22 Shows the effect of GENIE parameter MFP_N (left) and FrAbs_pi (right) on $\nu_{\mu}$ selected sample which doesn't include appeared $\nu_{e}$ 's. The effect of large genie parameters on the both $\nu_{e}$ and $\nu_{\mu}$ selected spectra is shown in Table 5.3 and $5.4 \ldots \ldots \ldots \ldots$. . . . . . . . . . . . . . . . . . . . .

5.23 Effect of calibration offset shown for both $\nu_{e}$ (left) and $\nu_{\mu}$ (Right). Effect is large below $1 \mathrm{GeV}$ for $\nu_{\mu}$ 's. . . . . . . . . . . . . . . 996

5.24 Effect of calibration shape shown for both $\nu_{e}$ (left) and $\nu_{\mu}$ (Right). Effect is large below $1 \mathrm{GeV}$ for $\nu_{\mu}$ 's. . . . . . . . . . . . . . . . 96

5.25 Effect of calibration offset is shown on $\theta_{\mu e}$ limits. . . . . . . . . . . . . 97

5.26 Effect of calibration shape is shown on $\theta_{\mu e}$ limits. . . . . . . . . . . . . 97

5.27 Effect of calibration +ve offset shown for $\nu_{e}$ selected TotalMC (left) and $\nu_{\mu}$ (Right). . . . . . . . . . . . . . . . . 98

5.28 Effect of calibration + ve offset shown for $\nu_{e}$ selected beam $\nu_{e}$ (left) and NC (Right). . . . . . . . . . . . . . . . . . . . . 98

5.29 Effect of calibration -ve offset shown for $\nu_{e}$ selected TotalMC (left) and $\nu_{\mu}$ (Right). . . . . . . . . . . . . . . . 99

5.30 Effect of calibration -ve offset shown for $\nu_{e}$ selected beam $\nu_{e}$ (left) and NC (Right). . . . . . . . . . . . . . . . . . . . . . . 99

5.31 Effect of calibration shape shown for $\nu_{e}$ selected TotalMC (left) and $\nu_{\mu}$ (Right). . . . . . . . . . . . . . . . . . 100

5.32 Effect of calibration shape shown for $\nu_{e}$ selected beam $\nu_{e}$ (left) and $\mathrm{NC}$ (Right). . . . . . . . . . . . . . . . . . 100

5.33 Effect of calibration + ve offset shown for $\nu_{\mu}$ selected TotalMC (left) and $\nu_{\mu}$ (Right). . . . . . . . . . . . . . . . 101 
5.34 Effect of calibration +ve offset shown for $\nu_{\mu}$ selected $\nu_{e}$ (left) and $\nu_{\mu}$ (Right) . . . . . . . . . . . . . . . . . . . . 101

5.35 Effect of calibration offset shown for both $\nu_{\mu}$ selected TotalMC (left) and $\nu_{\mu}$ (Right). . . . . . . . . . . . . . . . . 102

5.36 Effect of calibration -ve offset shown for $\nu_{\mu}$ selected beam $\nu_{e}$ (left) and NC (Right). . . . . . . . . . . . . . . . . . . 102

5.37 Effect of calibration shape shown for $\nu_{\mu}$ selected TotalMC (left) and $\nu_{\mu}$ (Right). . . . . . . . . . . . . . . . . . . . . 103

5.38 Effect of calibration offset shown for $\nu_{\mu}$ selected beam $\nu_{e}$ (left) and NC (Right). . . . . . . . . . . . . . . . . . . . 103

5.39 Showing the effect of rocks for both $\nu_{e}$ (left) and $\nu_{\mu}$ (right) selected sample. Effect is large below $1 \mathrm{GeV}$ for $\nu_{\mu}$ 's. . . . . . . . . . . . . . . . 104

5.40 Effect of light level is shown for both $\nu_{e}$ (left) and $\nu_{\mu}$ (right). Effect is large below $1 \mathrm{GeV}$ for $\nu_{\mu}$ 's. . . . . . . . . . . . . . . . . 104

5.41 Effect of light level systematics on the sensitivity. . . . . . . . . . . . 105

5.42 Effect of cherenkov is shown for both $\nu_{e}$ (left) and $\nu_{\mu}$ (right). Effect is large below $1 \mathrm{GeV}$ for $\nu_{\mu}$ 's. . . . . . . . . . . . . . . 105

5.43 Effect of Cherenkov systematics on $\theta_{\mu e}$ limits is shown. . . . . . . . . . 106

5.44 The distribution of true energy for flux swap $\nu_{e}$ 's and beam $\nu_{\mu}$ 's with no selection applied (left). We also applied the PPFX and cross-section weights. Right plot shows the ratio of $\nu_{e}$ and $\nu_{\mu}$ cross-sections. . . . . . 106

5.45 The distribution of true length over true energy for the $\nu_{e}$ selected appeared $\nu_{e}$ 's and $\nu_{\mu}$ selected beam $\nu_{\mu}$ 's. The orange histogram is a projection onto $\mathrm{L} / \mathrm{E}$ axis from the $2 \mathrm{D}$ spectrum in extrapolation object. . . . . . 107

5.46 The double ratio of true / extrap as true L/E for both appeared $\nu_{e}$ and $\nu_{\mu}$ 's. The double ratio is flat across true L/E. . . . . . . . . . . . . 107

5.47 Showing the method followed to get an estimate of the acceptance effect using the $\nu_{e}$ and $\nu_{\mu}$ selected sample. . . . . . . . . . . . . 109

5.48 Show the ratio of efficiencies for both beam and appeared $\nu_{e}$ 's in true energy. . . . . . . . . . . . . . . . . . 110

5.49 Showing the distribution of reconstructed energy after the $\nu_{e}$ (left) and $\nu_{\mu}$ (right) selection applied with and without cosmic noise. We use CVN selector for the selection of both $\nu_{e}$ and $\nu_{\mu} \ldots \ldots \ldots \ldots \ldots$

5.50 Shows the distribution of spill POT for both data and MC. . . . . . . . 112

5.51 Shows the number of slices per spill POT as a function of spill POT with no selection applied (left) and CVNe $>0.95$ applied to select the $\nu_{e}$ 's. . . 113

6.1 The 3+1 model probabilities for both $\nu_{e}$ appearance (left), $\nu_{\mu}$ disappearance (middle) and $\nu_{e}$ disappearance (right) at the LSND best fit point $\left(\sin ^{2} 2 \theta, \Delta \mathrm{m}^{2}\right)=\left(0.003,1.2 \mathrm{eV}^{2}\right)$ and the global best-fit $\left(\sin ^{2} 2 \theta, \Delta \mathrm{m}^{2}\right)=$ $\left(0.00048,1.7 \mathrm{eV}^{2}\right)[87,88]$. The probability at the LSND best-fit is shown in black and the probability for the global best-fit is shown in red. To use the LSND limits which are provided in $\theta_{\mu e}$, we set $\theta_{14}$ to 10 degrees driven by the Bugey limits on this angle. . . . . . . . . . . . 116

6.2 True L/E for all beam $\nu_{e}$ 's (left) and $\nu_{\mu}^{\prime} s$ (right) at the NOvA near detector.116

6.3 The number of appeared $\nu_{e}$ 's at LSND best-fit point as a function of true $\mathrm{L} / \mathrm{E}$ with no selection (left) and with $\nu_{e}$ selection applied (right) at the NOvA Near Detector. . . . . . . . . . . . . . . . . . . . . . 117

6.4 True L for all beam $\nu_{e}$ 's (left) and $\nu_{\mu}$ 's (right) at the NOvA near detector. 117 
6.5 True E for all beam $\nu_{e}$ 's (left) and $\nu_{\mu}$ 's (right) at the NOvA near detector. 117

6.6 Showing the distribution of reconstructed energy after the $\nu_{e}$ (top) and $\nu_{\mu}$ (bottom) selection applied. The dotted line indicates the total prediction in a $3+1$ model using the oscillation parameters shown on the plot. To use the LSND limits which are provided in $\theta_{\mu e}$, we set $\theta_{14}$ to 10 degrees driven by the Bugey limits on this angle. The solid lines show the 3 -flavor prediction, the dotted at the 2017 Global fit best fit, and the dashed at the LSND best fit. . . . . . . . . . . . . . . . . . . . 125

6.7 Comparison of $\sin ^{2} \theta_{14}$ Vs $\Delta m_{41}^{2}$ sensitivity between single fit (red) and joint fit (black) with systematics (dashed) and without systematics (solid).127

6.8 Showing the limits for $\theta_{14}$ in $\sin ^{2} 2 \theta_{14}$ Vs $\Delta m_{41}^{2}$ surface, while profiling over $\theta_{24}$ We also compare the $\theta_{14}$ limits with Bugey results at $90 \%$ C.L. . 129

6.9 Sensitivity for $\theta_{24}$ while profiling over $\theta_{34}$ and $\theta_{14}$ at $90 \%$ C.L. The regions excluded by CDHS, CCFR, MiniBooNE/SciBooNE are also shown. . . . . 130

6.10 Sensitivity for $\theta_{\mu e}$ while profiling over $\theta_{34}$ at $90 \%$ C.L. for $8.05 \mathrm{e}+20$ POT. The regions excluded by LSND, MiniBooNE and KARMEN are also shown.131

6.11 The distribution of reconstructed energy of $\nu_{e}$ selected events (left) and $\nu_{\mu}$ selected events with CVN (right) with the Poisson fluctuations applied in every energy bin. . . . . . . . . . . . . . . . . . 131

6.12 Left: The sensitivities after fitting with fake data (black) at 90\% C.L., with mock data (solid red) at $90 \%$ C.L. These sensitivities are made for 8.05e+20 POT. Right: 200 mock data universes with only statistical fluctuations. . . . . . . . . . . . . . . . . . . . 132

6.13 Show the reconstructed energy distribution of nominal unoscillated prediction and the expected oscillated prediction at LSND best fit mixing parameters for both $\nu_{e}$ (left) and $\nu_{\mu}$ selected sample. . . . . . . . . . 132

6.14 The sensitivities after fitting with LSND best fit signal injection with no systematics (red) at 90\% C.L., with all systematics (black) at 90\% C.L. These sensitivities are made for 8.05e+20 POT. . . . . . . . . . . . 133

6.15 Shows the preselection distribution of reconstructed neutrino energy for the events which pass the $\nu_{e}$ data quality (left) and fiducial (right) selection. The MC is normalized to the data POT of $8.05 \mathrm{e}+20 \ldots \ldots . . .133$

6.16 Shows the preselection distribution of reconstructed neutrino energy for the events which pass the $\nu_{e}$ containment (left) and front planes (right) selection. The MC is normalized to the data POT of $8.05 \mathrm{e}+20$. . . . . 134

6.17 Shows the preselection distribution of reconstructed neutrino energy for the events which pass the $\nu_{e}$ number of hits (left) and energy (right) selection. The MC is normalized to the data POT of $8.05 \mathrm{e}+20$. . . . . . 134

6.18 Shows the preselection distribution of reconstructed neutrino energy for the events which pass the $\nu_{e}$ prong length (left) and Ptp (right) selection. The MC is normalized to the data POT of $8.05 \mathrm{e}+20 \ldots \ldots . \ldots 135$

6.19 Shows the preselection distribution of reconstructed neutrino energy for the events which pass the $\nu_{\mu}$ quality (left) and containment (right) selection. The MC is normalized to the data POT of $8.05 \mathrm{e}+20 \ldots . . .2 .135$

6.20 Shows the distribution of the number of vertices (left) and vertex X (right) with the $\nu_{e}$ selection applied. . . . . . . . . . . . . 136

6.21 Shows the distribution of the vertex $\mathrm{Y}$ (left) and vertex $\mathrm{Z}$ (right) with the $\nu_{e}$ selection applied. . . . . . . . . . . 136 
6.22 Shows the distribution of the number of showers (left) and shower start X (right) with the $\nu_{e}$ selection applied. . . . . . . . . . . . . 137

6.23 Shows the distribution of the shower start $\mathrm{Y}$ (left) and shower start Z (right) with the $\nu_{e}$ selection applied. . . . . . . . . . . . 137

6.24 Shows the distribution of the shower stop X (left) and shower stop Y (right) with the $\nu_{e}$ selection applied. . . . . . . . . . . 138

6.25 Shows the distribution of the shower stop Z (left) with the $\nu_{e}$ selection applied. . . . . . . . . . . . . . . . . . 138

6.26 Shows the distribution of the number of cells from edge (left) and the number of tracks (right) with the $\nu_{\mu}$ selection applied. . . . . . . . . 139

6.27 Shows the distribution of track start X (left) and track start Y (right) with the $\nu_{\mu}$ selection applied.. . . . . . . . . . . . . . . . 139

6.28 Shows the distribution of track start Z (left) and track stop X (right) with

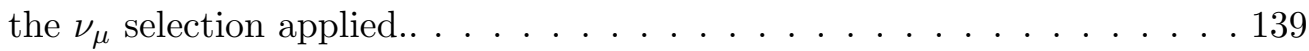

6.29 Shows the distribution of track stop Y (left) and track stop Z (right) with the $\nu_{\mu}$ selection applied.. . . . . . . . . . . . . . 140

6.30 Shows the distribution of CVN for the $\nu_{\mu}$ selected events after the CVNm

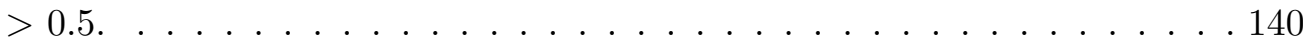

6.31 Shows the distribution of CVNe for the $\nu_{e}$ preselected sample (left) and the distribution of reconstructed neutrino energy for the $\nu_{e}$ selected sample with $\mathrm{CVNe}>0.95$ (right). $\mathrm{MC}$ is normalized to the data POT of

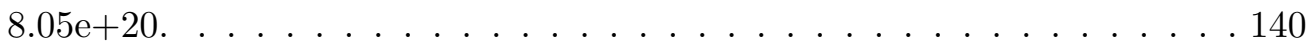

6.32 Shows the distribution of reconstructed neutrino energy for the $\nu_{e}$ preselected sample (left) and the fully selected sample with $\mathrm{CVNe}>0.95$ (right). MC is normalized to the data POT of $8.05 \mathrm{e}+20 \ldots . . . . . .141$

C.1 Altra figura experimental . . . . . . . . . . . . . . . . . . 157

C.2 Shows the effect of GENIE parameter FrPiProd_N on both $\nu_{e}$ (left) and $\nu_{\mu}$ selected sample which doesn't include appeared $\nu_{e}$ 's. . . . . . . . . 157

C.3 Shows the effect of GENIE parameter CCQEPauliSupViaKF on both $\nu_{e}$ (left) and $\nu_{\mu}$ selected sample which doesn't include appeared $\nu_{e}$ 's. . . . . 158

C.4 Shows the effect of GENIE parameter CCQEMomDistroFGtoSF on both $\nu_{e}$ (left) and $\nu_{\mu}$ selected sample which doesn't include appeared $\nu_{e}$ 's. . . . 159

C.5 Shows the effect of GENIE parameter RDecBR1gamma on both $\nu_{e}$ (left) and $\nu_{\mu}$ selected sample which doesn't include appeared $\nu_{e}$ 's. . . . . . . . 159

C.6 Shows the effect of GENIE parameter RDecBR1eta on both $\nu_{e}$ (left) and $\nu_{\mu}$ selected sample which doesn't include appeared $\nu_{e}$ 's. . . . . . . . 160

C.7 Shows the effect of GENIE parameter Theta_Delta2Npi on both $\nu_{e}$ (left) and $\nu_{\mu}$ selected sample which doesn't include appeared $\nu_{e}^{\prime}$ 's. . . . . . 160 


\section{List of Tables}

1.1 Experimental determination of oscillation parameters by various type of neutrino experiments $[71] \ldots \ldots \ldots \ldots 14$

2.1 Most common decay modes and the branching fractions of $\pi \mathrm{s}, \mathrm{K}$ 's and $\mu$ 's to neutrinos. . . . . . . . . . . . . . . . . 35

2.2 The composition of the liquid scintillator used in NOvA. This table is taken NOvA technical design report. . . . . . . . . . . . . 40

5.1 Summary of all $\nu_{e}$ systematics. . . . . . . . . . . . . . . . 80

5.2 Summary of all $\nu_{\mu}$ systematics. . . . . . . . . . . . . . . . . . 80

5.3 Systematic summary of $\nu_{e}$ sample. The top six are large GENIE parameters included as individual pulls. All other GENIE parameters are included in the summed small genie. All five pc for PPFX are shown. . . 80

5.4 Systematic summary of $\nu_{\mu}$ sample. The $\pm 1 \sigma$ shifts are the ratio between the $1 \sigma$ shifted and nominal un-oscillated prediction. The top seven are large GENIE parameters included as individual pulls, all other GENIE parameters included in the summed small genie. All five principal components for PPFX are shown. . . . . . . . . . . . . . . 81

5.5 Shows the $\pm 1 \sigma \%$ shifts for the large GENIE systematic parameters having more $1 \%$ effect on the $\nu_{e}$ selected sample. Only the parameters having larger than $2 \%$ effect are included in the fit. . . . . . . . . . . . . 85

5.6 Shows the $\pm 1 \sigma \%$ shifts for the large GENIE systematic parameters having more than $1 \%$ effect on the $\nu_{\mu}$ selected sample. . . . . . . . . . . 86

5.7 Effect of NC GENIE parameters on the $\nu_{e}$ selected sample. . . . . . . . . . 88

5.8 Effect of NC GENIE parameters on the $\nu_{\mu}$ selected sample. . . . . . . . 89

5.9 GENIE NC systematic parameters. . . . . . . . . . . . . . . . . . . 89

6.1 Table shows the cut flow for the selected events using non swap sample using the $\nu_{e}$ selection with no oscillation. Here, efficiency $=\nu_{e}$ 's selected with full selection $/ \nu_{e}$ 's having true vertex in the fiducial volume. These numbers are corresponding to a data POT 8.05e+20 . . . . . . . . . . 124

6.2 Table shows the cut flow for the selected events using non swap sample using the $\nu_{\mu}$ selection with no oscillation. Here, efficiency is defined as $\nu_{\mu}$ 's selected with full selection $/ \nu_{\mu}$ 's which are contained. These numbers are corresponding to a data POT $8.05 \mathrm{e}+20$. . . . . . . . . . . . . . 124

6.3 This table shows the event rates expected for $\nu_{e}$ appearance channel at the LSND best fit point $\left(\sin ^{2} 2 \theta, \Delta \mathrm{m}^{2}\right)=\left(0.003,1.2 \mathrm{eV}^{2}\right)$ and the global best-fit $\left(\sin ^{2} 2 \theta, \Delta \mathrm{m}^{2}\right)=\left(0.00048,1.7 \mathrm{eV}^{2}\right)[87,88]$ oscillation parameters. 125 
6.4 This table shows the event rates expected for $\nu_{\mu}$ appearance channel at the LSND best fit point $\left(\sin ^{2} 2 \theta, \Delta \mathrm{m}^{2}\right)=\left(0.003,1.2 \mathrm{eV}^{2}\right)$ and the global best-fit $\left(\sin ^{2} 2 \theta, \Delta \mathrm{m}^{2}\right)=\left(0.00048,1.7 \mathrm{eV}^{2}\right)[87,88]$ oscillation parameters. 126

C.1 Shows the $\pm 1 \sigma \%$ shifts for all the GENIE systematic parameters for the $\nu_{e}$ selected sample. Continued in the next page. . . . . . . . . . . . 152

C.2 Shows the $\pm 1 \sigma \%$ shifts for all the GENIE systematic parameters for the $\nu_{e}$ selected sample. . . . . . . . . . . . . . . . . 153

C.3 Shows the $\pm 1 \sigma \%$ shifts for all the GENIE systematic parameters for the $\nu_{\mu}$ selected sample. Continued in the next page. . . . . . . . . . 155

C.4 Shows the $\pm 1 \sigma \%$ shifts for the remaining GENIE systematic parameters for the $\nu_{\mu}$ selected sample. . . . . . . . . . . . . . . . . 156 
DEDICATED TO MY ENTIRE FAMILY 



\section{Chapter 1}

\section{Introduction to Neutrino Physics}

\subsection{The Beginning}

Radioactivity was first discovered by Henri Becquerel in Uranium in 1896 [1-4], and, two years later, by Madame Curie in Thorium [5]. This radioactivity was categorized into two types by Rutherford in 1911, $\alpha$ and $\beta$ decay, where the decay products, namely $\alpha$ (Helium nuclei) and $\beta$ (electrons) particles were assumed to be ejected at fixed energies from the nuclei. This was in contradiction with what James Chadwick has observed in 1914 [6], where he saw a continuous spectrum for the emitted $\beta$ particles. This surprising result was confirmed in 1927 by Ellis and Wooster [7]. This was a serious problem because this result questioned the fundamental conservation laws. Many theories were proposed to explain the missing energy, but nothing succeeded.

In 1930, Wolfgang Pauli [8] came up with a brilliant explanation that there must be a neutral weakly interacting particle emitted along with the other particles, which was taking away the missing energy in $\beta$-decay. He called this particle "neutron" which is a charge-less particle spin $1 / 2$. But, after the discovery of the neutral particle by James Chadwick [6] which also got called the neutron, Enrico Fermi renamed it as "neutrino", in Italian which means small and neutral. It was concluded that neutrinos were massless, neutral and weakly interacting fermions. Even with such elusive behavior, they had the potential to provide the answers to some fundamental questions in physics. 


\subsection{How can a Neutrino Interact?}

In 1934, Enrico Fermi formulated the theory of $\beta$-decay from which the theory of weak interactions originated [9]. He made it analogous to the theory of Quantum Electrodynamics (QED). Fermi's theory is a 4-point interaction theory, but this allows parity to be violated. In 1936, George Gamow and Edward Teller [10] introduced axial-vector currents to retain parity conservation. Jabez Curry Street and E. C. Stevenson observed the decay of muon $(\mu)$ [11] which further strengthened Fermi's weak interaction theory in 1937. Although, not a single neutrino interaction is found.

The Lagrangian for $\beta$-decay as written by Fermi involving only vector currents (in analogy with QED), is written as,

$$
L_{\beta}=G_{F} j_{\mu}^{n \rightarrow p} j_{\nu \rightarrow e}^{\mu}=G_{F}\left(\bar{u}_{p} \gamma_{\mu} u_{n}\right)\left(\bar{u}_{e} \gamma^{\mu} u_{\nu}\right)
$$

where $G_{F}$ is known as the Fermi coupling constant, this we now what we call the weak force. $j_{\mu}^{n \rightarrow p}$ and $j_{\nu \rightarrow e}^{\mu}$ are the hadronic and leptonic currents respectively. There were many shreds of evidence for the parity violation in weak interactions such as $\theta$ - $\tau$ puzzle where the same particle, $\mathrm{K}^{+}$observed to decay in two different modes. In 1956, Tsung-Dao Lee and Chen-Ning Yang took charge on explaining this problem theoretically to convey that parity is violated in weak interactions, specifically in $\beta$ decay. They also proposed an experiment to test their hypothesis experimentally [12] and worked with Chien-Shiung $\mathrm{Wu}$ to test this. Wu's experimental results [13] were astounding and confirmed that parity is violated in weak interactions. Based on these results, the Lagrangian had to be modified to incorporate parity violation. After Wu's results, Lee and Yang postulated that all neutrinos are left-handed and antineutrinos are right-handed. The Hamiltonian after adding the parity violation term is

$$
H_{w}=\frac{G_{F}}{\sqrt{2}}\left[\bar{u}_{p} \gamma_{\mu}\left(1-g_{A} \gamma_{5}\right) u_{n}\right]\left[\bar{u}_{e} \gamma^{\mu}\left(1-\gamma_{5}\right) u_{\nu}\right]+h . c
$$

This Hamiltonian has the Vector-Axial current structure involving parity violation, was proposed by Ennackal Chandy George Sudarshan and Robert Eugene Marshak in 1957 [14] and by Richard Phillips Feynman and Murray Gell-Mann in 1958 [15]. The VA theory was very successful and has been used to successfully explain all the charged current weak currents successfully, for example, it can explain the weak decay of particles like muons, pions, kaon, hyperons. It is an effective theory. 


\subsection{Neutrinos in Standard Model}

Standard Model (SM) is a gauge theory describes all three fundamental interactions namely the strong, electromagnetic and weak interactions of elementary particles in nature. $\mathrm{SM}$ is based on the local symmetry group, $\mathrm{SU}(3)_{C} \times \mathrm{SU}(2)_{L} \times \mathrm{U}(1)_{Y}$. There are eight massless gluons which mediate strong interactions, four gauge bosons $\left(\mathrm{W}^{ \pm}, \mathrm{Z}\right.$, and $\gamma$ photon) which mediate weak and electromagnetic interactions. These all correspond to the generators of the $\mathrm{SU}(3), \mathrm{SU}(2)$ and $\mathrm{U}(1)$ group. The symmetry gauge group $\mathrm{SU}(2)_{L} \times$ $\mathrm{U}(1)_{Y}$ describes the interactions involving neutrinos. The generation of masses for the fundamental particles is attained by incorporating the Higgs mechanism in the SM. The fermion mass is generated by the Higgs mechanism through the Yukawa coupling of fermion fields with the Higgs.

SM has three generations of quarks and leptons, and each of them is an isospin doublet as shown in Eq. 1.3. In the case of leptons, every neutrino flavour is associated with its corresponding lepton flavour. All the fermions before symmetry breaking are massless, neutrino too. But after the symmetry breaking, all the leptons attain mass due to Yukawa coupling with Higgs field, where as neutrinos remain massless because of the lack of right-handed neutrinos.

$$
\begin{gathered}
\left(\begin{array}{l}
u \\
d
\end{array}\right),\left(\begin{array}{l}
c \\
s
\end{array}\right) \text { and }\left(\begin{array}{l}
t \\
b
\end{array}\right) \quad \text { Quarks } \\
\left(\begin{array}{c}
\nu_{e} \\
e
\end{array}\right),\left(\begin{array}{c}
\nu_{\mu} \\
\mu
\end{array}\right) \text { and }\left(\begin{array}{c}
\nu_{\tau} \\
\tau
\end{array}\right) \quad \text { Leptons }
\end{gathered}
$$

Neutrinos interact with the matter with the exchange of weak gauge bosons, $\mathrm{W}^{ \pm}$and $\mathrm{Z}^{0}$. Charged current interactions mediated by a $\mathrm{W}$ boson give a charged lepton of same neutrino flavour along with a hadron as shown in Fig. 1.1. Neutral current interactions mediated by a Z boson don't produce any charged leptons which make the $\mathrm{NC}$ interactions indistinguishable as shown in Fig. 1.1.

\subsection{Neutrino Mass}

Neutrinos are massless in SM. With the electroweak symmetry breaking, the Higgs mechanism, mass is attained by the particles via a Yukawa coupling with the Higgs field, but 


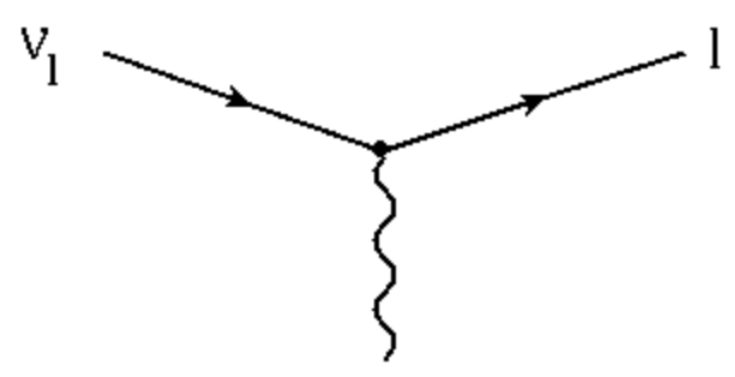

W

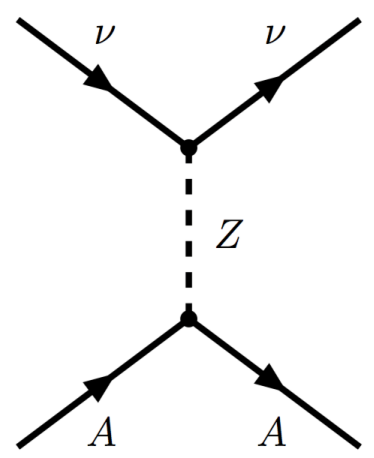

Figure 1.1: The Feynman diagrams for the weak neutrino charged current (left) and neutral current (right).

neutrinos remain massless because of the lack of right-handed neutrinos. Experimental evidence from neutrino oscillation experiments, proves that they do have has mass and so requires a new physics theory (beyond Standard Model) to explain it. One such theory is a See-Saw mechanism.

Two possible mass terms can be added; a Dirac mass term

$$
m_{D} \bar{\psi} \psi=m_{D} \bar{\psi}_{L} \psi_{R}+m_{D} \bar{\psi}_{R} \psi_{L}
$$

and a Majorana mass term can be obtained just by replacing $\psi_{R}$ with $\psi_{L}^{C}=C \bar{\psi}_{L}^{T}$

$$
m_{L} \bar{\psi}^{C}{ }_{L} \psi_{L}+m_{R} \overline{\psi_{R}^{C}} \psi_{R}
$$

Then, the total Lagrangian is written as follows

$$
\begin{aligned}
L_{m} & =\frac{1}{2}\left(L_{L}^{D}+L_{R}^{D}+L_{L}^{M}+L_{R}^{M}\right)+h . c \\
& =m_{D} \bar{\psi}_{R} \psi_{L}+m_{D} \overline{\psi^{C}}{ }_{L} \psi_{R}^{C}+m_{L} \bar{\psi}^{C}{ }_{L} \psi_{L}+m_{R} \bar{\psi}_{R}^{C} \psi_{R}
\end{aligned}
$$

This can be written in a matrix form with $m=\left(\begin{array}{ll}m_{L} & m_{D} \\ m_{D} & m_{R}\end{array}\right)$ as

$$
L_{m} \approx\left(\begin{array}{cc}
\bar{\psi}_{L}^{C} & \bar{\psi}_{R}
\end{array}\right)\left(\begin{array}{ll}
m_{L} & m_{D} \\
m_{D} & m_{R}
\end{array}\right)\left(\begin{array}{c}
\psi_{L} \\
\psi_{R}^{C}
\end{array}\right)
$$

If we diagonalize the matrix, then the mass of mass eigen states can be expressed in terms of $\mathrm{m}_{D}, \mathrm{~m}_{L}$ and $\mathrm{m}_{R}$ as 


$$
m_{1,2}=\frac{1}{2}\left[\left(m_{L}+m_{R}\right) \pm \sqrt{\left(m_{L}-m_{R}\right)^{2}+4 m_{D}^{2}}\right]
$$

If we assume $m_{L}=0$ and $m_{R}>>m_{D}$, then the Eq. 1.10 becomes

$$
\begin{aligned}
& m_{1}=\frac{m_{D}^{2}}{m_{R}} \quad \text { mass of the field } \nu_{1} \\
& m_{2}=m_{R}\left(1+\frac{m_{D}^{2}}{m_{R}^{2}}\right) \approx m_{R} \quad \text { mass of the field } \nu_{2}
\end{aligned}
$$

As we see from Eq. 1.11, if the mass of one neutrino is large, then the other mass of the neutrino is very small because of the presence of suppression factor $\frac{1}{m_{R}}$. This mechanism naturally explains the relatively smallness of the neutrino mass.

\subsection{Three Generations of Neutrinos}

Neutrinos are very hard to detect because of their extremely weak interaction crosssection with the Standard Model matter, but it is not impossible. In 1956, Frederick Reines with Clyde Lorrain Cowan observed the first ever neutrino (anti-electron neutrino) interaction in their cleverly designed reactor experiment [16] to test the inverse $\beta$-decay in which an anti-neutrino can produce a positron and neutron, $\overline{\nu_{e}}+\mathrm{p} \rightarrow \mathrm{n}+\mathrm{e}^{+}$. The detection technology Reines and Cowan used to detect the products from the inverse $\beta$-decay deployed a liquid scintillation counter. It was a much-awaited for discovery in physics history. They were awarded the Nobel Prize in 1995 for their ground breaking discovery. Raymond Davis Jr discovered the electron neutrinos from the sun in 1968.

In 1962, Leon Lederman, Melvin Schwartz, Jack Steinberger et al. [17], conducted an experiment to discover the second type of neutrino, called a muon neutrino at Brookhaven National Laboratory. This is the first accelerator experiment which used neutrinos coming from the pion decay. To the world's surprise, they discovered the muon neutrino which established the second generation of leptons. They awarded the Nobel Prize in 1988 for their discovery.

In 1975, the tau lepton was discovered which hinted the existence of tau neutrino. In 2000, the third type of neutrino, called tau neutrino, was discovered by the DONUT experiment at Fermilab. Tau neutrino produces a tau lepton when it interacts with 
atomic nuclei, and the tau lepton and its resulting decay products were recorded with the emulsion target and detected by scintillators.

\subsection{Common Source of Neutrinos}

There are many sources of neutrinos. In the fusion reactions, as shown in Fig. 1.2, happening inside the star in proton-proton and $\mathrm{C}-\mathrm{N}$ chain reactions at energies in the $O(\mathrm{MeV})$ emit neutrinos.

The atmosphere is another major source of neutrinos. When a cosmic ray (for example a proton and Helium) with high energies hits a molecule in the upper atmosphere, a shower of particles emitted (including pions and kaons). These decay further to give neutrinos at energies in the $O(\mathrm{GeV})$ as shown in Fig. 1.3.

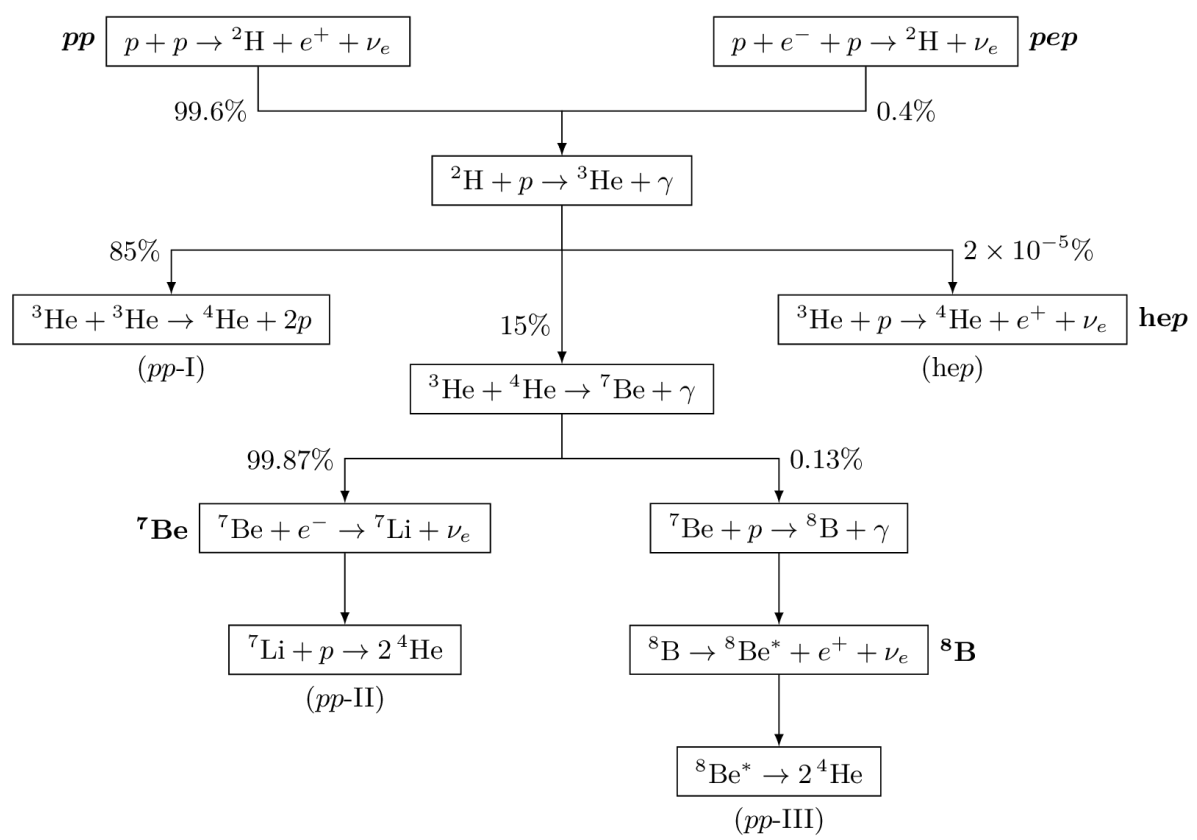

Figure 1.2: Shows the various processes involved the p-p cycle inside a star. Neutrinos emitted almost in every reaction to carry away some of the energy. This figure is taken from Carlo Giunti and Chung W. Kim [18].

Nuclear reactors are another major source of neutrinos, here neutrinos produced in the nuclear fission reactions in the $O(\mathrm{GeV})$. Reactors are a source of pure anti-electron neutrinos as explained in Fig. 1.4.

Another important source of neutrinos is accelerators. For example, the Main Injector at Fermilab makes a huge amount of neutrinos as shown in Fig. 2.2. Also, there are 


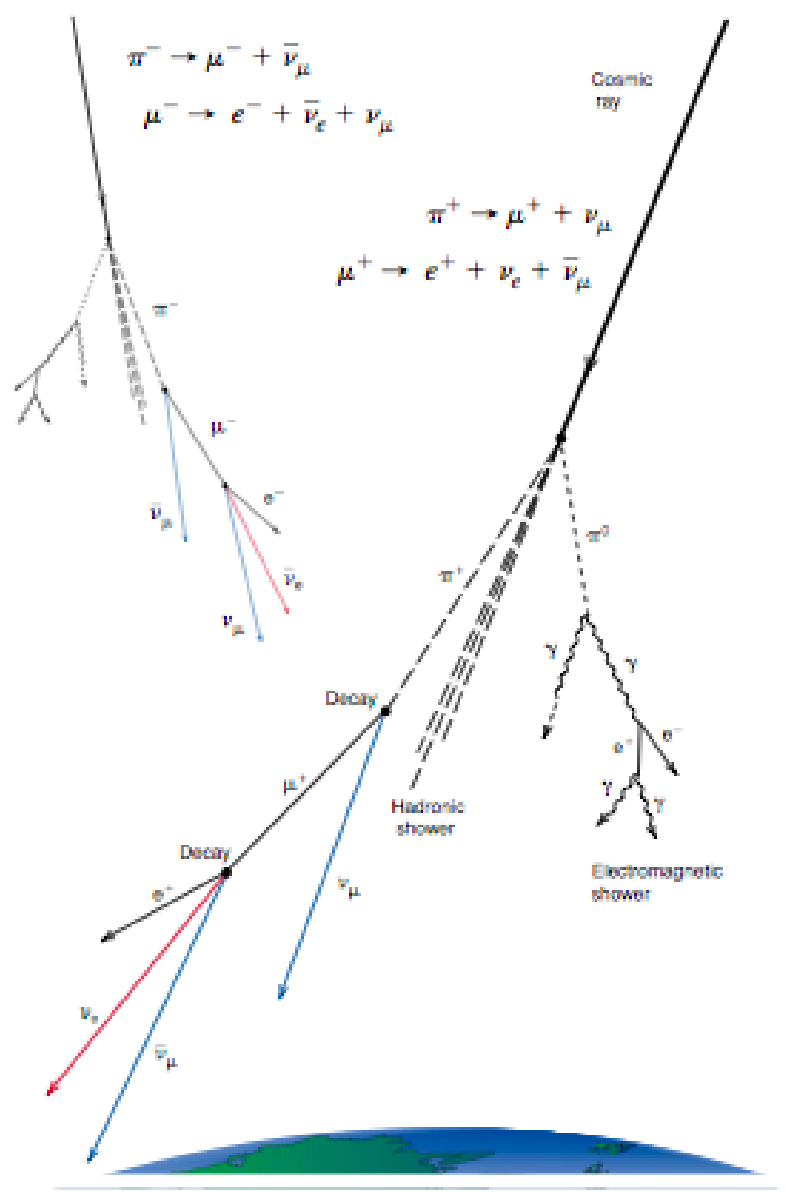

FiguRE 1.3: Shows the various processes involved in the generation of neutrinos in the upper atmosphere. This process happens at an altitude of $\approx 30 \mathrm{~km}$. Figure taken from Los Alamos Science 25 (1997) [19].

other sources of neutrinos like an accretion of matter into black holes, supernovae, and gamma-ray bursts.

\subsection{Neutrinos Oscillations}

Neutrinos were considered to be massless until 1998 when Raymond Davis Jr. and John Bahcall published their experimental results measuring the solar neutrino flux emanating from the Sun's Core. They detected only about one-third of as many as they predicted. This is known as "Solar Neutrino Problem". In 1967, B. Pontecorvo [21] predicted this even before Davis first experimental result was [22] published. The Kamiokande experiment $[23,24]$ also observed the same deficiency in the solar neutrino flux. In 2001, Sudbury Neutrino Observatory (SNO) published experimental results which proved that neutrinos are not missing; instead, they are changing into muon and tau neutrinos. The 

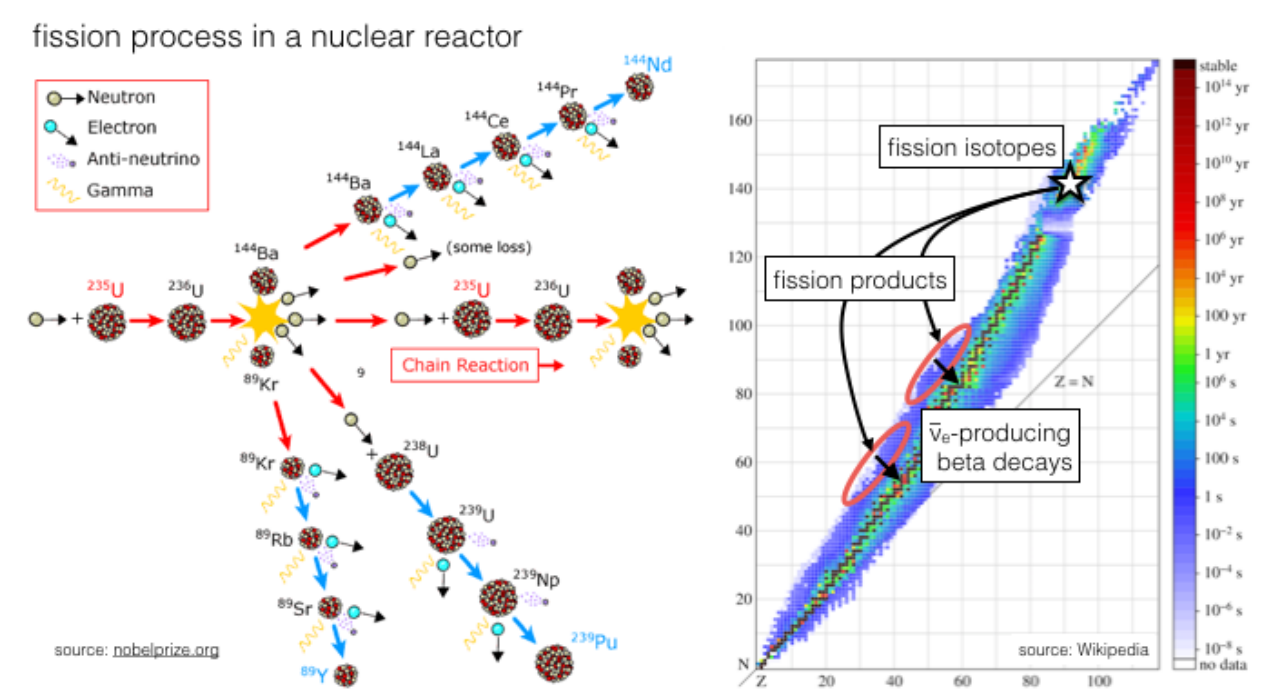

Figure 1.4: Left: Shows the nuclear fission reaction chain. Right: To attain the stability, nuclei undergo several decays emitting electons and anti-electron neutrinos [20].

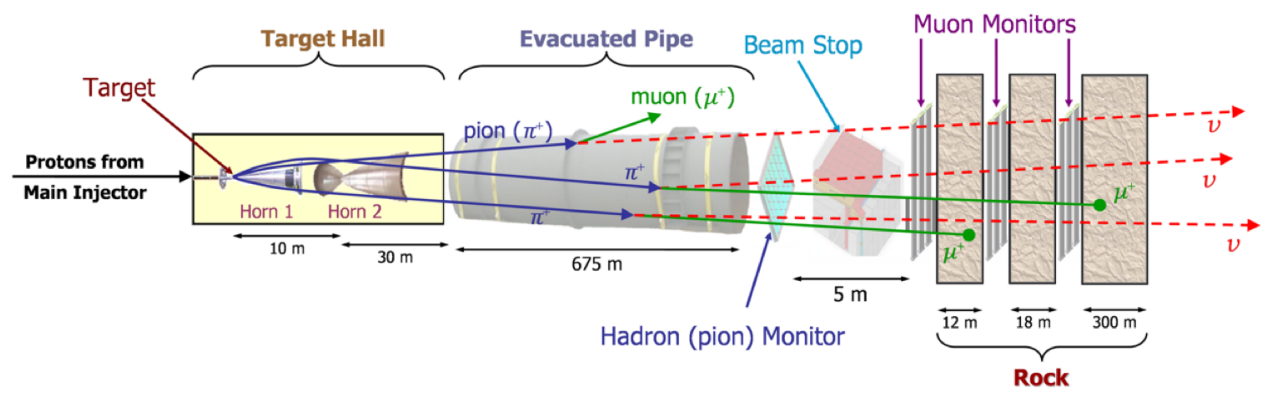

FIgURE 1.5: Shows the generation of neutrino in NuMI facility at Fermilab.

SNO results were confirmed by KamLAND experiment $[25,26]$. The reason why the previous experiments have not seen this is because those experiments were not sensitive to muon and tau neutrinos and could only see electron neutrinos. The property of changing flavour can happen only for particles having non-zero mass. These results are revolutionary and opened the door for another era in the history of physics.

\subsubsection{Neutrino Oscillations in Vaccum}

Neutrino Oscillations is purely a quantum mechanical phenomena proposed by Pontecorvo in 1950s in analogy with $\mathrm{K}^{0}-\bar{K}^{0}$ oscillations. The standard theory of neutrino oscillations in the plane-wave approximation was developed by Eliezer and Swift [27], Fritzsch and Minkowski [28], Bilenky and Pontecorvo [29]. 
Neutrinos are produced via the weak interaction and they interact in a flavor basis $\left|\nu_{e}\right\rangle,\left|\nu_{\mu}\right\rangle,\left|\nu_{\tau}\right\rangle$, but they travel in a mass eigen basis $\left|\nu_{1}\right\rangle,\left|\nu_{2}\right\rangle,\left|\nu_{3}\right\rangle$. Analogous to the Cabbibo-Kobayashi-Maskawa matrix, a group of four people namely, Pontecorvo, Maki, Nakagawa and Sakata [30] developed a mixing formalism for neutrino mixing. In the case of 3-flavour neutrinos, we can write

$$
\begin{gathered}
\left(\begin{array}{l}
\nu_{e} \\
\nu_{\mu} \\
\nu_{\tau}
\end{array}\right)=U\left(\begin{array}{c}
\nu_{1} \\
\nu_{2} \\
\nu_{3}
\end{array}\right), \\
\left(\begin{array}{c}
\nu_{e} \\
\nu_{\mu} \\
\nu_{\tau}
\end{array}\right)=\left(\begin{array}{lll}
\mathrm{U}_{e 1} & \mathrm{U}_{e 2} & \mathrm{U}_{e 3} \\
\mathrm{U}_{\mu 1} & \mathrm{U}_{\mu 2} & \mathrm{U}_{\mu 3} \\
\mathrm{U}_{\tau 1} & \mathrm{U}_{\tau 2} & \mathrm{U}_{\tau 3}
\end{array}\right)\left(\begin{array}{c}
\nu_{1} \\
\nu_{2} \\
\nu_{3}
\end{array}\right)
\end{gathered}
$$

Where $\mathrm{U}$ is the $3 \times 3$ PMNS unitary mixing matrix, $\mathrm{U}^{\dagger} \mathrm{U}=\mathrm{I}$. The PMNS matrix can be expressed in terms of mixing angles $\theta_{i j}$ and $\mathrm{CP}$-violation phase $\delta$ as follows

$$
U=\left(\begin{array}{ccc}
c_{13} c_{12} & c_{13} s_{12} & s_{13} e^{-i \delta} \\
-c_{23} s_{12}-s_{23} c_{12} s_{13} e^{i \delta} & c_{23} c_{12}-s_{23} s_{12} s_{13} e^{i \delta} & c_{13} s_{23} \\
s_{23} s_{12}-c_{23} c_{12} s_{13} e^{i \delta} & -s_{23} c_{12}-c_{23} s_{12} s_{13} e^{i \delta} & c_{13} s_{23}
\end{array}\right)
$$

Where $\mathrm{c}_{i j}=\cos \theta_{i j}$ and $\mathrm{s}_{i j}=\sin \theta_{i j}$.

The transition probability for a neutrino with flavour $\alpha$ to $\beta$ can be expressed in terms of PMNS mixing matrix elements $U_{i j}$ as,

$$
\begin{aligned}
P_{\nu_{\alpha} \rightarrow \nu_{\beta}} & =\delta_{\alpha \beta}-2 \sum_{i>j} \Im\left[U_{\beta j}^{\star} U_{\alpha j} U_{\beta i} U_{\alpha i}^{\star}\right] \sin \frac{\Delta m_{j i}^{2} L}{2 E} \\
& +4 \sum_{i>j} \Re\left[U_{\beta j}^{\star} U_{\alpha j} U_{\beta i} U_{\alpha i}^{\star}\right] \sin ^{2} \frac{\Delta m_{j i}^{2} L}{4 E}
\end{aligned}
$$

Where $\mathrm{E}$ is the neutrino energy, $\mathrm{L}$ is the distance traveled by a neutrino from its production to its interaction with matter.

Consider oscillations between two flavour of neutrinos, then Eq. 1.15, can be written as a $2 \mathrm{D}$ rotational matrix with a mixing angle $\theta$ between them as, 


$$
U=\left(\begin{array}{cc}
\cos \theta & \sin \theta \\
-\sin \theta & \cos \theta
\end{array}\right)
$$

The appearance probability of $\nu_{\beta}$ from $\nu_{\alpha}$ is

$$
\mathrm{P}_{\substack{(-) \\ \nu_{\alpha} \rightarrow \nu_{\beta}}}=\sin ^{2} 2 \theta_{\alpha \beta} \sin ^{2}\left(\frac{\Delta m_{\alpha \beta}^{2} L}{4 E}\right),
$$

and the disappearance probability of $\nu_{\alpha}$ is expressed as

$$
\mathrm{P}_{\substack{(-) \\ \nu_{\alpha} \rightarrow \nu_{\alpha}}}=1-\sin ^{2} 2 \theta_{\alpha \alpha} \sin ^{2}\left(\frac{\Delta m_{\alpha \beta}^{2} L}{4 E}\right)
$$

As we can see from the oscillation probability equations, oscillations can only happen when $\Delta \mathrm{m}_{i j}^{2}$ is a non zero which is a hint of physics beyond the SM. Also, the frequency term in the sinusoidal equation depends on $\mathrm{L} / \mathrm{E}$ which tells that this changes with the experiment.

The phenomena of neutrino oscillations is primarily governed by the parameters like mass squared difference $\left(\Delta \mathrm{m}_{i j}^{2}\right)$, mixing angles between the neutrino flavours $\left(\theta_{i j}\right)$ and $\mathrm{CP}$ violating phase. The numerous experimental results explained in detail in section 1.8, have confirmed the phenomena of neutrino oscillations between the observed neutrinos and many of the neutrino properties were understood. There are still unravelling puzzles on the neutrino mixing properties which are not answered yet. The mass ordering neutrinos $\left(m_{1}<m_{2}<m_{3}\right.$ or $\left.m_{3}<m_{1}<m_{2}\right)$ shown in fig. 1.6, $\theta_{23}$ octant degeneracy $\left(0<\theta_{23}<45\right.$ degrees or $45<\theta_{23}<90$ degrees $)$ and the value of CP phase $\delta$ is zero or not, are yet to answer.

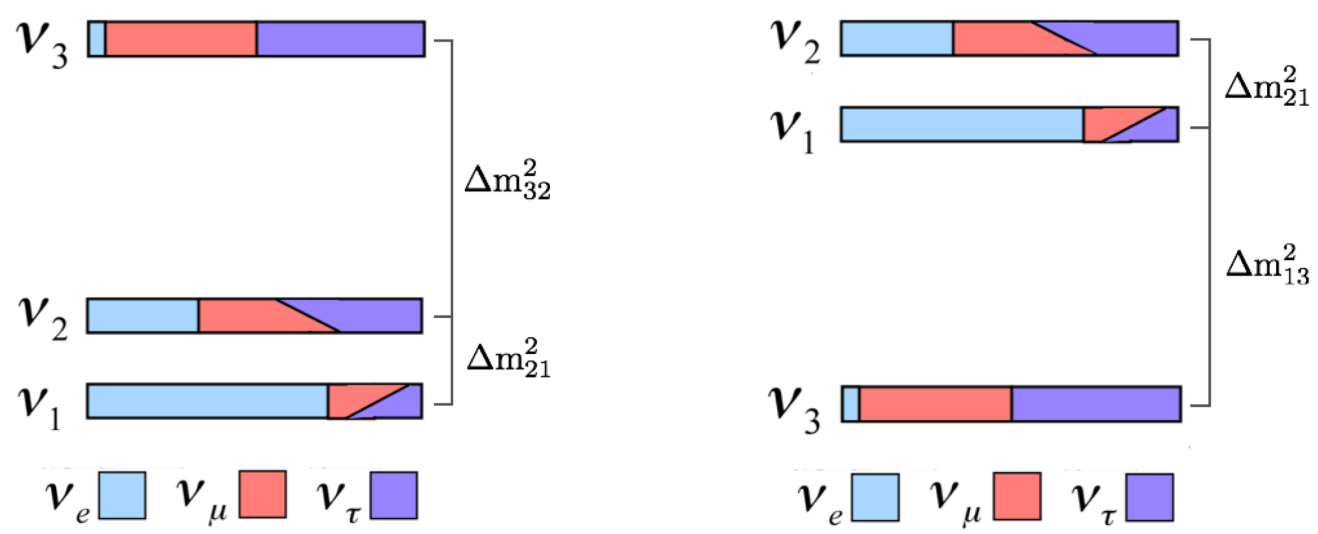

Figure 1.6: The two possible mass orderings of the masses in neutrino 3-flavor model. The color shows the makeup of mass eigen states by the flavour eigen states. 


\subsubsection{Neutrino Oscillations in Matter}

The formalism of neutrino oscillations given in Section 1.7.1 is only valid in a vacuum, but this is not the case in real life, so we must modify the formalism to include the effects of matter. NOvA uses the NuMI neutrino beam which is made using the Accelerator facility at Fermilab. The NOvA Far Detector has located $810 \mathrm{~km}$ away from Fermilab at Ash River, Minnesota, to observe the first neutrino oscillation maxima. Therefore neutrinos have a long passage through the Earth before they reach the far detector. While traveling only electron neutrinos, as they encounter electrons in the earth matter, participate in the coherent charged current forward scattering with electrons, which changes the neutrino oscillation probabilities. The two other flavours of neutrinos, muon and tau neutrino, do not participate in the scattering due to the lack of corresponding partner leptons. In particular, $\nu_{e}$ 's participate in both charged current and neutral current interactions, but $\nu_{\mu}$ 's and $\nu_{\tau}$ 's participate only in neutral current interactions. These neutrinos experience a matter potential $\mathrm{V}= \pm \sqrt{2} \mathrm{G}_{F} \rho_{e}$ due to Earth matter, where $G_{F}$ is the Fermi constant, $\rho_{e}$ is electron number density, and the positive sign is for neutrinos, and the negative sign is for antineutrinos.

In a 2-flavour model, the effective Hamiltonian can be written as follows

$$
\begin{aligned}
H_{M} & =\frac{\Delta m^{2}}{4 E}\left(\begin{array}{cc}
-\cos 2 \theta & \sin 2 \theta \\
\sin 2 \theta & \cos 2 \theta
\end{array}\right)+\left(\begin{array}{ll}
V & 0 \\
0 & 0
\end{array}\right) \\
& =\frac{\Delta m^{2}}{4 E}\left(\begin{array}{cc}
-\cos 2 \theta+A & \sin 2 \theta \\
\sin 2 \theta & \cos 2 \theta-A
\end{array}\right)
\end{aligned}
$$

with

$$
A= \pm \frac{2 \sqrt{2} G_{F} \rho_{e} E}{\Delta m^{2}}
$$

The 2-flavour appearance probability can be written in terms of effective mixing angles and mass-squared differences similar to vacuum oscillation probability as,

$$
\mathrm{P}_{\nu_{\alpha} \rightarrow \nu_{\beta}}=\sin ^{2} 2 \theta_{m} \sin ^{2}\left(\frac{\Delta m_{m}^{2} L}{4 E}\right)
$$

The effective mixing angles and mass-squared differences can be expressed as 


$$
\begin{aligned}
\Delta m_{m}^{2} & =F \Delta m^{2} \\
\sin 2 \theta_{m} & =\frac{\sin 2 \theta}{F} \\
\text { where } \quad F & =\sqrt{(\cos 2 \theta-A)^{2}+\sin ^{2} 2 \theta}
\end{aligned}
$$

- From Eq. 1.24 and 1.25, it is evident that $\sin 2 \theta_{m}=1$ when $\cos 2 \theta=$ A, which is called the resonant condition at which the oscillations significantly enhanced for neutrinos and significantly suppressed for antineutrinos irrespective of the mixing angle $\theta$ in vacuum.

- Neutrinos and antineutrinos have the different oscillation probabilities because of the sign difference as shown in Eq. 1.21 where the plus sign is for neutrinos and minus sign is for antineutrinos. This is true even if CP is not violated in neutrino interactions and even if the mixing matrix is real.

- From Eq. 1.21, resonance happens when A $>0$, where the sign of A depends on the sign of $\Delta m^{2}$ which helps resolve the neutrino mass hierarchy.

\subsection{Experimental Evidence for Neutrino Oscillations}

Many experiments have witnessed the phenomenon of neutrino oscillations. All these experimental results are explained by the neutrino mixing formalism.

- Solar Neutrino Experiments

Solar neutrino experiments are used to study the neutrinos (primarily, electron neutrinos $\nu_{e}$ ) produced in the core of the sun. These experiments can measure the mixing angle $\theta_{12}$ and $\Delta \mathrm{m}_{12}^{2}$ shown in Tab. 1.1. Sun emits the electron neutrinos with energy of the order of $\mathrm{MeV}$ from the different fusion reactions as shown in fig. 1.7. The so called Solar Neutrino Problem was discovered in the Homestake experiment [22]. Experiments with water cherenkov detectors Kamiokande [23, 24] and Super-Kamiokande [31], Sudbury Neutrino Observatory [32, 33], gallium radio-chemical experiments SAGE [34-38], GALLEX [39-43], GNO [44] and liquid scintillation detectors Borexino [45-47] and KamLAND [25, 26]. All of these experiments have confirmed the disappearance of $\nu_{e}$ and $\bar{\nu}_{e}$ coming from the Sun which has now been confirmed as the neutrino oscillation phenomena. It is understood that $\nu_{e}$ 's are oscillating to $\nu_{\mu}$ 's and $\nu_{\tau}$ 's. 
All the solar neutrino experiments have seen the $\bar{\nu}_{e}$ disappearance in the neutrinos from the Sun and KamLAND have seen the disappearance of the reactor neutrino flux. The combined results proved that the Large Mixing Angle (LMA) is the real solution for the solar neutrino problem.

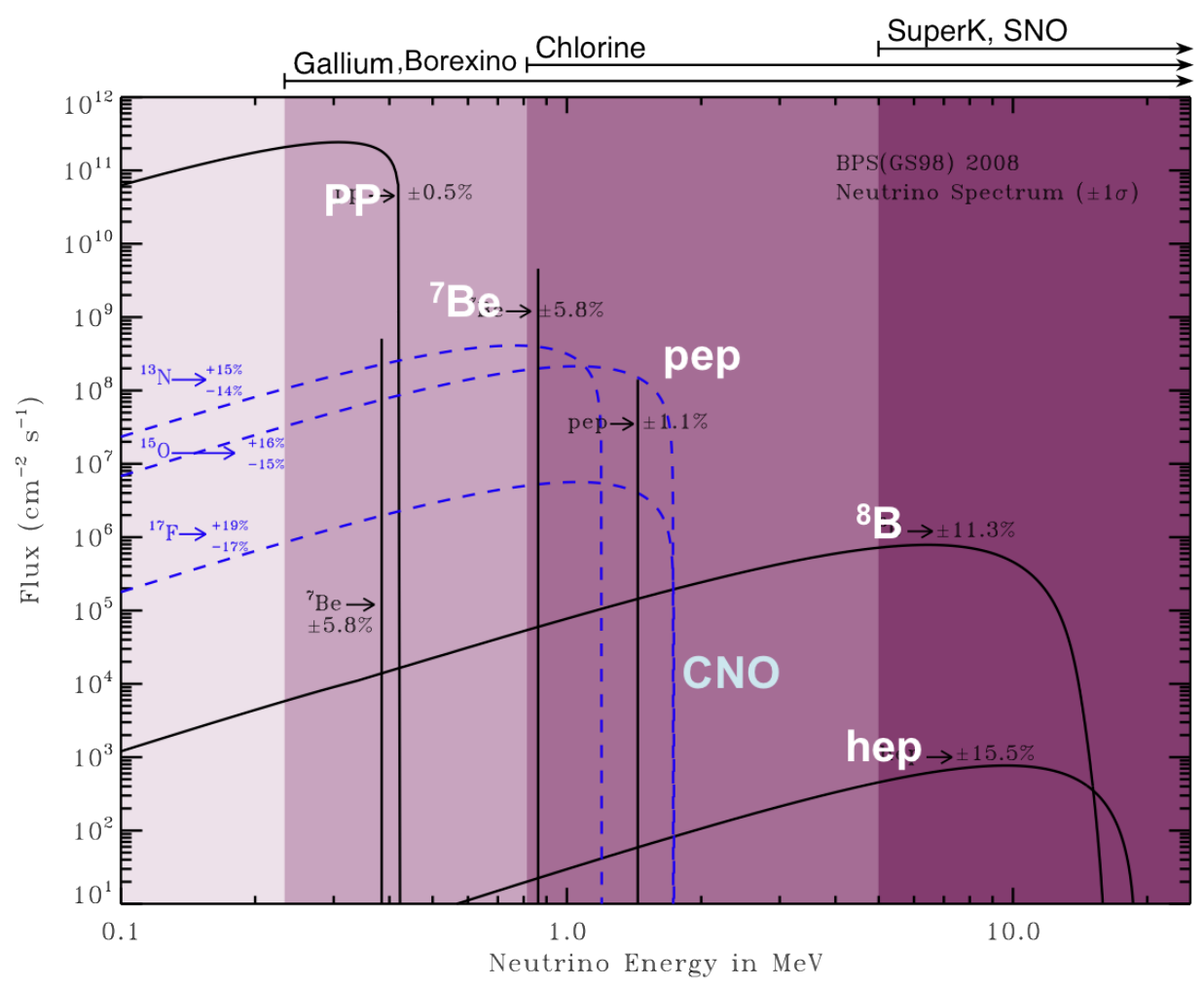

FIGURE 1.7: Energy spectra of neutrino flux from the pp and CNO chains as predicted by the Standard Solar Model [48]. For continuos sources, the differential flux is measured in $\mathrm{cm}^{-2} \mathrm{~S}^{-1} \mathrm{MeV}^{-1}$ and for the lines, it is measured in $\mathrm{cm}^{-2} \mathrm{~s}^{-1}$. This figure is taken from [49].

- Atmospheric Neutrino Experiments

Atmospheric neutrino experiments are used to study the neutrinos produced in the upper atmosphere by the cosmic ray particles. These experiments can measure the mixing angle $\theta_{23}$ and $\Delta \mathrm{m}_{32}^{2}$ shown in Tab. 1.1 by observing the $\nu_{\mu}$ disappearance. Kamiokande [23, 23], IMB [50] Super-Kamiokande [31] and MINOS [51-53], MACRO [54] experiment and ANTARES telescope [55] has observed the muon neutrino disappearance from $\nu_{\mu}$ 's produced in the atmosphere. The results from this experiment were further confirmed by the long-baseline accelerator neutrino experiment $\mathrm{K} 2 \mathrm{~K}$ [56] in Japan. These experiments measure the ratio $\Phi\left(\nu_{\mu}+\bar{\nu}_{\mu}\right) / \Phi\left(\nu_{e}+\bar{\nu}_{e}\right)$.

- Reactor Neutrino Experiments

Reactor neutrino experiments study the neutrinos produced in the fission reactions 
in nuclear reactors. They measure the mixing angle $\theta_{13}$ as shown in Tab. 1.1 by observing the $\nu_{e}$ disappearance. The long-baseline KamLAND [25, 25] $(\sim$ $100 \mathrm{~km}$ ), CHOOZ [57], Double CHOOZ [58], Daya Bay [59, 60] and RENO [61] $(\sim 1 \mathrm{~km})$ have observed strong evidence for the disappearance of $\bar{\nu}_{e}$ using the neutrinos produced in the inverse $\beta$-decay processes in the nuclear reactor. The CHOOZ experiment in France and Palo Verde experiment [62] in Arizona have not observed any $\bar{\nu}_{e}$ disappearance, but the other reactor experiments had seen the disappearance.

- Accelerator Neutrino Experiments Accelerator neutrino experiments study the neutrinos produced by accelerators. These experiments measure the mixing angle $\theta_{23}, \Delta \mathrm{m}_{13}^{2}$ and the CP violating phase as shown in Tab. 1.1 by observing the $\nu_{e}$ appearance and $\nu_{\mu}$ disappearance. Long-baseline experiments like K2K [56], MINOS [51-53], MINOS+ [63, 64], T2K $[65,66]$ and NOvA $[67,68]$ have observed strong evidence of $\nu_{\mu}$ disappearance and $\nu_{e}$ appearance in the neutrino beam produced in a accelerator facility. In addition, experiments like Super-Kamiokande [31], and OPERA [69, 70] have observed the evidence of $\nu_{\mu} \rightarrow \nu_{\tau}$ oscillations. Accelerator experiments are also sensitive to $\delta_{C P}$ and mass hierarchy.

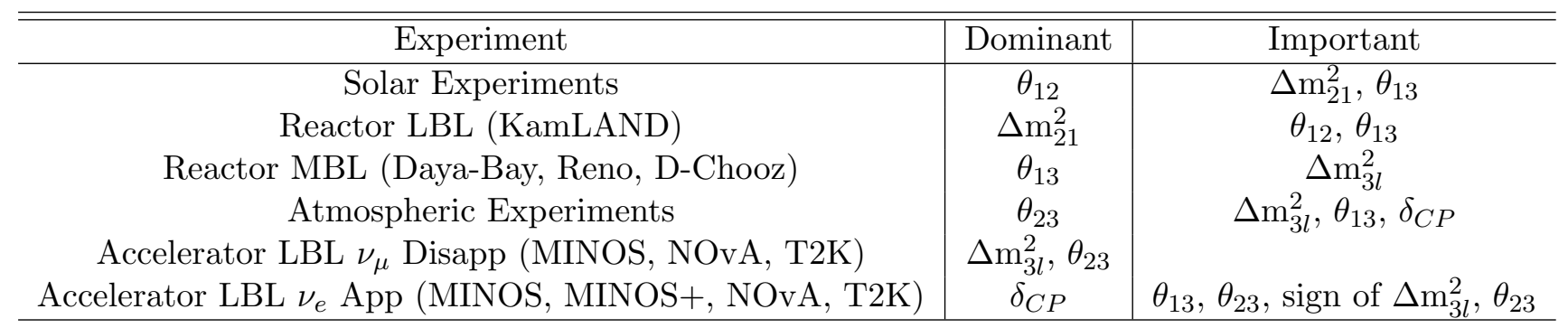

TABLE 1.1: Experimental determination of oscillation parameters by various type of neutrino experiments [71].

\subsubsection{Present Knowledge of Oscillation Parameters}

\subsubsection{Known Parameters}

Numerous experimental results are available on the neutrino mixing parameters. The current knowledge of the mixing parameters is summarized here. These are current best-fit values from the Particle Data Group [72]. 
- $\Delta \mathrm{M}_{21}^{2}$ and $\theta_{12}$

The current best-fit from the solar neutrino experiments is $\Delta \mathrm{M}_{21}^{2}=7.56 \times 10^{-5}$ $\mathrm{eV}^{2}$ and $\theta_{12}=34.5^{0}$ with normal or inverted hierarchy.

- $\Delta \mathrm{M}_{32}^{2}$ and $\theta_{23}$

The current best-fit from the atmospheric neutrino experiments is $\Delta \mathrm{M}_{32}^{2}=2.55 \times$ $10^{-3} \mathrm{eV}^{2}$ and $\theta_{23}=41^{0}$ with normal hierarchy and $\theta_{23}=50.5^{0}$ with inverted hierarchy.

- Mixing angle, $\theta_{13}$

The current global fit from the reactor and accelerator experiments is found to be $\theta_{13}=8.44^{0}$ with normal hierarchy and $\theta_{13}=8.41^{0}$ with inverted hierarchy.

\subsubsection{Unknown Parameters}

There are still unanswered questions with the currently available neutrino data. Some of them include,

- Sign of $\Delta \mathrm{M}_{31}^{2}$ - Mass Hierarchy

Neutrino experiments have not yet found the sign of $\Delta \mathrm{M}_{31}^{2}$. In normal hierarchy, $m_{3}>>m_{2}, m_{1}$ whereas in inverted hierarchy, $m_{3}<<m_{2}, m_{1}$. The matter effects in long-baseline experiments would help resolve this hierarchy problem. This is of critical importance in neutrino-less double $\beta$-decay.

- $\theta_{23}$ Octant

Current $\theta_{23}$ measurement is at 45 degrees which reveal the admixture of the mass state $\nu_{3}$. If it is maximal, then flavour states contain $50 \%$ of $\nu_{\mu}$ and $\nu_{\tau}$. But it is hard to measure alone from muon disappearance experiments.

- CP Violation, $\delta_{C P}$

Long-baseline experiments have the potential to determine the CP-violation phase. If CP violates, $\mathrm{P}\left(\nu_{\alpha} \rightarrow \nu_{\beta}\right) \neq \mathrm{P}\left(\bar{\nu}_{\alpha} \rightarrow \bar{\nu}_{\beta}\right)$ for $\alpha \neq \beta$. The difference between the two probabilities is

$$
P\left(\nu_{\alpha} \rightarrow \nu_{\beta}\right)-P\left(\bar{\nu}_{\alpha} \rightarrow \bar{\nu}_{\beta}\right)=4 \sum_{i>j} \Im\left[U_{\beta j}^{\star} U_{\alpha j} U_{\beta i} U_{\alpha i}^{\star}\right] \sin \frac{\Delta m_{j i}^{2} L}{2 E}
$$

- Nature of the neutrino

Current neutrino experimental results have not revealed any information on the nature of the neutrino whether it is Dirac or Majorana particle. There are experiments which specifically designed to test the nature of neutrino. 
The long-baseline NOvA neutrino experiment is designed to answer some of these questions.

\subsection{Sterile Neutrinos}

Neutrino oscillations are well understood in the three-neutrino framework as was described in the previous sections. But, there are few experiments reported anomalous results that do not fit within the three flavour framework. These anomalies can be explained by introducing a new type of neutrino with mass is the order eV. In 1989, the LEP experiment from the decay width of $\mathrm{Z}$ boson put a constraint that there can be only three generations of leptons with mass less than the mass of $\mathrm{Z} / 2$ which interacts via the weak force. But due to LEP measurement, it can not interact with the weak force, so we call it a sterile neutrino. The minimal extension to the 3 -flavour model is $(3+\mathrm{N})$ model, where $\mathrm{N}$ any number of additional neutrino flavours. Indeed, many of the anomalous results fit well with $\mathrm{N}=1$, referred to as the $(3+1)$ model. The sterile neutrino is considered to be a right-handed neutrino. These neutrinos do not couple with the weak gauge bosons, $\mathrm{W}$ and $\mathrm{Z}$, which implies that there is no constraint on the number of sterile neutrino flavours.

There are even cosmological implications of the existence of sterile neutrinos. The recent data from PLANCK satellite on CMB radiation reveals the potential evidence for more than three neutrino flavours $\left(3.32_{-0.51}^{+0.54}<\mathrm{N}_{\text {eff }}<3.62_{-0.48}^{0.50}\right)$, where $\mathrm{N}_{\text {eff }}$ is effective number of neutrino species [73-75]. Sterile neutrino would also contribute to the sum of the neutrino masses, but there are many conflicts from various models on the sum of neutrino masses, so the current bounds on this don't rule out sterile neutrinos. If we add an additional neutrino to the standard three flavour model, there will be additional three mixing parameters and two phases introduced.

\section{$1.10 \quad(3+1)$ Model}

Most of the neutrino oscillation experimental results explained well within the threeflavour framework. But, the above mentioned anomalous results suggest the existence of an additional flavour of neutrino, called sterile neutrino. The simplest extension to a 3 -flavour model is $(3+1)$ model with one additional sterile neutrino $\left(\nu_{s}\right.$ with the corresponding mass eigen state $\left.\nu_{4}\right)$. The mass ordering of the $(3+1)$ is shown in Fig. 1.8. In principle, there is no restriction on the number of sterile flavours because the sterile neutrino doesn't participate in any of the SM interactions. 
The PMNS mixing matrix can be extended by one row and one column from the standard 3 -flavour matrix to incorporate one additional sterile flavour. So, the mixing matrix for $(3+1)$ model can be written as

$$
\left(\begin{array}{c}
\nu_{e} \\
\nu_{\mu} \\
\nu_{\tau} \\
\nu_{s}
\end{array}\right)=\left(\begin{array}{cccc}
\mathrm{U}_{e 1} & \mathrm{U}_{e 2} & \mathrm{U}_{e 3} & \mathrm{U}_{e 4} \\
\mathrm{U}_{\mu 1} & \mathrm{U}_{\mu 2} & \mathrm{U}_{\mu 3} & \mathrm{U}_{\mu 4} \\
\mathrm{U}_{\tau 1} & \mathrm{U}_{\tau 2} & \mathrm{U}_{\tau 3} & \mathrm{U}_{\tau 4} \\
\mathrm{U}_{s 1} & \mathrm{U}_{s 2} & \mathrm{U}_{s 3} & \mathrm{U}_{s 4}
\end{array}\right)\left(\begin{array}{c}
\nu_{1} \\
\nu_{2} \\
\nu_{3} \\
\nu_{4}
\end{array}\right)
$$

where $\nu_{s}$ and $\nu_{4}$ are the flavor and mass eigen states of sterile neutrino respectively, and $\mathrm{U}_{\alpha 4}, \alpha=e, \mu, \tau, s$ represents the mixing between active and sterile neutrino.

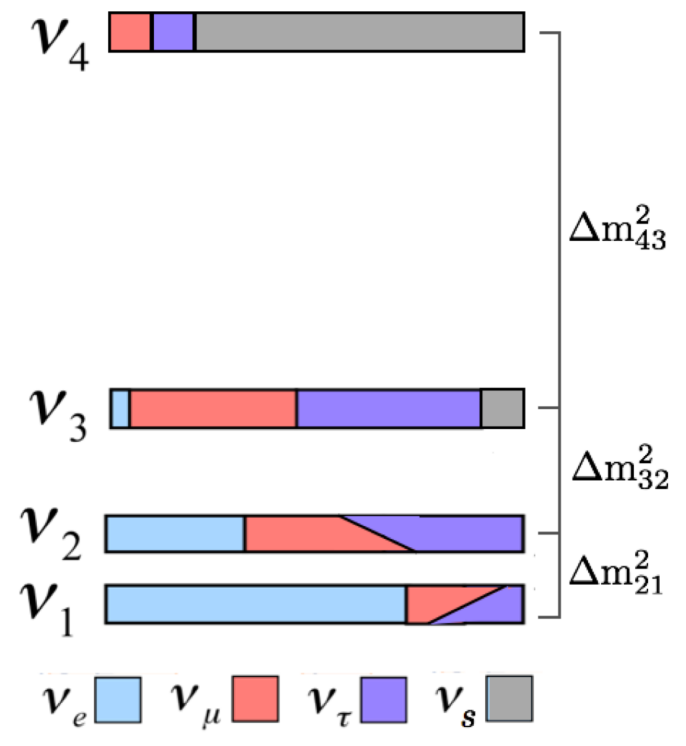

FiguRE 1.8: $(3+1)$ flavor model

Anomalous results from the short-baseline experiments indicate that the mass squareddifference between active and sterile neutrino is of the order $1 \mathrm{eV}^{2}$ which indicates the the mass eigen state $\nu_{4}$ is heavier than the standard mass eigen states $\left(\nu_{1}, \nu_{2}, \nu_{3}\right)$. The neutrino oscillation experiments have measured the solar and atmospheric mass squareddifference among the active neutrinos as $\Delta \mathrm{m}_{\text {solar }}^{2}\left(\Delta \mathrm{m}_{21}^{2}\right) \approx 7.53 \times 10^{-5} \mathrm{eV}^{2}$ and $\Delta \mathrm{m}_{\text {atm }}^{2}$ $\left(\Delta \mathrm{m}_{32}^{2}\right) \approx 2.42 \times 10^{-3} \mathrm{eV}^{2}[72]$.

In Quantum mechanics, any physical state can be expressed as a superposition of basis states. Similarly, a neutrino of a definite flavour state can be written as a coherent superposition of mass eigen states as follows 


$$
\left|\nu_{\alpha}(0)\right\rangle=\sum_{i} U_{i \alpha}^{\star}\left|\nu_{i}\right\rangle
$$

where the index $\alpha$ denotes the flavour states $(e, \mu, \tau)$ and $i$ denotes the mass eigen states and runs over three mass states.

The time evolution at $(x, t)$ can be written as,

$$
\begin{aligned}
\left|\nu_{\alpha}(t)\right\rangle & =\sum_{i} U_{i \alpha}^{\star} e^{i p_{i} \cdot x}\left|\nu_{i}\right\rangle \\
& =\sum_{i} U_{i \alpha}^{\star} e^{i\left(E_{i} t-p_{i} x\right)}\left|\nu_{i}\right\rangle
\end{aligned}
$$

where $E_{i}$ and $p_{i}$ are energy and momentum of a neutrino mass eigen state $i$ at $(\vec{x}, t)$ and $p_{i} . x$ is a scalar product of space-time four-vector and momentum four-vector. The transition probability of a neutrino with flavour $\alpha$ at $(0,0)$ to be found as a neutrino with flavour $\beta$ at $(\vec{x}, \mathrm{t})$ can be obtained as a square of the amplitude.

$$
P_{\nu_{\alpha} \rightarrow \nu_{\beta}}=\left|\left\langle\nu_{\beta} \mid \nu_{\alpha}(t)\right\rangle\right|^{2}
$$

where

$$
\left\langle\nu_{\beta} \mid \nu_{\alpha}(t)\right\rangle=\sum_{j} \sum_{i} U_{\beta j} U_{\alpha i}^{\star} e^{i p_{i} . x}\left\langle\nu_{j} \mid \nu_{i}\right\rangle
$$

when we sum over $j$ index,

$$
\left\langle\nu_{\beta} \mid \nu_{\alpha}(t)\right\rangle=\sum_{i} U_{\beta j} U_{\alpha i}^{\star} e^{i p_{i} \cdot x}
$$

The 4-vector product can be expanded as

$$
p_{i} \cdot x=E_{i} t-\overrightarrow{p_{i}} \cdot \vec{x}
$$

The 3-vector product can be written as 


$$
\begin{aligned}
\vec{p} \cdot \vec{x} & =\left|\overrightarrow{p_{i}}\right||\vec{x}| \cos (0) \\
& =\left|\overrightarrow{p_{i}}\right| L
\end{aligned}
$$

Where $L$, the distance traveled by a neutrino mass eigen state $i$. From Einstein's massenergy relationship, we can simply express energy in terms of mass and momentum, as $E_{i}=\sqrt{p_{i}^{2}+m_{i}^{2}}$. We can assume $t=L$ because neutrino is moving with a velocity close speed of light.

So, Eq. 1.34 now becomes

$$
\begin{aligned}
p_{i} . x & =L\left(\sqrt{p_{i}^{2}+m_{i}^{2}}\right)-\left|p_{i}\right| L \\
& =\left|p_{i}\right| L\left(\frac{m_{i}^{2}}{2 p_{i}^{2}}\right) \\
& =\frac{m_{i}^{2} L}{2 E}
\end{aligned}
$$

thus

$$
\left\langle\nu_{\beta} \mid \nu_{\alpha}(t)\right\rangle=\sum_{i} U_{\beta j} U_{\alpha i}^{*} e^{i \frac{m_{i}^{2} L}{2 E}}
$$

Hence the probability becomes,

$$
\begin{aligned}
P_{\nu_{\alpha} \rightarrow \nu_{\beta}} & =\left(\sum_{j} U_{\beta j}^{*} U_{\alpha j} e^{-i \frac{m_{j}^{2} L}{2 E}}\right)\left(\sum_{i} U_{\beta i} U_{\alpha i}^{*} e^{i \frac{m_{i}^{2} L}{2 E}}\right) \\
& =\sum_{i} \sum_{j} U_{\beta j}^{*} U_{\alpha j} U_{\beta i} U_{\alpha i}^{*} \exp \left(-i \frac{\Delta m_{i j}^{2} L}{2 E}\right)+\sum_{i} \sum_{j} U_{\alpha i}^{*} U_{\beta j} U_{\alpha i} U_{\beta j}^{*} \\
& -\sum_{i} \sum_{j} U_{\alpha i}^{*} U_{\beta j} U_{\alpha i} U_{\beta j}^{*} \\
& =\sum_{i} \sum_{j} U_{\beta j}^{\star} U_{\alpha j} U_{\beta i} U_{\alpha i}^{\star}+\sum_{i} \sum_{j} U_{\beta j}^{\star} U_{\alpha j} U_{\beta i} U_{\alpha i}^{\star}\left[\exp \left(-i \frac{\Delta m_{j i}^{2} L}{2 E}\right)-1\right]
\end{aligned}
$$

The unitary matrix satisfies the identity, 


$$
\begin{gathered}
U^{\dagger} U=I \Rightarrow \sum_{i} U_{\alpha i} U_{\beta i}^{*}=\delta_{\alpha \beta} \\
\sum_{i} \sum_{j} U_{\beta j}^{\star} U_{\alpha j} U_{\beta i} U_{\alpha i}^{\star}=\sum_{i} U_{\beta i} U_{\alpha i}^{\star} \sum_{j} U_{\alpha j} U_{\beta j}^{\star} \\
=\delta_{\alpha \beta}
\end{gathered}
$$

We can evaluate the second term in Eq. 1.41. When $i=j$, this evaluates to 0. But, when $i>j$, it will be conjugate when $i<j$. Let $A$ is a complex number, then $A+A^{*}=2 \Re(A)$.

$$
\sum_{i} \sum_{j} U_{\beta j}^{\star} U_{\alpha j} U_{\beta i} U_{\alpha i}^{\star}\left[\exp \left(-i \frac{\Delta m_{j i}^{2} L}{2 E}\right)-1\right]=\sum_{i>j} 2 \Re\left[U_{\beta j}^{\star} U_{\alpha j} U_{\beta i} U_{\alpha i}^{\star}\left[\exp \left(-i \frac{\Delta m_{j i}^{2} L}{2 E}\right)-1\right]\right]
$$

Then, Eq. 1.41 becomes,

$$
P_{\nu_{\alpha} \rightarrow \nu_{\beta}}=\delta_{\alpha \beta}+\sum_{i>j} 2 \Re\left[U_{\beta j}^{\star} U_{\alpha j} U_{\beta i} U_{\alpha i}^{\star}\left[\exp \left(-i \frac{\Delta m_{j i}^{2} L}{2 E}\right)-1\right]\right]
$$

Now the exponential term is expanded using the identity,

$$
\begin{gathered}
\exp \left(-i \frac{\Delta m_{j i}^{2} L}{2 E}\right)-1=\left(\cos \frac{\Delta m_{j i}^{2} L}{2 E}-1\right)-i \sin \frac{\Delta m_{j i}^{2} L}{2 E} \\
=2 \sin ^{2} \frac{\Delta m_{j i}^{2} L}{4 E}-i \sin \frac{\Delta m_{j i}^{2} L}{2 E} \\
P_{\nu_{\alpha} \rightarrow \nu_{\beta}}=\delta_{\alpha \beta}-2 \sum_{i>j} \Im\left[U_{\beta j}^{\star} U_{\alpha j} U_{\beta i} U_{\alpha i}^{\star}\right] \sin \frac{\Delta m_{j i}^{2} L}{2 E} \\
+4 \sum_{i>j} \Re\left[U_{\beta j}^{\star} U_{\alpha j} U_{\beta i} U_{\alpha i}^{\star}\right] \sin ^{2} \frac{\Delta m_{j i}^{2} L}{4 E}
\end{gathered}
$$




\subsection{1 $\nu_{e}$ Appearance and $\nu_{\mu}$ Disappearance Probability}

When considering only oscillations over short distances then $\Delta \mathrm{m}_{21}^{2}$ and $\Delta \mathrm{m}_{32}^{2}$ are negligible. We can use this to simplify Eq. 1.47. Then the only terms which remain non-zero in Eq. 1.47 are terms with $i>3$. If we expand the $\nu_{\mu} \rightarrow \nu_{e}$ appearance probability from Eq. 1.47 can be written as

$$
\begin{aligned}
& \mathrm{P}_{\substack{(-) \\
\nu_{\mu} \rightarrow \nu_{e}}}^{\mathrm{SBL}, 3+1}=4\left|\mathrm{U}_{\mu 4}\right|^{2}\left|\mathrm{U}_{e 4}\right|^{2} \sin ^{2} \frac{\Delta m_{41}^{2} L}{4 E}=\sin ^{2} 2 \theta_{\mu e} \sin ^{2} \frac{\Delta m_{41}^{2} L}{4 E} \\
& =\sin ^{2} 2 \theta_{14} \sin ^{2} \theta_{24} \sin ^{2} \frac{\Delta m_{41}^{2} L}{4 E}
\end{aligned}
$$

where $\left|\mathrm{U}_{e 4}\right|=\sin \theta_{14},\left|\mathrm{U}_{\mu 4}\right|=\cos \theta_{14} \sin \theta_{24},\left|\mathrm{U}_{\tau 4}\right|=\cos \theta_{14} \cos \theta_{24} \sin \theta_{34}$ and

$$
\sin ^{2} 2 \theta_{\mu e} \equiv 4\left|\mathrm{U}_{\mu 4}\right|^{2}\left|\mathrm{U}_{e 4}\right|^{2}=\sin ^{2} 2 \theta_{14} \sin ^{2} \theta_{24}
$$

Similarly, $\nu_{\mu} \rightarrow \nu_{\mu}$ disappearance probability from Eq. [1.47] can be written

$$
\underset{\substack{(-) \\ \nu_{\mu} \rightarrow \nu_{\mu}}}{\mathrm{SBL}, 3+1}=1-4\left|\mathrm{U}_{\mu 4}\right|^{2}\left(1-\left|\mathrm{U}_{\mu 4}\right|^{2}\right) \sin ^{2} \frac{\Delta m_{41}^{2} L}{4 E}=1-\sin ^{2} 2 \theta_{\mu \mu} \sin ^{2} \frac{\Delta m_{41}^{2} L}{4 E}
$$

where $\sin ^{2} 2 \theta_{\mu \mu} \equiv 4\left|\mathrm{U}_{\mu 4}\right|^{2}\left(1-\left|\mathrm{U}_{\mu 4}\right|^{2}\right)$ and $\sin ^{2} 2 \theta_{\mu \mu}=\cos ^{2} \theta_{14} \sin ^{2} \theta_{24}$

\subsection{Experimental Evidence For Short-Baseline Oscilla- tions}

Neutrino oscillations at a short-baseline length of the order of few meters can happen if the mass of the sterile neutrino is higher than the mass of the SM neutrinos. The experiments like LSND [76], MiniBooNE [77] reported the excess of neutrinos beyond what they predicted which can be interpreted as oscillations at these short-baseline lengths. In addition, reactor experiments [78-80] with baseline $<100 \mathrm{~m}$ and radiochemical Gallium experiments GALLEX [39-43] and SAGE [34-38] observed a deficiency in the anti-electron neutrino interaction rate. These results can also be explained by introducing one or more additional sterile neutrinos. 


\subsubsection{LSND and MiniBooNE Anomalies}

LSND (Liquid Scintillator Neutrino Detector) [76] aimed to probe neutrino oscillations at short length scales. It was the first experiment to report evidence of neutrino oscillations with $\Delta \mathrm{m}^{2} \geq 1 \mathrm{eV}^{2}$. It was designed to use the neutrino beam from Los Alamos Meson Physics Facility (LAMPF) at Los Alamos National Laboratory and was placed at a distance of $28 \mathrm{~m}$ away from the source. LSND detector was cylindrical with a length of $8.3 \mathrm{~m}$ and diameter of $5.7 \mathrm{~m}$. The tank was filled with 167 tons of liquid scintillator, consisting mineral oil as a solvent and $0.031 \mathrm{~g} /$ liter p-PBD as a scintillant. It is instrumented with 1220 Photo-multiplier tubes, of an 8-inch width, located around the tank covering almost $1 / 4$ of the total surface area of the tank, to capture the Cherenkov light.

LSND used a beam of protons with $798 \mathrm{MeV}$ fired on to a carbon target which emits secondary mesons such as pions. These pions decay-at-rest to give muons and muon neutrinos by two body decay as shown in Eq. 1.51 .

$$
\begin{aligned}
& \pi^{ \pm} \rightarrow \mu^{ \pm}+\nu_{\mu}\left(\bar{\nu}_{\mu}\right) \\
& \mu^{ \pm} \rightarrow \bar{\nu}_{\mu}\left(\nu_{\mu}\right)+\nu_{e}\left(\bar{\nu}_{e}\right)+e^{ \pm}
\end{aligned}
$$

The emitted muons further decay to give muon anti-neutrinos and electron neutrinos as sown in Eq. 1.51. The emitted muon anti-neutrinos constitutes the neutrino beam. When a neutrino traverses through the detector, it interacts with the nuclear medium (neutrons, protons) in the liquid scintillator. The emitted particles travel at speeds greater than a speed of light in the medium and then the medium emits Cherenkov radiation. The pulse height and time recorded by PMT are used to reconstruct the neutrino interactions.

LSND searched for Charged Current Quasi Elastic interactions (inverse $\beta$-decay) induced by the anti election neutrino as shown in Eq. 1.53. The selected $\bar{\nu}_{e}$ sample have beam $\nu_{e}$ 's as background. But, the signal consisted of a positron ring along with the emission of a $\gamma$-photon with energy $2.2 \mathrm{MeV}$ by the neutron capture, whereas the beam $\nu_{e}$ just emits one electron.

$$
\bar{\nu}_{e}+\text { proton } \rightarrow \text { neutron }+e^{+}
$$

LSND observed an excess of $87.9 \pm 22.4 \pm 6.0$ events with a background of $19.5 \pm 3.9$ from $\mu^{-}$decay at rest as shown in Fig. 1.9 and $10.5 \pm 4.6$ from the $\pi^{-}$decay at rest. This excess corresponds to $\nu_{\mu} \rightarrow \nu_{e}$ oscillation probability of $(0.264 \pm 0.067 \pm 0.045) \%$ at $3.8 \sigma$ significance. The corresponding results are shown in $\left(\sin ^{2} 2 \theta, \Delta \mathrm{m}^{2}\right)$ plane shown in Fig. 1.10. This hints the existence of neutrino oscillations at short-baseline with the best fit at $\left(\sin ^{2} 2 \theta, \Delta \mathrm{m}^{2}\right) \sim\left(0.003,1.2 \mathrm{eV}^{2}\right)$. 

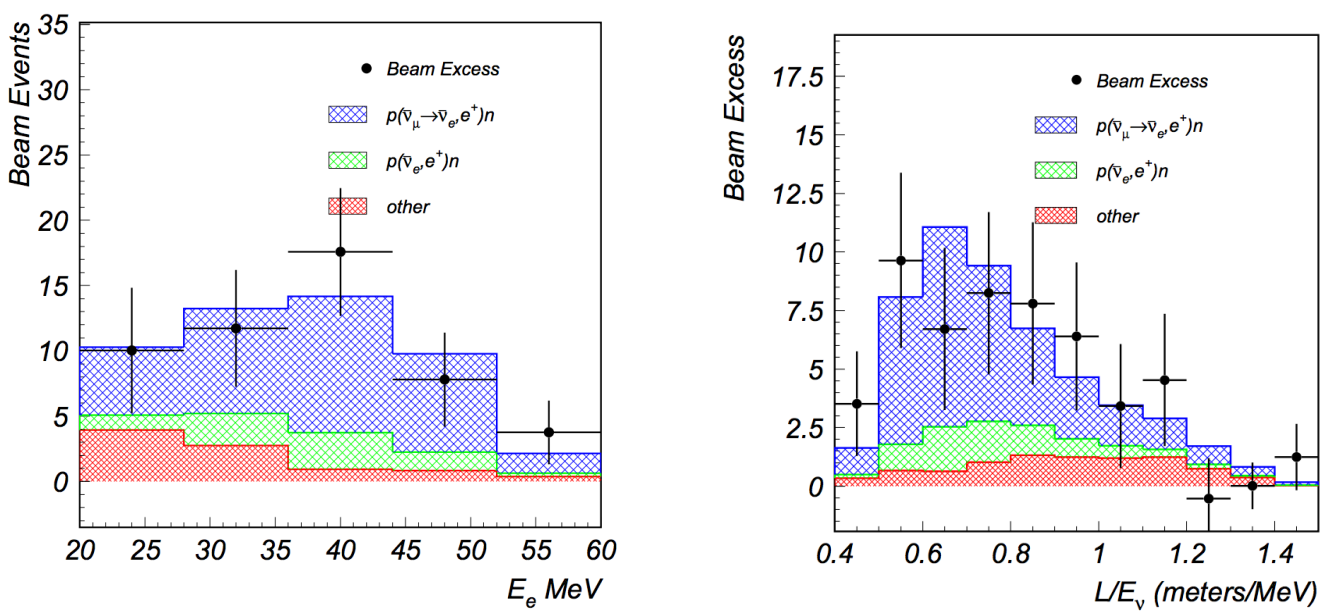

FiguRE 1.9: Left: Shows the energy distribution of the events selected by LSND. Right: Shows the $L / E$ distribution of the selected events. Ref. [76].

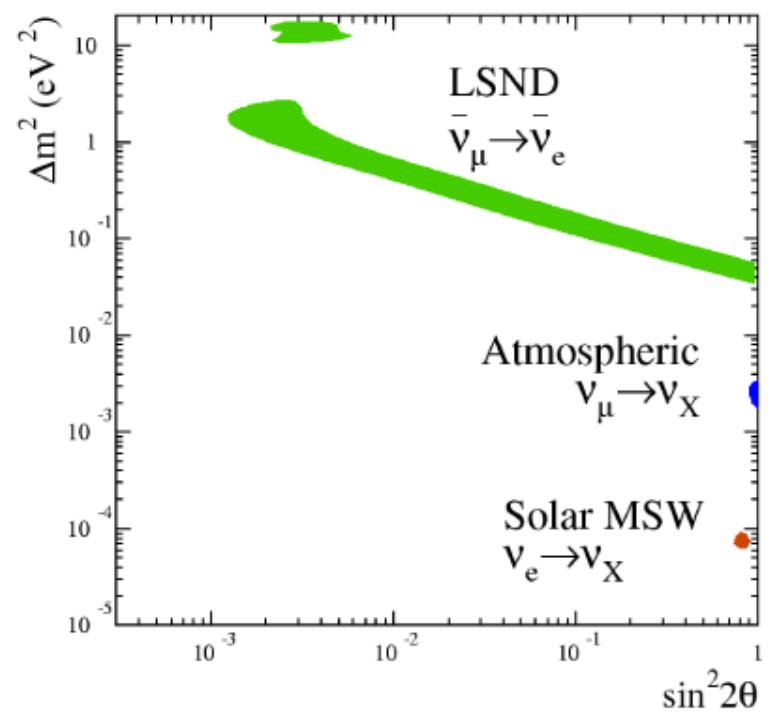

Figure 1.10: Shows LSND results in the $\left(\sin ^{2} 2 \theta, \Delta \mathrm{m}^{2}\right)$ plane at $90 \%$ and $99 \%$ C.L.

\subsubsection{MiniBooNE Anomaly}

MiniBooNE (Mini Booster Neutrino Experiment) [77] was designed to confirm the LSND results. It was placed at Fermilab using the neutrinos from the Booster $8 \mathrm{GeV}$ accelerator ring. Neutrinos are produced by pions which decay in flight. It is placed at $L / E$ same as that of LSND but using different L and E.

A proton beam of $8 \mathrm{GeV}$ from the Booster impinges on to Be-target, $71 \mathrm{~cm}$ long, which then emits secondary mesons like pions and kaons. The mesons are focused by magnetic horns with changeable polarity and fed into a decay pipe of $50 \mathrm{~m}$. The detector is placed 
$541 \mathrm{~m}$ away from the Be target. The neutrino beam contains about $0.6 \%$ intrinsic $\nu_{e}$ beam as shown in Fig. 1.11.
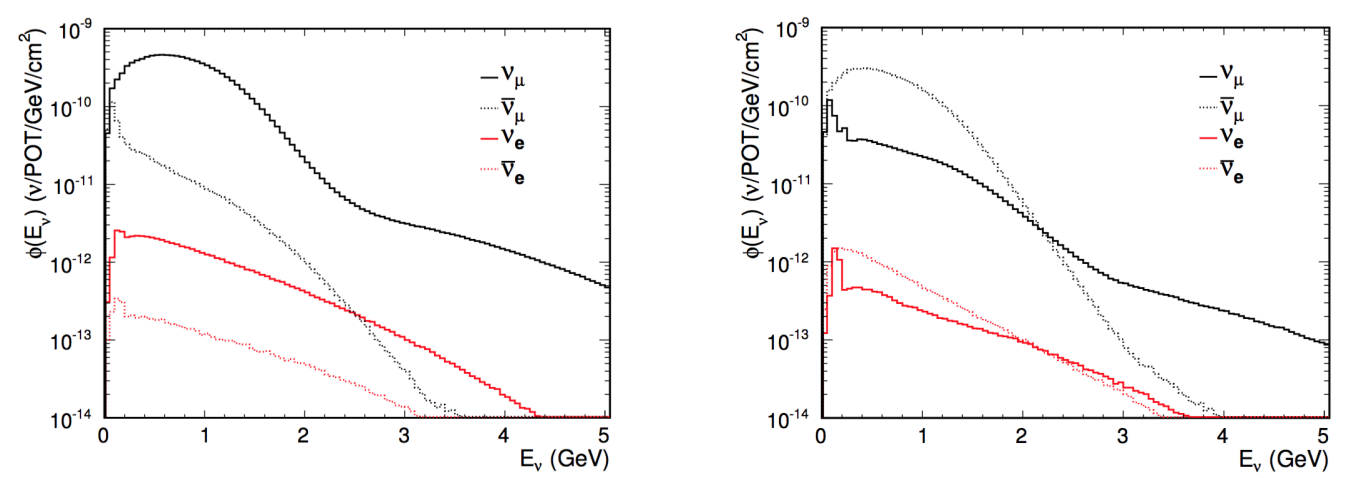

Figure 1.11: Left: Shows the neutrino flux in the neutrino mode at MiniBooNE; Right: Shows the neutrino flux in the anti neutrino mode. Ref. [77].

MiniBooNE consists of a spherical tank of a $12.2 \mathrm{~m}$ diameter filled with liquid scintillator. Its surface is equipped with 1280 PMTs of 8 -inch diameter covering $10 \%$ of its target region.

The designed goal of MiniBooNE was to search for $\nu_{e}\left(\bar{\nu}_{e}\right)$ appearance. The dominant signal is Charged Current Quasi-Elastic interactions, with single photon from NC interactions $\left(\mathrm{NC} \pi^{0} \mathrm{~s}\right)$ as the primary background. These neutrinos interact with the nucleons via $\nu_{e}+n \rightarrow e^{-}+p$ or $\bar{\nu}_{e}+p \rightarrow e^{+}+n$. The signal is identified by studying the characteristic features of the Cherenkov rings.

MiniBooNE observed no excess in the neutrino mode at energies above $475 \mathrm{MeV}$. Instead, it observed an unexplained excess below $475 \mathrm{MeV}$. In the anti-neutrino mode, it found the same excess above $475 \mathrm{MeV}$ as had been seen in LSND, as shown in Fig. 1.12. The corresponding results are shown in Fig. 1.13 in the $\left(\sin ^{2} 2 \theta, \Delta \mathrm{m}^{2}\right)$ plane for both neutrino and anti-neutrino mode.

\subsubsection{GALLIUM Anomaly}

The GALLEX (Gallium Experiment) [39-43] and SAGE (Soviet-American Gallium Experiment) [34-38] solar detectors were designed to address the solar neutrino problem, i.e., to understand why flux of neutrinos smaller than predicted. The GALLEX detector was a cylindrical tank filled with 30.3 tons of Gallium in the form of $\mathrm{GaCl}_{3}-\mathrm{HCl}$. When an electron neutrino interacted with $\mathrm{Ga}$, it produced Ge which formed the volatile $\mathrm{GeCl}_{4}$. It was then collected in the water using the nitrogen stream which changes to $\mathrm{GeH}_{4}$ and introduced into a proportional counter along with Xenon to count the ${ }^{71} \mathrm{Ge}$ atoms by their radioactivity. 

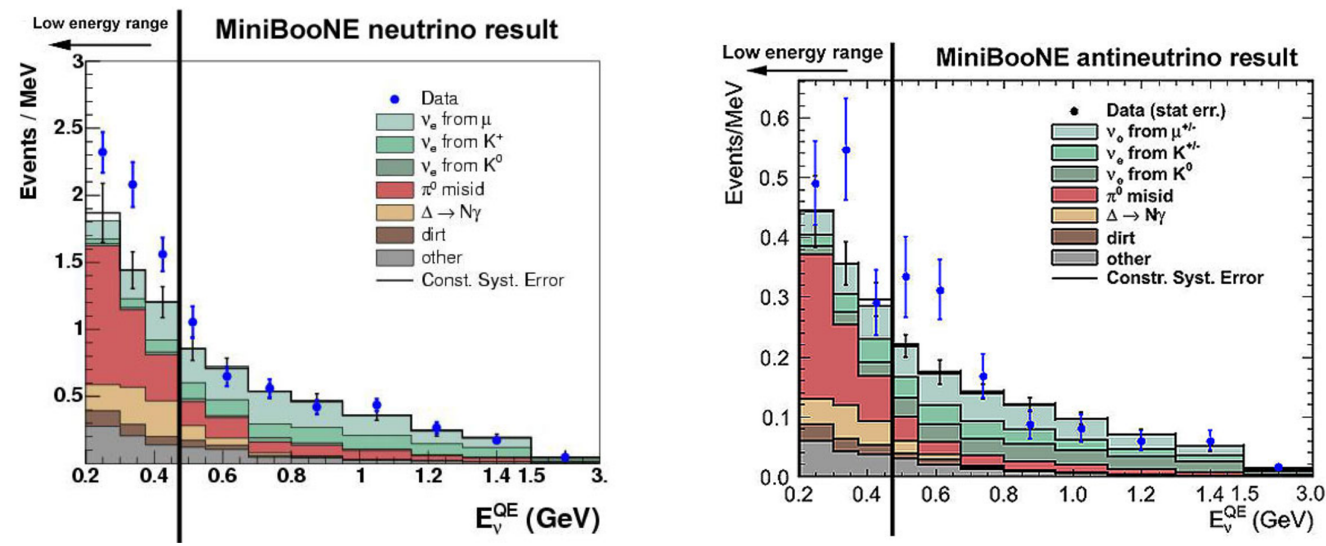

FiguRE 1.12: Left: Shows the selected event rates in the neutrino mode at MiniBooNE. Right: Shows the selected event rates in the anti-neutrino mode. The vertical lines placed at the energy of $475 \mathrm{MeV}$.

While testing the functionality and calibrating these detectors, physicists placed intense radioactive sources inside the detectors. These sources were placed a very short-baseline lengths of just a few meters and detected the electron neutrinos emitted by these radioactive sources using the reaction Eq. 1.54.

$$
\nu_{e}+{ }^{71} \mathrm{Ga} \rightarrow{ }^{71} \mathrm{Ge}+e^{-}
$$

These experiments reported the ratio between the measured and expected ${ }^{37} \mathrm{Ge}$ event rates to be consistently less than unity, as shown in Eq. 1.55. These ratios show a $2.8 \sigma$ deficit in the measured event rate even after considering the uncertainty on the detection cross-section on Gallium. Later, this was named as Gallium Anomaly, and it can also be interpreted as evidence for sterile neutrinos.

The ratio, $\mathrm{R}$, measured were

$$
\begin{aligned}
& R_{B}^{G 1}=0.953 \pm 0.11 \\
& R_{B}^{G 2}=0.812_{-0.11}^{+0.10} \\
& R_{B}^{S 1}=0.95 \pm 0.12 \\
& R_{B}^{S 2}=0.791_{-0.078}^{+0.084}
\end{aligned}
$$

where G1 and G2 are the two GALLEX experiments with ${ }^{51} \mathrm{Cr}$ source, S1 and S2 are SAGE experiment with ${ }^{51} \mathrm{Cr}$ source and ${ }^{37} \mathrm{Ar}$. 


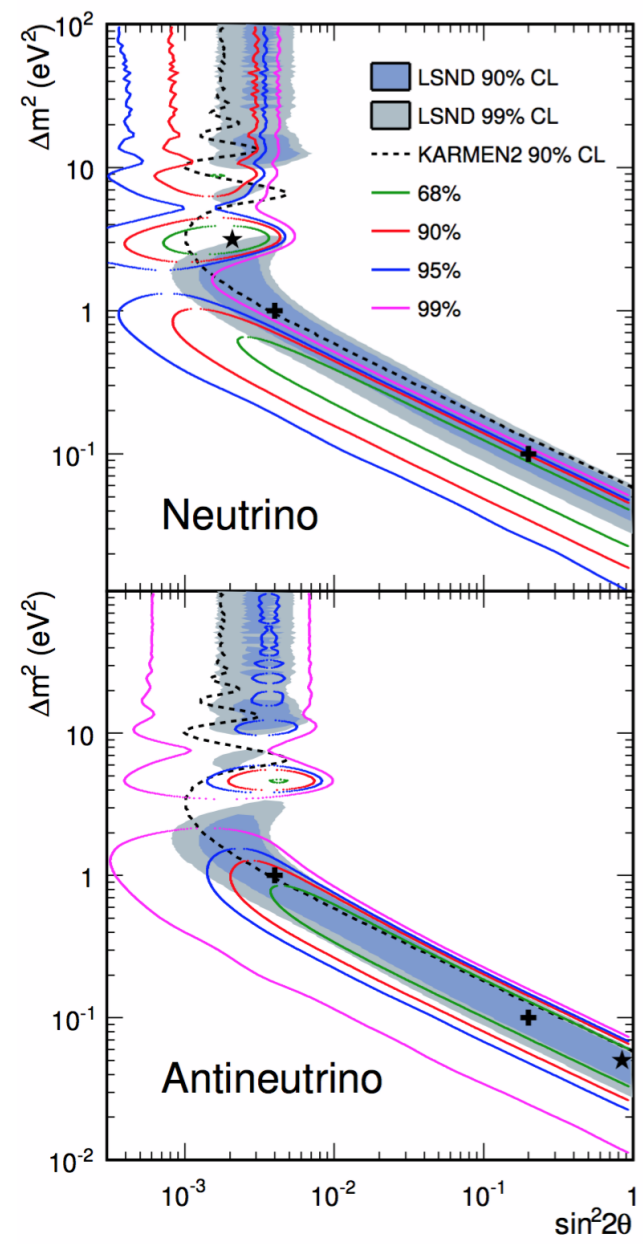

Figure 1.13: Shows the results in the $\left(\sin ^{2} 2 \theta, \Delta \mathrm{m}^{2}\right)$ plane at $90 \%$ and $99 \%$ C.L. As we see MiniBooNE in the neutrino modes excludes some of the LSND region at various significance levels. Also, shown the results from KARMEN [81].

Interpreting these as due to sterile neutrinos, we can place limits in $\left(\sin ^{2} 2 \theta, \Delta \mathrm{m}^{2}\right)$ space as shown in Figure. 1.14.

\subsubsection{Reactor Anomaly}

Short-baseline reactor experiments with a baseline $<100 \mathrm{~m}$ have also observed a deficit in the incoming electron neutrino flux at $2.7 \sigma$ confidence level after the careful consideration of systematic errors. This is called the Reactor Anomaly. Reactors are pure intense source of anti-electron neutrinos emitting $2 \times 10^{20} \nu / \mathrm{s}$ at $4 \pi$ solid angle. Reactor neutrino experiments, ILL - Grenoble [82], Goesgen [83], Rovno [80], Krasnoyarsk [84], Savannah River [85] and Bugey $[78,79]$ are placed $<100 \mathrm{~m}$ from the reactor core.

Updated flux predictions calculated in 2016, looking at $\bar{\nu}_{e}$ flux from the radio-active decays of ${ }^{235} \mathrm{U},{ }^{238} \mathrm{U},{ }^{239} \mathrm{Pu}$ and ${ }^{241} \mathrm{Pu}$. Surprisingly, this new flux was $+3 \%$ higher than 


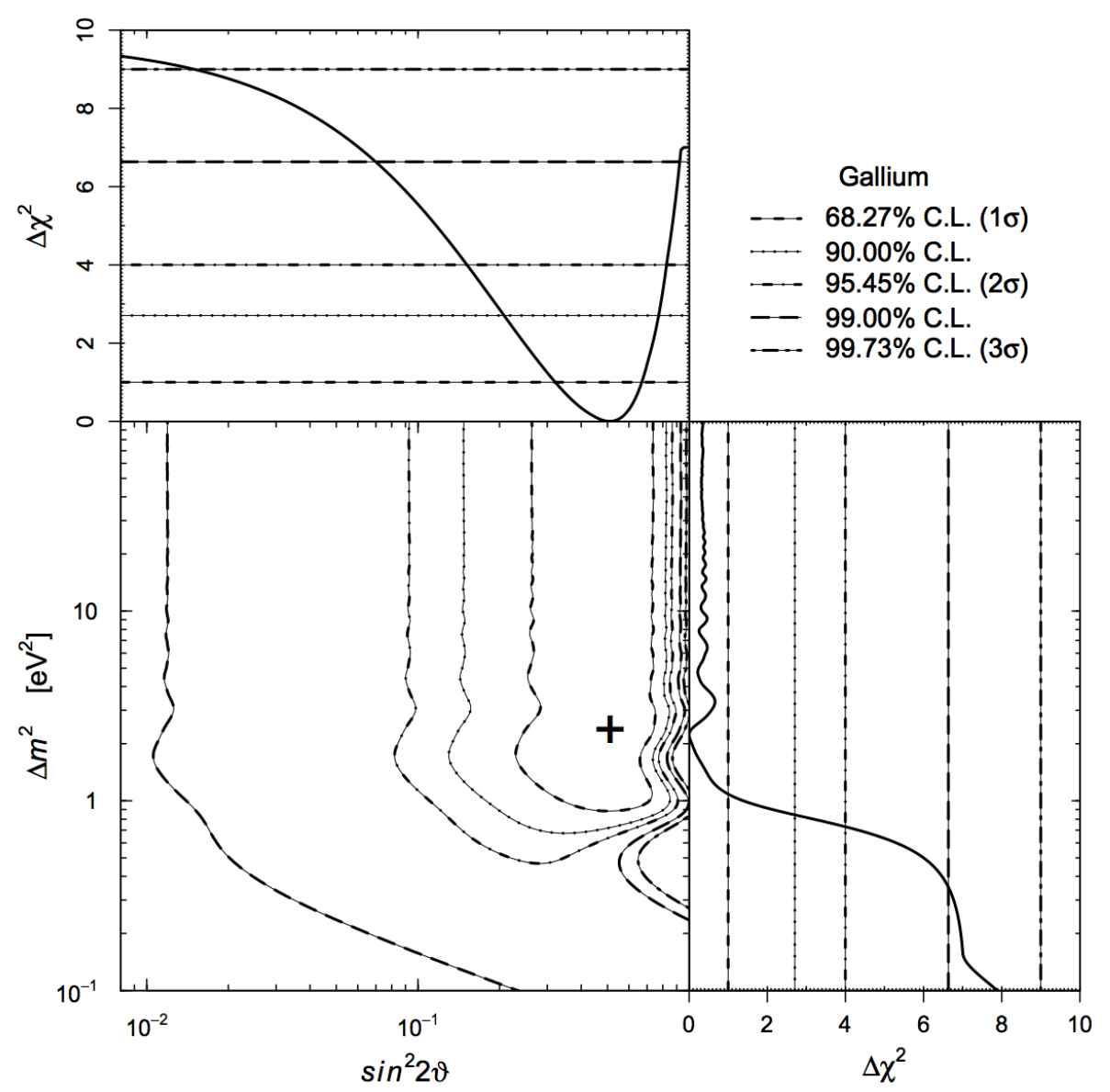

FIGURE 1.14: Shows the combined results from GALLEX and SAGE experiments in the $\left(\sin ^{2} 2 \theta, \Delta \mathrm{m}^{2}\right)$ plane at various C.L. Ref. [34-43].

the previous one. The ratio between measured and predicted to $\mathrm{R}=0.927 \pm 0.023$ as shown in Fig. 1.15. These results can also be considered to be due to the existence of sterile neutrino with mass $\Delta \mathrm{m}^{2}>1 \mathrm{eV}^{2}$. The global-fit from all the reactor experiments shown in Fig. 1.15. The global best-fit determines best fit of $\sin ^{2}(2 \theta)=0.14 \pm 0.08$ (95\% C.L.) and $\left|\Delta \mathrm{m}^{2}\right|>1.5 \mathrm{eV}^{2}$ (95\% C.L.) which disfavors the no sterile neutrino hypothesis at $99.8 \%$ C.L [86]. The corresponding results are shown in Fig. 1.16 in the $\left(\sin ^{2} 2 \theta, \Delta \mathrm{m}^{2}\right)$ plane along with $\Delta \chi^{2}$ distribution at the best fit oscillation parameters.

\subsubsection{Current Status of $(3+1)$ Oscillation Parameters}

A 2-flavour model consists of two mass eigenstates and describes the oscillations between these two states. The neutrino oscillations at short-baseline lengths can happen for $\Delta \mathrm{m}^{2} \sim 1 \mathrm{eV}^{2}$ which is many orders larger than the solar and atmospheric mass differences. From the Eq. 1.48 and 1.50, the $(3+1)$ neutrino oscillation probabilities reduces to a 2 -flavour oscillation probabilities. 


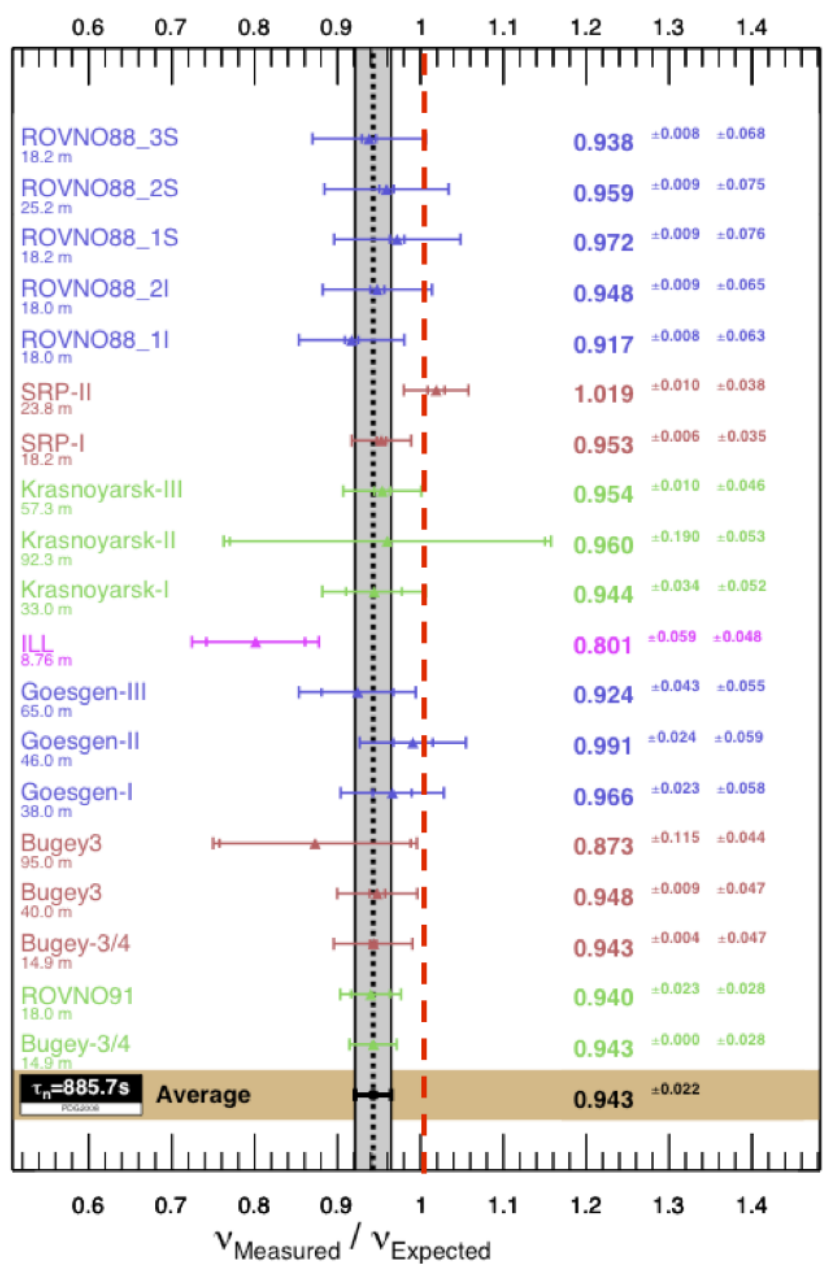

FiguRE 1.15: Shows the ratio between measured and expected neutrino flux from various short-baseline reactor neutrino experimnets. Ref. [86].

Most of the short-baseline experiments have fitted their results with 2-flavour model which has two independent parameters namely mixing angle $\theta$ and mass-squared difference $\Delta \mathrm{m}^{2}$. Very few experiments are done the fit with the $(3+1)$ model. Using the results from reactor experiments, MINOS, IceCube, NEOS, the global fit was performed $[87,88]$. Then, the current best-fit values obtained are shown in Tab. 1.18. The current best-fit values, with one sterile neutrino in the $(3+1)$ model, given by the global fit of all short-baseline experiments, MINOS, IceCube, NEOS experiments (MiniBooNE low energy excess is not included in the fit) is $\Delta \mathrm{m}_{41}^{2} \approx 1.75 \mathrm{eV}^{2}$ and $\sin ^{2} 2 \theta_{\mu e} \approx 1.45 \times 10^{-3}$ . The Tab. 1.17 shows the allowed values for the $(3+1)$ PMNS mixing matrix elements at $1 \sigma, 2 \sigma$ and $3 \sigma$ C.L. 


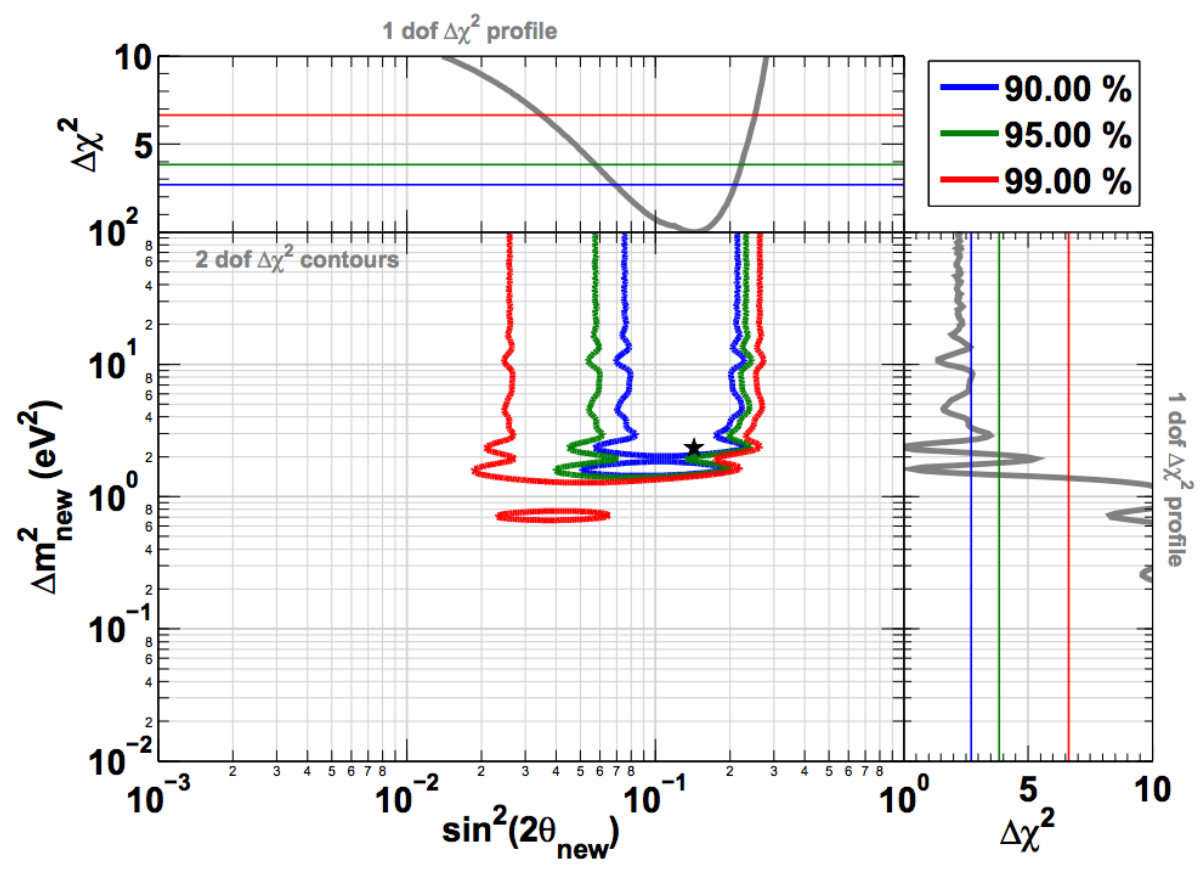

Figure 1.16: Shows the allowed regions in the $\left(\sin ^{2} 2 \theta, \Delta \mathrm{m}^{2}\right)$ plane combining (global fit) all short-baseline reactor neutrino experiments, GALLEX, SAGE and MiniBooNE new results after considering the correlations between these experiments. Ref. [86].

\begin{tabular}{cccc}
$\mathrm{CL}$ & $\left|U_{e 4}\right|^{2}$ & $\left|U_{\mu 4}\right|^{2}$ & $\left|U_{\tau 4}\right|^{2}$ \\
\hline $68.27 \%(1 \sigma)$ & $0.016-0.024$ & $0.011-0.018$ & $\lesssim 0.0032$ \\
$95.45 \%(2 \sigma)$ & $0.013-0.028$ & $0.0083-0.022$ & $\lesssim 0.018$ \\
$99.73 \%(3 \sigma)$ & $0.0098-0.031$ & $0.0060-0.026$ & $\lesssim 0.039$ \\
\hline
\end{tabular}

Figure 1.17: The allowed regions for the $(3+1)$ mixing elements using the PrGlo17 global fit. Ref. [88].

\subsubsection{Searching of a Sterile neutrino with NOvA}

The NOvA experiment as I will describe in the next chapter has a near detector which is placed $\approx 1 \mathrm{~km}$ away from the neutrino source and see a neutrino beam with peak energy at $2 \mathrm{GeV}$, which gives $L / E \approx 1 / 2(\mathrm{~km} / \mathrm{GeV})$. From the previous section, we have shown that NOvA is at a great place to probe the neutrino oscillations caused by active-tosterile mixing with a mass-squared difference, $\Delta \mathrm{m}_{41}^{2} \geq 1 \mathrm{eV}^{2}$. In this thesis, we search for such oscillations by performing the joint-fit between $\nu_{\mu} \rightarrow \nu_{e}$ and $\nu_{\mu} \rightarrow \nu_{\mu}$ channels using data corresponds to $8 \times 10^{20}$ protons on target. We will present our results in the $\left(\sin ^{2} 2 \theta_{\mu e}, \Delta \mathrm{m}_{41}^{2}\right)$ plane by comparing with other previous experimental results. We will also present the constraints on the various $(3+1)$ mixing elements from this analysis. 


\begin{tabular}{|c|c|c|c|c|}
\hline & Glo16A & Glo16B & Glo17 & PrGlo17 \\
\hline$\chi_{\min }^{2}$ & 288.4 & 556.9 & 622.1 & 595.1 \\
\hline $\mathrm{NDF}$ & 250 & 525 & 585 & 579 \\
\hline GoF & $4.8 \%$ & $16 \%$ & $14 \%$ & $31 \%$ \\
\hline$\Delta m_{41}^{2}$ & 1.6 & 1.6 & 1.7 & 1.7 \\
\hline$\left|U_{e 4}\right|^{2}$ & 0.027 & 0.028 & 0.021 & 0.020 \\
\hline$\left|U_{\mu 4}\right|^{2}$ & 0.015 & 0.014 & 0.016 & 0.015 \\
\hline $\sin ^{2} 2 \vartheta_{e \mu}$ & 0.0015 & 0.0015 & 0.0013 & 0.0012 \\
\hline $\sin ^{2} 2 \vartheta_{e e}$ & 0.10 & 0.11 & 0.080 & 0.079 \\
\hline $\sin ^{2} 2 \vartheta_{\mu \mu}$ & 0.058 & 0.054 & 0.062 & 0.058 \\
\hline$\Delta \chi_{\mathrm{NO}}^{2}$ & 53.1 & 51.9 & 51.7 & 47.4 \\
\hline $\mathrm{NDF}_{\mathrm{NO}}$ & 3 & 4 & 4 & 4 \\
\hline$n \sigma_{\mathrm{NO}}$ & 6.7 & 6.4 & 6.4 & 6.1 \\
\hline$\left(\chi_{\min }^{2}\right)_{\mathrm{App}}$ & 94.3 & 94.3 & 94.3 & 77.3 \\
\hline $\mathrm{NDF}_{\mathrm{App}}$ & 84 & 84 & 84 & 78 \\
\hline $\mathrm{GoF}_{\mathrm{App}}$ & $21 \%$ & $21 \%$ & $21 \%$ & $50 \%$ \\
\hline$\Delta m_{41}^{2}$ & 0.61 & 0.61 & 0.61 & 0.97 \\
\hline $\sin ^{2} 2 \vartheta_{e \mu}$ & 0.0058 & 0.0058 & 0.0058 & 0.0026 \\
\hline$\left(\chi_{\min }^{2}\right)_{\text {Dis }}$ & 180.8 & 448.3 & 510.6 & 510.6 \\
\hline $\mathrm{NDF}_{\text {Dis }}$ & 163 & 439 & 499 & 499 \\
\hline $\mathrm{GoF}_{\mathrm{Dis}}$ & $16 \%$ & $37 \%$ & $35 \%$ & $35 \%$ \\
\hline$\Delta m_{41}^{2}$ & 1.7 & 1.7 & 1.7 & 1.7 \\
\hline$\left|U_{e 4}\right|^{2}$ & 0.025 & 0.025 & 0.017 & 0.017 \\
\hline$\left|U_{\mu 4}\right|^{2}$ & 0.011 & 0.0088 & 0.0073 & 0.0073 \\
\hline $\sin ^{2} 2 \vartheta_{e \mu}$ & 0.0011 & 0.00086 & 0.00048 & 0.00048 \\
\hline $\sin ^{2} 2 \vartheta_{e e}$ & 0.097 & 0.097 & 0.065 & 0.065 \\
\hline $\sin ^{2} 2 \vartheta_{\mu \mu}$ & 0.042 & 0.035 & 0.029 & 0.029 \\
\hline$\Delta \chi_{\mathrm{PG}}^{2}$ & 13.4 & 14.4 & 17.2 & 7.2 \\
\hline $\mathrm{NDF}_{\mathrm{PG}}$ & 2 & 2 & 2 & 2 \\
\hline $\mathrm{GoF}_{\mathrm{PG}}$ & $0.13 \%$ & $0.075 \%$ & $0.019 \%$ & $2.7 \%$ \\
\hline
\end{tabular}

FiguRE 1.18: The results from the global fit using the $(3+1)$ model from GLoBES. The last column PrGlo17 gives the current best-fit values which didn't include the MiniBooNE low energy excess into the gloabl fit. Otherwise, all short-baseline reactor experiments, MINOS, IceCube, NEOS results included into the fit. Ref. [88]. 


\section{Chapter 2}

\section{NOvA Experiment}

NOvA (NuMI Off-Axis Electron Neutrino Appearance Experiment) is a long-baseline accelerator-based neutrino oscillation experiment at Fermilab. NOvA has two functionally identical detectors with granular structure. The Near Detector is located 100 meters deep underground, $\sim 1 \mathrm{~km}$ away from the neutrino source at Fermilab and the Far Detector is located on the surface, $810 \mathrm{~km}$ away from the neutrino source at Ash River, Minnesota. Both the detectors are centered $14.6 \mathrm{mrad}$ off-axis to the beam direction as explained in the Section 2.2, and both the detectors use the NuMI (Neutrinos at the Main Injector) neutrino beam. The NOvA Far Detector is located at first maxima of $\nu_{e}$ appearance probability to observe the $\nu_{\mu} \rightarrow \nu_{e}$ oscillations. NOvA main physics goals are mass hierarchy, $\mathrm{CP}$ violation and precision measurement of mixing angle $\theta_{23}$ as I will describe in more detail in Section 2.1.

\subsection{Physics Goals}

The $\nu_{e}$ appearance probability in a 3-flavour model can be written as a sum of atmospheric, solar oscillation probabilities and the interference between these two. The interference term has both Charge-Parity $(\mathrm{CP})$ conserving and violating terms.

$$
P_{\nu_{\mu} \rightarrow \nu_{e}}=P_{\mathrm{atm}}+P_{\mathrm{sol}}+P_{\mathrm{int}}
$$




$$
\begin{aligned}
P_{\mathrm{atm}} & =\sin ^{2} \theta_{23} \sin ^{2} 2 \theta_{13} \sin ^{2}\left(1.27 \frac{\Delta m_{31}^{2} L}{E}\right) \\
P_{\mathrm{sol}} & =\cos ^{2} \theta_{13} \cos ^{2} \theta_{23} \sin ^{2} 2 \theta_{12} \sin ^{2}\left(1.27 \frac{\Delta m_{21}^{2} L}{E}\right) \\
P_{\text {int }} & =J\left[\cos \delta \cos \left(1.27 \frac{\Delta m_{32}^{2} L}{E}\right) \pm \sin \delta \sin \left(1.27 \frac{\Delta m_{32}^{2} L}{E}\right)\right]
\end{aligned}
$$

where $J=\cos \theta_{13} \sin \theta_{12} \sin \theta_{13} \sin \theta_{23} \sin ^{2}\left(1.27 \frac{\Delta m_{31}^{2} L}{E}\right) \sin ^{2}\left(1.27 \frac{\Delta m_{21}^{2} L}{E}\right)$

\subsubsection{Precise Measurement of $\theta_{23}$}

The contribution of the solar term in the Eq. 2.2 is negligible compared to atmospheric term to the appearance probability because the mass ratio is small as shown in Eq. 2.6. Moreover, the atmospheric term is proportional to $\theta_{23}$, which indicates that the NOvA experiment is sensitive to $\theta_{23}$ and can make a measurement of it.

$$
\Delta m_{21}^{2} / \Delta m_{31(32)}^{2} \cong 0.03
$$

\subsubsection{Measurement of $\delta_{C P}$}

The $\mathrm{CP}$ violation could shed light on imbalance between matter and anti-matter in the universe. If $\mathrm{CP}$ is violated, $P\left(\nu_{\alpha} \rightarrow \nu_{\beta}\right) \neq P\left(\nu_{\bar{\alpha}} \rightarrow \nu_{\bar{\beta}}\right)$, for $\alpha \neq \beta$. NOvA is proposed to run in both neutrino mode and anti-neutrino mode and so can measure both these probabilities. As we see from the Eq. 2.2, the interference term in the appearance probability depends on $\mathrm{CP}$ violation phase $\delta_{C P}$. NOvA has the potential to help unravel the mystery by measuring the $\mathrm{CP}$ violating phase.

\subsubsection{Resolving Mass Ordering}

NOvA is placed $810 \mathrm{~km}$ away from the neutrino source and the neutrinos will interact with the Earth's matter while reaching the far detector. Matter effects enhance the $\nu_{\mu} \rightarrow \nu_{e}$ oscillations and suppress the $\bar{\nu}_{\mu} \rightarrow \bar{\nu}_{e}$ oscillations in the normal ordering $\left(\mathrm{m}_{3} i\right.$ $\left.\mathrm{m}_{2} \dot{i} \mathrm{~m}_{1}\right)$ and the effect is reversed in the inverted ordering $\left(\mathrm{m}_{1} \dot{i} \mathrm{~m}_{2} \dot{i} \mathrm{~m}_{3}\right)$. This means NOvA can potentially measure the mass ordering. 


\subsection{NOvA Neutrino Beam}

NOvA uses the existing NuMI facility at Fermilab for its neutrino beam. As shown in Fig. 2.1, making a neutrino beam is a multi-step process. First, we accelerate a proton beam to $120 \mathrm{GeV}$. Second, impinge this $120 \mathrm{GeV}$ proton beam onto a graphite target of approximately 1 meter in length. The secondary mesons produced in this step further decay in flight to give a neutrino. The full details of how we make the beam is given in this Section.

\subsubsection{Making $120 \mathrm{GeV}$ Proton Main Injector Beam}

As you can see in Fig. 2.1, making a proton beam starts with removing an electron from the outer shell of a Hydrogen atom which leaves the $\mathrm{H}^{-}$ions. These ions are accelerated to $400 \mathrm{MeV}$ in a Linac which is then converted to protons in the Booster where they are accelerated to an energy of $8 \mathrm{GeV}$. These $8 \mathrm{GeV}$ protons are made as $1.6 \mu$ s long batches which are sent to Main Injector on a circular accelerator with circumference seven times that of Booster. The Main Injector accelerates the protons to $120 \mathrm{GeV}$. The technique called slip stacking is used to merge these batches on to one another to attain a higher intensity of the proton beam.

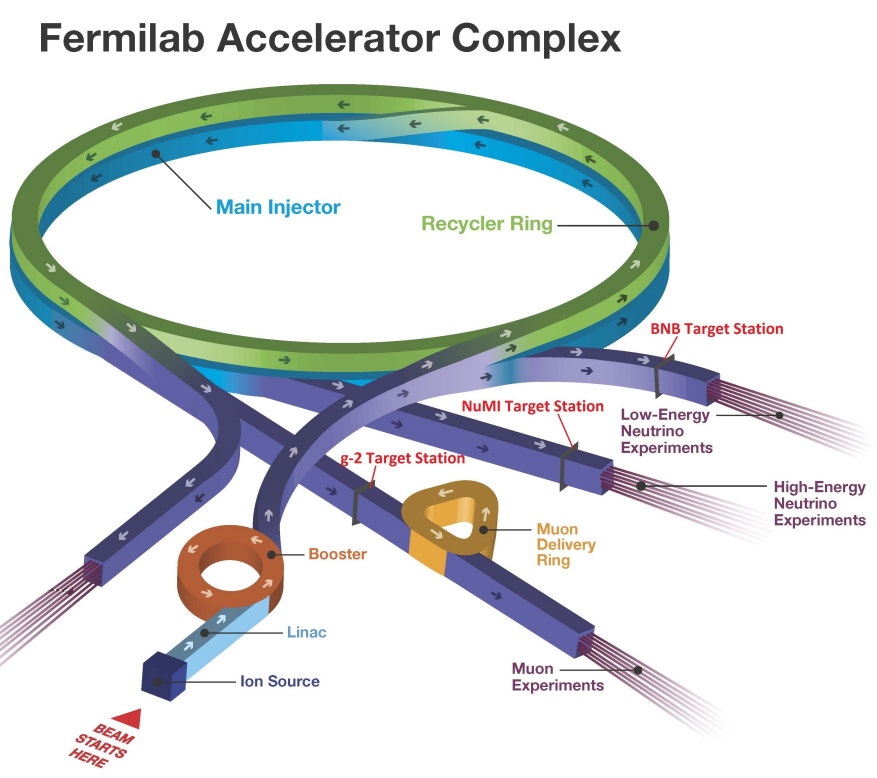

FiguRE 2.1: A cartoon of making neutrinos at Fermilab. 


\subsubsection{Making a Neutrino Beam}

The $120 \mathrm{GeV}$ protons are extracted from the Main Injector and sent down towards the NOvA detectors. The protons are irradiated on to a graphite target of approximately 1 $\mathrm{m}$ length. The carbon-proton interactions produce secondary mesons which are focussed using two magnetic horns towards the decay volume $675 \mathrm{~m}$ long pipe, where they decay in flight to produce the neutrinos, as shown in Fig. 2.2. The magnetic horns polarity can be adjusted to focus any one of the opposite sign hadrons at a time thereby producing either neutrinos or anti-neutrinos.

The NOvA detectors are placed $14.6 \mathrm{mrad}$ off-axis to the neutrino beam direction. The rationale behind this is that in the rest frame of secondary mesons, such as pions and kaons, they decay isotropically producing mono-energetic neutrinos. When these pions and kaons are boosted, the neutrino energy spectrum as seen in the lab frame has a broad distribution, falling off as the angle between the boost direction and neutrino production angle increases. For small angles, the flux and energy of neutrinos produced from the decay in flight of $\pi$ 's as $\pi \rightarrow \mu+\nu$ and intercepted by a detector of an area, $A$ and located at a distance $z$ are given in the lab frame by:

$$
\begin{gathered}
F=\left(\frac{2 \gamma}{1+\gamma^{2} \theta^{2}}\right)^{2} \frac{A}{4 \pi z^{2}} \\
E_{\nu}=\frac{0.43 E_{\pi}}{1+\gamma^{2} \theta^{2}}
\end{gathered}
$$

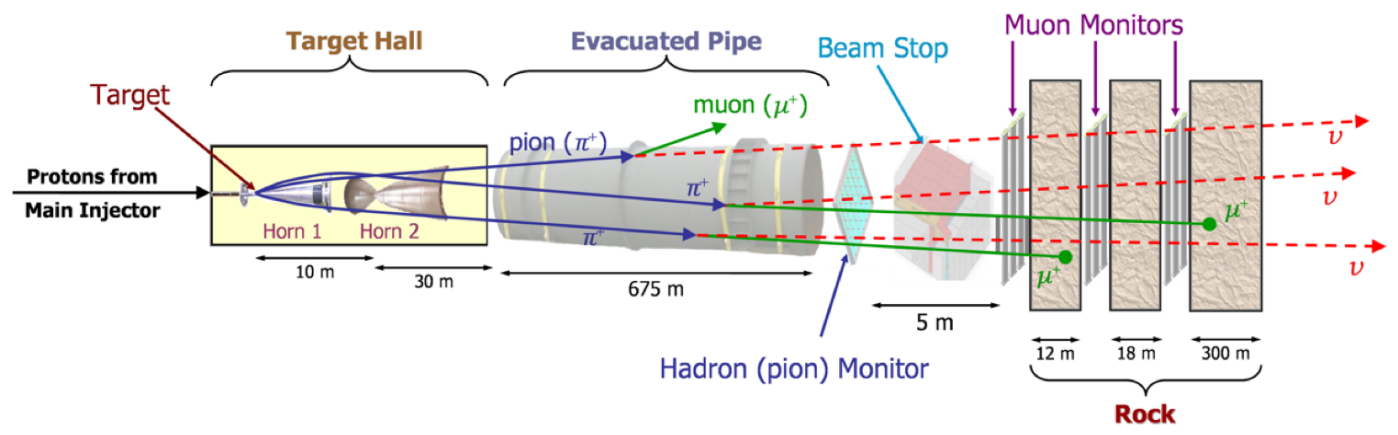

Figure 2.2: A cartoon of the NuMI beamline

The plot on the left in Fig. 2.3 shows the distribution of energy of secondary $\pi$ 's at a various off-axis angle from the beam axis and the plot on the right shows the distribution of energy of neutrinos coming from the decay of secondary mesons in the decay pipe. As we see in Fig. 2.3, the distribution of true neutrino energy of neutrinos, at various off-axis angles, moves to lower energies with a smaller spread as we move away from the 
beam axis. At $14.6 \mathrm{mrad}$ off-axis angle, the NuMI neutrino beam peaks at $2 \mathrm{GeV}$. The beam is mainly $\nu_{\mu}$ are coming from $\pi$ decay and has only a small fraction of $\nu_{e}$ 's mainly coming from K-decay. They have a broader energy spectrum as shown in Fig. 6.5.
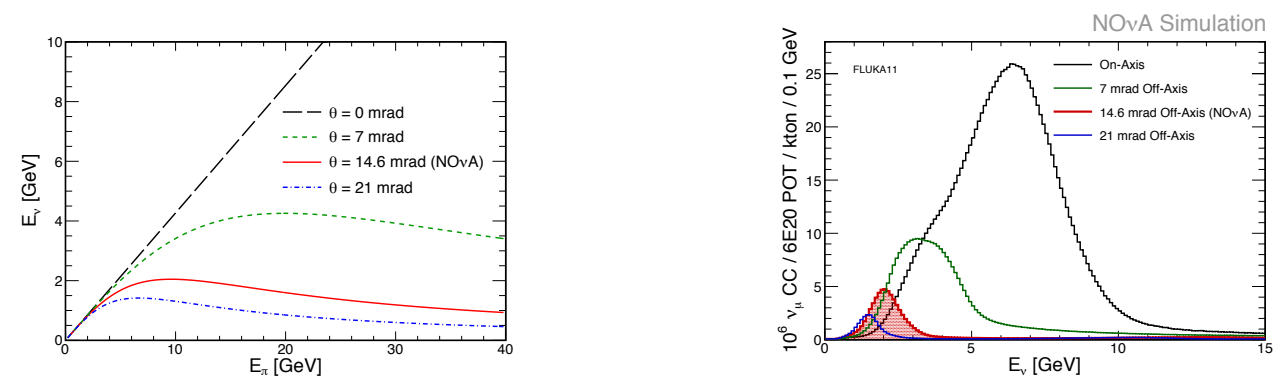

FiguRE 2.3: The distribution of energy for pions (left) and neutrinos (right) at different off-axis angles.

The most common source of muon neutrinos in the NuMI beam are from the decay $\pi^{ \pm} \rightarrow \mu^{ \pm}+\nu_{\mu}\left(\bar{\nu}_{\mu}\right)$ and $\mathrm{K}^{ \pm} \rightarrow \mu^{ \pm}+\nu_{\mu}\left(\bar{\nu}_{\mu}\right)$ because of their highest branching ratio. A summary of main decays is in Table. 2.1.

\begin{tabular}{|c|c|c|}
\hline Decay & Channel & Branching Ratio (\%) \\
\hline 1 & $\pi^{ \pm} \rightarrow \mu^{ \pm}+\nu_{\mu}\left(\bar{\nu}_{\mu}\right)$ & 99.9877 \\
2 & $\pi^{ \pm} \rightarrow e^{ \pm}+\nu_{e}\left(\bar{\nu}_{e}\right)$ & 0.0123 \\
\hline 3 & $\mathrm{~K}^{ \pm} \rightarrow \mu^{ \pm}+\nu_{\mu}\left(\bar{\nu}_{\mu}\right)$ & 63.55 \\
4 & $\mathrm{~K}^{ \pm} \rightarrow \pi^{0}+e^{ \pm}+\nu_{e}\left(\bar{\nu}_{e}\right)$ & 5.07 \\
5 & $\mathrm{~K}^{ \pm} \rightarrow \pi^{0}+\mu^{ \pm}+\nu_{\mu}\left(\bar{\nu}_{\mu}\right)$ & 3.353 \\
\hline 6 & $\mathrm{~K}^{0} \rightarrow \pi^{ \pm}+e^{\mp}+\nu_{e}$ & 40.55 \\
7 & $\mathrm{~K}^{0} \rightarrow \pi^{ \pm}+\mu^{\mp}+\nu_{\mu}$ & 27.04 \\
\hline 8 & $\mu^{ \pm} \rightarrow e^{ \pm}+\nu_{e}\left(\bar{\nu}_{e}\right)+\overline{\nu_{\mu}}\left(\nu_{\mu}\right)$ & 100.0 \\
\hline
\end{tabular}

TABLE 2.1: Most common decay modes and the branching fractions of $\pi \mathrm{s}$, K's and $\mu$ 's to neutrinos.
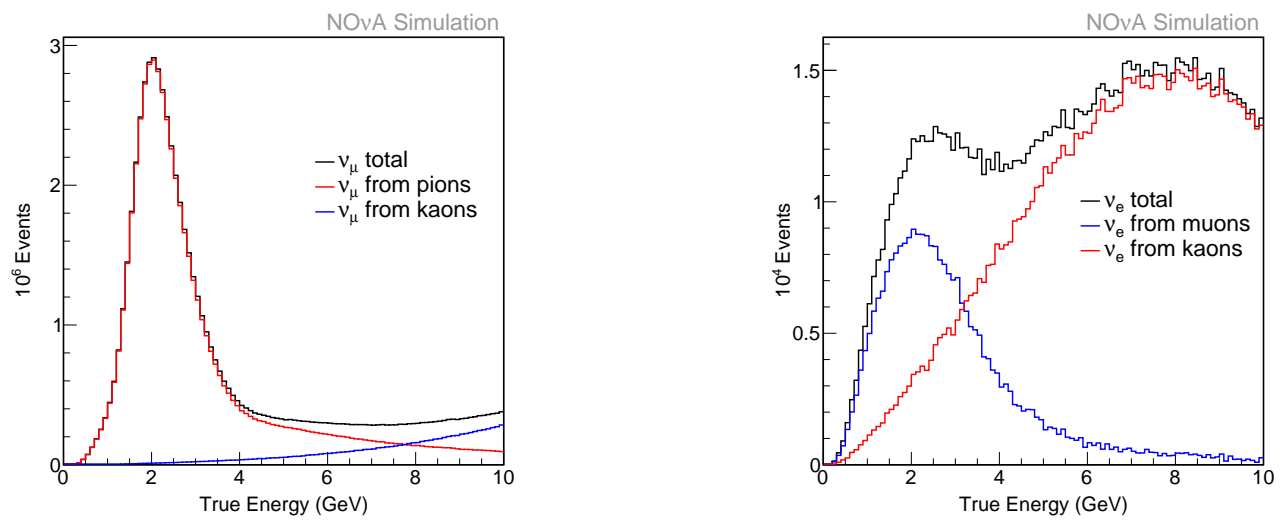

Figure 2.4: The true energy distribution of $\nu_{\mu}$ 's shown in left and of $\nu_{e}$ 's shown in right at the NOvA Near Detector. For $\nu_{\mu}$ 's coming from Kaons are shown in blue, from Pions shown in red, and the total is shown in black. For $\nu_{e}$ 's coming from Kaons are shown in red, from Muons shown in blue, and the total is shown in black. 

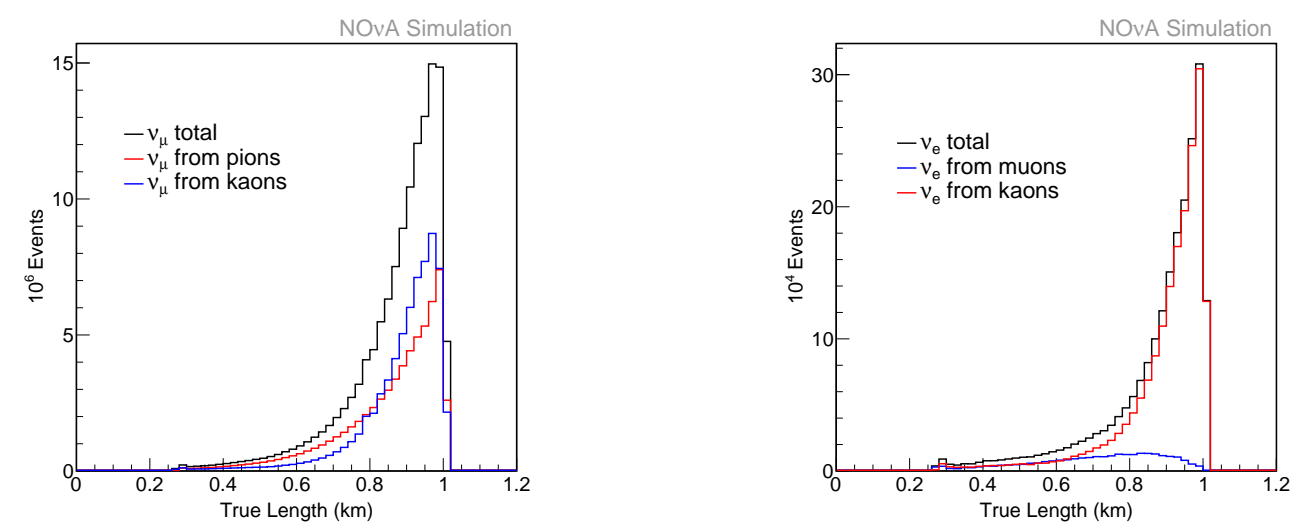

Figure 2.5: The true length distribution of $\nu_{\mu}$ 's shown in left and of $\nu_{e}$ 's shown in right at the NOvA Near Detector. For $\nu_{\mu}$ 's coming from Kaons are shown in blue, from Pions shown in red, and the total is shown in black. For $\nu_{e}$ 's coming from Kaons are shown in red, from Muons shown in blue, and the total is shown in black.
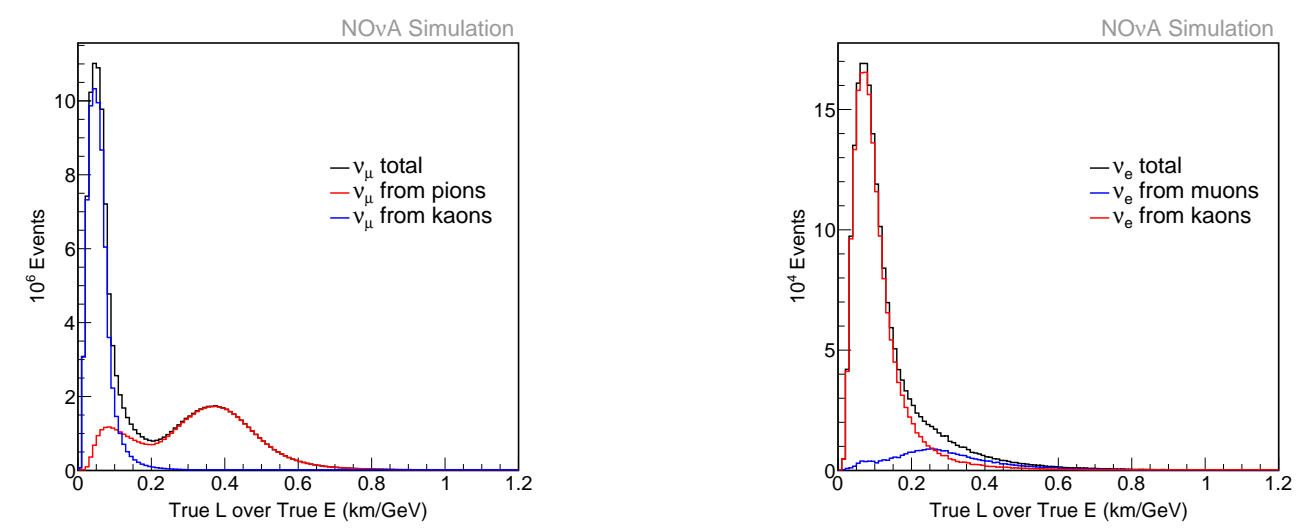

Figure 2.6: The true L over true E energy distribution of $\nu_{\mu}$ 's shown in left and of $\nu_{e}$ 's shown in right at the NOvA Near Detector. For $\nu_{\mu}$ 's coming from Kaons are shown in blue, from Pions shown in red, and the total is shown in black. For $\nu_{e}$ 's coming from Kaons are shown in red, from Muons shown in blue, and the total is shown in black.

The decay of secondary mesons gives both muon and electron neutrinos. The distribution of true energy for both $\nu_{e}$ and $\nu_{\mu}$ is shown in Fig. 6.5. The distribution of distance traveled by neutrinos from decay position of its parents before reaching the Near Detector is shown in Figure. 6.4. Because of the short lifetime, most of the mesons decay at the beginning of the decay pipe. The distribution of true $L / E$ is shown in Fig. 6.2. Neutrino oscillation probabilities are expressed in terms of $L / E$. The Near detector is centered at $L / E \approx 0.4 \mathrm{~km} / \mathrm{GeV}$ and covers a range from 0.3 to 0.5 . The total NuMI flux decomposed by neutrino flavours simulated with FLUKA is shown in Fig. 2.7. 


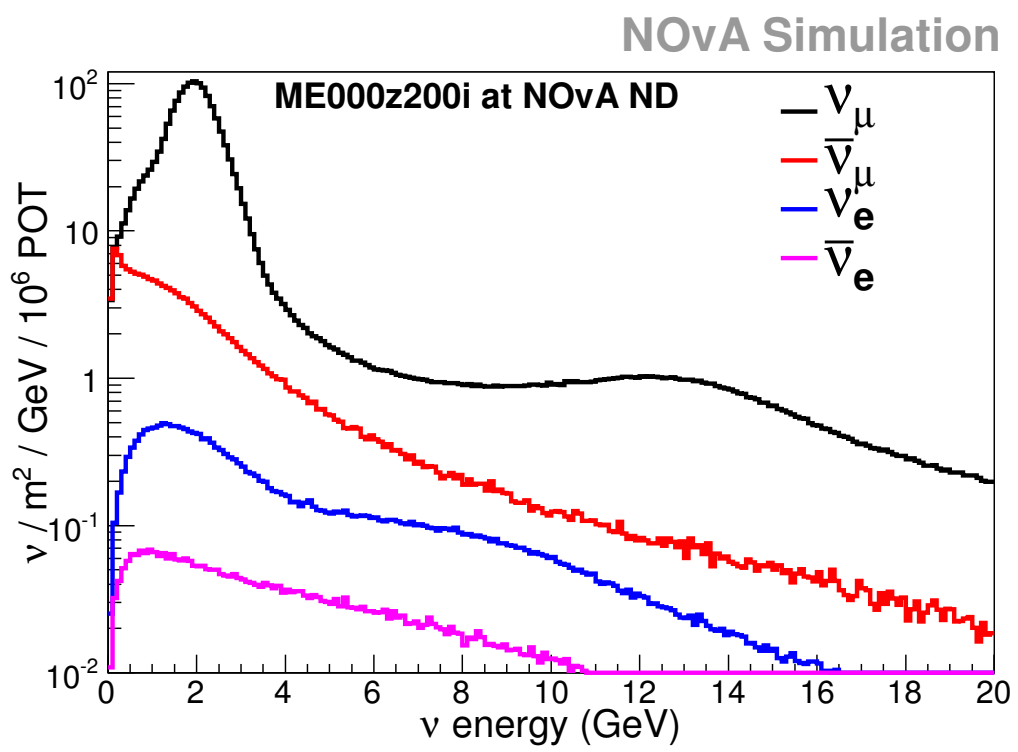

Figure 2.7: NuMI flux at the NOvA Near Detector as simulated with FLUKA showing both $\nu_{e}$ and $\nu_{\mu}$ and anti-particles.

\section{$2.3 \quad$ NOvA Detectors}

The NOvA experiment consists of two functionally identical detectors. The Near Detector is located 330 feet underground about $1 \mathrm{~km}$ away from the neutrino source at Fermilab whereas, the Far Detector is located $810 \mathrm{~km}$ away from Fermilab on the surface at Ash River, Minnesota.

\subsubsection{Two detector Principle}

Most long-baseline neutrino experiments are designed to have at least two detectors, usually, one detector is close to the neutrino source, and other is far away from the source located at the first or second oscillation maxima. The two detectors usually are designed to have identical detector functionality. The advantage of having two identical detectors is that the correlated systematic uncertainties effects between the two will cancel each other. The Near Detector can be used in several ways, to study the neutrino flux which is being sent to the far detector as well as making cross-section studies, etc.

\subsubsection{NOvA Detector Design}

NOvA is a liquid scintillator tracking calorimeter with a modular structure. The basic unit of NOvA detectors is a simple rectangular PVC (Poly-Vinyl Chloride) cell. These 
are glued together to form a module of $32 \mathrm{PVC}$ cells. Every module is equipped with one Avalanche Photo Diode of 32 pixels, explained in Section 2.3.2.4, and one Front-EndBoard (FEB), details in Section 2.3.2.5, as can be seen in Fig. 2.8. These modules are further glued together to form planes and planes are glued to form blocks and finally diblocks. All the FEBs are connected to Data Concentrator Modules (DCM), as explained in Section 2.3.2.6. All the cells are filled with a liquid scintillator, and every cell has a wavelength shifting fiber (WLS) of twice the length of the cell, which will be as explained in Section 2.3.2.3. When the particle travels through the cell, the scintillator emits the scintillation light which is carried away by the fiber to the APD. Then APD converts the light photons to an electrical signal which then digitized by the FEBs and then sent to Data Acquisition (DAQ) System. The DAQ system stores the every light deposit per cell with a time stamp and is used to do the physics analysis.

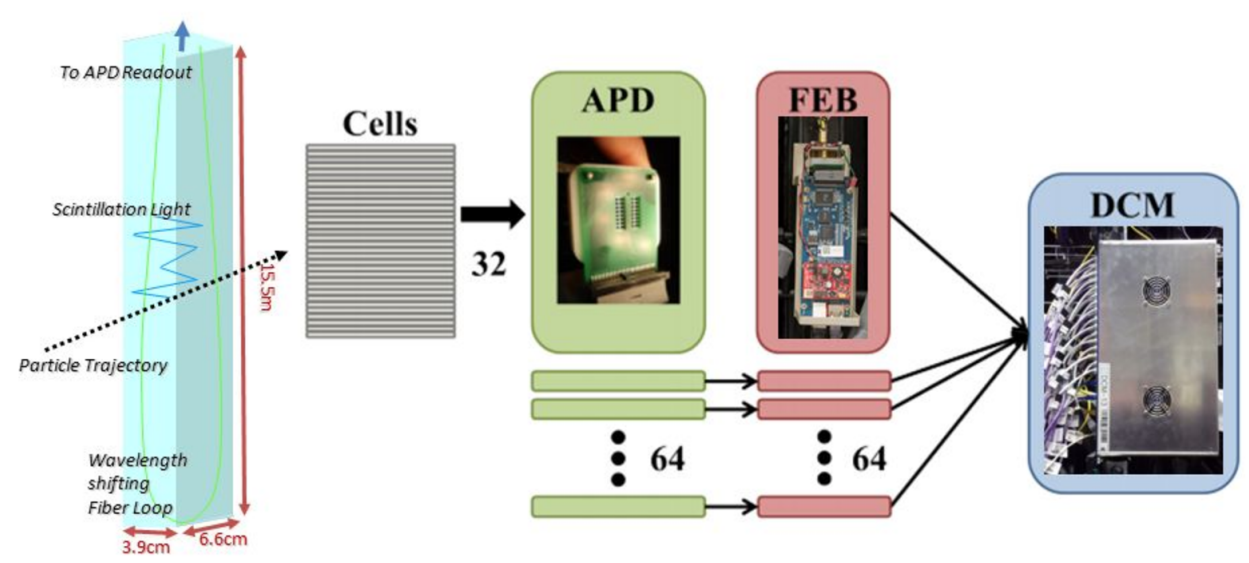

Figure 2.8: NOvA Design Criteria.

\subsubsection{PVC Cell}

PVC cell is a basic NOvA unit in a rectangular shape which has length $L$, width $W$, and breadth $D$. It has $3.8 \mathrm{~cm}$ transverse to the beam direction, $5.9 \mathrm{~cm}$ along the beam and $4 \mathrm{~m}$ length. It is made up of plastic specifically PVC. The walls of a cell are 2 to 4.5 $\mathrm{mm}$ wide, and the inner walls are coated with highly reflective titanium dioxide $\left(\mathrm{TiO}_{2}\right)$ to reflect the photons. The PVC cell is stable to the extent that the liquid scintillator won't affect it for the duration of the experiment. An example of a PVC cell is shown in Fig. 2.9.

\subsubsection{Liquid Scintillator}

The liquid scintillator is the active detector medium and consists nearly $70 \%$ of the detector mass ( $\sim 0.03$ million gallons). The liquid scintillator produces the scintillation 


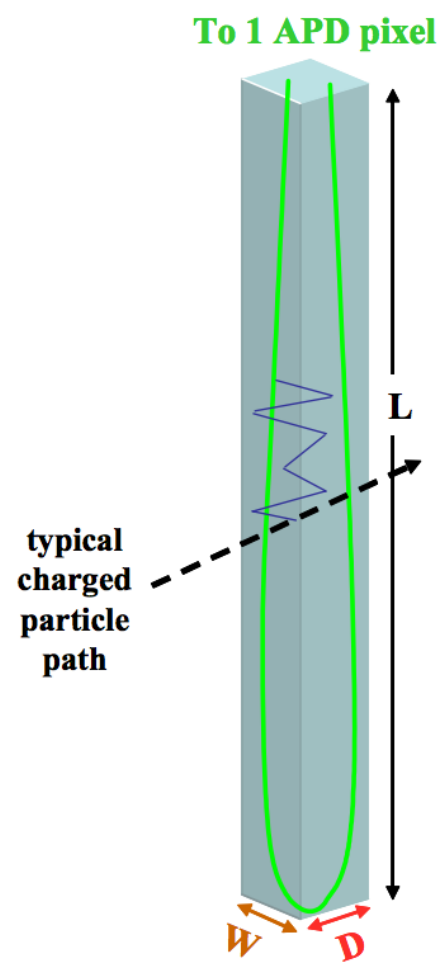

Figure 2.9: The NOvA detector basic unit, single PVC cell.

light when a charged particle passes through it. It is composed of mainly mineral oil, $5 \%$ (by weight) pseudo-cumene (1,2,4-trimethylbenzene) as a scintillant and a chemical additive, PPO (2,5-diphenyloxazole) and bi-MSB (1,4-di(methyl styryl)benzene) are added as wavelength shifters shown in Table. 2.2. Scintillant emits the scintillation light in a wavelength range from 360 to $390 \mathrm{~nm}$, but the added chemical additives, called wavelength shifters, shift the UV wavelengths to a range from 400 to $450 \mathrm{~nm}$ as shown in Fig. 2.10.

\subsubsection{Wavelength Shifting Fiber}

Every PVC cell is equipped with one optical fiber to double the length of a cell, and the fiber has a diameter of $0.7 \mathrm{~mm}$, shown in Fig. 2.9. The fiber is looped at the bottom of the fiber, and both ends of a cell are directed to one pixel of an APD. There is a high concentrated fluorescent dye (K27) with $200 \mathrm{ppm}$ in the fiber to capture the light of wavelength between 490 to $550 \mathrm{~nm}$ wavelength, as shown in Fig. 2.11. This dye improves the capture fraction of light. After the absorption dye emits the light with a wavelength between 450 to $650 \mathrm{~nm}$, it is captured and directed to the APD by the total internal reflection. The optical fiber has a double clad structure with a different refractive index to support the total internal reflection. As the light moves towards the 


\begin{tabular}{|c|c|c|c|c|}
\hline component & purpose & $\begin{array}{c}\text { mass } \\
\text { fraction }\end{array}$ & $\begin{array}{c}\text { volume } \\
\text { (gal) }\end{array}$ & $\begin{array}{c}\text { tot mass } \\
(\mathbf{k g})\end{array}$ \\
\hline mineral oil & solvent & $95.8 \%$ & $3,082,145$ & $9,917,109$ \\
\hline pseudocumene & scintillant & $4.1 \%$ & 128,439 & 425,908 \\
\hline PPO & waveshifter \#1 & $0.091 \%$ & & 9,373 \\
\hline bis-MSB & waveshifter \#2 & $0.0013 \%$ & & 131 \\
\hline Stadis-425 & antistatic agent & $0.0003 \%$ & & 46.6 \\
\hline tocopherol (Vit.E) & antioxidant & $0.0010 \%$ & & 104 \\
\hline Total & & $100.0 \%$ & $3,210,584$ & $10,352,551$ \\
\hline
\end{tabular}

TABLE 2.2: The composition of the liquid scintillator used in NOvA. This table is taken NOvA technical design report.

APD, it gets attenuated up to a factor of ten. The scintillation light is shifted to longer wavelength specifically to red. The shorter wavelengths get more attenuation than the longer wavelengths. Therefore, we need to have an APD with good quantum efficiency for red wavelengths.

\subsubsection{Avalanche Photo Diode}

The primary electronic unit of the NOvA detector is an Avalanche Photo Diode, which is a photodiode that converts photons to an electrical signal. As shown in Fig. 2.12, the APD has 32 pixels, and the two ends of a WLS fiber from every cell in the module of $32 \mathrm{PVC}$ extrusions is connected to one pixel. When the light is absorbed by the pixel, electron-hole pairs are created, and since we apply a high voltage (high electric fields), these electrons are drifted towards the p-n junction where the avalanche process happens. This multiplication of current mainly depends on the amount of electric field at the junction and the mean-free-path of electrons between collisions. The mean-freepath depends on the strength of the accelerating field and the temperature. There could be thermal noise (electron-hole pairs created with the temperature) which mimic the signal. To reduce the current from the thermal noise, the APDs are operated at $-15^{0}$ C. APDs have high quantum efficiency and uniform spectral quantum efficiency which makes APDs more desirable than other photodetectors. The quantum efficiency of an APD is $85 \%$ at the WLS fiber emitted a wavelength of 500 to $550 \mathrm{~nm}$. NOvA APDs operate at a voltage up to a maximum of 425 Volts, at a gain of 100 . This gain can be controlled by the applied bias voltage and the operating temperature. NOvA operates at a gain of 100 or 140 . 

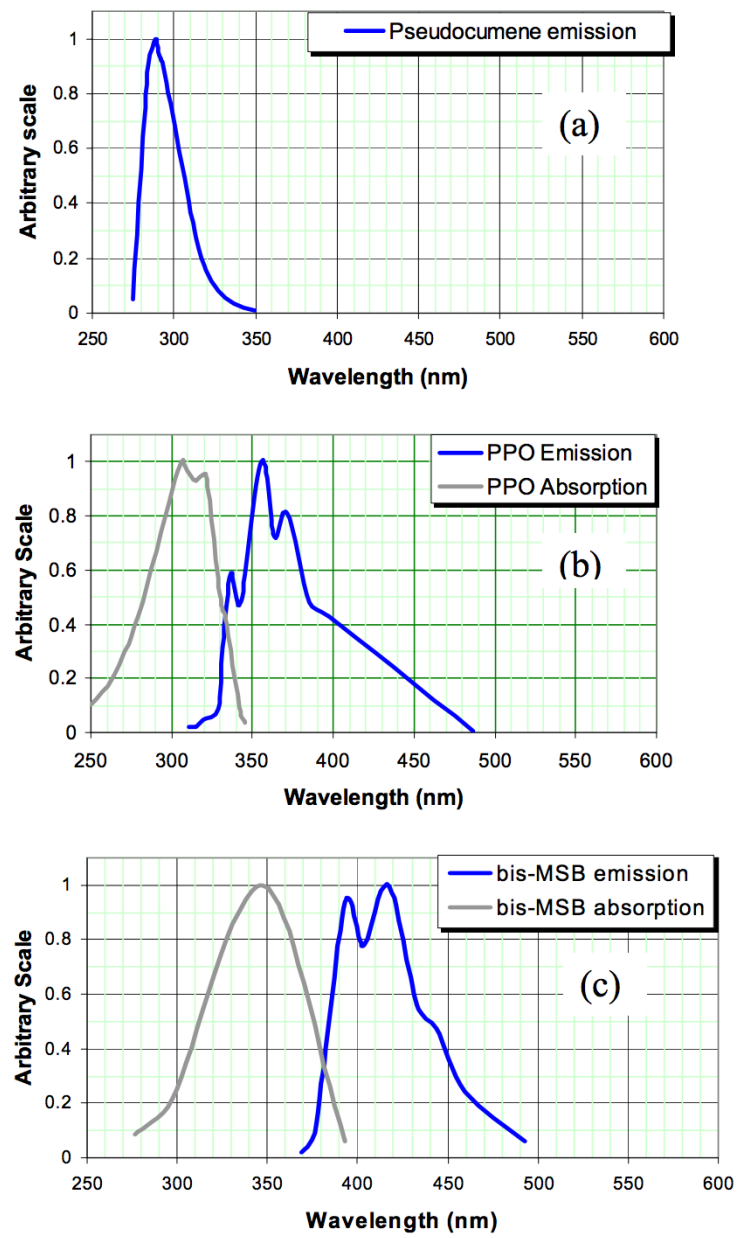

Figure 2.10: Shows how the wavelength shifters shifts the wavelength. This figure is taken NOvA technical design report [89].

APDs are cooled using Thermoelectric (TE) coolers, and each APD is cooled by one TE. The heat from the TE coolers is extracted by circulating the liquid water at temperature $15^{0} \mathrm{C}$. For the Near Detector, we use the heat exchangers to extract the generated heat. Because of heat and cold water, there is a possibility for the dew formation which will effect the APD function. We remove the moisture by circulating dry air across the APD.

\subsubsection{Front-end Electronics Box}

The FEB is connected to APD by a ribbon cable, as shown in Fig. 2.13. The frontend board is a multi-tasking component. It has a Analog to Digital Converter (ADC), Application-Specific Integrated Circuit (ASIC) signal amplifier (provides signal amplification and shaping followed by multiplexing to an array of ADCs), a connector for interfacing to DAQ system to transfer data, the thermoelectric cooler controls (TEC), DAC and ADC for controlling and monitoring, and a Field Programmable Gate Array 


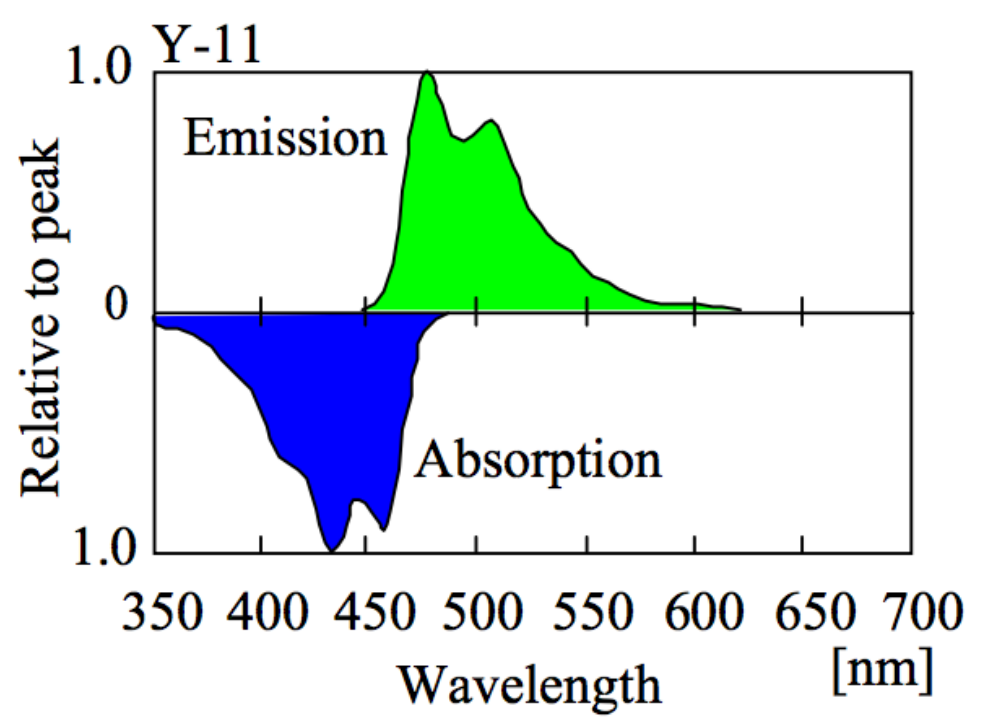

Figure 2.11: The absorption and emission profile of K27 fluorescent dye in the fiber This figure is taken NOvA technical design report [89].
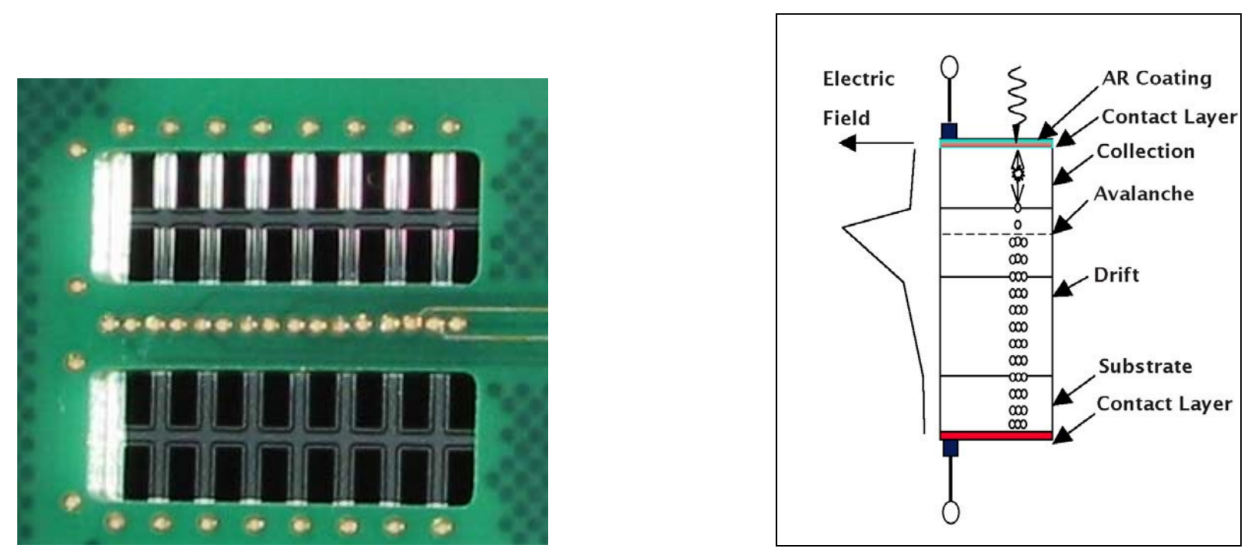

Figure 2.12: A NOvA Avalanche Photo-Diode (left), a diagram of the function of a APD (right).

(FPGA) for digital signal processing and I/O functions and general board monitoring. It requires a voltage of $3.5 \mathrm{~V}$ for maintaining electronic operations, and the TE coolers require $24 \mathrm{~V}$. A example board is shown in Fig. 2.14. The TEC controller circuits are used to maintain a constant APD voltage at a temperature of $-15^{0} \mathrm{C}$. NOvA designed special electronics for the Near Detector because it is exposed to a high rate of neutrino interaction. The sampling rate of the Near Detector is four times that of the Far Detector.

The front-end electronics operated in triggerless mode with no dead time. It is responsible for amplifying and integrating the signal from APD arrays, determining the amplitude and arrival time of the signal and sending the information to the DAQ system. The digitization is performed at a rate of $8 \mathrm{MHz}$ for the Near Detector (higher 


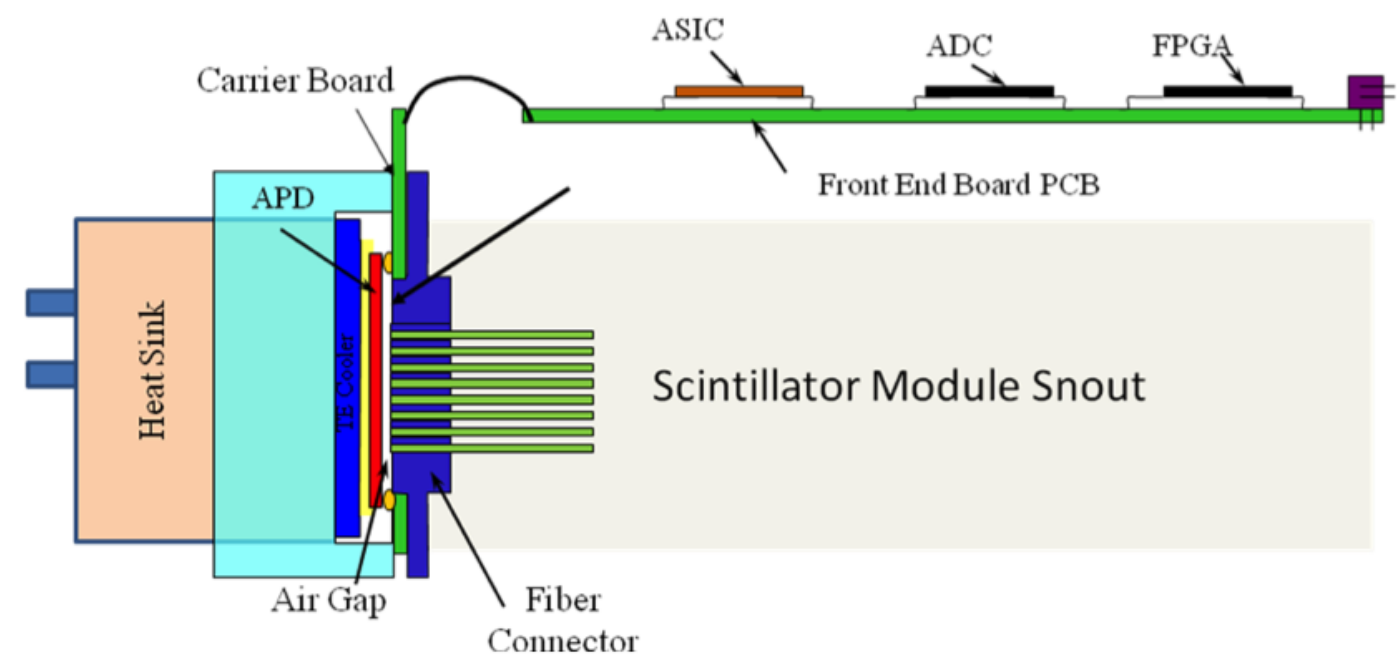

Figure 2.13: Front End Board used in NOvA and it's connections to the APD.

time resolution required due to high interaction rate) and $2 \mathrm{MHz}$ for the Far Detector. The FPGA uses a Digital Signal Processing algorithm to select signal pulses above a programmable threshold for each channel and extract the pulse height and timing edge of the signal. A schematic of FEB is shown in Fig. 2.14.

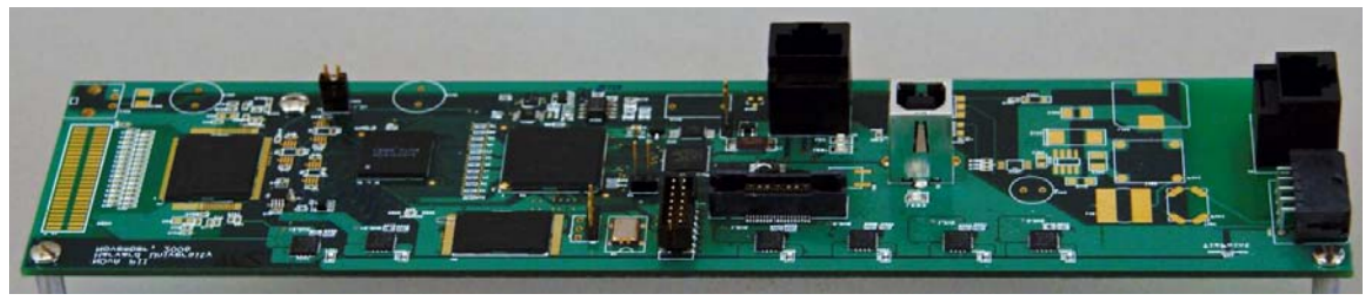

FiguRE 2.14: Layout of the FEB. Major components are the carrier board connector location at the left, which brings the APD signals to the NOvA ASIC, which performs integration, shaping, and multiplexing. The chip immediately to the right is the ADC to digitize the signals, and FPGA for control, signal processing, and communication.

\subsubsection{Data Concentrator Module}

The name itself tells what a Data Concentrator Module (DCM) does, and the block diagram of how it works is shown in Fig. 2.15. The DCM collects, consolidates and concatenates the data from all the $64 \mathrm{FEBs}$; programs configures and monitors the FEBs; and fans out the clock signal and sync command to the FEBs. The DCM has an FPGA which concatenate the hit information from the 64 FEBs and make a data block (a packet) of collected data within a time interval. This data is read by the DCM processor and the application code in the processor packetizes the data into Ethernet packets and transmits these packets to buffer nodes (the data is cached in these nodes) 
through a Gigabit Ethernet port. DCM transmits the data to different buffer nodes through an effective method of round-robin rotation pattern in which each DCM starts with a different node. In this method, no two DCMs transmit the data to the same buffer node, and buffer nodes receive data from only one DCM at a time. The buffer nodes collect data from all DCMs.

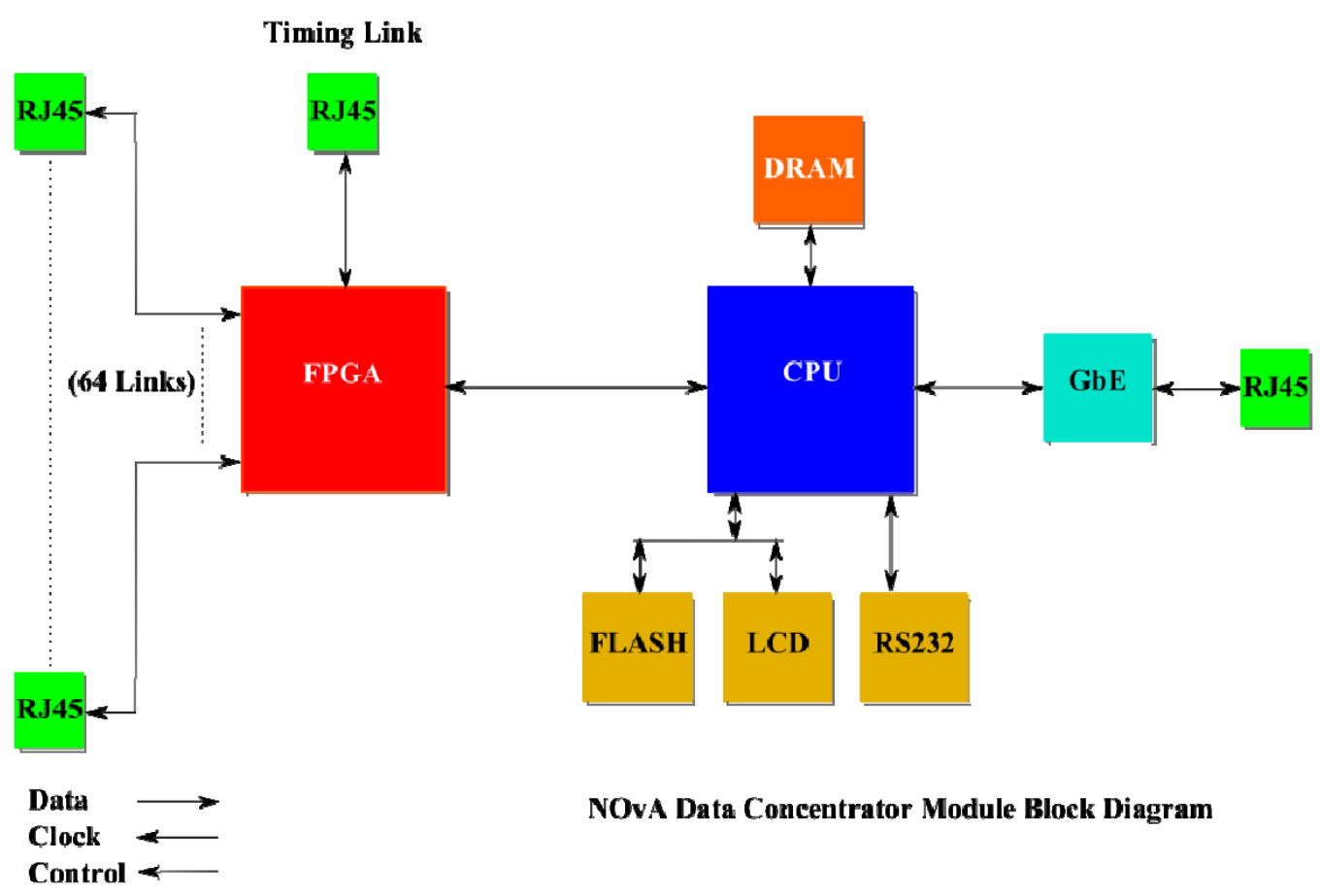

Figure 2.15: Block diagram of a Data Concentrator Module.

\subsection{Data Acquisition System}

Data Acquisition System collects all the data from the APDs into a single stream that be stored and used for the analysis. There is a huge amount of data, all of which is not necessarily is used in physics analysis because all front-end electronics work in non-triggered mode. The collected data is buffered up to 20 seconds until it receives a spill trigger message (a message sent by the spill trigger server) to determine the hits recorded are in or out of spill. The spill is a bunch of neutrinos produced by a bunch of protons hits the C-target. Once the spill signal is received, DAQ keeps the in-spill hits with time stamp in the spill window $30 \mu$ s centered at $11 \mu \mathrm{s}$ based on the timing correlations between the spill and the hits recorded and also keeps the off-spill hits. But the off-spill collected rate, $18 \mathrm{~TB} / \mathrm{yr}$ is much higher than the in-spill collected rate, 190 $\mathrm{GB} / \mathrm{yr}$. When the data is in a cache, there can be many triggers with different trigger 
conditions applied that can be used for calibration and monitoring purpose. A schematic of the DAQ is shown in Fig. 2.16.

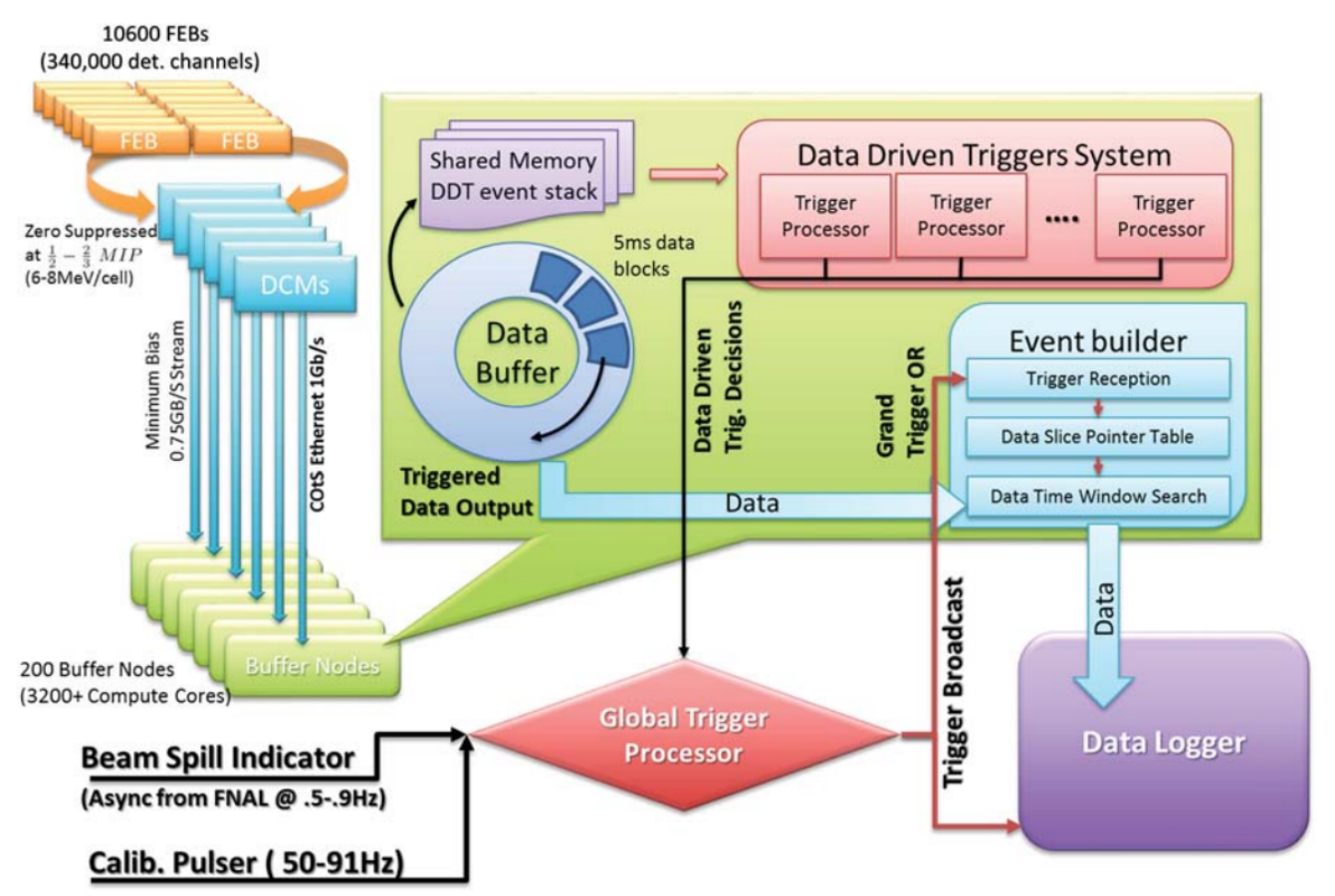

Figure 2.16: Data Acquisition System in NOvA.

\subsubsection{Timing Synchronization}

Since the NOvA detectors have numerous data collecting channels (344,064 at far detector, 20,192 at near detector), it is needed that all the channels are synchronized with each other to ensure the proper event reconstruction. A custom designed timing system is developed for NOvA which contains a master timing distribution unit (MTDU) which is connected to a GPS antenna mounted outside on rooftop as shown in Fig. 2.17. MTDU at Fermilab is also connected to a set of accelerator input lines, and it is connected to a reference pulser system if it is outside Fermilab. NOvA timing system decodes the accelerator signals (at 0.5 to $1.2 \mathrm{~Hz}$ ), and the decoded information is used to activate the NOvA beam spill triggers. MTDU transmits the clock signal to 15 slave TDUs (STDU) by a single mode optical fiber link for far detector. Each STDU is connected to the adjacent STDUs in a daisy-chain fashion with 4 LVDS lines which are Sync, command, clock and sync echo, and each STDU provides the clock signal for 12 DCMs for FD, 6 in X-view and 6 in Y-view. The FEBs are connected to DCM in the same daisy-chain fashion with copper data links which carry the same master clock. The sync echo line is used for implementing "delay learn" for STDUs. The MTDU distributes the clock signal to STDUs which are set in the "learn" mode. When the signal reaches the last STDU in 
the chain, a cable loopback feeds the sync command into the sync echo input which then is propagated along the chain. The STDUs record the time interval between the sync command from MTDU when in learning mode and the input sync command from cable loopback. This time interval is used by STDUs to self-correct for cable propagation delay when STDUs receives the sync command from MTDUs.

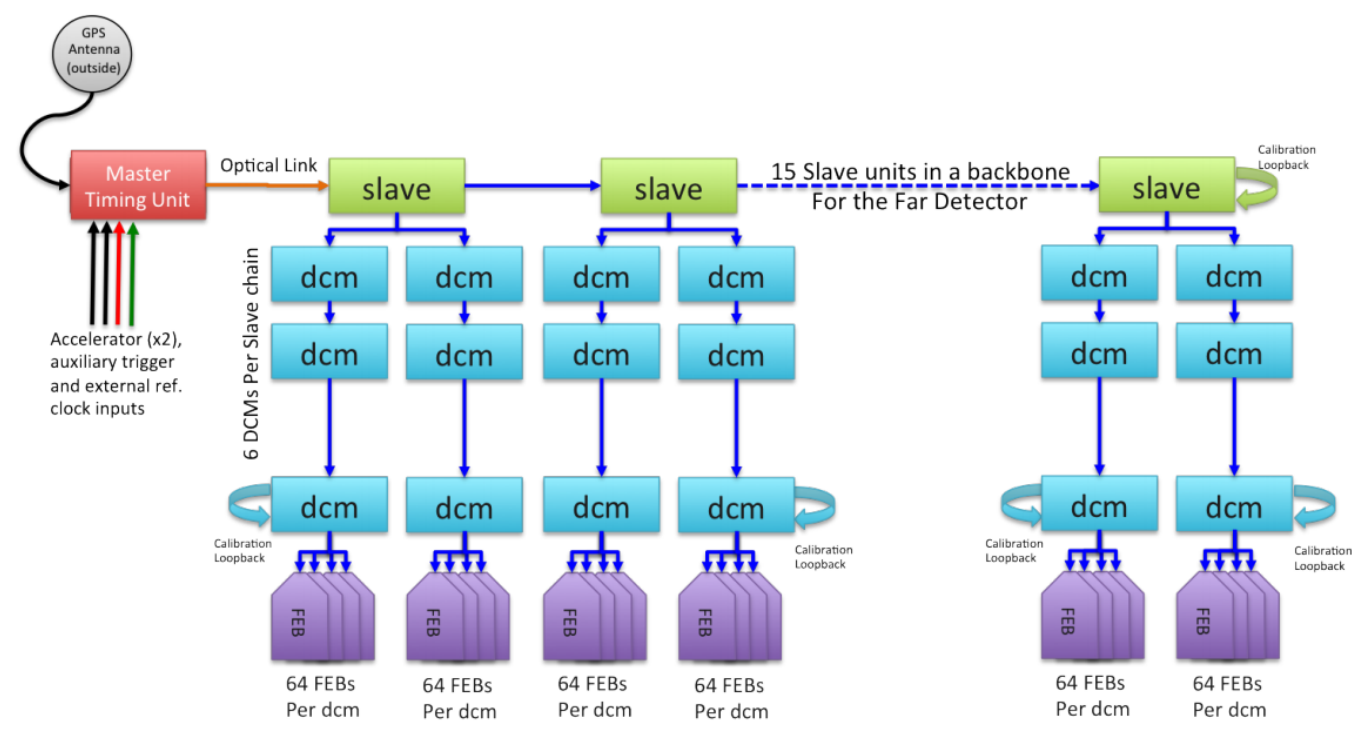

Figure 2.17: Timing distribution system in NOvA.

\subsubsection{Display Visualizer}

The Event Display is the NOvA data visualization package with which one can visualize what an interaction looks like in the NOvA detectors. There are two views in the event display, one is XZ-view or top view, and another is YZ-view or side view corresponding to the alternative horizontal and vertical planes. When a charged particle traverses the detector, it leaves the hits in alternative planes which are used to recreate the 3D tracks or prongs. A hit can be shown either in time or charge; one is according to the hit time in nano seconds, another in according to the charge deposition in units of ADC in the hit. The hit time and charge deposition can be seen in two histograms at the bottom of the event display, time is shown in the bottom left, and a charge is bottom right. Event display also shows the event information which runs and sub run the event belongs to. 


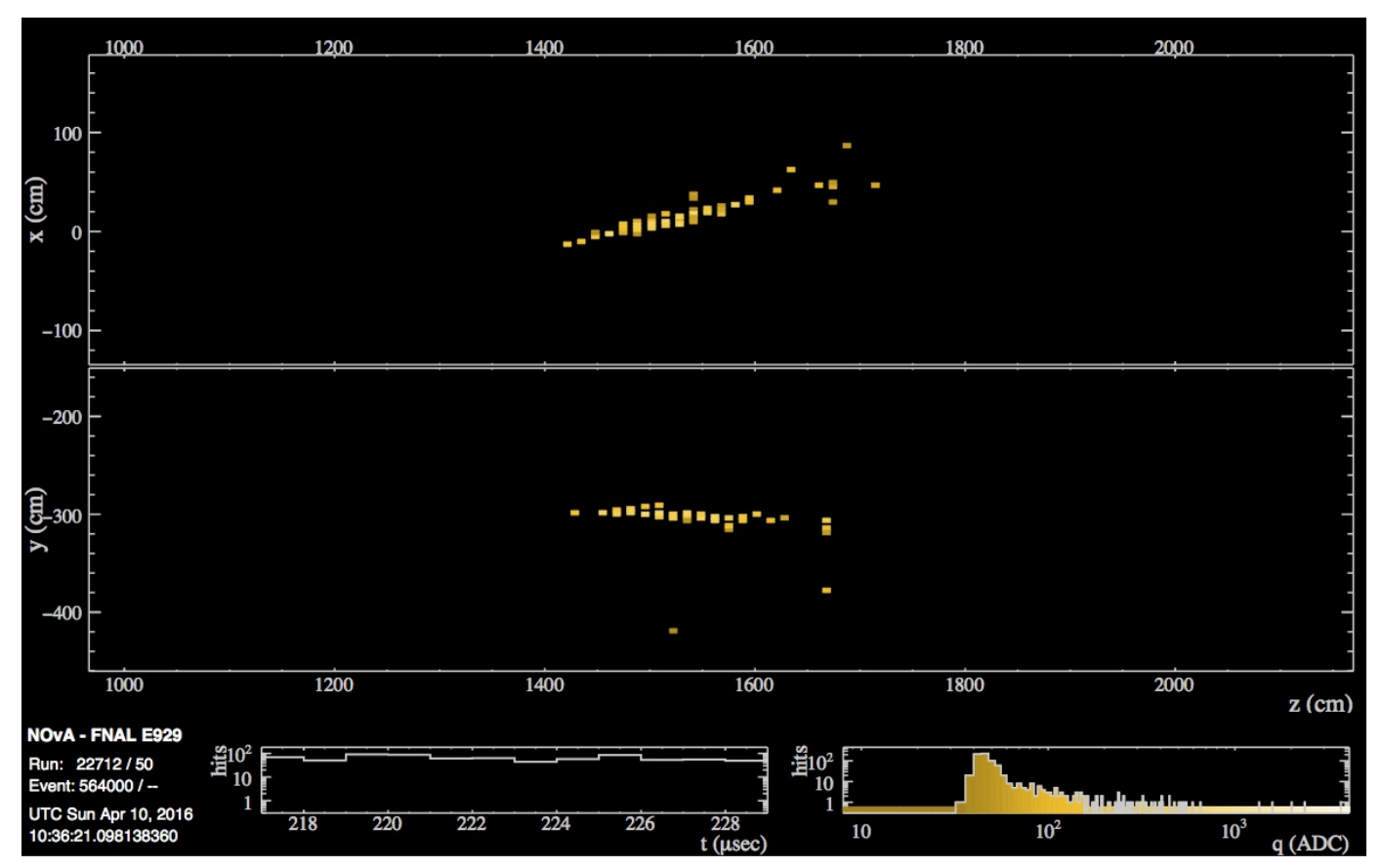

Figure 2.18: This is the event display of the selected appeared $\nu_{e}$ candidate at the Far Detector. 



\section{Chapter 3}

\section{Monte Carlo Simulation}

In this chapter, we describe the simulation used in the NOvA experiment. The simulation starts with simulating the hadron production from $\mathrm{p}-\mathrm{C}$ interactions in the target, the propagation and decay of the secondary mesons, the neutrino interactions in the detector and the interactions of the decay products with the detector material, the light emission, light collection, and the detector response. We will explain these in detail in the following sections.

\subsection{Overview of Monte Carlo Simulation}

Monte Carlo (MC) simulation has a wide range of applications in both science and engineering. Unlike a physical experiment, with MC simulations we can perform numerous experiments on a computer by sampling a random variable. The MC simulation involve three basic steps. The first step is to sample the input random variable from its probability distribution function by generating a random variable from a uniform distribution between 0 and 1 . The second step is to calculate the output variable, and the third step is the analysis of the output. The first step is a two-step process in which the first step involves the generation of random numbers with the uniform distribution between 0 and 1 and, the second step involves the conversion of the uniform variable to a random variable which follows its probability distribution.

There are various MC techniques to transform a uniform variable, between 0 and 1 , to a random variable for example, Inversion Method; Acceptance-Rejection Method; Markov Chain Method and Metropolis-Hastings algorithm, which are explained in Ref. [90]. In the NOvA experiment, neutrino interactions are generated using GENIE (Generates Events for Neutrino Interaction Experiments), event generator [91]. GENIE uses 
the Mersenne Twister [92] random number generator which is implemented in ROOT 's TRandom3 class. In GENIE, every physics process uses it's own random number generator object with independent random number sequence.

In GENIE, the simulation of a neutrino interaction is done as follows,

- Generate a random number between 0 and 1.

- Do kinematic calculations, such as calculating the momentum of the outgoing particles and, calculating scattering angles.

- Calculate the total cross section at a given energy for a given neutrino interaction type and a given target nucleus. This step involves the numerical integration of the corresponding differential cross-section. There are $\sim 10^{2}$ isotopes in a typical detector geometry, $\sim 10^{2}$ interaction modes for a given initial neutrino state, $\sim$ $10^{4}$ differential cross-section evaluations per numerical integration and, so totally $\sim 10^{8}$ differential cross-section evaluations to decide whether a neutrino at a given energy interacts or not. It's a CPU intensive process.

- Sum up the cross section of all physics processes at the given energy.

- Generate an event with the probability $P=\sigma / \sigma_{\max }$, where $\sigma$ is cross-section at a particular energy and $\sigma_{\max }$ is the maximum cross-section at a particular energy.

\subsection{Neutrino Flux}

NOvA uses G4NuMI [93] to simulate the flux, a Geant4 based simulation of the NuMI beamline. G4NuMI uses purely Geant4 physics tools for the beam simulation. Geant4 version 4.9.2p03 with FTFP_BERT [93] hadronic physics list is used. FTFP_BERT model is a combination of FRITIOF Precompound model [94] which is based on the FRITIOF description of string excitation and fragmentation and Bertini Cascade Model [95] which is Geant4 based Bertini cascade for primary protons, neutrons, pions and kaons below $10 \mathrm{GeV}$. The output of the G4NuMI consists of ntuples, a ROOT tree with each variable as a branch, of the neutrino parent decays, and which parent is giving which kind of neutrino interaction and the kinematic variables.

A simulation of a variety of physics processes with a wide range of energies from $\mathrm{eV}$ to $\mathrm{TeV}$ is only possible by combining different models which are valid for specific particles and at a certain energy range. The resulting flux files contain the simulated neutrinos along with their parentage information. 


\subsection{Particle Propagation}

Geant4 provides the information about the secondary particles coming out of the primary p-C interactions, the secondary and tertiary interactions of secondary mesons within the target and, propagating these secondary mesons through magnetic horns and decay pipes, and the decay of secondary mesons which produce the neutrinos.

Geant4 is used for propagating particles through the detector geometry which come out of the physics generators such as GENIE. The particles propagate through matter and interact according to Geant4 physics lists such as FTFP, BERT. It considers various processes allowed by the particles for example energy loss, hadronic and electromagnetic interactions, multiple scattering and decay. The energy loss by a particle leaves a detectable signal in the active volume of the detector which must be recorded. In NOvA, this information is recorded into a $\mathrm{C}++$ object called FLSHit (Fiber in a Liquid Scintillator Hit).

\subsection{Neutrino Interactions}

The neutrino interaction processes with the detector material are simulated using GENIE. GENIE uses the neutrino flux from G4NuMI combining with pre-calculated neutrino cross-sections to generate the various types of neutrino-induced interactions in the detector, specifically determining the energies of the neutrinos which will interact. Depending on the total cross-section of the individual interaction type, GENIE generates the corresponding neutrino interaction. The kinematics of the resultant particles from a particular interaction are determined from that particular differential cross-section. GENIE uses a modified Relativistic Fermi Gas model to simulate the neutrino-nucleus interactions. The interactions of final state hadrons with the nucleons within the nucleus are simulated using INTRANUKE package [91] and which depends on the density of the nucleons and hadron cross-sections. These interactions are called Final State Interactions (FSI). NOvA also simulates the cosmic ray interactions using the Cosmic RaY (CRY) generator [96] with a wide range of energies.

The resulting particles from the neutrino interaction generator are then propagated through the detector geometry by Geant4 using QGSP_BERT_HP physics list. This is a combination of three basic physics lists to describe the whole variety of interactions. QGSP (Quark Gluon String Precompound) physics list model the high energy interactions of hadrons (such as protons, neutrons, pions, and kaons) and nuclei with the quark-gluon string model, and pre-compound model to model the de-excitation of the nucleus, BERT includes the Geant4 Bertini cascade for primary hadrons below $\sim 10$ 
$\mathrm{GeV}$, HP consists of data-driven neutron package with High Precision to transport neutrons with energies below $20 \mathrm{MeV}$. The combination of these three physics lists results in better agreement with the data than other models.

\subsection{Photon Transport}

Even though Geant 4 can simulate the optical photon processes, it's very time-consuming. NOvA uses a custom designed ray tracing simulation which uses the scintillation spectrum of the liquid scintillator, the measured reflectivity of the PVC cell walls and the absorption spectrum of WLS fiber. Fig. 3.1 shows the resulting scintillation photon collection rate as a function of the distance between the photon emission and absorption, and the time difference between photon emission and photon absorption.

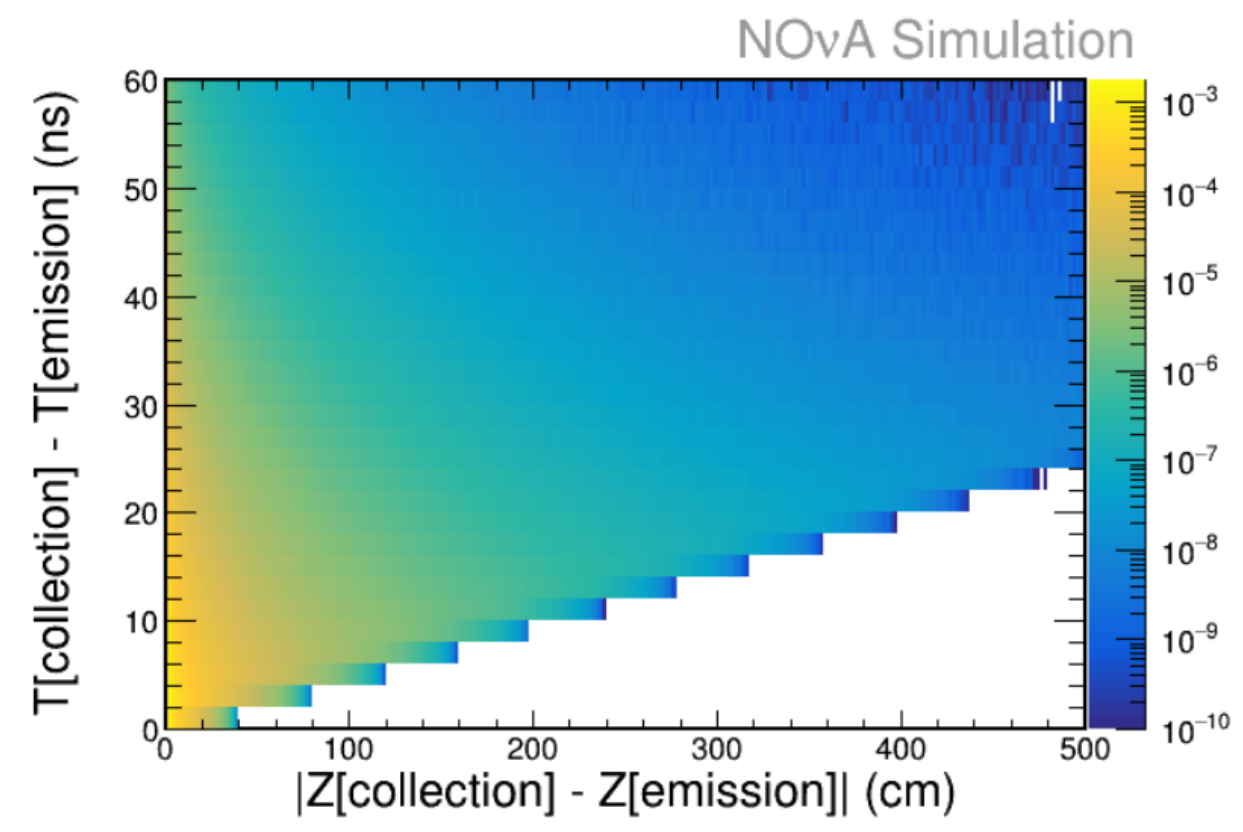

Figure 3.1: The light collection rate by WLS fiber in NOvA using the ray tracing simulation.

The light collected by the WLS fiber is then transported along each half of the fiber to the APD. We can obtain the light collected by an APD from all $\Delta \mathrm{Z}$ (the distance between photon emission and absorption along the detector longitudinal axis, Z-axis) as a function of time for a given energy deposit. The number of survival photons can be estimated from the quality control data testing the optical fiber collected during the construction of the detector. The travel speed of photons can also be calculated using the fiber ray tracing simulation as travel time up the fiber divided by the distance traveled. 


\subsection{Electronic Simulation}

This step in the simulation consists of a conversion from the photo-electrons collected by the APD to a digital signal. The front-end electronics namely ASIC, a Capacitor:Resistor

- Resistor:Capacitor (CR-RC) circuit, shapes the analog signal from the APDs. The response of the CR-RC circuit is given by Eq. 3.1 as

$$
f(t)=N_{p e} \frac{F}{F-R}\left(e^{-\left(t-t_{0}\right) / F}-e^{-\left(t-t_{0}\right) / R}\right)
$$

where $F$ and $R$ are the fall time and rise time of CR-RC circuit, $t_{0}$ is the collection time of the photo electron pulse and $N_{p e}$ is the number of photo-electrons.

We also simulate the effect of voltage and current noise which distorts the shaped pulse. The shaped pulse is then converted from photo electron units to ADC using a conversion factor from the charge injection studies. The baseline is added obtained from the pedestal scan. A pedestal scan is a baseline scan over electronics to determine the electronic noise when there is no NuMI signal. Finally, an FPGA register the hits if the ADC value $A D C_{i}-A D C_{i-3}$ is above the threshold, where $A D C_{i}$ is ADC value at the $i^{t h}$ time slice and $A D C_{i-3}$ is $\mathrm{ADC}$ value at the $(i-3)^{t h}$ time slice. The threshold is set by performing the pedestal scan over all the channels in the real data taking, and in simulation, the threshold is set from the distribution of real-time thresholds. In the cells with no physics hits, noise hits are taken from real-time detector data, but for the near detector, these noise hits are taken from pedestal scan.

\subsection{Monte Carlo Simulation Tuning}

The Monte Carlo Simulation is tuned to raw detector data using the data taken outside the beam spill window. Low-level variables can be compared between data and MC simulation, such as photo-electron spectrum as shown in Fig. 3.2. The MC simulation is tuned to data for the overall light level in photon transport stage and for the noise coefficients in the digitization stage. The data and MC simulation agree well near the peak region, but the saturation modeling in MC simulation doesn't match the data.

Possible reasons that this doesn't match include that I will describe below. We could be missing something in our MC for example APD sag, Scintillator quenching and Diblock masking. In an APD sag, when one pixel has an energy deposit, the output of other channels is reduced by $1.86 \%$ as seen in the charge injection studies. When all channels are triggered, the baseline of all channels is reduced by $1.86 \%$ of the total 

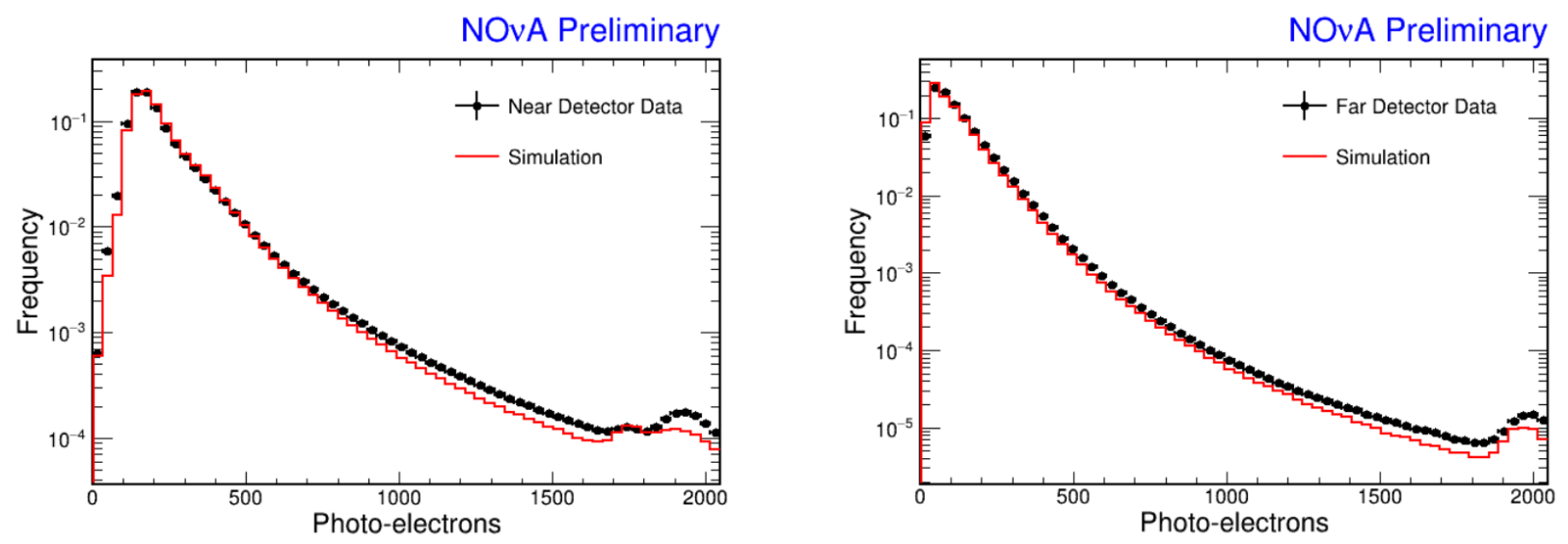

FiguRE 3.2: The data amd MC simulation comparison for the number of photoelectrons for both ND (left) and FD (right). As we see, both agree well in the peak region.

charge deposited in the APD as shown in Fig. 3.3. When an APD sag happens, if the $A D C_{i}-A D C_{i-3}$ is larger than the channel threshold, the DSP algorithm considers it as a signal. APD Sag usually happens at FD if the charge deposition is 250 times FD threshold, 700 times than ND threshold. The benchtop charge injection studies shown that sag happens after few microseconds after the actual hit time so that it can be easily removed with the timing cuts.

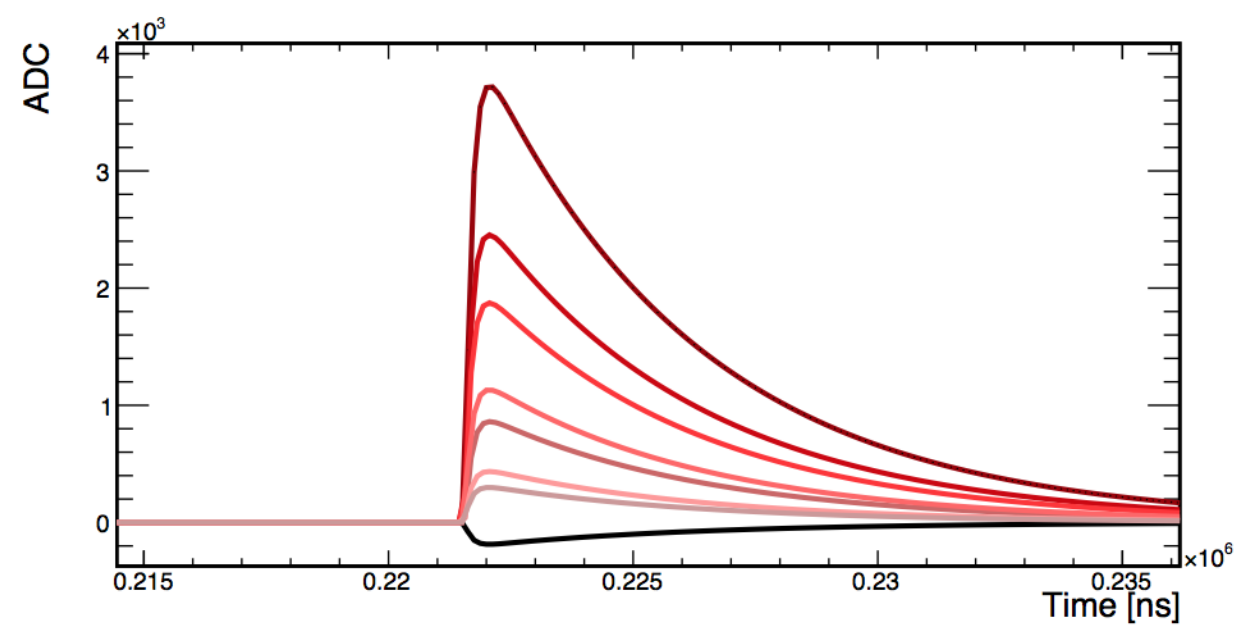

Figure 3.3: APD sag as seen in a single APD. These are the simulated readout traces. The readout trace shown in black is for channel which had no energy deposit, but exhibitibg APD sag due to red traces of other channels. Refer [97].

The second possible effect is scintillator quenching. When the detector is hit by high energetic particles, the energy deposition can be high enough that the scintillation light to quench. In general, the scintillation light is proportional to the energy deposited, but it has non-linear behavior at high energy deposits. The quenching effect in the organic 
scintillators was studied by John B. Birks [98] and described with an empirical equation. The light yield per path length at high energy loss rates is given by Birks' law as

$$
\frac{d L}{d x}=\frac{\frac{\mathrm{dE}}{\mathrm{dx}}}{1+\mathrm{k}_{\mathrm{B}} \frac{\mathrm{dE}}{\mathrm{dx}}} ; \quad \text { where } \mathrm{k}_{B} \text { is the Birks constant. }
$$

The second order correction to the light yield per path length was done by C.N. Chou [99]. The constants $\mathrm{k}_{B}$ and $\mathrm{k}_{C}$ are measured to be $0.01 \mathrm{~g} / \mathrm{cm}^{2} / \mathrm{MeV}$ and $0.0 \mathrm{~g} / \mathrm{cm}^{2} / \mathrm{MeV}$ respectively [], which leads to the better data MC agreement in NOvA.

$$
\frac{d L}{d x}=\frac{\frac{\mathrm{dE}}{\mathrm{dx}}}{1+\mathrm{k}_{\mathrm{B}} \frac{\mathrm{dE}}{\mathrm{dx}}+\mathrm{k}_{\mathrm{C}}\left(\frac{\mathrm{dE}}{\mathrm{dx}}\right)^{2}} ; \quad \text { where } \mathrm{k}_{C} \text { is the Chou constant. }
$$

In Di-block masking, we drop out few readout channels or sometimes a full diblock from data taking that potentially.

\subsection{Cherenkov Light}

At the given wavelength and per unit distance, the number of photons produced from Cherenkov radiation is given by

$$
\frac{d^{2} N_{\gamma}}{d x d \lambda}=\frac{2 \pi \alpha}{\lambda^{2}}\left(1-\frac{1}{\beta^{2} n^{2}}\right)
$$

where $\alpha=1 / 137$ is a fine structure constant, $\mathrm{n}$ is refractive index of the medium. The number of Cherenkov photons emitted at various $\beta$ as a function of wavelength $\lambda$ is shown in Fig. 3.4. As can be seen Fig. 3.4, the number of photons emitted at a wavelength ranging from 400 to $500 \mathrm{~nm}$, which is the favorable absorption range of dye k-27 in NOvA wavelength shifting fiber, is low. But, more number of photons emitted with a wavelength from 200 to $400 \mathrm{~nm}$ which can be absorbed immediately after they produce as seen from Fig. 3.5. This makes the distinction between Cherenkov light and scintillation light difficult. The number of Cherenkov photons emitted by electrons, muons and protons is shown in Fig. 3.6. Higher the value of $\beta$, more the number of Cherenkov of photons emitted by the particles.

For the better calibration procedure, a combination of scintillation light and Cherenkov light must be used. Since muons are having higher $\beta$, a combination of scintillation light and Cherenkov light is used in the calibration procedure for absolute energy scale 


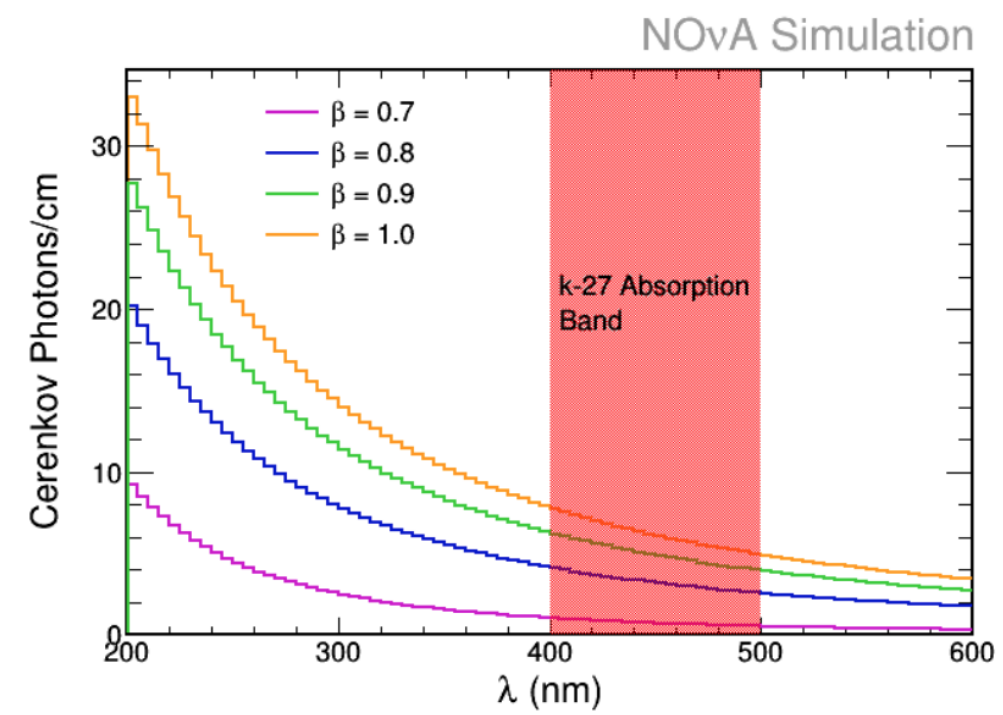

FIGURE 3.4: Shows the number of Cherenkov photons emitted as a function of wavelength at different energy parameter $\beta$. The red shaded band is the abosrption range of k-27 coated on the walls of PVC cell. Refer [101].

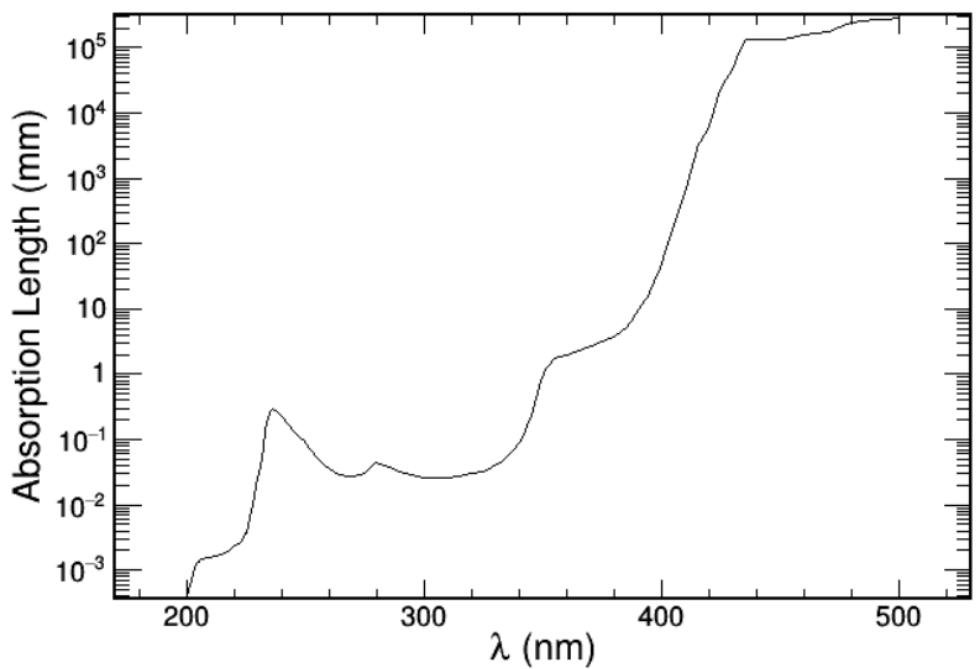

FiguRE 3.5: Shows the absorption length with the wavelength for the NOvA scintillator. Refer [101]

measurement. Even for hadronic system, we must consider both, otherwise they will be mis-calibrated.

In summary, the NOvA simulation does well to simulate the real detector working conditions at every stage. Any difference between the data and MC is treated as a systematic uncertainty. 


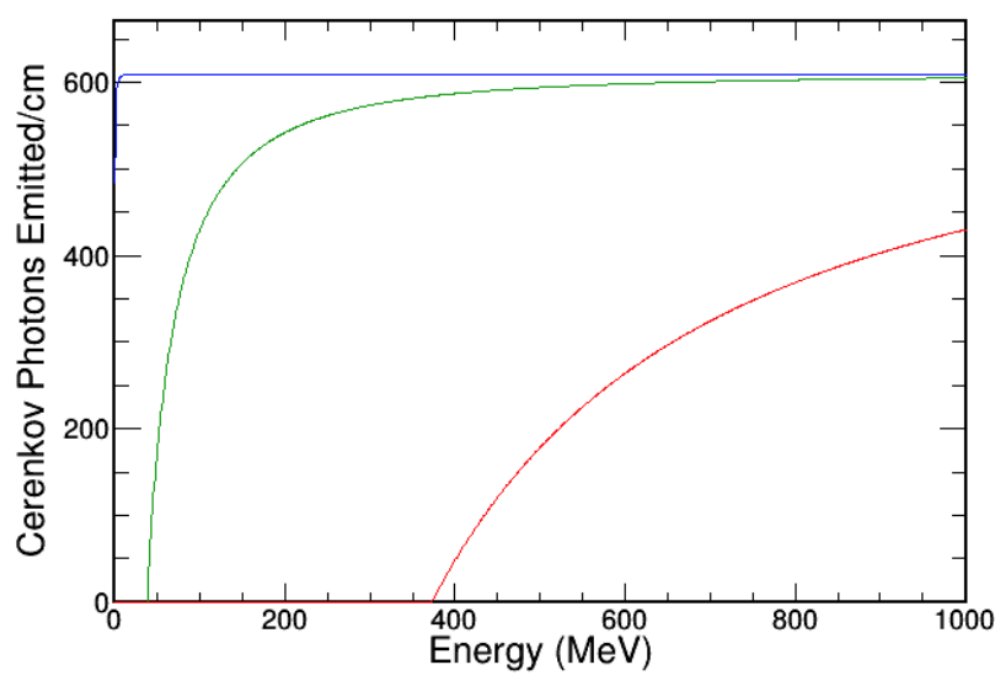

FiguRE 3.6: Shows the number of Cherenkov photons emitted with energy for muons (green), electron (blue) and protons (red). Refer [101]. 



\section{Chapter 4}

\section{Event Reconstruction and Paticle Identification}

This chapter details the calibration of NOvA detectors in Section 4.1, energy reconstruction of neutrino interactions in Section 4.4, object reconstruction in Section 4.2 and the particle identification in Section 4.3. In the event reconstruction, we make the data products which are c++ objects such as vertices, tracks, and prongs, etc. using the list of cells with energy deposits. These products are later used for the analysis.

\subsection{Calibration}

The purpose of calibration is to ensure a uniform detector response across the detector. NOvA detectors are fine-grained equipped with alternating $\mathrm{X}$ and $\mathrm{Y}$ planes of extruded PVC cells. Each cell has a Wavelength Shifting Fiber (WLS) to transport the deposited photons to the APD. APD converts these photons to an analog signal. The ADC on the FEB digitizes the analog signal. In general, the ADC response is linearly dependent on the number of photo-electrons. We need two different types of calibration, one is attenuation calibration, to correct for photons lost along the length of the WLS, and absolute calibration to correct the photo-electrons to an amount of deposited energy. These are explained in Section 4.1.1 and absolute calibration in Section 4.1.2.

\subsubsection{Attenuation Calibration}

Cosmic ray muons are used for the attenuation calibration which is explained in Sec. 4.1.1. The reconstructed muon track is used for this purpose. We use the tricell hits for 
calibrating the NOvA detectors. A tricell is a hit where, say for $\mathrm{Y}$ view, we consider the hits that enter through upper wall and exit through the lower wall of a cell, so it may strike the neighboring cells as shown in Fig. 4.1. This method ensures that the distinction between a signal hit and noise hit because there is a well-defined path length through the cell for tricell hits.

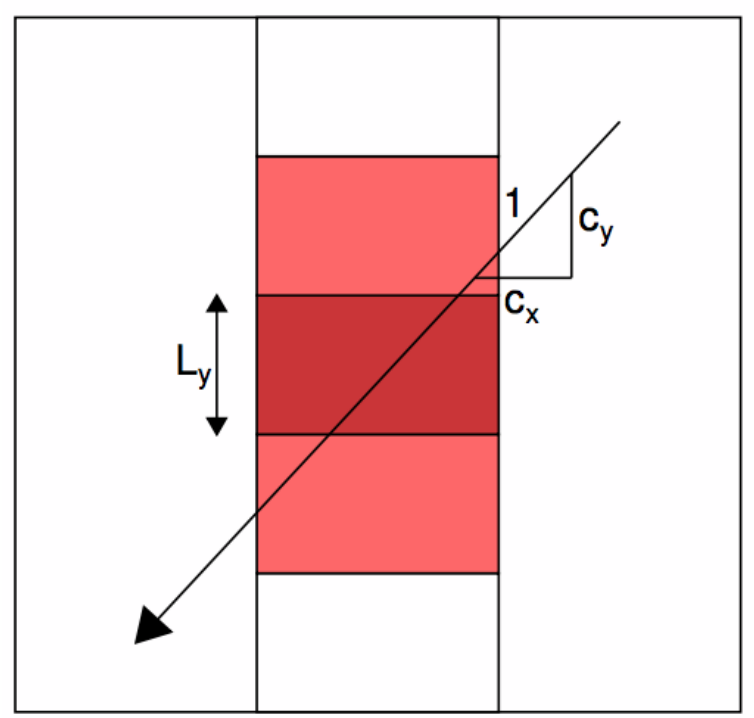

Figure 4.1: An example of a NOvA tricell.

When light travels along the fiber, it gets attenuated. The attenuation calibration corrects the ADC response to get an estimate of the energy deposited in the cell. This is a channel to channel correction performed using cosmic muons. The cell hits from the reconstructed tracks of cosmic ray muons are selected. Then, the uncorrected photoelectrons per path length in a cell is calculated as a function of $W$, the distance of a hit from the center of a cell, as a function of $\mathrm{dE} / \mathrm{dx}$. Based on this a correction is applied as a function of W.

The relationship between the $\mathrm{dE} / \mathrm{dx}$ and $\mathrm{W}$ is characterized by the exponential functions of the form shown in Eq.4.1.

$$
y=C+A\left(\exp \left(\frac{W}{X}\right)+\exp \left(\frac{L+W}{X}\right)\right)
$$

where $y$ is the response, $L$ is the length of the cell, $C, A$ and $X$ are arbitrary constant scale factors.

For the hits which happen deep inside a cell, photons mostly enter the fiber, whereas the hits at the end of the cells are lost. This effect of hits lost at end of the cells is 
termed as "roll-offs" and is modeled by a fourth degree polynomial and this adds to the exponential correction. The empirical form of roll-off is given in Eq. 4.2,

$$
y=\left\{\begin{array}{cc}
1-\alpha_{R}\left(W-W_{R}\right)^{4} & W>+W_{R} \\
1 & \text { otherwise } \\
1-\alpha_{L}\left(W-W_{L}\right)^{4} & W<-W_{L}
\end{array}\right.
$$

Where $W_{L}$ and $W_{R}$ are the onset positions. The fiber inside a cell usually lies in the corners of a cell, but sometimes it may be at the center of a cell which results in the collection of more light. There are relatively large residuals in those channels. The Locally Weighted Scatterplot Smoothing fit used to correct for such irregularities.

The full fit for a channel has seven free parameters which are saved in the calibration database which can be used during the reconstruction. Figure 4.2 shows the full fit for one channel in both ND and FD. After the attenuation calibration, energy per cell stored as a corrected PE, PECorr.
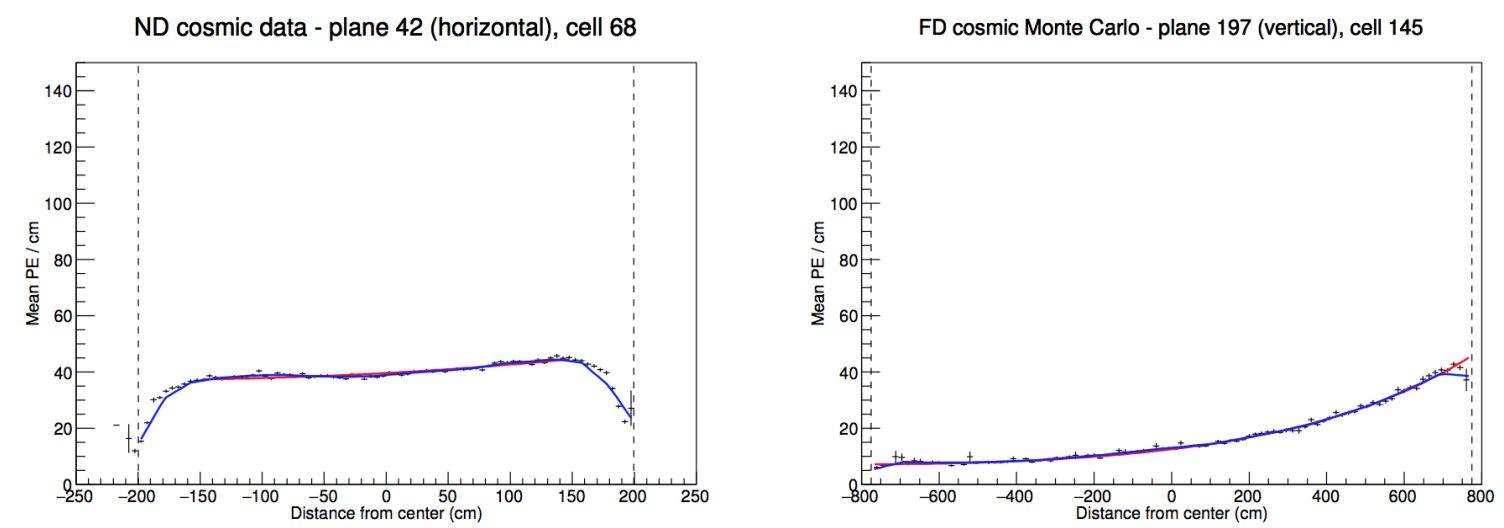

Figure 4.2: The full attenuation fit for ND (left) and FD (right) including the LOWESS fit from data. The blue curve is the full attenuation fit and red curve is the double exponential fit.

\subsubsection{Absolute Calibration}

The absolute calibration provides a scaling factor, independent of the channel, which is used to convert the attenuation corrected energy deposition in units of PECorr $/ \mathrm{cm}$, corrected photoelectrons per length, to physically meaningful units, GeV. This is the last step in the process of calibration. Cosmic muons are used as the standard candle to do this calibration. Specifically, stopping muons are used, that start outside the detector 
and stop inside the detector since the energy loss of muons is well understood following the Bethe-Block curve. The hits which passed the selection criteria are used for the absolute energy calibration. The mean of the distribution of detector response in Muon Energy Units, which are energy deposits of a lowest ionizing particles, for MIP hits in both MC and data is measured. Then, the mean of the distribution of true energy deposits from the $1 \mathrm{~m}$ tracks window from the end point of stopping muons, as shown in Fig. 4.3 provides a conversion factor between the detector response and true energy deposited in the scintillator. The results after the absolute calibration are shown in Fig. 4.4. The correction factor is defined as

$$
\text { Calorimetric energy scale }=\frac{M E U_{\text {truth }}}{M E U_{\text {reco }}}
$$

where $M E U_{\text {truth }}$ is the mean of the distribution of $\mathrm{MeV} / \mathrm{cm}$ in $\mathrm{MC}$ and $M E U_{\text {reco }}$ is the mean of the distribution of PECorr/cm in both data and MC.

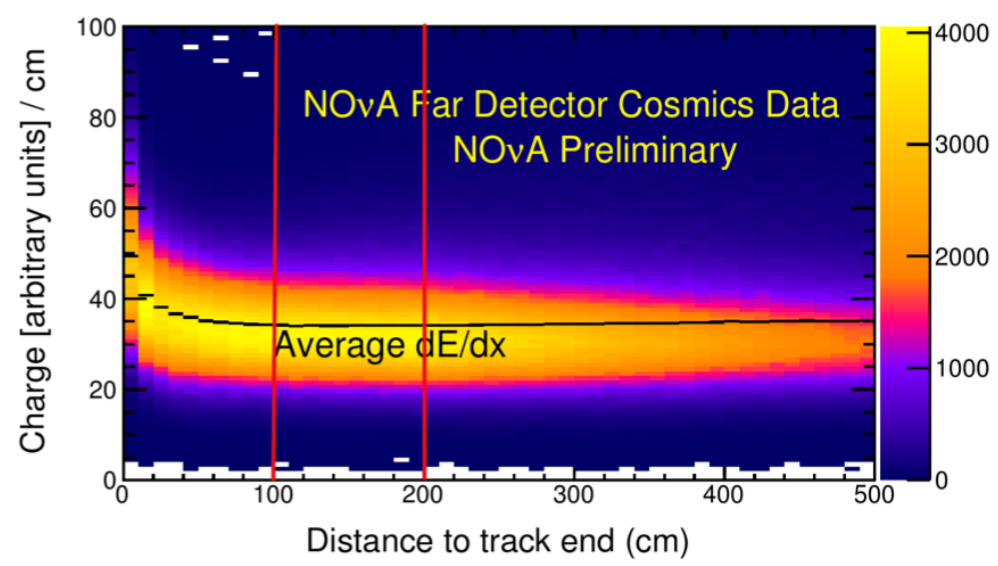

Figure 4.3: The $\mathrm{dE} / \mathrm{dx}$ of a stopping muon measured in FD cosmic data as a function of distance from the end of the track.

\subsection{Event Reconstruction}

Reconstructing a neutrino interaction from cells with energy deposits into tracks and showers which correspond to specific particles, is a challenging task. It's a multi-step process as I will describe in the following sections. 

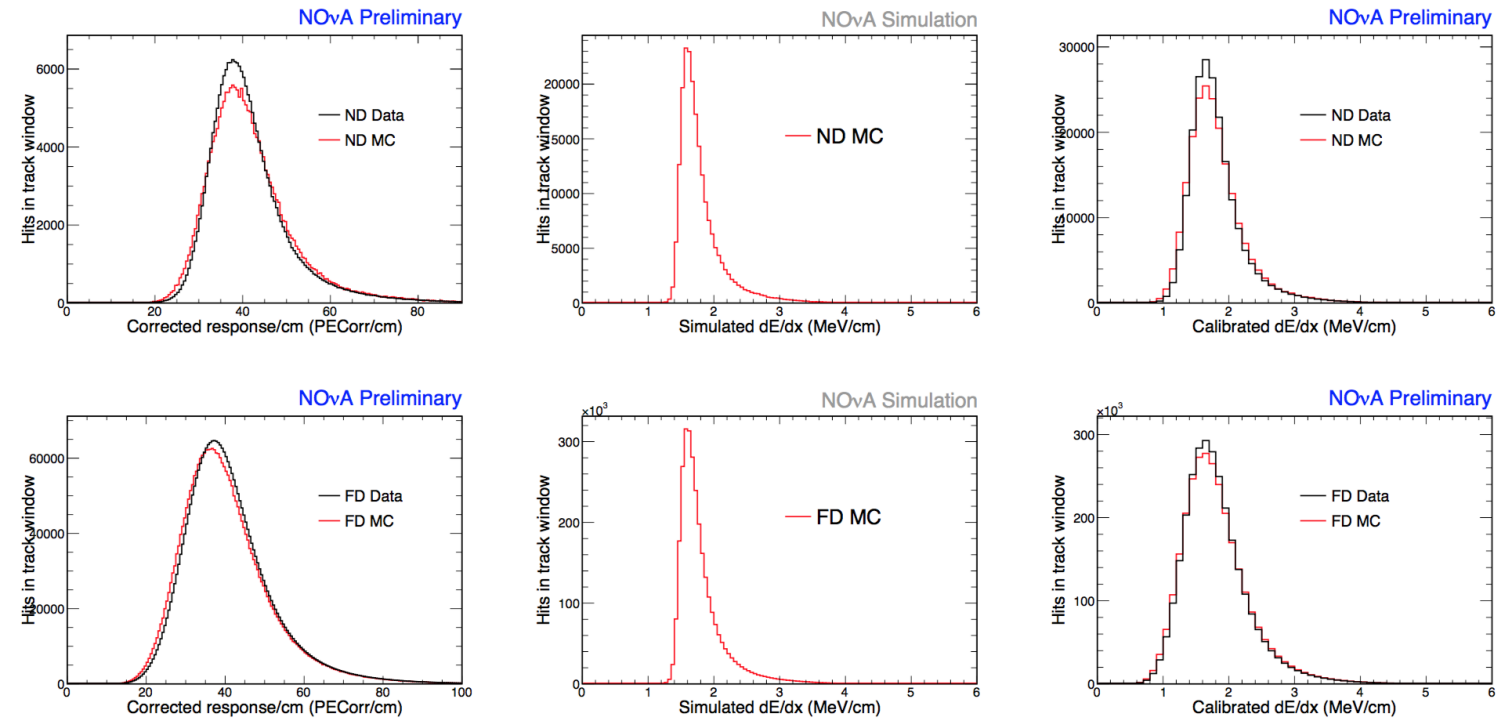

FIgURE 4.4: This plot shows the attenutaion corrected response in $\mathrm{dE} / \mathrm{dx}$ for both near detector (top) and far detector (bottom). The difference between the data and

$\mathrm{MC}$ is used to find a scale factor for the conversion of PECorr $/ \mathrm{cm}$ to $\mathrm{MeV} / \mathrm{cm}$.

\subsubsection{Clustering Algorithm}

Grouping the hits at the lowest level in NOvA is called the "slicing" which is implemented using the Slicer $4 D$ clustering algorithm [102]. The slicing algorithm is based on the Density-Based Spatial Clustering of Application with Noise algorithm which is explained here [103]. This algorithm begins clustering by looping over all the points in the parameter space. The points are categorized into core points and border points. The core points are those having more than a certain minimum number of neighbors within a certain distance, $(\epsilon)$, from itself. The border points are those who have a smaller set than a certain minimum number of neighbors. If the point is in neither of these categories is defined as noise. The algorithm expands the cluster after finding the core point if it finds a neighbor within $\epsilon$, it adds that point and neighbor to the cluster and repeats this for every neighbor until it hits the border point. After it hits border point, it returns to the original list of points and starts clustering again.

The distance between two points, $\epsilon$, is what determines whether the two points are neighbors or not. This is a free parameter, it can be defined as shown in Eq. 4.4,

$$
\epsilon=\left(\frac{\Delta T-\Delta \vec{r} / c}{T_{\text {res }}}\right)^{2}+\left(\frac{\Delta Z}{D_{\text {pen }}}\right)^{2}+\left(\frac{\Delta X Y}{D_{\text {pen }}}\right)^{2}+\left(\frac{P E_{\text {pen }}}{P E}\right)^{2}
$$

Where $T_{\text {res }}$ is the timing resolution of two hits added in quadrature, $D_{\text {pen }}$ and $P E_{\text {pen }}$ are the distance penalty and $P E$ penalty which is free parameters, $P E$ is the number of 
photo-electrons for both hits added in quadrature. For the hits, in the same view $\Delta \vec{r}$, the difference in the position of two hits, is going to be 2-dimensional, and for opposite views it 1-dimensional. The PE spectrum falls off as $P E^{-2.5}$ for noise hits, and this term is used to suppress the noise contribution.

The slicing algorithms can be classified using the three numbers, completeness, purity and efficiency. Completeness tells how much of interaction is contained into the slice, purity tells that how much of a single interaction is contained in the slice, and efficiency is how many of the slices have high completeness and high purity out of all the slices. A good slicing module is the one which has the highest score across a wide range of energies, and a good slice is one with high purity and completeness. The tuning of the free parameters in our slicing module is done separately for both ND and FD due to different noise level and timing resolution. The performance of the slicer4D module is shown in Fig. 4.5.
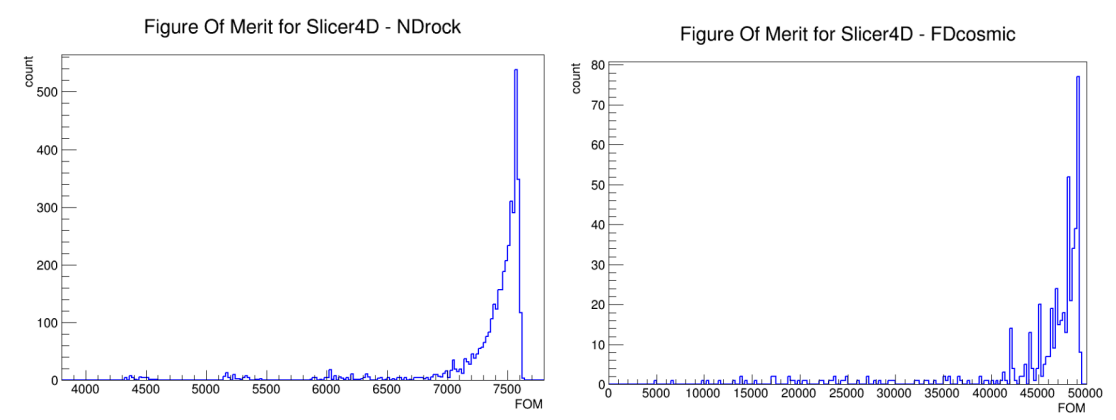

FIGURE 4.5: The performance of Slicer4D module is quantified in terms of function of Figure of Merit (FOM), is the ratio of signal to square root of total selected events, is shown for ND (left) and FD (right).

\subsubsection{Line Finding Algorithm - Multi-Hough Transform}

In the NOvA detectors, the event record is a collection of cell hits recorded in a window of $500 \mu \mathrm{sec}$ around the neutrino spill. Once we cluster the spatially and temporally correlated hits using the clustering algorithm, the next step is to identify the global features within the slice. NOvA uses a modified two-point Hough transform algorithm [104] to find the lines between two hits in both views of the detector XZ and YZ, where $\mathrm{XY}$ plane is transverse to the beam direction, and $\mathrm{Z}$-axis is along the beam direction. Each line is parametrized in polar co-ordinates $\rho$ and $\theta$ as $\rho=\mathrm{x} \cos \theta+\mathrm{y} \sin \theta$. The Hough map is formed by filling up $(\rho, \theta)$ space for every line which passes through two hits in both views with a Gaussian smeared vote as 


$$
\begin{aligned}
\text { vote } & =e^{\frac{\left(\rho-\rho_{0}\right)^{2}}{2 \sigma_{\rho}^{2}}} e^{\frac{\left(\theta-\theta_{0}\right)^{2}}{2 \sigma_{\theta}^{2}}} \\
\sigma_{\rho} & =\frac{3}{\sqrt{12}} \\
\sigma_{\theta} & =\frac{3}{d \sqrt{6}}
\end{aligned}
$$

where $\rho_{0} a n d \theta_{0}$ are the mean of $\rho a n d \theta$, and $d$ is the distance between two hits. Let's assume every particle has one line of path. The lines between hits on the actual line of path results in peaks in the Hough space with a weighted mean of $\rho$ and $\theta$. If the peaks are above a threshold value, then the corresponding lines are considered to be reconstructed as a line. The threshold is set to an average bin height in Hough space for a slice.

There can be multiple Hough lines above the threshold value. We apply a procedure to keep a certain number of Hough lines and remove the remaining lines. We first identify the best Hough line with the tallest peak by iterating over the Hough lines and remove the hits associated with this line. A new Hough map is formed with the remaining hits, and we find the next best Hough line which also arises from the physics events. The iteration ends when there are no significant peaks left in the $\left(\rho_{0}, \theta_{0}\right)$ space as shown in Fig. 4.6. A maximum of 10 lines can be kept in every slice. This procedure ensures an efficient reconstruction of a neutrino event. These Hough lines are given to a vertex finding algorithm as input. The performance of the Hough transform is measured by the perpendicular distance between the Hough lines to the MC true vertex.
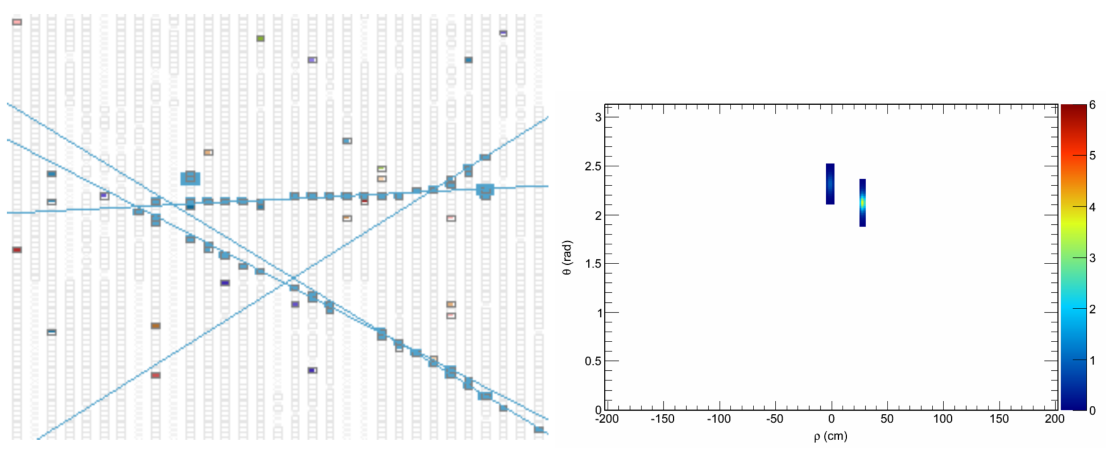

Figure 4.6: The Hough lines after the four iterations (left) and the Hough space $(\rho, \theta)$ on right.

\subsubsection{Vertex Identification}

The next step in the NOvA event reconstruction is to find the neutrino interaction vertex. The Elastic Arms algorithm [105], also known as deformed templates, is used 
to find the interaction vertex without prior knowledge of its location. As we mentioned in the previous section, Hough lines are the input to this algorithm. It is assumed that vertex is at the point of neutrino interaction and a collection of "arms," a collection hits with some direction, extend from the vertex. The Energy Cost Function $E$ defined in Eq. 4.8 is used to locate the true vertex and find arms directions. $N$ and $M$ are the number of hits and number of arms respectively.

$$
E=\sum_{i=1}^{N} \sum_{a=1}^{M} V_{i a} M_{i a}+\lambda \sum_{i=1}^{N}\left(\sum_{a=1}^{M} V_{i a}-1\right)^{2}+\frac{2}{\lambda_{v}} \sum_{a=1}^{M} D_{a}
$$

The first term describes the association of hits with arms. This term is minimized when the arms pass through the hits. The second term is a penalty term for the hits not associated with arms. The third term adds a penalty for arms whose first hits are far from the event vertex with the of lack prior knowledge of vertex location. $M_{i a}$ is the distance between the $i^{t h}$ and $a^{t h}$ arm, $V_{i a}$ is the strength of association between the $i^{\text {th }}$ and $a^{\text {th }}$ arm which satisfies $0<\sum_{a=1}^{M} V_{i a} \leq 1$, is given by

$$
V_{i a}=\frac{e^{-\beta M_{i a}}}{e^{-\beta \lambda}+\sum_{b=1}^{M} e^{-\beta M_{i b}}}
$$

where $\lambda$ and $\lambda_{v}$ control the strength of penalty terms. $\beta$ represents the range of influence of arms which depends on temperature as $1 / T$, a long range at high temperature and short range at low temperatures. The factor $e^{-\beta \lambda}$ represents the likelihood that the hit considered to be noise. The fit procedure uses ROOT's MINUIT class starts with high temperatures to avoid the local minima and continues until it finds the minimum value of energy function which happens at low temperatures. The initial seed for the vertex and arm directions is chosen for which the energy function $E$ is minimum to reduce the computational burden while doing the fitting. The resolution of the vertex position achieved with an algorithm is about 10 to $18 \mathrm{~cm}$ for a variety of neutrino interaction types.

\subsubsection{Cluster-Vertex Matching}

Next in the reconstruction is to find the hits associated with a vertex. FuzzyKVertex [106] is the algorithm which clusters the hits into prongs associated with a vertex. The input for this algorithm is a vertex from the ElasticArms algorithm and a list of cell hits. ElasticArms does well in finding the vertex but is unable to deduce the clear associations between the hits and a vertex. FuzzyKVertex assumes the vertex is the only vertex for the event and all cell hits are associated with this vertex. 
This algorithm is seeded with angles corresponding to clusters of high cell activity using the density matrix containing 360 bins between $-\pi$ and $\pi$,

$$
w_{k}=\sum_{i}^{n} e^{-\left(\frac{\theta_{i}-\theta_{k}}{\sigma}\right)^{2}}
$$

and

$$
\theta_{k}=-\pi+\frac{k \pi}{180}
$$

and $0 \leq k<360$. The cluster membership of cell hits is assigned by calculating first the angular separation between the cell hits and cluster centers as given in Eq. 4.11, at location $x_{j}$ with the clusters whose centers located at $a_{i}$, where $x_{j}-a_{i}$ ranges between $-\pi$ and $\pi$,

$$
d_{i j}=\left(\frac{x_{j}-a_{i}}{\sigma_{j}}\right)^{2}
$$

and calculating the cell membership as

$$
\mu_{i j}=e^{-\frac{m d_{i j} \sqrt{a}}{\beta}}
$$

Where $m$ is the degree of fuzziness of clusters in the slice which at NOvA set at 2, $a$ is the number of clusters, and $\beta$ is the spread of hits around the cluster centers which was set to 4 . Every iteration the cluster centers are updated with

$$
\theta_{i}^{\prime}=\theta_{i}+\frac{\sum_{j}^{n} \frac{\mu_{i j}}{\sigma_{j}^{2}}\left(\theta_{j}-\theta_{i}\right)}{\sum_{j}^{n} \frac{\mu_{i j}}{\sigma_{j}^{2}}}
$$

The process of iteration happens until the deviation between the old, and new centers are $<10^{-7}$ radius. After prong as determined by this algorithm, they are then matched with prongs in another view to make the $3 \mathrm{D}$ prongs which are used in the analysis.

\subsection{Particle Identification - Convolutional Visual Networks}

After we found prong, we still have a challenging task which is the particle identification classifying particles into different types. This can be done by using the algorithms like the Boosted Decision Trees [107], K-Nearest Neighbors [108] and Multilayer Perceptrons $[109,110]$. These classifiers are trained on features like the reconstructed objects such 
as tracks, prongs, showers. These machine learning classifiers are highly sensitive to the reconstructed event features and the limited number of input event features itself makes their applications limited. Recently, the field of computer vision has made the advancement of using feature extraction using machine learning algorithms called Convolutional Neural Networks (CNN).

Deep learning [111] is the next level machine learning algorithm which has many advances that it can mitigate the drawbacks of traditional methods. Deep learning uses the multilayered networks which are very successful in pattern recognition and image processing. The Convolutional Neural Networks (CNN) are superior to the neural networks with fully connected layers because CNN's consider the spatial structure of input images. These networks adopted a particular architecture which makes it faster to train.

The CNN consists of several convolutional layers that extract features from the input image. The input data passed to CNN has three dimensions which are the width, height and a channel number which is like RGB channels of a color image. The input data is transformed to a convolutional layer according to as

$$
(k * g)_{p, q}=\sum_{l=-\infty}^{\infty} \sum_{m=-\infty}^{\infty} \sum_{n=0}^{c} k_{l, m, n} g_{p-l, q-m, n}
$$

Where $k$ is referred as kernel or filter consists of filter weights, $g$ is a sequence of image pixel intensities, $(p, q)$ is the position of the hidden neuron in the hidden layer. The filter weights in the kernel $k$ trained to identify the features in the image. Every hidden neuron represents the maximum value in the local receptive filed of the input image. The local receptive field is a $n \times m$ sub-region in the input image. Every hidden layer extracts one feature of the image says, edge in the image or some other feature. This can be called a feature map between the input layer and the hidden layer. There can be as many as such feature maps extracting various features of an input image. Every feature map is stored in the channel dimension of the hidden layer output. With the increase of every hidden layer with many feature maps, the memory needed for the network operations can be exceptionally high. So, CNN's apply a technique called pooling that reduces the dimension of the input layer by employing a max pooling technique. In max pooling, every $n \times m$ sub-region in the previous convolutional layer is replaced with a hidden neuron corresponding to a maximum weight. The pooling layer is formed after every convolutional layer. To avoid the information loss, the pooled regions can be chosen to overlap.

So, CNN's have an input image, many convolutional layers, and pooling layers. The final layer is fully connected to every neuron in previous max pooled layer. In NOvA, we use 
a deep network architecture developed by Google team with 22 hidden layers which are GoogLeNet [112]. GoogLeNet uses a mechanism called network-in-network (NIN) in which the learning capacity in each map is augmented by reducing the dimensionality in parallel. The NIN consists of many sub-networks resembling the conventional CNN convolutional networks at various scales to extract the complex features of images. Each sub-network is called an inception module. The inception modules extract features by applying filters at various scales. GoogLeNet also uses local response normalization in which the response from every cell is normalized relative to the adjacent feature maps.

For NOvA, we use a specific CNN algorithm, called Convolutional Visual Networks $(\mathrm{CVN})$ for the event classification. The CVN is trained and tested on the simulated events in NOvA. The NOvA CVN is implemented using the caffe [113] framework which provides fast training on graphics processing units. The NOvA CVN architecture as shown in Fig. 4.7, is different from the GoogLeNet architecture in certain ways. First, NOvA event interaction has two separate views, $\mathrm{X}$-view and $\mathrm{Y}$-view. Each view is trained separately using the three inception modules. We concatenate the two views to obtain the final output which is then fed to the final inception module to extract the combined features. The average pooling is done on the final output for further downsampling. The final score is obtained from the softmax function which classifies the event.

We classify events both into flavour or neutral current and by the neutrino interaction mode, Quasi-elastic, resonant, Deep inelastic scattering as based on definitions from GENIE. We train the CVN using the Mini-Batch method [114] simultaneously over 32 training samples. Multiple regularization techniques are used to ensure that the trained CVN could work for other samples. The over training can be reduced by making the training sample large enough. To augment the training sample, we include a Gaussian noise with $1 \%$ variation in the pixel intensities. We apply CVN to a $\nu_{\mu} \mathrm{CC}$ interaction as shown in Fig. 4.8. The top right plot is a $7 \times 7$ convolution layer with 64 feature maps extracting various features of a trained network and the bottom right shows the final output after applying the 64 filters to the $\nu_{\mu} \mathrm{CC}$ interaction.

\subsection{Energy Reconstruction}

The NOvA experiment consists of tracking calorimetric detectors. The calibration of NOvA detectors is done using the cosmic ray muons. The event topology and energy loss $(d E / d x)$ is different for $\nu_{e}$ and $\nu_{\mu}$ interactions which means they have different energy reconstruction procedures. Each reconstructed object contains the energy deposition in the cells by both electromagnetic and hadronic components. The energy reconstruction 


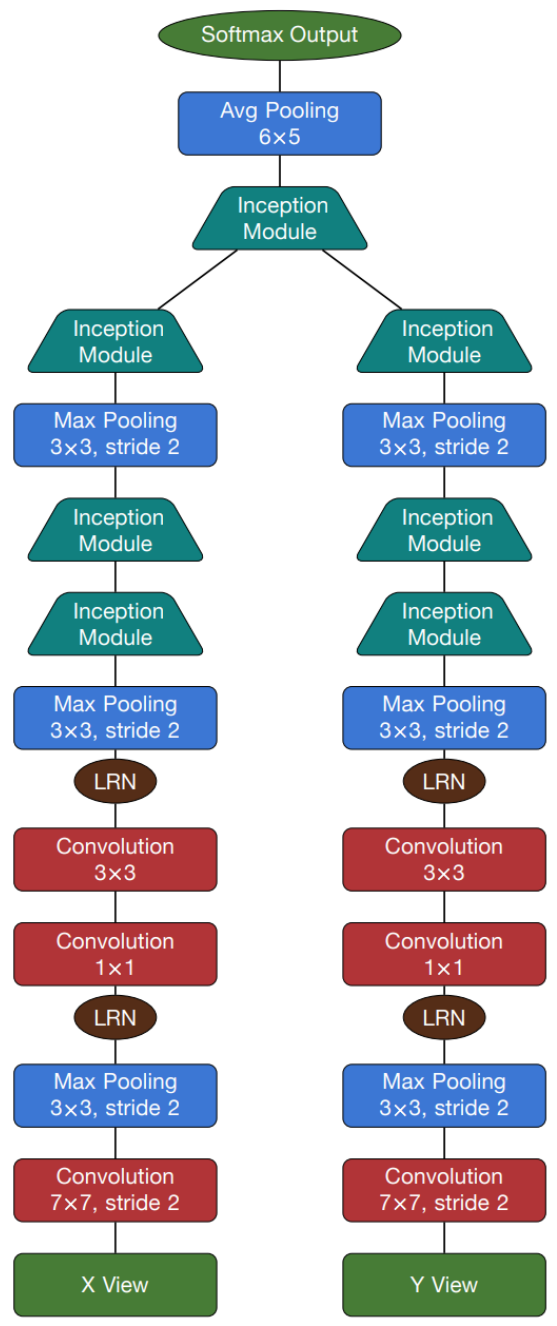

Figure 4.7: Shows the NOvA CVN architecture.

separate outs the EM from the hadronic component and uses this information to evaluate the total energy deposited in one neutrino interaction.

\subsection{1 $\nu_{e}$ Energy Estimator}

The electron neutrino energy reconstruction uses Convolutional Visual Network [115] prong identification. The $\nu_{e}$ energy reconstruction [116] starts by looping over all the prongs in a slice where every prong is assigned a value to whether the prong is made by electromagnetic or by hadrons. A given prong is electromagnetic if the sum of CVN electromagnetic is greater than the sum of hadronic prongs.

The electromagnetic component of energy, $\mathrm{E}_{\mathrm{EM}}$, is the sum of all electromagnetic prongs and the hadronic component, $\mathrm{E}_{\mathrm{Had}}$, is the remaining energy in a slice. The relation between the EM and hadronic components weight by the average true neutrino energy 

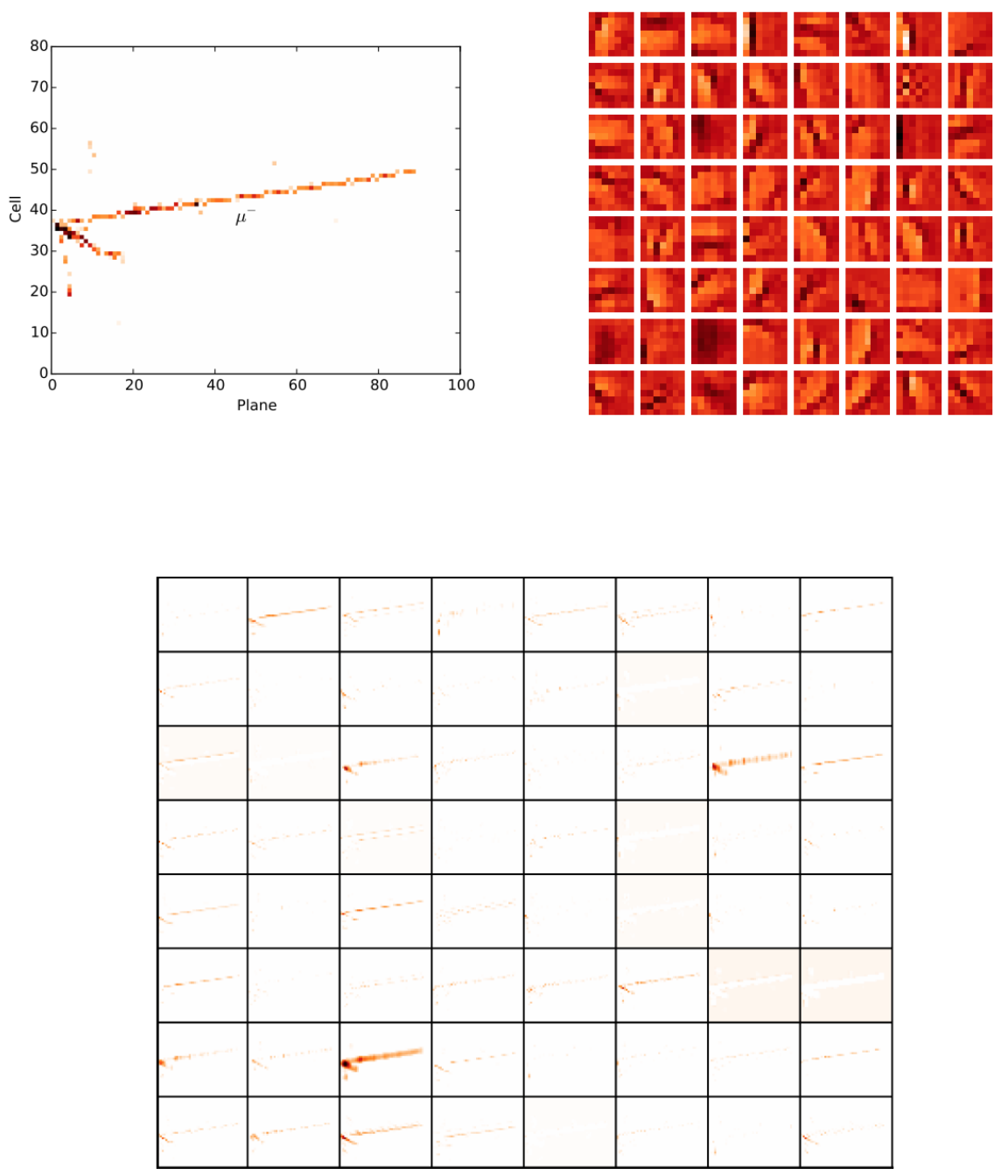

Figure 4.8: The example NOvA CVN applied to a $\nu_{\mu} \mathrm{CC}$ interaction.

is shown in Fig. 4.9. To make the fit independent of true neutrino energy, we apply a flat weight for every event in true energy. This flat weight is obtained by normalizing the true energy distribution. This spectrum is later used to fit the weighted average true energy with a function of the form $\mathrm{E}_{\text {reco }}=a x+b y+c x^{2}+d y^{2}$ where $a, b, c$ and $d$ are fitting parameters determined after the reconstructed energy fit and $x, y$ are $\mathrm{E}_{\mathrm{EM}}$ and $\mathrm{E}_{\mathrm{Had}}$ respectively. After the fit, we obtain the fitting function which is the total reconstructed energy, $E_{\text {reco }}$ as

$$
\mathrm{E}_{\text {reco }}=\frac{1}{1+0.057}\left(0.996 \mathrm{E}_{\mathrm{EM}}+0.869 \mathrm{E}_{\mathrm{Had}}+0.025 \mathrm{E}_{\mathrm{EM}}^{2}+0.504 \mathrm{E}_{\mathrm{Had}}^{2}\right)
$$

The energy resolution is plotted for the $\nu_{e}$ events is shown in fig. 4.10. We can also see the energy resolution as a function of true neutrino energy shown in fig. 4.11. This energy estimator is mostly flat resolution across the NOvA interested energy region. 


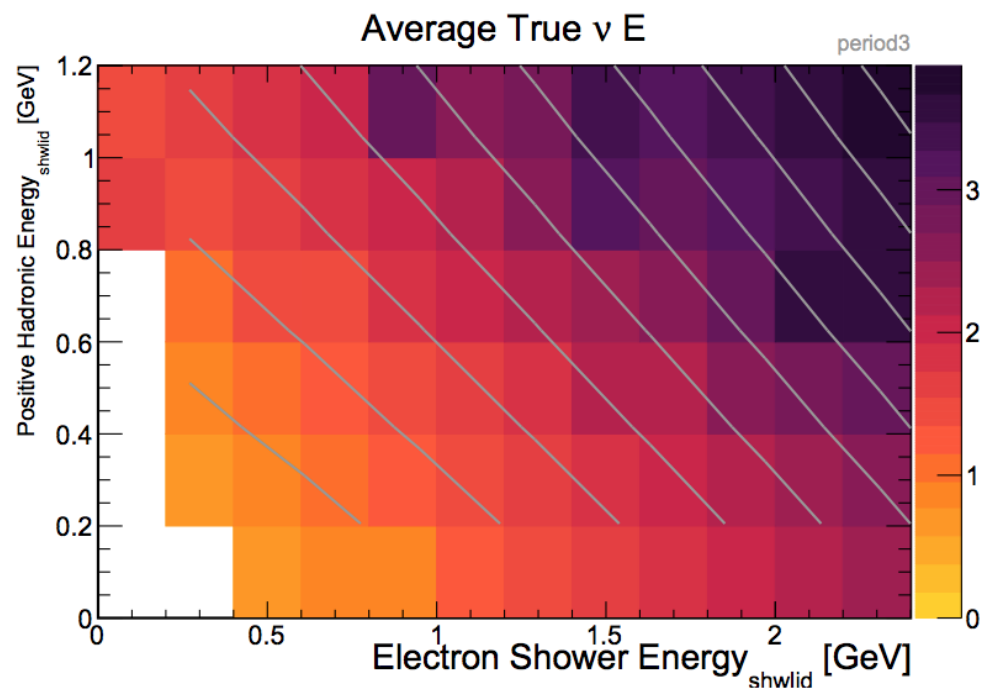

FiguRE 4.9: The relationship between electromagnetic component energy and the hadronic component of energy for the Near Detector.

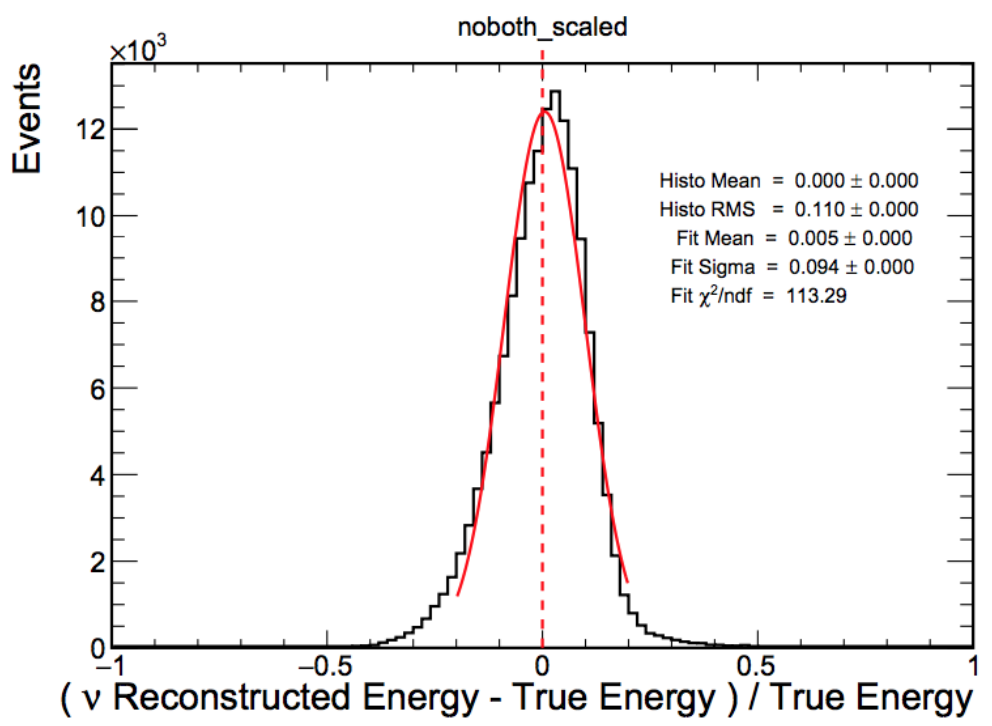

Figure 4.10: The energy resolution using $\nu_{e}$ energy estimator for the Near Detector.

\subsection{2 $\nu_{\mu}$ Energy Estimator}

The $\nu_{\mu}$ energy estimator [117] is used to reconstruct the energy of the $\nu_{\mu}$ induced interaction in the detector. This was implemented in the NOvA software package called NumuEnergy. The same procedure is used at both detectors except for the treatment of event in the ND muon catcher. The rate of energy deposition of muons in the muon catcher is radically different from that of in the active region. The $\nu_{\mu}$ energy reconstruction reconstructs the energy of muon and hadronic system which is the sum of all hits 


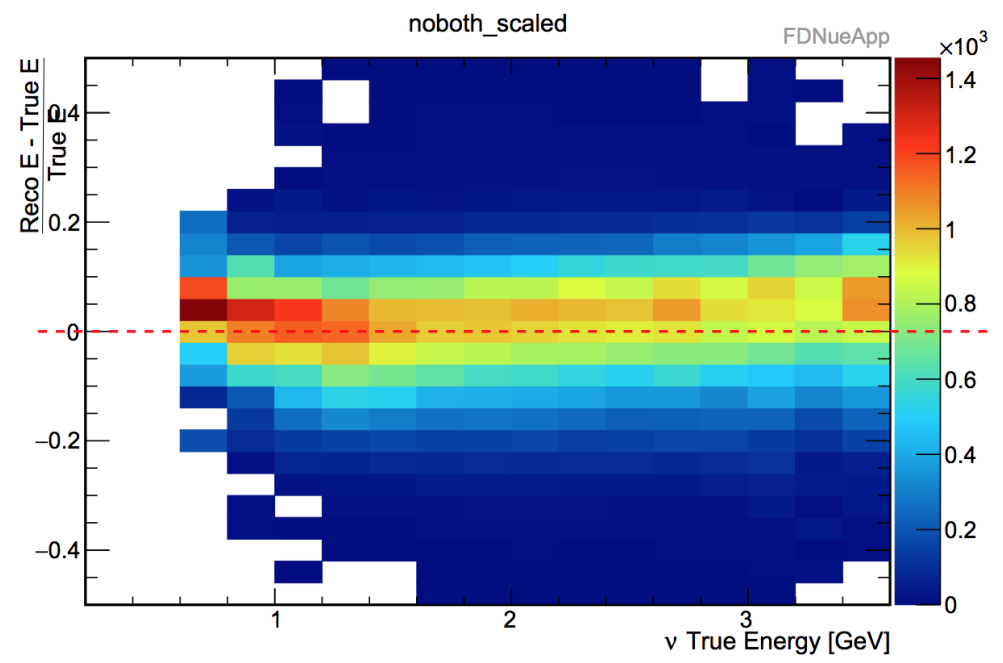

FIgURE 4.11: The energy resolution as a function of true enutrino energy using $\nu_{e}$ energy estimator for the Near Detector. We clearly see that energy resolution is independent of true energy.

not from the muon separately and adds them together for the total $\nu_{\mu}$ deposited energy.

\subsubsection{Muon Energy}

Muons are minimum ionizing particles which have roughly constant energy loss rate across the detector medium. The energy loss for muons per unit path length is same for a wide range of momenta. The relationship between the true and reconstructed energy is modeled by fitting a Gaussian to the true muon energy distribution for each bin of reconstructed energy. As the muon starts losing its energy, the linear relationship between reconstructed track length and true muon energy is no longer valid at lower momenta. Therefore a piecewise linear fit is used as shown in Fig. 4.12 and 4.13. This fit gives the relationship between the reconstructed track length and reconstructed muon energy.

The ND consists of a fully active region and muon catcher for the neutrino interactions which start in the active region and ends in the muon catcher, a slightly different procedure is used to get the reconstructed muon energy because the energy deposition rate is different in the muon catcher. In such cases, a track is divided between the active region and muon catcher. First, we make the energy estimator for muons fully contained in the active region. Then, we use this energy estimator to form the energy estimator for tracks in the catcher. Then, the length of the active region and the length of muon catcher are added together to get the total track length. This total length is then converted the reconstructed energy using the far detector. 

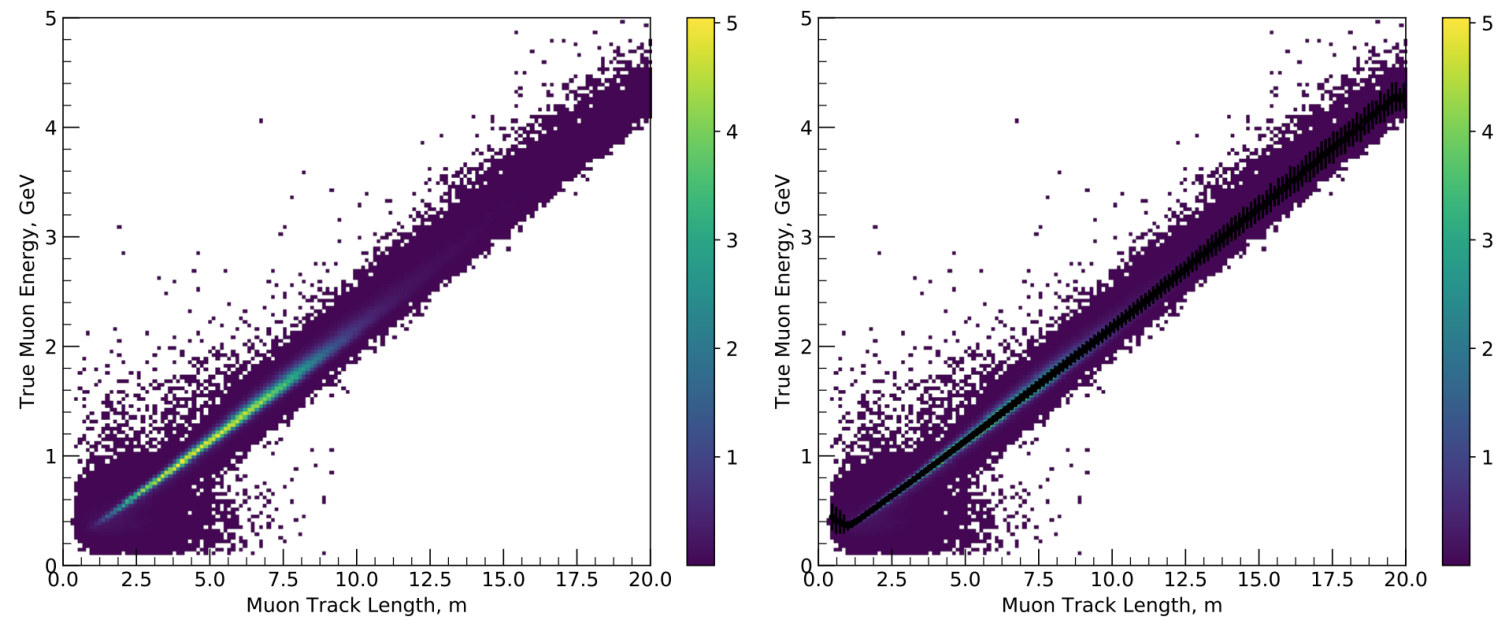

Figure 4.12: The 2D histogram of reconstructed muon length vs true muon energy (left). A gaussian fit is performed for the true energy of muons at every vertical slice.
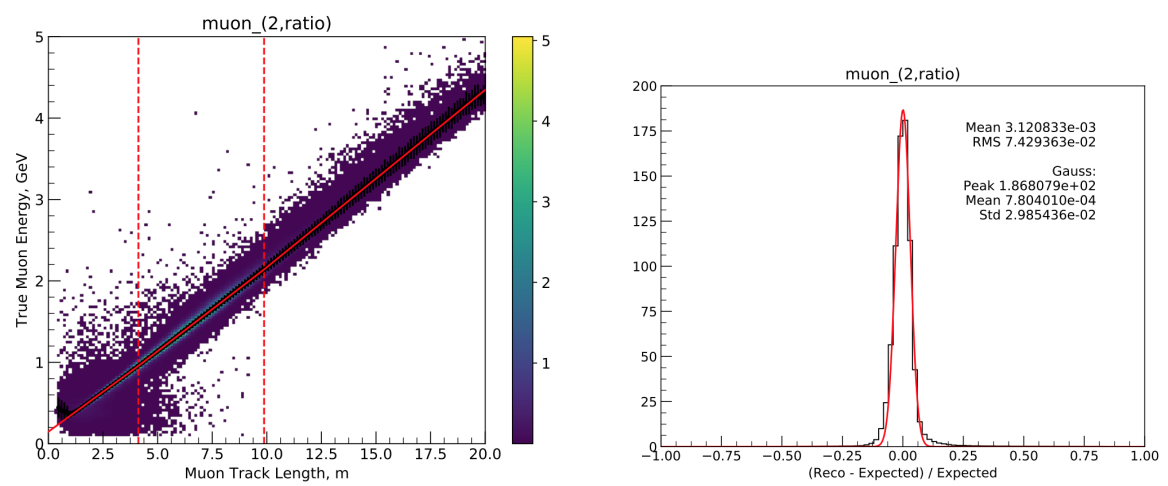

Figure 4.13: A multi linear fit is performed to find the peak of gaussian profile at every vertical slice. The position of dotted lines are where the each spline ends (left).

The energy resolution of muon after the fit (right).

\subsubsection{Hadron Energy}

After the muon energy reconstruction, we move on to the reconstruction of hadron energy. Since only low energy events are contained in the ND and because of limited energy range, we use a two-line fit. The visible hadronic energy is the sum of energy from hits not on the muon track and sum of energy of hits in the vertex region which are not MIP hits. The same fitting procedure is used as is used for muons which are shown in Fig. 4.14 and 4.15. 

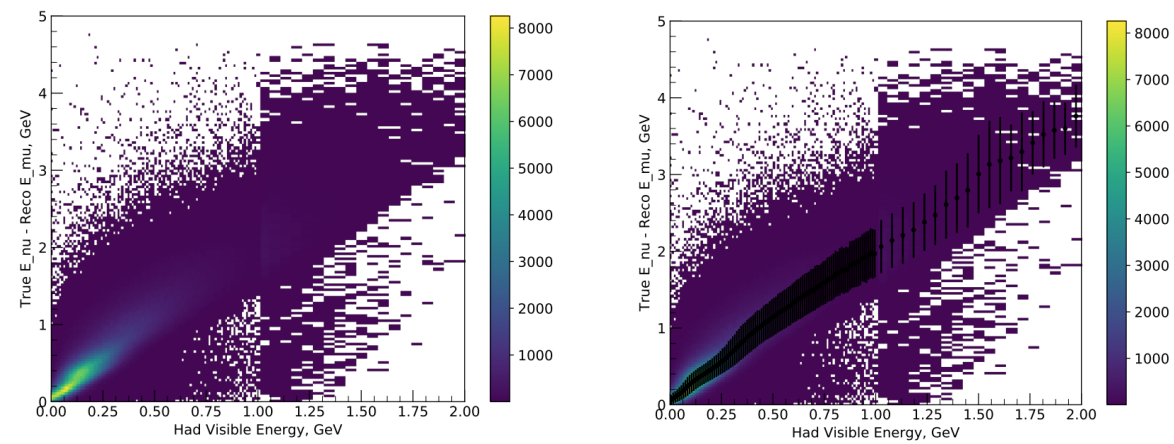

Figure 4.14: The 2D histogram of reconstructed muon length vs true muon energy (left). A gaussian fit is performed for the true energy of muons at every vertical slice (right).
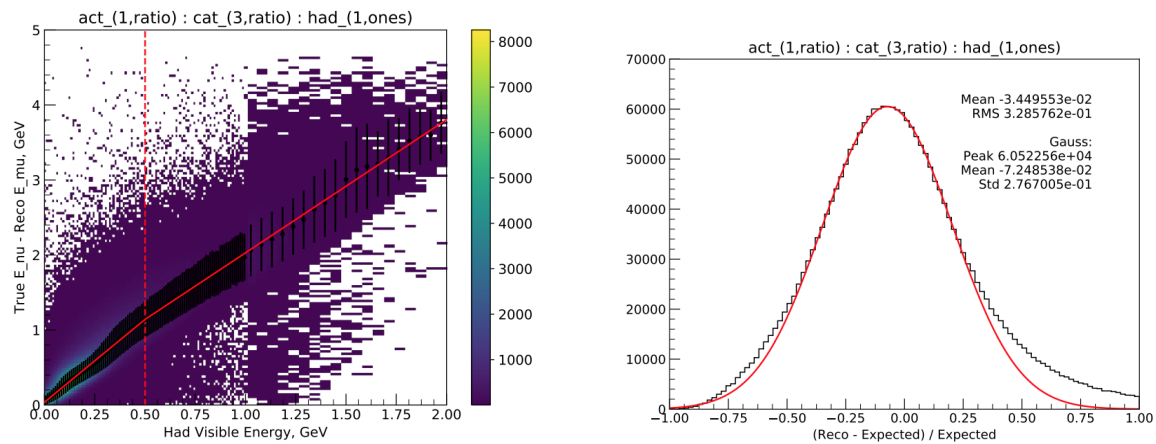

FIGURE 4.15: A multi line fit is performed to find the peak of gaussian profile at every vertical slice. The position of dotted lines are where the each spline ends (left). The energy resolution of hadron after the fit (right).

\subsubsection{Muon Neutrino Energy}

Having the reconstructed muon and hadron energy, we then get the reconstructed neutrino energy by summing these two energies. The relation between the true and reconstructed neutrino energy is shown in Fig. 4.16 and the energy resolution is shown in Fig. 4.17.

\subsubsection{Track Reconstruction}

Track reconstruction is done in NOvA using the two algorithms, KalmanTrack and KalmanTrackMerge. The input to the KalmanTrack algorithm is a cluster of hits from slices made by Slicer. Kalman filtering is the basic ingredient in Kalman tracker. A track is a continuous collection of hits made by a single particle traveling through the detector. The output from this algorithm is a set of $2 \mathrm{D}$ tracks in either view, associated with a vertex in a slice. Then the KalmanTrackMerge combines the matched 2D tracks from different views to make 3D tracks. Every track contains the start of the track, end of the track and all the cell hits on the track. This analysis only uses the 3D tracks. 


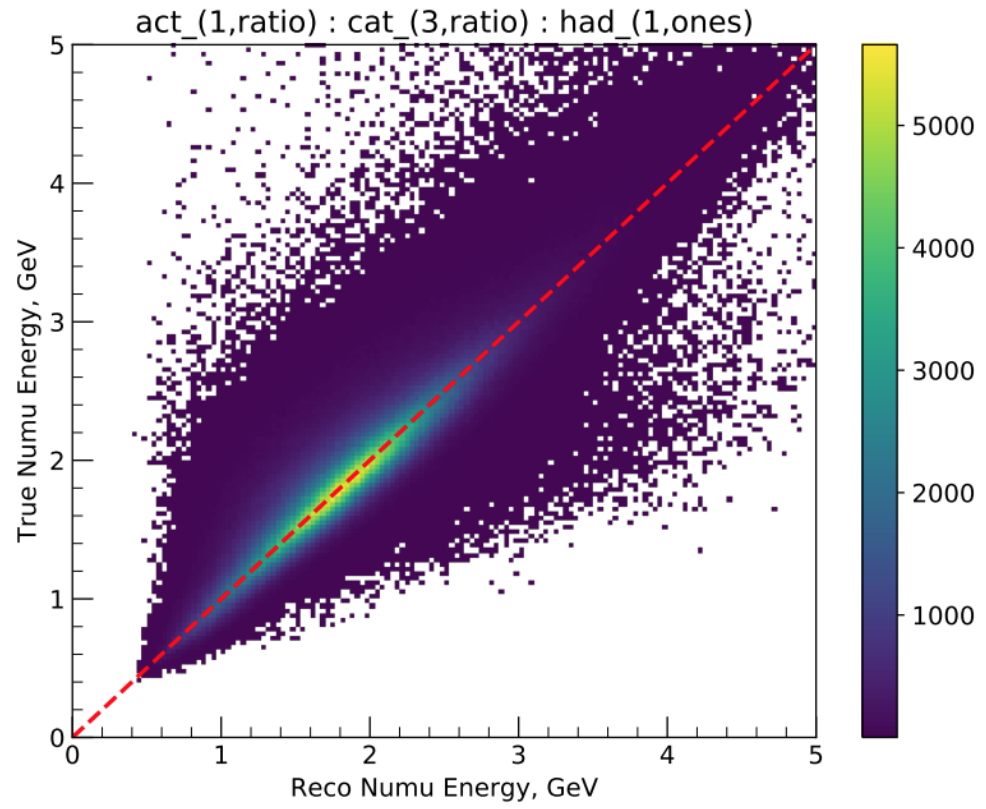

FiguRE 4.16: Neutrino true energy versus reconstructed energy.

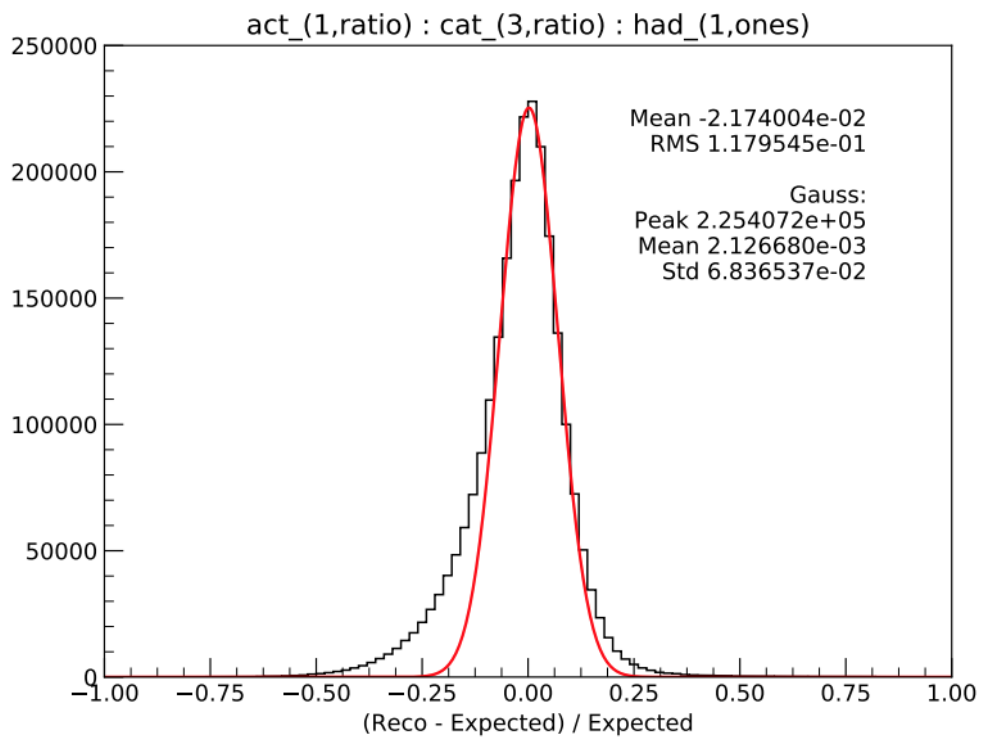

Figure 4.17: The resolution of the neutrino energy.

The Kalman tracker starts with all the hits in either XY or YZ view in a cluster. A pair of hits that are less than three planes, in one view, forms seeds for the tracker. Through this pair of hits, test tracks are propagated to the next plane using the current position of the track, and its slope. If the hits found in the next plane satisfying the conditions on being on track, it is added to the track, and this process continues until it doesn't find any hits in consecutive three planes. The hits are added to the track only if the $\Delta \chi^{2}$ of the track fit after adding this hit is less than 8. After the complete track is found, 
this process is redone starting from the other end of the track without adding new hits to check whether the added hits can now be rejected. Tracks must be at least four hits long. This process is redone using the other seeds that were not on any tracks to find the $2 \mathrm{D}$ tracks. A good track is a long track with maximum reconstruction efficiency.

Once all 2D tracks have been made in each view, KalmanTrackMerge forms a 3D track from $2 \mathrm{D}$ tracks from both views which are consistent with each other. The consistency is determined by a score which is defined as

$$
\text { score }=\frac{\left(Z_{X Z}^{\mathrm{start}}-Z_{Y Z}^{\mathrm{start}}\right)+\left(Z_{X Z}^{\mathrm{end}}-Z_{Y Z}^{\mathrm{end}}\right)}{\text { Length of overlap in } \mathrm{Z}}
$$

In some cases, a long $2 \mathrm{D}$ track in one view is matched to multiple tracks in another view. It might be possible if the scattering of the particle broke the track into pieces. Merging continues until it finds tracks which overlap in $\mathrm{Z}$. 



\section{Chapter 5}

\section{Systematic Uncertainties}

\subsection{Systematics Methodology}

Systematic uncertainties are included in the Near Detector (ND) fit as nuisance parameters using the full shape information unless specifically stated otherwise. The scale of the systematics is quantified as the percentage change in the ND prediction of the number of background or signal events to make quantifiable comparisons.

This analysis is a joint fit between $\nu_{e}$ appearance and $\nu_{\mu}$ disappearance which helps for this Near Detector only analysis, as correlated systematics between $\nu_{e}$ and $\nu_{\mu}$ selected samples can cancel or be significantly reduced. Table 5.1 and 5.2 show the list of systematics we consider for this analysis and also indicates the correlated (or not) systematics between the $\nu_{e}$ and $\nu_{\mu}$ channels, respectively. For this analysis, systematics are treated as $100 \%$ correlated or uncorrelated. For a systematic defined as correlated the shift is applied for both the $\nu_{e}$ and $\nu_{\mu}$ prediction for the correlated parameter, whereas for the uncorrelated case, systematic shifts are applied separately to the $\nu_{e}$ or $\nu_{\mu}$ prediction.

For effects such as beam flux, light level simulation, and calibration, uncertainties are evaluated by generating systematically modified Monte Carlo (MC) samples, as are listed in Appendix A, and comparing to the nominal MC. The cross-section and PPFX uncertainties are evaluated by reweighting events in the nominal MC samples for the ND. The systematics due to $\nu_{\mu}$ to $\nu_{e}$ energy bias, acceptance effects, and mis-modeling of rock events, arise from specific studies described later in this note. 


\begin{tabular}{c|cc|c}
\multirow{2}{*}{ Systematics } & Correlated & Systematics & Correlated \\
\cline { 2 - 4 } & Yes & PPFX and Beam Transport & Yes \\
\hline PPFX and Beam Transport & Yes & Cross-section & Yes \\
\hline Cross-section & Yes & Calibration & Yes \\
\hline Calibration & Yes & Light Level & Yes \\
\hline Light Level & Yes & Cherenkov & Yes \\
\hline Cherenkov & Yes & Rock & No \\
\hline Normalization (POT, Mass) & No \\
\hline Energy Bias & Yes & Muon Energy scale & Yes \\
\hline Acceptance & Energy Bias & No \\
\hline Intensity & Acceptance & Yos \\
\hline TABLE 5.1: Summary of all $\nu_{e}$ system- & Intensity & Yes \\
\hline
\end{tabular}
atics.

TABLE 5.2: Summary of all $\nu_{\mu}$ systematics.

\subsection{Summary of Systematics}

A summary of all the systematics studied, showing the percent change in the integral, is given in Table 5.3 for the appeared $\nu_{e}$ and in Table 5.4 for the $\nu_{\mu}$ sample.

\begin{tabular}{|c|cc|cc|cc|cc|cc|}
\hline \multirow{2}{*}{ Syst.Parameter } & \multicolumn{2}{|c|}{$\nu_{e}$ sig } & \multicolumn{2}{|c|}{ Total bkg } & \multicolumn{2}{c|}{$\nu_{\mu}$} & \multicolumn{2}{c|}{ beam $\nu_{e}$} & \multicolumn{2}{c|}{$\mathrm{NC}$} \\
\cline { 2 - 15 } & $+1 \sigma(\%)$ & $-1 \sigma(\%)$ & $+1 \sigma(\%)$ & $-1 \sigma(\%)$ & $+1 \sigma(\%)$ & $-1 \sigma(\%)$ & $+1 \sigma(\%)$ & $-1 \sigma(\%)$ & $+1 \sigma(\%)$ & $-1 \sigma(\%)$ \\
\hline RPAShapeenh2017 & 5.5 & 4.6 & 4.5 & 3.6 & 3.2 & 2.2 & 5.5 & 4.5 & 0 & 0 \\
\hline MECq0Shape & 9.4 & 7.2 & 6.3 & 5.7 & 0.12 & 0.073 & 8.5 & 7.8 & 0.24 & 0.24 \\
\hline MECEnuShape & 4.1 & 4.1 & 2.7 & 2.7 & 0.092 & 0.092 & 3.6 & 3.6 & 0.0069 & 0.0069 \\
\hline DISvnCC1pi & 0.95 & 0.95 & 3 & 3 & 16 & 16 & 1.2 & 1.2 & 0 & 0 \\
\hline MaCCRES & 6.5 & 7.1 & 6.1 & 6.1 & 7.1 & 6.2 & 7 & 7.2 & 0 & 0 \\
\hline MvCCRES & 3.6 & 3.4 & 3.3 & 3 & 4 & 3.5 & 3.8 & 3.4 & 0 & 0 \\
\hline MaCOHpi & 0.81 & 0.85 & 2.3 & 2.4 & 0.081 & 0.081 & 0.83 & 0.85 & 13 & 13 \\
\hline R0COHpi & 0.84 & 0.81 & 2.4 & 2.3 & 0.081 & 0.081 & 0.84 & 0.82 & 13 & 13 \\
\hline MFP_N & 2.6 & 2.6 & 2 & 2.1 & 1.1 & 1.3 & 2.5 & 2.6 & 0.27 & 0.31 \\
\hline Calibration & 0.1 & 1.4 & 0.1 & 1.4 & 10.6 & 16.8 & 3.6 & 3.6 & 10.8 & 13.5 \\
\hline Calibration Shape & 0.01 & - & 1.2 & - & 0.4 & - & 2.9 & - & 7.3 & - \\
\hline Light Level & 1.4 & 0.4 & 0.4 & 2.1 & 3.2 & 0.9 & 0.3 & 2.9 & 2.3 & 1.7 \\
\hline Cherenkov & 0.2 & - & 1.0 & - & 5.1 & - & 1.2 & - & 4.8 & - \\
\hline Summedsmallgenie & 6.7 & 6.7 & 7.2 & 8.4 & 7.9 & 7.9 & 7.7 & 7.7 & 7.9 & 7.9 \\
\hline beamTransportComb & 4.8 & 4.8 & 3.3 & 4.1 & 5.1 & 5.1 & 2.6 & 3.3 & 5.8 & 5.8 \\
\hline ppfx_pc00 & 0.7 & 0.7 & 1.4 & 1.5 & 0.4 & 0.4 & 1.7 & 1.7 & 0.8 & 0.8 \\
\hline ppfx_pc01 & 7.0 & 7.0 & 7.9 & 6.8 & 7.0 & 7.0 & 7.2 & 7.2 & 7.2 & 7.2 \\
\hline ppfx_pc02 & 3.2 & 3.2 & 2.1 & 2.2 & 2.9 & 2.9 & 2.1 & 2.1 & 2.0 & 2.0 \\
\hline ppfx_pc03 & 1.5 & 1.5 & 0.8 & 0.8 & 1.4 & 1.4 & 0.8 & 0.8 & 1.4 & 1.4 \\
\hline ppfx_pc04 & 3.5 & 3.5 & 3.9 & 3.6 & 3.5 & 3.5 & 3.7 & 3.7 & 3.6 & 3.6 \\
\hline
\end{tabular}

TABLE 5.3: Systematic summary of $\nu_{e}$ sample. The top six are large GENIE parameters included as individual pulls. All other GENIE parameters are included in the summed small genie. All five pc for PPFX are shown.

Figure 5.1 shows the effect of all systematics on the resulting predictions. The $90 \%$ C.L. sensitivity to $\theta_{\mu e}$ is shown in Fig. 5.2, comparing the statistics only curve to that when fit with all systematics. 


\begin{tabular}{|c|cc|cc|cc|cc|cc|}
\hline \multirow{2}{*}{ Syst.Parameter } & \multicolumn{2}{|c|}{$\nu_{e}$ sig } & \multicolumn{2}{c|}{ Total bkg } & \multicolumn{2}{c|}{$\nu_{\mu}$} & \multicolumn{2}{c|}{ beam $u_{e}$} & \multicolumn{2}{c|}{$\mathrm{NC}$} \\
\cline { 2 - 13 } & $+1 \sigma(\%)$ & $-1 \sigma(\%)$ & $+1 \sigma(\%)$ & $-1 \sigma(\%)$ & $+1 \sigma(\%)$ & $-1 \sigma(\%)$ & $+1 \sigma(\%)$ & $-1 \sigma(\%)$ & $+1 \sigma(\%)$ & $-1 \sigma(\%)$ \\
\hline DISvpCC2pi & 4.2 & 4.2 & 2.4 & 2.4 & 1.5 & 1.5 & 3.6 & 3.6 & 0 & 0 \\
\hline DISvpNC2pi & 0 & 0 & 2.7 & 2.7 & 0 & 0 & 0 & 0 & 6.4 & 6.4 \\
\hline DISvpNC3pi & 0 & 0 & 2.2 & 2.2 & 0 & 0 & 0 & 0 & 5.3 & 5.3 \\
\hline DISvnCC1pi & 5.6 & 5.6 & 3.2 & 3.2 & 2 & 2 & 4.7 & 4.7 & 0 & 0 \\
\hline DISvnCC2pi & 4.4 & 4.4 & 2.5 & 2.5 & 1.3 & 1.3 & 4.1 & 4.1 & 0 & 0 \\
\hline DISvnNC1pi & 0 & 0 & 2.7 & 2.7 & 0 & 0 & 0 & 0 & 6.4 & 6.4 \\
\hline DISvnNC2pi & 0 & 0 & 2 & 2 & 0 & 0 & 0 & 0 & 4.8 & 4.8 \\
\hline MaCCRES & 15 & 12 & 8.3 & 7.1 & 8 & 8.6 & 10 & 9 & 0 & 0 \\
\hline MvCCRES & 8.3 & 7 & 4.8 & 4 & 4.5 & 4.2 & 5.9 & 5.1 & 0 & 0 \\
\hline MaNCRES & 0 & 0 & 5.7 & 4.5 & 0 & 0 & 0 & 0 & 14 & 11 \\
\hline FormZone & 4.1 & 6.5 & 4.4 & 7.2 & 0.88 & 1.3 & 3 & 6.2 & 5 & 8.3 \\
\hline MFP_pi & 3.1 & 3.1 & 3.1 & 3.1 & 1.3 & 1.2 & 1.9 & 2 & 3.1 & 3.2 \\
\hline FrAbs_pi & 1.9 & 1.9 & 2.2 & 2.2 & 0.25 & 0.25 & 1.7 & 1.7 & 2.6 & 2.6 \\
\hline Calibration & 18.5 & 21.7 & 2.0 & 0.9 & 1.8 & 0.8 & 32.6 & 19.4 & 18.3 & 15.6 \\
\hline Calibration Shape & 8.4 & - & 0.5 & - & 0.4 & - & 14.4 & - & 7.3 & - \\
\hline Light Level & 3.2 & 7.6 & 0.7 & 1.4 & 0.7 & 1.3 & 9.4 & 14.7 & 3.1 & 6.6 \\
\hline Cherenkov & 4.2 & - & 1.0 & - & 1.0 & - & 12.6 & - & 5.4 & - \\
\hline summedsmallgenie & 10.6 & 10.6 & 10.0 & 12.6 & 11.1 & 11.1 & 11.3 & 11.3 & 11.7 & 11.7 \\
\hline beamTransportComb & 4.7 & 4.7 & 4.3 & 4.8 & 4.6 & 4.6 & 2.7 & 3.7 & 5.5 & 5.6 \\
\hline ppfx_pc00 & 0.6 & 0.6 & 0.5 & 0.5 & 0.6 & 0.6 & 1.9 & 1.9 & 0.8 & 0.8 \\
\hline ppfx_pc01 & 7.1 & 7.1 & 7.9 & 6.8 & 7.2 & 7.2 & 7.0 & 7.0 & 7.2 & 7.2 \\
\hline ppfx_pc02 & 3.2 & 3.2 & 3.1 & 3.3 & 3.3 & 3.2 & 1.8 & 1.8 & 2.1 & 2.1 \\
\hline ppfx_pc03 & 1.6 & 1.6 & 1.5 & 1.6 & 1.6 & 1.6 & 0.7 & 0.7 & 1.4 & 1.4 \\
\hline ppfx_pc04 & 3.6 & 3.6 & 3.8 & 3.5 & 3.5 & 3.5 & 3.6 & 3.6 & 3.5 & 3.5 \\
\hline
\end{tabular}

TABLE 5.4: Systematic summary of $\nu_{\mu}$ sample. The $\pm 1 \sigma$ shifts are the ratio between the $1 \sigma$ shifted and nominal un-oscillated prediction. The top seven are large GENIE parameters included as individual pulls, all other GENIE parameters included in the summed small genie. All five principal components for PPFX are shown.
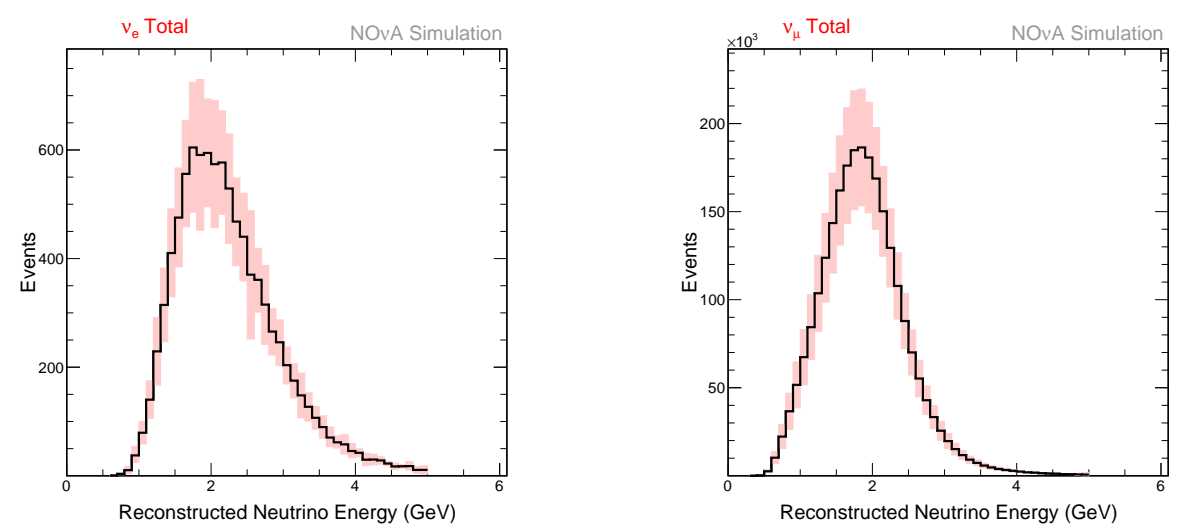

Figure 5.1: Shows effect of all systematics on $\nu_{e}$ prediction (left) and $\nu_{\mu}$ prediction (right).

\subsection{Flux Systematics}

In the third analysis, NOvA uses g4numi package for the NuMI neutrino flux simulation. Two main categories of beam uncertainties are considered: beam transport and hadron production. The g4numi package has encoded a standard geometry for the NuMI beam. For example, target and magnetic horn position, proton beam spot size, and position 


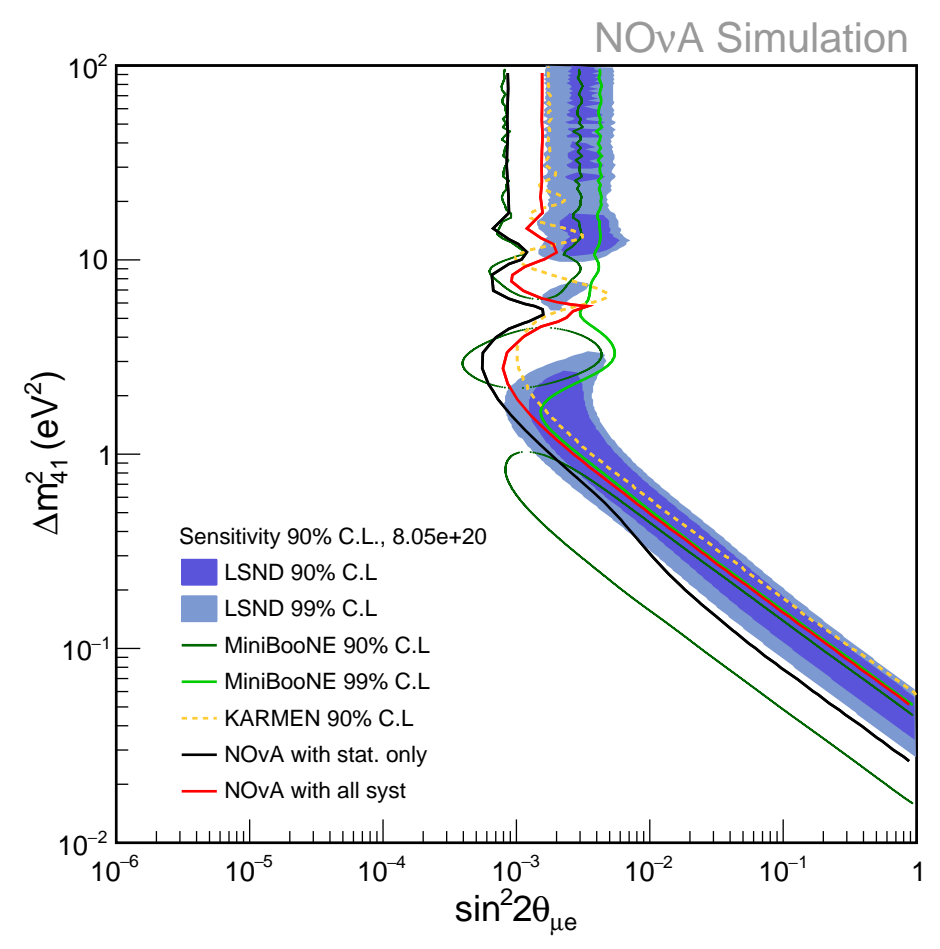

FIGURE 5.2: Effect of all systematics on $\theta_{\mu e}$ sensitivity.

of the proton beam on the carbon target. To study the systematics associated with the NuMI geometry, the effect of the difference between the simulation and the working conditions of beam, we shift each geometry parameter within an acceptable range and simulate a flux corresponding to $5 \times 10^{8}$ protons-on-target (POT) for each sample. We use the FluxReader package to obtain the shifted flux predictions. The ratio between the shifted and nominal flux predictions gives the estimate of the effect of the corresponding shift. More details on beam transport systematics can be found in Ref. [118]. The uncertainty due to all beam transport parameters is added in quadrature and given in Fig. 5.3.

The second beam uncertainty arises from the hadron production, which refers to the uncertainty in our simulation of the production of pions and kaons on the carbon target. For the third analysis, we follow a different approach to estimate this effect than had been used previously. For every produced neutrino, there is an associated weight calculated by considering the neutrino parent interaction chain and yield corrections based on the external data. This weight is saved in CAF files. This is implemented in a package called Package to Predict FluX (PPFX). To estimate the hadron production uncertainty, we use a Principal Component Analysis (PCA) method as documented in detail in Ref. [119].

It was determined by the $\nu_{e}$ group that five principal components will cover the hadron production uncertainty. These are shown together in Fig. 5.5. The effects of the above 
beam uncertainties on the sensitivity to $\theta_{\mu e}$ are shown in Fig. 5.6.
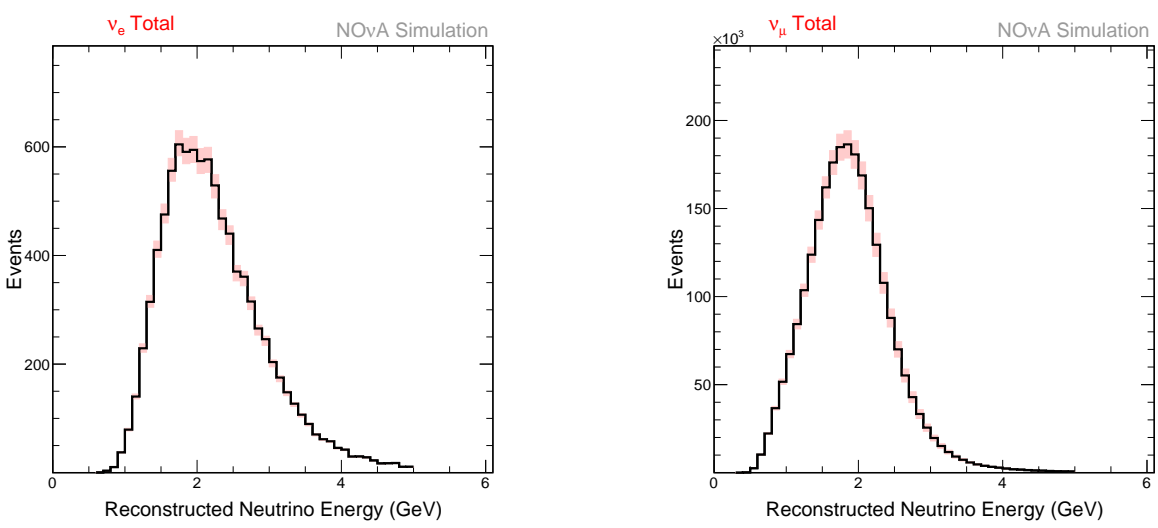

Figure 5.3: Shows the $\nu_{e}$ prediction (left) and $\nu_{\mu}$ prediction (right) with beam transport systematics.
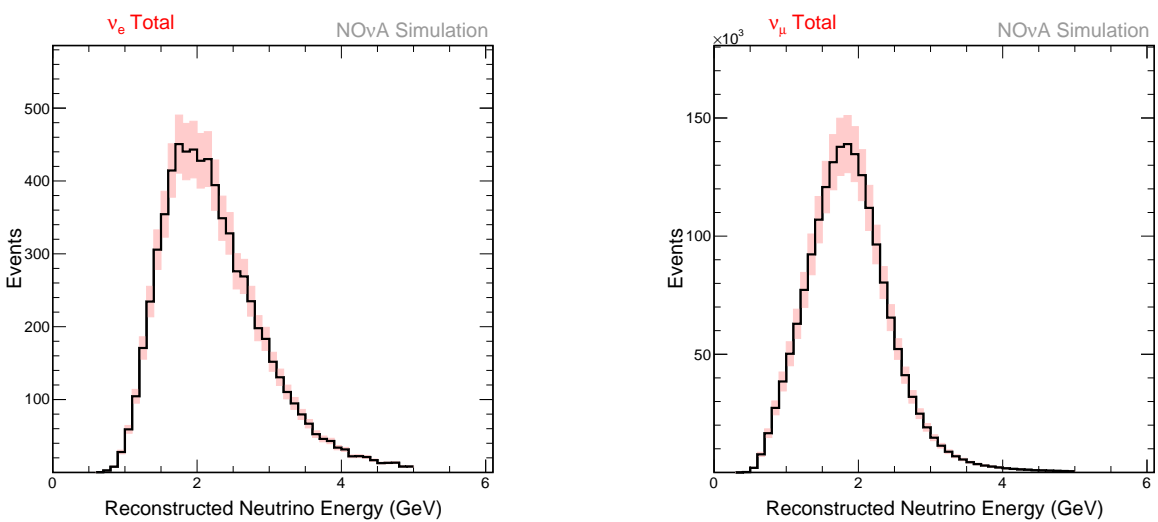

Figure 5.4: Shows the $\nu_{e}$ prediction (left) and $\nu_{\mu}$ prediction (right) with PPFX systematics with five principal components.

\subsection{Cross-section Model Systematics}

Neutrino interaction systematics are evaluated by using the reweighing tools built into GENIE. These tools compute a weighting factor that can be applied to simulated events to enhance or suppress a particular type of interaction. The effects considered fall roughly into three categories: cross-section uncertainties, hadronization model uncertainties, and uncertainties due to final state interactions. Additionally, new errors are included to account for uncertainty on the meson exchange current (MEC) processes, newly included in the simulation, and for random phase approximation (RPA); which is included to model internuclear effects. The deep-inelastic scattering (DIS) uncertainties are also included based on short track studies from the $\nu_{\mu}$ group [120]. The weights for every neutrino interaction for every flavor are saved in CAF files for \pm 1 

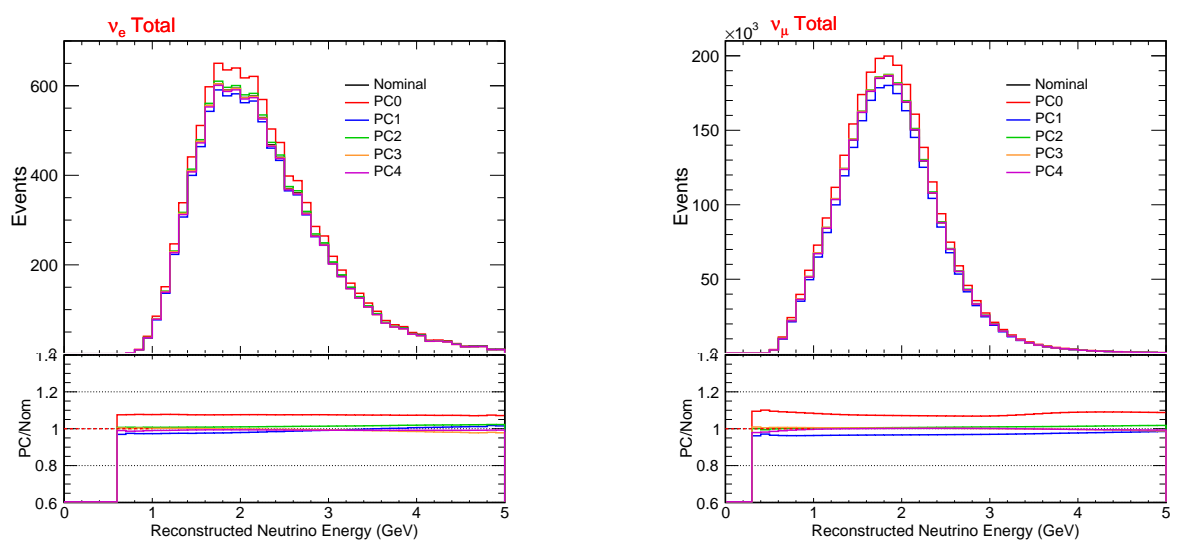

Figure 5.5: Shows the shifted $\nu_{e}$ prediction (left) and $\nu_{\mu}$ prediction (right) with ND specific PPFX systematics with five principal components.
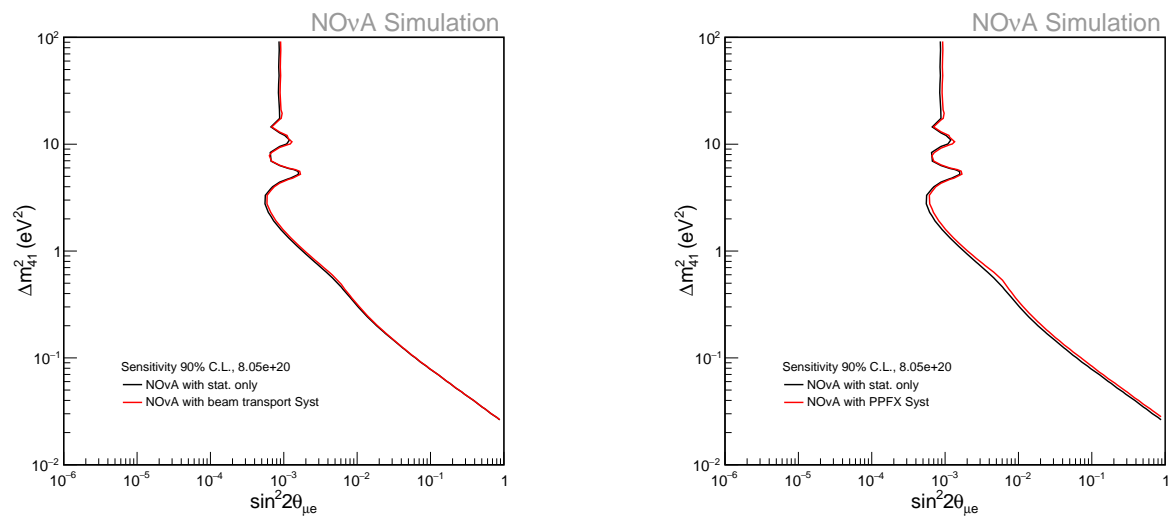

Figure 5.6: Effect of beam transport (left) and PPFX (right) systematics on $\theta_{\mu e}$ sensitivity.

sigma and \pm 2 sigma shifts. Later, these weights are used to get the shifted predictions, and the ratio between shifted and nominal gives the cross-section uncertainty corresponding to the parameter under interrogation. This analysis used the standard NOvA 2017 tune and corresponding uncertainties. We include all GENIE shifts listed in CAFAna/Systs/XSECSystLists.CXx, and we include the additional MEC, RPA and DIS uncertainties.

We estimate the uncertainty for every parameter by taking the ratio between the $\nu_{e}\left(\nu_{\mu}\right)$ selected spectrum by applying the weight for every systematic parameter saved in CAF files and the nominal selected spectrum. The uncertainty on the $\nu_{e}\left(\nu_{\mu}\right)$ selected spectrum is given in Table ?? (??) in Appendix $\mathrm{C}$ and, also shown the ratio between the nominal and $\pm 1 \%, \pm 2 \%$ shifted spectra. For this analysis, we report the parameters that contribute more than a $1 \%$ effect, as shown in Table. 5.5 and 5.6 for $\nu_{e}$ and $\nu_{\mu}$, respectively. If the systematic is considered large, it is treated as an individual pull in the fit. The systematics from the other small parameters is added in quadrature and 
used together in the fit. But, it should be noted that we only consider parameters that have an effect larger than $2 \%$ in the fit.

For the cross-section systematics, anything which has a greater than $2 \%$ effect on the total event rate is treated as an individual pull and these are listed in Tables 5.3 and 5.4. All shifts with smaller effects are summed into a total small_gennie systematic. You will note that even though the cross-section systematics are treated as correlated, only DISvnCC1pi, MaCCRES, and MvCCRES are large pulls for both the $\nu_{e}$ and the $\nu_{\mu}$ selections, so only these and the small_gennie systematic are truly treated as correlated. This means the other large pulls which only are large for one example are treated as uncorrelated. For the $\nu_{\mu}$ sample this is DISvpCC2pi, DISvpNC2pi, DISvpNC3pi, DISvnCC2pi, DISvnNC1pi, DISvnNC2pi, MaNCRES, FormZone, MFP_pi, and FrAbs_pi. For the $\nu_{e}$ sample this is RPAShapeenh2017, MECq0Shape, MECEnuShape, MaCOHpi, ROCOHpi, and MFP_N.

\begin{tabular}{|c|cc|cc|cc|cc|cc|}
\hline \multirow{2}{*}{ Syst.Parameter } & \multicolumn{2}{|c|}{$\nu_{e}$ sig } & \multicolumn{2}{|c|}{ Total bkg } & \multicolumn{2}{c|}{$\nu_{\mu}$} & \multicolumn{2}{c|}{ beam $\nu_{e}$} & \multicolumn{2}{c|}{$\mathrm{NC}$} \\
\cline { 2 - 16 } & $+1 \sigma(\%)$ & $-1 \sigma(\%)$ & $+1 \sigma(\%)$ & $-1 \sigma(\%)$ & $+1 \sigma(\%)$ & $-1 \sigma(\%)$ & $+1 \sigma(\%)$ & $-1 \sigma(\%)$ & $+1 \sigma(\%)$ & $-1 \sigma(\%)$ \\
\hline MaCCQE_reduced & 1.6 & 1.7 & 1.2 & 1.2 & 0.097 & 0.086 & 1.6 & 1.7 & 0 & 0 \\
\hline RPAShapeenh2017 & 5.5 & 4.6 & 4.5 & 3.6 & 3.2 & 2.2 & 5.5 & 4.5 & 0 & 0 \\
\hline RPAShapesupp2017 & 1.9 & 1.9 & 1.4 & 1.4 & 0.2 & 0.2 & 1.9 & 1.9 & 0 & 0 \\
\hline MECq0Shape & 9.4 & 7.2 & 6.3 & 5.7 & 0.12 & 0.073 & 8.5 & 7.8 & 0.24 & 0.24 \\
\hline MECEnuShape & 4.1 & 4.1 & 2.7 & 2.7 & 0.092 & 0.092 & 3.6 & 3.6 & 0.0069 & 0.0069 \\
\hline DISvpCC2pi & 0.63 & 0.63 & 1.2 & 1.2 & 5 & 5 & 0.74 & 0.74 & 0 & 0 \\
\hline DISvnCC1pi & 0.95 & 0.95 & 3 & 3 & 16 & 16 & 1.2 & 1.2 & 0 & 0 \\
\hline DISvnCC2pi & 0.33 & 0.33 & 1.5 & 1.5 & 8.3 & 8.3 & 0.51 & 0.51 & 0 & 0 \\
\hline DISvnNC2pi & 0 & 0 & 1 & 1 & 0 & 0 & 0 & 0 & 7.7 & 7.7 \\
\hline radcorrnue & 2 & 2 & 1.4 & 1.4 & 0 & 0 & 1.9 & 1.9 & 0 & 0 \\
\hline 2ndclasscurr & 1.9 & 1.9 & 1.3 & 1.3 & 0 & 0 & 1.7 & 1.7 & 0 & 0 \\
\hline MaCCRES & 6.5 & 7.1 & 6.1 & 6.1 & 7.1 & 6.2 & 7 & 7.2 & 0 & 0 \\
\hline MvCCRES & 3.6 & 3.4 & 3.3 & 3 & 4 & 3.5 & 3.8 & 3.4 & 0 & 0 \\
\hline MaCOHpi & 0.81 & 0.85 & 2.3 & 2.4 & 0.081 & 0.081 & 0.83 & 0.85 & 13 & 13 \\
\hline R0COHpi & 0.84 & 0.81 & 2.4 & 2.3 & 0.081 & 0.081 & 0.84 & 0.82 & 13 & 13 \\
\hline FormZone & 0.26 & 0.37 & 1.8 & 3.4 & 6.7 & 13 & 0.41 & 0.55 & 4.7 & 10 \\
\hline MFP_pi & 0.76 & 0.67 & 1 & 1.1 & 2 & 2.5 & 0.73 & 0.68 & 1.8 & 2.2 \\
\hline MFP_N & 2.6 & 2.6 & 2 & 2.1 & 1.1 & 1.3 & 2.5 & 2.6 & 0.27 & 0.31 \\
\hline CCQEPauliSupViaKF & 1.7 & 1.4 & 1.3 & 1.1 & 0.041 & 0.02 & 1.7 & 1.5 & 0 & 0 \\
\hline
\end{tabular}

TABLE 5.5: Shows the $\pm 1 \sigma \%$ shifts for the large GENIE systematic parameters having more $1 \%$ effect on the $\nu_{e}$ selected sample. Only the parameters having larger than $2 \%$ effect are included in the fit. 


\begin{tabular}{|c|cc|cc|cc|cc|cc|}
\hline \multirow{2}{*}{ Syst.Parameter } & \multicolumn{2}{|c|}{$\nu_{-}$sig } & \multicolumn{2}{|c|}{ Total bkg } & \multicolumn{2}{c|}{$\nu_{\mu}$} & \multicolumn{2}{c|}{ beam $\nu_{e}$} & NC \\
\cline { 2 - 12 } & $+1 \sigma(\%)$ & $-1 \sigma(\%)$ & $+1 \sigma(\%)$ & $-1 \sigma(\%)$ & $+1 \sigma(\%)$ & $-1 \sigma(\%)$ & $+1 \sigma(\%)$ & $-1 \sigma(\%)$ & $+1 \sigma(\%)$ & $-1 \sigma(\%)$ \\
\hline RPAShapeenh2017 & 3.9 & 2.1 & 2.2 & 1.2 & 4.5 & 3.7 & 3.7 & 2.5 & 0 & 0 \\
\hline DISvpCC0pi & 2.9 & 2.9 & 1.7 & 1.7 & 0.85 & 0.85 & 2.8 & 2.8 & 0 & 0 \\
\hline DISvpCC1pi & 2.3 & 2.3 & 1.3 & 1.3 & 0.47 & 0.47 & 2 & 2 & 0 & 0 \\
\hline DISvpCC2pi & 4.2 & 4.2 & 2.4 & 2.4 & 1.5 & 1.5 & 3.6 & 3.6 & 0 & 0 \\
\hline DISvpCC3pi & 3 & 3 & 1.7 & 1.7 & 0.88 & 0.88 & 3 & 3 & 0 & 0 \\
\hline DISvpNC0pi & 0 & 0 & 1.8 & 1.8 & 0 & 0 & 0 & 0 & 4.2 & 4.2 \\
\hline DISvpNC2pi & 0 & 0 & 2.7 & 2.7 & 0 & 0 & 0 & 0 & 6.4 & 6.4 \\
\hline DISvpNC3pi & 0 & 0 & 2.2 & 2.2 & 0 & 0 & 0 & 0 & 5.3 & 5.3 \\
\hline DISvnCC1pi & 5.6 & 5.6 & 3.2 & 3.2 & 2 & 2 & 4.7 & 4.7 & 0 & 0 \\
\hline DISvnCC2pi & 4.4 & 4.4 & 2.5 & 2.5 & 1.3 & 1.3 & 4.1 & 4.1 & 0 & 0 \\
\hline DISvnCC3pi & 1.7 & 1.7 & 1 & 1 & 0.44 & 0.44 & 6 & 6 & 0 & 0 \\
\hline DISvnNC1pi & 0 & 0 & 2.7 & 2.7 & 0 & 0 & 0 & 0 & 6.4 & 6.4 \\
\hline DISvnNC2pi & 0 & 0 & 2 & 2 & 0 & 0 & 0 & 0 & 4.8 & 4.8 \\
\hline DISvnNC3pi & 0 & 0 & 1.2 & 1.2 & 0 & 0 & 0 & 0 & 2.9 & 2.9 \\
\hline radcorrnue & 2 & 2 & 1.1 & 1.1 & 0 & 0 & 2 & 2 & 0 & 0 \\
\hline 2ndclasscurr & 2 & 2 & 1.1 & 1.1 & 0 & 0 & 1.9 & 1.9 & 0 & 0 \\
\hline MaCCRES & 15 & 12 & 8.3 & 7.1 & 8 & 8.6 & 10 & 9 & 0 & 0 \\
\hline MvCCRES & 8.3 & 7 & 4.8 & 4 & 4.5 & 4.2 & 5.9 & 5.1 & 0 & 0 \\
\hline MaNCRES & 0 & 0 & 5.7 & 4.5 & 0 & 0 & 0 & 0 & 14 & 11 \\
\hline MvNCRES & 0 & 0 & 1.3 & 1.1 & 0 & 0 & 0 & 0 & 3.2 & 2.7 \\
\hline FormZone & 4.1 & 6.5 & 4.4 & 7.2 & 0.88 & 1.3 & 3 & 6.2 & 5 & 8.3 \\
\hline MFP_pi & 3.1 & 3.1 & 3.1 & 3.1 & 1.3 & 1.2 & 1.9 & 2 & 3.1 & 3.2 \\
\hline MFP_N & 1.8 & 2 & 1.5 & 1.7 & 2.1 & 2.2 & 1.8 & 2 & 1 & 1.2 \\
\hline FrInel_pi & 1.6 & 1.5 & 1.8 & 1.7 & 0.096 & 0.095 & 1.5 & 1.4 & 1.9 & 1.9 \\
\hline FrAbs_pi & 1.9 & 1.9 & 2.2 & 2.2 & 0.25 & 0.25 & 1.7 & 1.7 & 2.6 & 2.6 \\
\hline
\end{tabular}

TABLE 5.6: Shows the $\pm 1 \sigma \%$ shifts for the large GENIE systematic parameters having more than $1 \%$ effect on the $\nu_{\mu}$ selected sample. 
In Fig. 5.7 and 5.8, we have shown the effect of large and small GENIE systematics (added in quadrature) on the $\nu_{e}$ and $\nu_{\mu}$ predictions. We observe that the effect is seemingly large for both $\nu_{e}$ and $\nu_{\mu}$. We show the effect of GENIE systematics on the $\theta_{\mu e}$ sensitivities for joint fit in Fig. 5.9. The effect is mainly canceled in the joint fit.
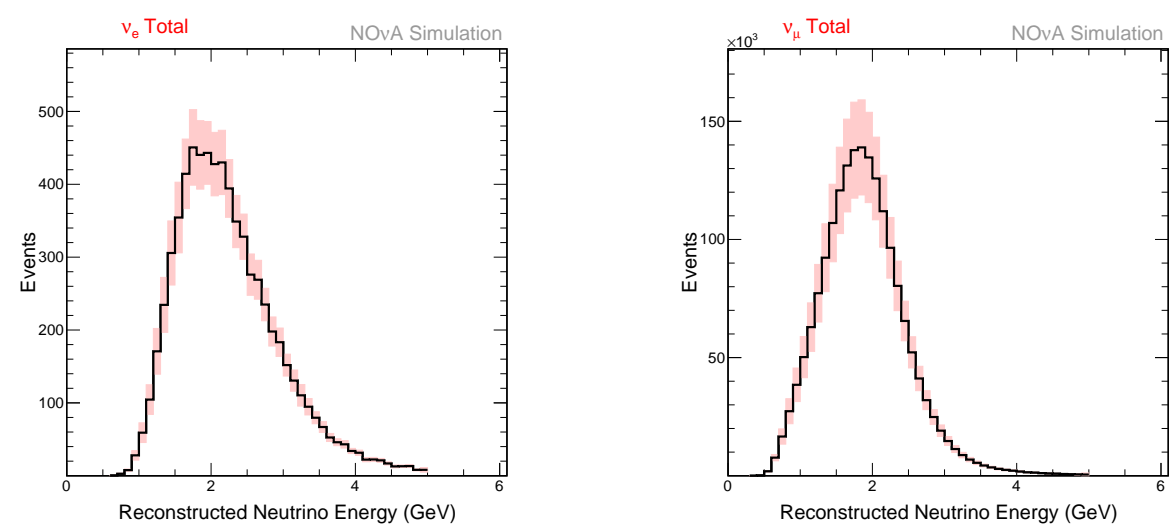

FIGURE 5.7: Showing the effect of all large cross-section systematics having more than $2 \%$ effect on both $\nu_{e}$ prediction (left) and $\nu_{\mu}$ prediction (right).
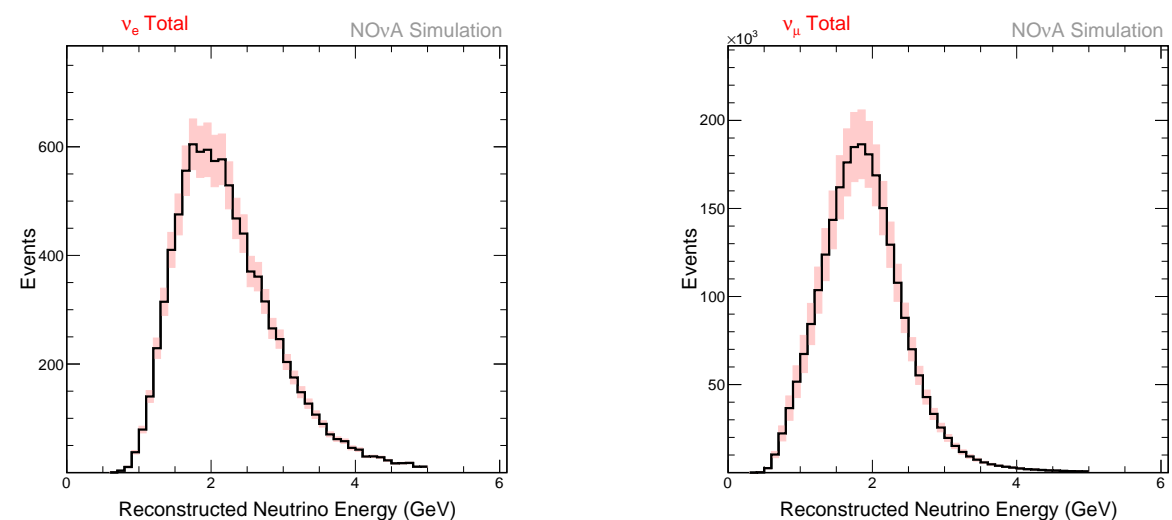

Figure 5.8: Showing the effect of summed small cross-section systematics on both $\nu_{e}$ prediction (left) and $\nu_{\mu}$ prediction (right).

\subsubsection{Neutral Currents}

The uncertainty on the Neutral Current (NC) background comes directly from the corresponding GENIE cross-section uncertainties, and there is no additional NC uncertainty as there has been for previous NOvA analysis. This is motivated by our general better understanding of the uncertainties on the $\mathrm{NC}$ and that add in an additional $\mathrm{NC}$ uncertainty is very conservative and double counting. The evidence for this comes from the studies of neutrons to find $\mathrm{NC}$ and RHC numu contamination in the $\nu_{\mu}$ analysis as documented in DocDB 22955. For the reader easy the NC Genie uncertainties are pulled out and listed in Table 5.9. These NC genie knobs have a $2 \%$ effect on the total $\nu_{e}$ selected sample $\left(16 \%\right.$ on the $\mathrm{NC}$ in the $\nu_{e}$ selected sample) and a $7 \%$ on the $\nu_{\mu}$ selected sample 

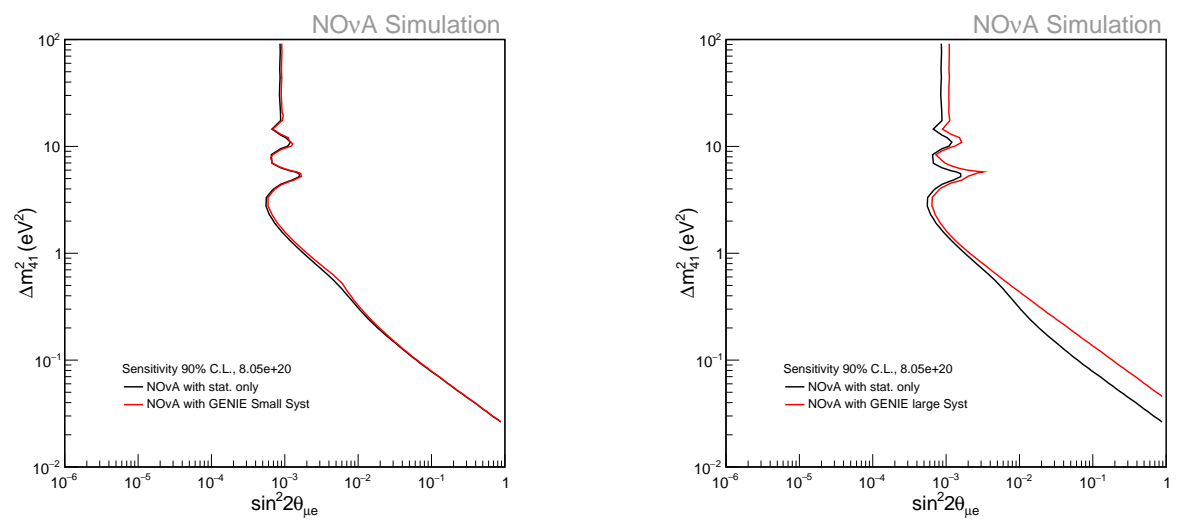

Figure 5.9: Effect of GENIE systematics on the $\theta_{\mu e}$ sensitivity at $90 \%$ C.L. using joint fit (red) as compared to black which has no systematics included. The left plot shows the effect of summed small GENIE and the right plot shows the effect of large

GENIE parameters.

(18\% on the $\mathrm{NC}$ in the $\nu_{e}$ selected sample). Of the $19 \mathrm{NC}$ knobs, five of them with the largest effect are included as uncorrelated individual pulls in the $\nu_{\mu}$ selected sample, nine are included as individual pulls for the $\nu_{e}$ selected sample. These are DISvpNC2pi, DISvpNC3pi, DISvnNC1pi, DISvnNC2pi, and MaNCRES. These 5 have a total effect of $7.4 \%$ for the $\nu_{\mu}$ selected sample.

\begin{tabular}{|c|cc|cc|cc|cc|cc|}
\hline \multirow{2}{*}{ Syst.Parameter } & \multicolumn{2}{|c|}{$\nu_{e}$ sig } & \multicolumn{2}{|c|}{ Total bkg } & \multicolumn{2}{c|}{$\nu_{\mu}$} & \multicolumn{2}{c|}{ beam $\nu_{e}$} & \multicolumn{2}{c|}{ NC } \\
\cline { 2 - 14 } & $+1 \sigma(\%)$ & $-1 \sigma(\%)$ & $+1 \sigma(\%)$ & $-1 \sigma(\%)$ & $+1 \sigma(\%)$ & $-1 \sigma(\%)$ & $+1 \sigma(\%)$ & $-1 \sigma(\%)$ & $+1 \sigma(\%)$ & $-1 \sigma(\%)$ \\
\hline DISvpNC1pi & 0 & 0 & 0.57 & 0.57 & 0 & 0 & 0 & 0 & 4.3 & 4.3 \\
\hline DISvpNC2pi & 0 & 0 & 0.87 & 0.87 & 0 & 0 & 0 & 0 & 6.5 & 6.5 \\
\hline DISvpNC3pi & 0 & 0 & 0.22 & 0.22 & 0 & 0 & 0 & 0 & 1.6 & 1.6 \\
\hline DISvnNC0pi & 0 & 0 & 0.0059 & 0.0059 & 0 & 0 & 0 & 0 & 0.044 & 0.044 \\
\hline DISvnNC1pi & 0 & 0 & 0.98 & 0.98 & 0 & 0 & 0 & 0 & 7.3 & 7.3 \\
\hline DISvnNC2pi & 0 & 0 & 1 & 1 & 0 & 0 & 0 & 0 & 7.7 & 7.7 \\
\hline DISvnNC3pi & 0 & 0 & 0.23 & 0.23 & 0 & 0 & 0 & 0 & 1.7 & 1.7 \\
\hline DISvbarpNC0pi & 0 & 0 & 0.031 & 0.031 & 0 & 0 & 0 & 0 & 0.23 & 0.23 \\
\hline DISvbarpNC1pi & 0 & 0 & 0.055 & 0.055 & 0 & 0 & 0 & 0 & 0.41 & 0.41 \\
\hline DISvbarpNC2pi & 0 & 0 & 0.046 & 0.046 & 0 & 0 & 0 & 0 & 0.35 & 0.35 \\
\hline DISvbarpNC3pi & 0 & 0 & 0.008 & 0.008 & 0 & 0 & 0 & 0 & 0.06 & 0.06 \\
\hline DISvbarnNC0pi & 0 & 0 & 0 & 0 & 0 & 0 & 0 & 0 & 0 & 0 \\
\hline DISvbarnNC1pi & 0 & 0 & 0.021 & 0.021 & 0 & 0 & 0 & 0 & 0.16 & 0.16 \\
\hline DISvbarnNC2pi & 0 & 0 & 0.053 & 0.053 & 0 & 0 & 0 & 0 & 0.39 & 0.39 \\
\hline DISvbarnNC3pi & 0 & 0 & 0.013 & 0.013 & 0 & 0 & 0 & 0 & 0.096 & 0.096 \\
\hline MaNCEL & 0 & 0 & 0.03 & 0.013 & 0 & 0 & 0 & 0 & 0.22 & 0.095 \\
\hline EtaNCEL & 0 & 0 & 0.00035 & 0.00033 & 0 & 0 & 0 & 0 & 0.0026 & 0.0025 \\
\hline MaNCRES & 0 & 0 & 1.2 & 0.92 & 0 & 0 & 0 & 0 & 9.2 & 6.9 \\
\hline MvNCRES & 0 & 0 & 0.29 & 0.24 & 0 & 0 & 0 & 0 & 2.2 & 1.8 \\
\hline
\end{tabular}

TABLE 5.7: Effect of NC GENIE parameters on the $\nu_{e}$ selected sample.

The effect of GENIE parameters on $\nu_{e}$ and $\nu_{\mu}$ selected sample which has larger than $2 \%$ either on total selected or on appeared $\nu_{e}\left(\nu_{\mu}\right.$ survived) are shown below. 


\begin{tabular}{|c|cc|cc|cc|cc|cc|}
\hline \multirow{2}{*}{ Syst.Parameter } & \multicolumn{2}{|c|}{$\nu_{e}$ sig } & \multicolumn{2}{|c|}{ Total bkg } & \multicolumn{2}{c|}{$\nu_{\mu}$} & \multicolumn{2}{c|}{ beam $\nu_{e}$} & \multicolumn{2}{c|}{ NC } \\
\cline { 2 - 13 } & $+1 \sigma(\%)$ & $-1 \sigma(\%)$ & $+1 \sigma(\%)$ & $-1 \sigma(\%)$ & $+1 \sigma(\%)$ & $-1 \sigma(\%)$ & $+1 \sigma(\%)$ & $-1 \sigma(\%)$ & $+1 \sigma(\%)$ & $-1 \sigma(\%)$ \\
\hline DISvpNC1pi & 0 & 0 & 0.84 & 0.84 & 0 & 0 & 0 & 0 & 2 & 2 \\
\hline DISvpNC2pi & 0 & 0 & 2.7 & 2.7 & 0 & 0 & 0 & 0 & 6.4 & 6.4 \\
\hline DISvpNC3pi & 0 & 0 & 2.2 & 2.2 & 0 & 0 & 0 & 0 & 5.3 & 5.3 \\
\hline DISvnNC0pi & 0 & 0 & 0.21 & 0.21 & 0 & 0 & 0 & 0 & 0.5 & 0.5 \\
\hline DISvnNC1pi & 0 & 0 & 2.7 & 2.7 & 0 & 0 & 0 & 0 & 6.4 & 6.4 \\
\hline DISvnNC2pi & 0 & 0 & 2 & 2 & 0 & 0 & 0 & 0 & 4.8 & 4.8 \\
\hline DISvnNC3pi & 0 & 0 & 1.2 & 1.2 & 0 & 0 & 0 & 0 & 2.9 & 2.9 \\
\hline DISvbarpNC0pi & 0 & 0 & 0.086 & 0.086 & 0 & 0 & 0 & 0 & 0.21 & 0.21 \\
\hline DISvbarpNC1pi & 0 & 0 & 0.11 & 0.11 & 0 & 0 & 0 & 0 & 0.26 & 0.26 \\
\hline DISvbarpNC2pi & 0 & 0 & 0.093 & 0.093 & 0 & 0 & 0 & 0 & 0.22 & 0.22 \\
\hline DISvbarpNC3pi & 0 & 0 & 0.067 & 0.067 & 0 & 0 & 0 & 0 & 0.16 & 0.16 \\
\hline DISvbarnNC0pi & 0 & 0 & 0.011 & 0.011 & 0 & 0 & 0 & 0 & 0.026 & 0.026 \\
\hline DISvbarnNC1pi & 0 & 0 & 0.077 & 0.077 & 0 & 0 & 0 & 0 & 0.18 & 0.18 \\
\hline DISvbarnNC2pi & 0 & 0 & 0.12 & 0.12 & 0 & 0 & 0 & 0 & 0.27 & 0.27 \\
\hline DISvbarnNC3pi & 0 & 0 & 0.099 & 0.099 & 0 & 0 & 0 & 0 & 0.23 & 0.23 \\
\hline MaNCEL & 0 & 0 & 0.5 & 0.23 & 0 & 0 & 0 & 0 & 1.2 & 0.56 \\
\hline EtaNCEL & 0 & 0 & 0.004 & 0.0036 & 0 & 0 & 0 & 0 & 0.0095 & 0.0085 \\
\hline MaNCRES & 0 & 0 & 5.7 & 4.5 & 0 & 0 & 0 & 0 & 14 & 11 \\
\hline MvNCRES & 0 & 0 & 1.3 & 1.1 & 0 & 0 & 0 & 0 & 3.2 & 2.7 \\
\hline
\end{tabular}

TABLE 5.8: Effect of NC GENIE parameters on the $\nu_{\mu}$ selected sample.

\begin{tabular}{|c|c|c|c|c|c|c|c|c|c|c|c|c|}
\hline \multirow{2}{*}{ Syst.Parameter } & \multicolumn{2}{|c|}{$\nu_{e} \mathrm{NC}$} & \multicolumn{2}{|c|}{$\nu_{\mu} \mathrm{NC}$} & \multicolumn{2}{|c|}{ Diff. } & \multicolumn{2}{|c|}{$\nu_{e}$ Total } & \multicolumn{2}{|c|}{$\nu_{\mu}$ Total } & \multicolumn{2}{|c|}{ Diff. } \\
\hline & $+1 \sigma(\%)$ & $-1 \sigma(\%)$ & $+1 \sigma(\%)$ & $-1 \sigma(\%)$ & $+1 \sigma(\%)$ & $-1 \sigma(\%)$ & $+1 \sigma(\%)$ & $-1 \sigma(\%)$ & $+1 \sigma(\%)$ & $-1 \sigma(\%)$ & $+1 \sigma(\%)$ & $-1 \sigma(\%)$ \\
\hline DISvpNC2pi & 7 & 7 & 6 & 6 & 0.1 & 2 & 0.9 & 0.9 & 3 & 3 & 2 & 2 \\
\hline DISvnNC0pi & 0.04 & 0.04 & 0.5 & 0.5 & 0.5 & 0.2 & 0.006 & 0.006 & 0.2 & 0.2 & 0.2 & 0.2 \\
\hline DISvnNC1pi & 7 & 7 & 6 & 6 & 0.9 & 2 & 1 & 1 & 3 & 3 & 2 & 2 \\
\hline DISvnNC2pi & 8 & 8 & 5 & 5 & 3 & 1 & 1 & 1 & 2 & 2 & 1 & 1 \\
\hline DISvbarpNC0pi & 0.2 & 0.2 & 0.2 & 0.2 & 0.02 & 0.06 & 0.03 & 0.03 & 0.09 & 0.09 & 0.06 & 0.06 \\
\hline DISvbarpNC1pi & 0.4 & 0.4 & 0.3 & 0.3 & 0.2 & 0.05 & 0.06 & 0.06 & 0.1 & 0.1 & 0.05 & 0.05 \\
\hline DISvbarpNC2pi & 0.3 & 0.3 & 0.2 & 0.2 & 0.1 & 0.05 & 0.05 & 0.05 & 0.09 & 0.09 & 0.05 & 0.05 \\
\hline DISvbarpNC3pi & 0.06 & 0.06 & 0.2 & 0.2 & 0.1 & 0.06 & 0.008 & 0.008 & 0.07 & 0.07 & 0.06 & 0.06 \\
\hline DISvbarnNC0pi & 0 & 0 & 0.03 & 0.03 & 0.03 & 0.01 & 0 & 0 & 0.01 & 0.01 & 0.01 & 0.01 \\
\hline DISvbarnNC1pi & 0.2 & 0.2 & 0.2 & 0.2 & 0.03 & 0.06 & 0.02 & 0.02 & 0.08 & 0.08 & 0.06 & 0.06 \\
\hline MaNCRES & 9 & 7 & $1 \mathrm{e}+01$ & $1 e+01$ & 4 & 4 & 1 & 0.9 & 6 & 4 & 4 & 4 \\
\hline MvNCRES & 2 & 2 & 3 & 3 & 1 & 0.9 & 0.3 & 0.2 & 1 & 1 & 1 & 0.9 \\
\hline Total (Quad Error) & 16 & 15 & 18 & 16 & 7.1 & 6.7 & 2.2 & 2 & 7.7 & 6.9 & 5.8 & 5.1 \\
\hline
\end{tabular}

TABLE 5.9: GENIE NC systematic parameters.

\subsection{Calibration Systematics}

Some uncertainty is associated with the determined calibration constants which contribute to total systematics. We estimate the effect of calibration by making samples using the mis-calibrated constants. We study the three types of calibration shifted samples, namely positive offset, negative offset and shape variation. The shifted positive and negative offset samples represent uncertainty in the calorimetric energy scale conversion (PECorr $\rightarrow \mathrm{MeV})$. It is set to $\pm 5 \%$ where plus refers to positive offset, and negative refers to negative offset. The effect of calibration offset on the $\nu_{e}$ and $\nu_{\mu}$ predictions are shown in Fig. 5.23 and the effect on the $\theta_{\mu e}$ sensitivity is shown in Fig. ??. The 

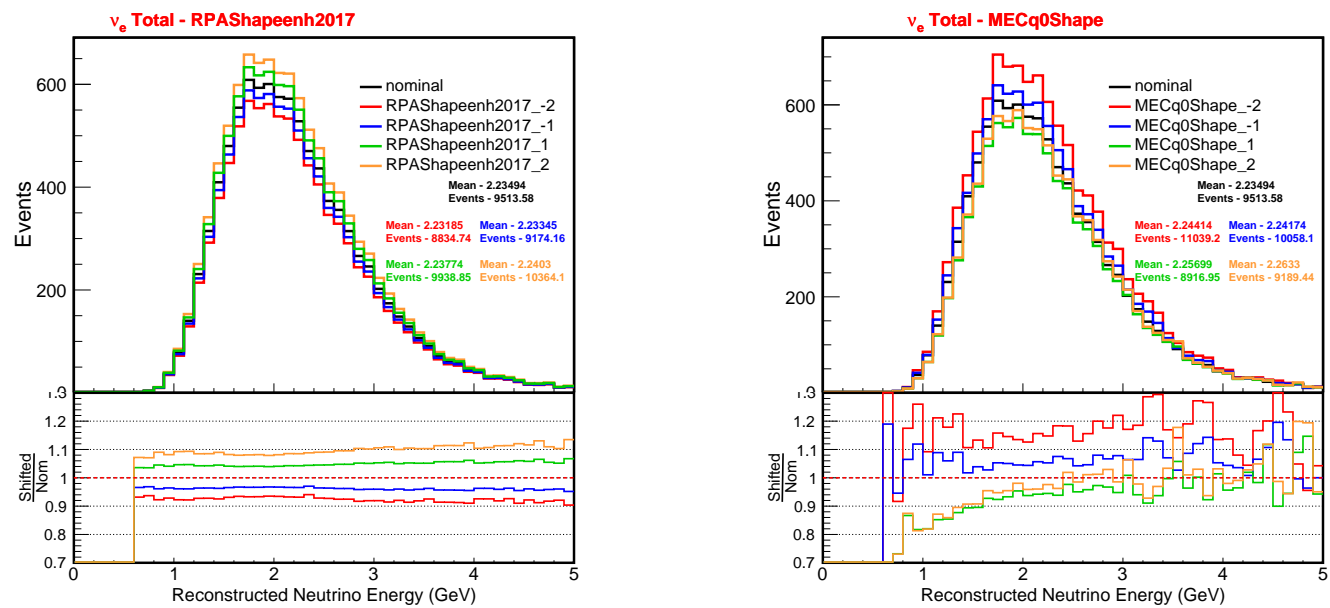

Figure 5.10: Shows the effect of GENIE parameter RPAShapeenh2017 (left) and MECq0Shape (right) $\nu_{e}$ selected sample which doesn't include appeared $\nu_{e}$ 's. The effect of large genie parameters on the both $\nu_{e}$ and $\nu_{\mu}$ selected spectra is shown in

Table 5.3 and 5.4 .
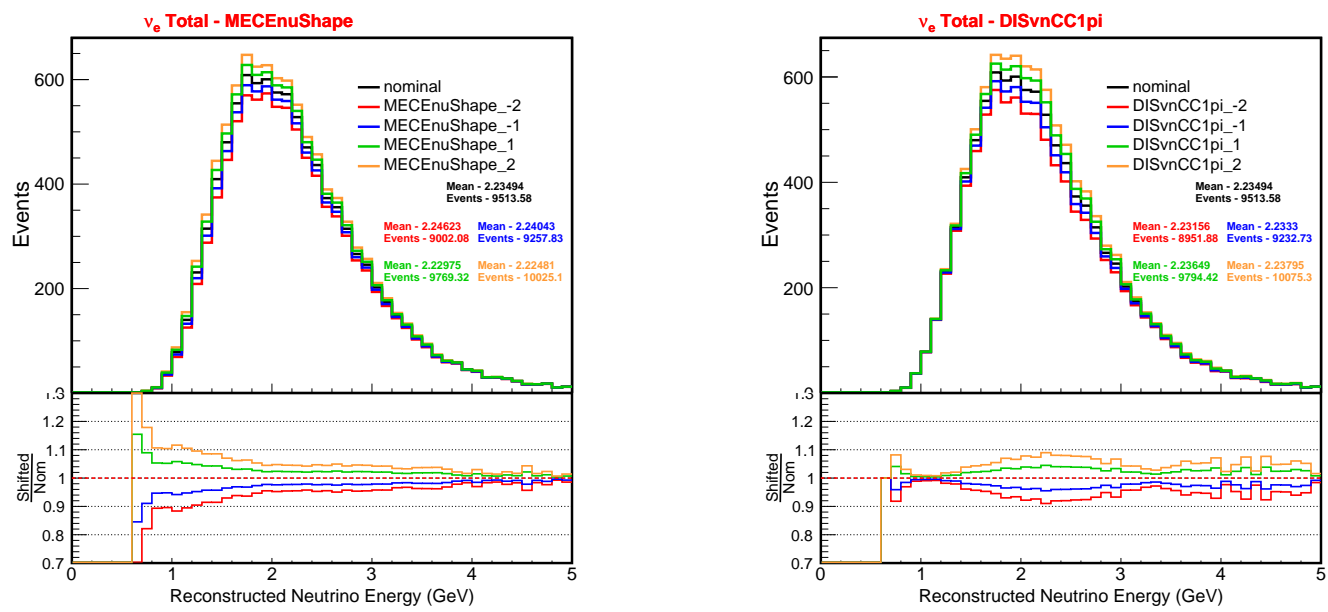

Figure 5.11: Shows the effect of GENIE parameter MECEnuShape (left) and DISvnCC1pi (right) $\nu_{e}$ selected sample which doesn't include appeared $\nu_{e}$ 's. The effect of large genie parameters on the both $\nu_{e}$ and $\nu_{\mu}$ selected spectra is shown in Table 5.3 and 5.4.

calibration shape variation represents the uncertainty in attenuation and threshold corrections (PE $\rightarrow$ PECorr). A polynomial functional form is applied across the length of a cell which is the same for one view. The calibration shape variation on the $\nu_{e}$ and $\nu_{\mu}$ predictions are shown in Fig. 5.24 and the effect of this systematic uncertainty on the $\theta_{\mu e}$ limits is shown in Fig. ??. 

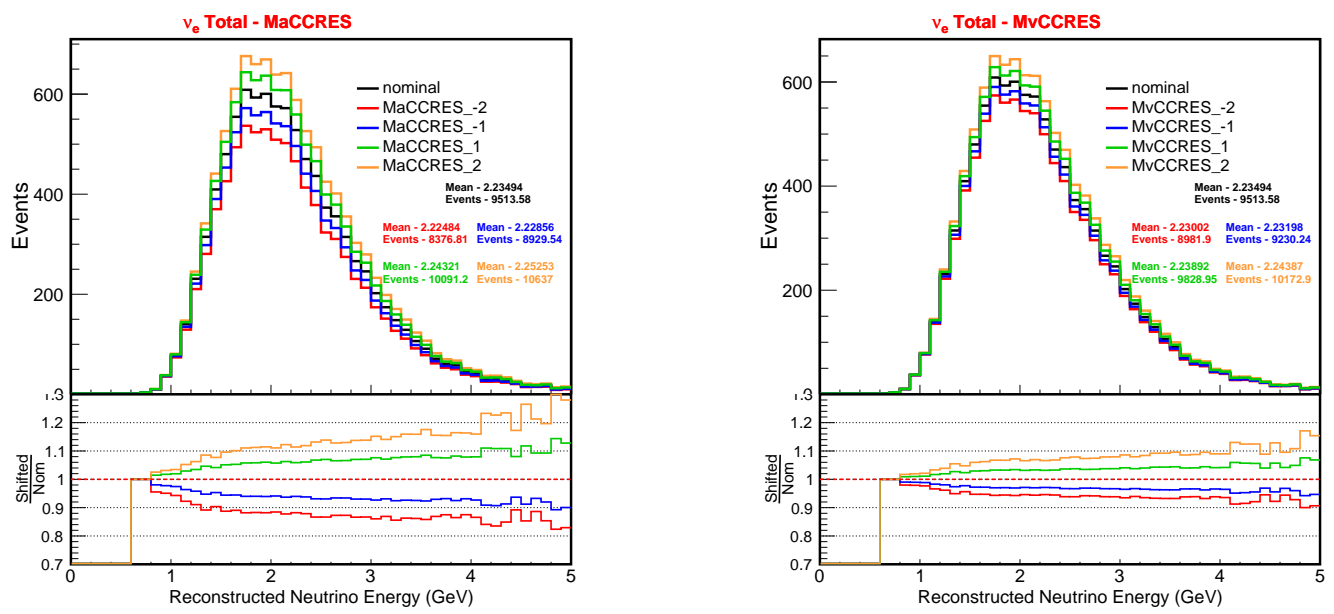

FIGURE 5.12: Shows the effect of GENIE parameter MaCCRES (left) and MvCCRES (right) $\nu_{e}$ selected sample which doesn't include appeared $\nu_{e}$ 's. The effect of large genie parameters on the both $\nu_{e}$ and $\nu_{\mu}$ selected spectra is shown in Table 5.3 and 5.4.
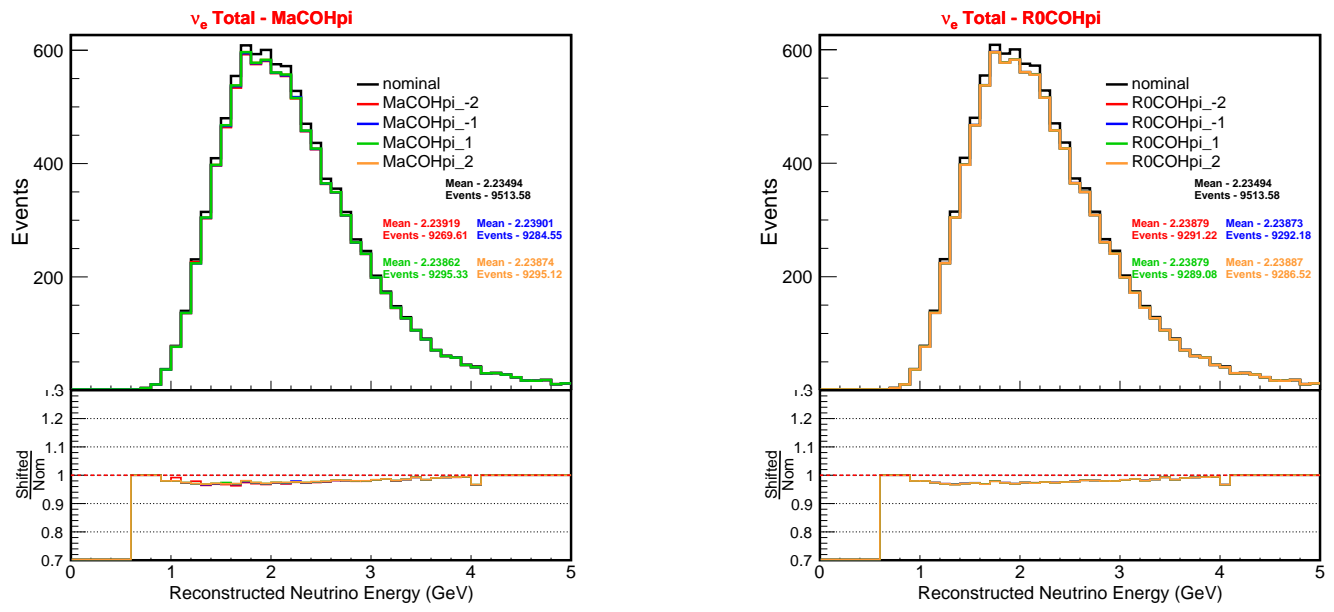

Figure 5.13: Shows the effect of GENIE parameter MaCOHpi (left) and R0COHpi (right) $\nu_{e}$ selected sample which doesn't include appeared $\nu_{e}$ 's. The effect of large genie parameters on the both $\nu_{e}$ and $\nu_{\mu}$ selected spectra is shown in Table 5.3 and 5.4. 


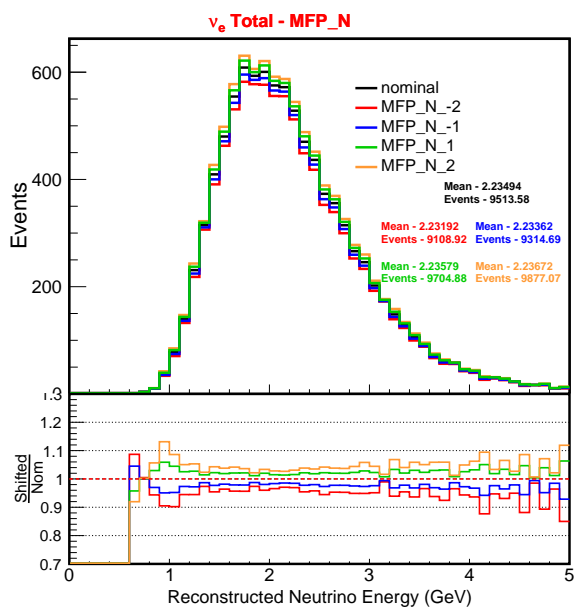

FiguRE 5.14: Shows the effect of GENIE parameter MFP_N on $\nu_{e}$ selected sample which doesn't include appeared $\nu_{e}$ 's. The effect of large genie parameters on the both $\nu_{e}$ and $\nu_{\mu}$ selected spectra is shown in Table 5.3 and 5.4.
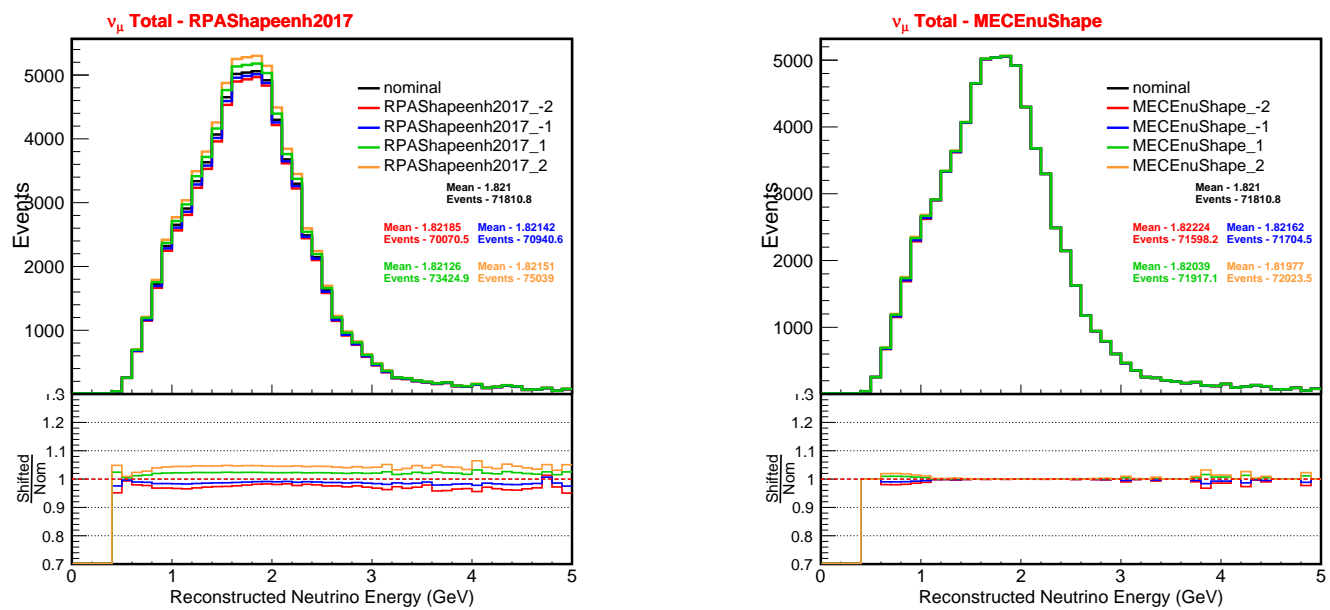

FiguRE 5.15: Shows the effect of GENIE parameter RPAShapeenh2017 (left) and MECEnuShape (right) $\nu_{\mu}$ selected sample which doesn't include appeared $\nu_{e}$ 's. The effect of large genie parameters on the both $\nu_{e}$ and $\nu_{\mu}$ selected spectra is shown in Table 5.3 and 5.4 . 

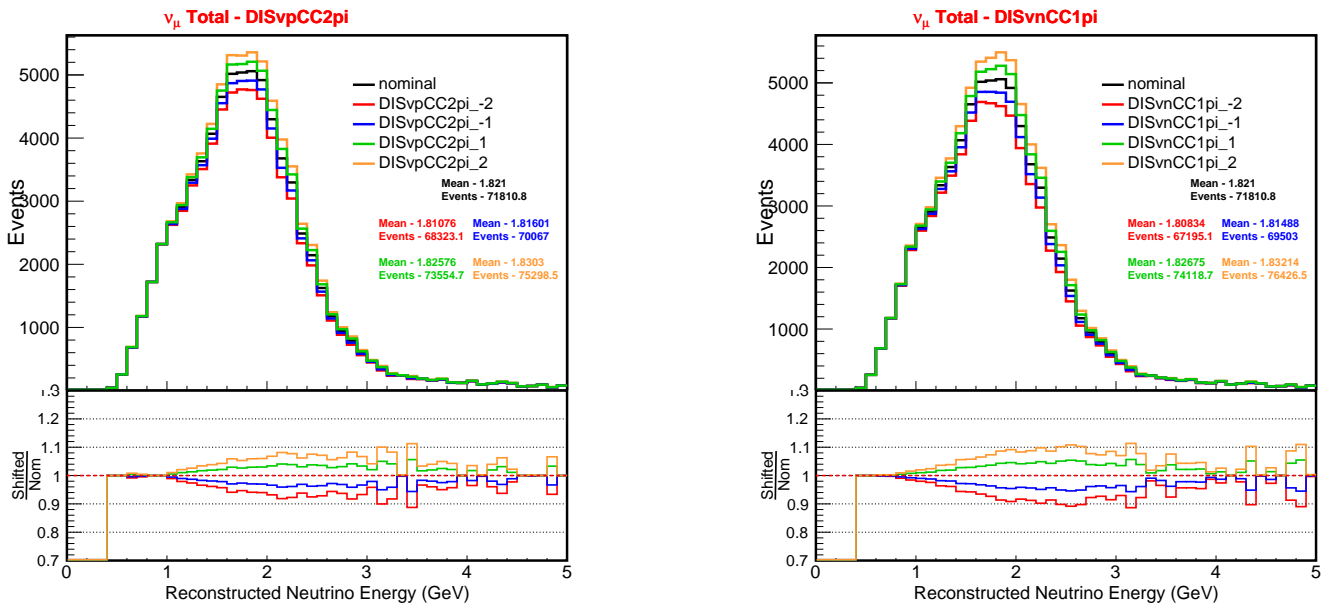

FIGURE 5.16: Shows the effect of GENIE parameter DISvpCC2pi (left) and DISvnCC1pi (right) on $\nu_{\mu}$ selected sample which doesn't include appeared $\nu_{e}$ 's. The effect of large genie parameters on the both $\nu_{e}$ and $\nu_{\mu}$ selected spectra is shown in Table 5.3 and 5.4.
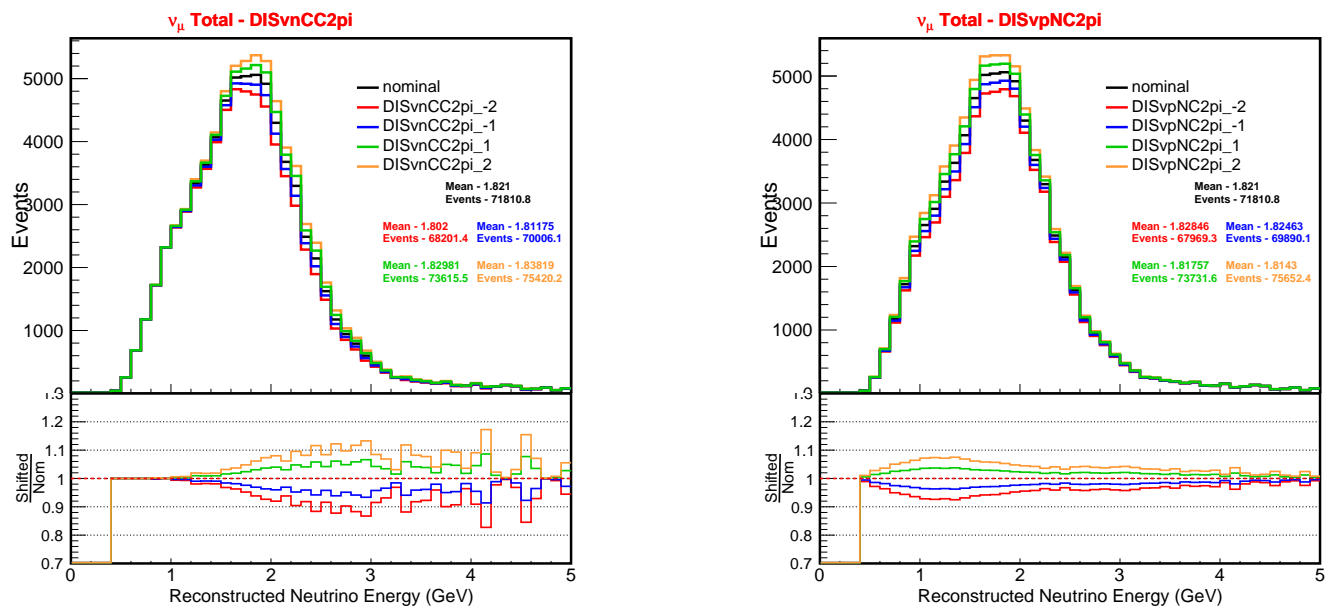

Figure 5.17: Shows the effect of GENIE parameter DISvnCC2pi (left) and DISvpNC2pi (right) on $\nu_{\mu}$ selected sample which doesn't include appeared $\nu_{e}$ 's. The effect of large genie parameters on the both $\nu_{e}$ and $\nu_{\mu}$ selected spectra is shown in Table 5.3 and 5.4. 

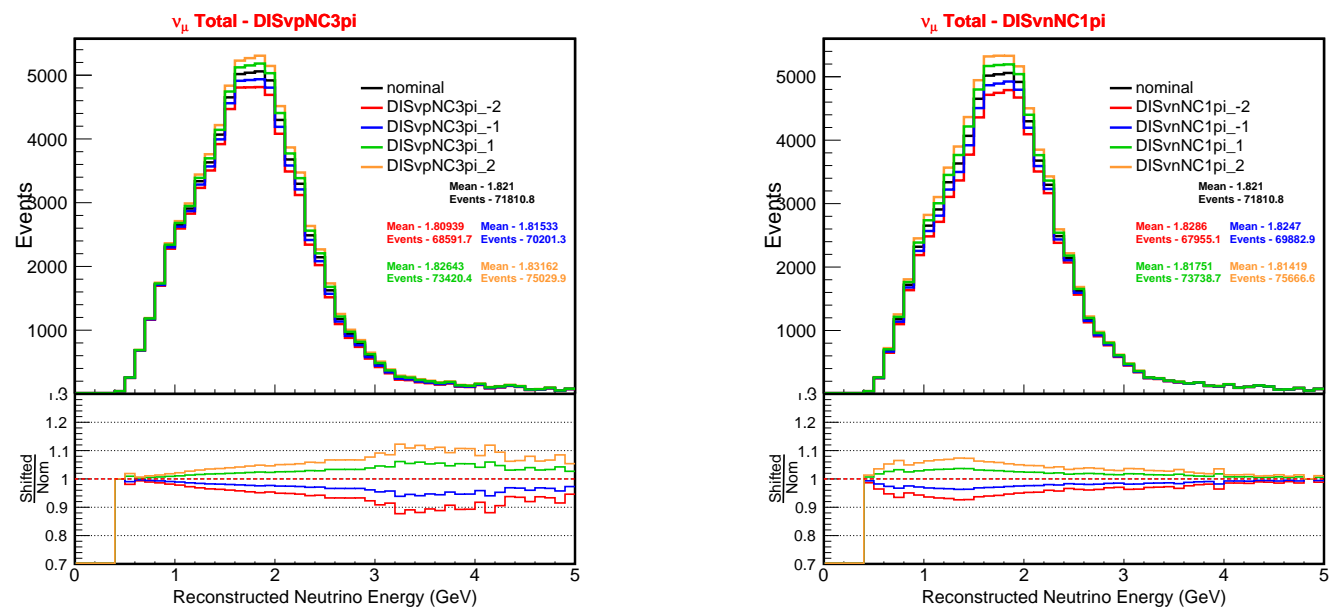

FiguRE 5.18: Shows the effect of GENIE parameter DISvpNC3pi (left) and DISvnNC1pi (right) on $\nu_{\mu}$ selected sample which doesn't include appeared $\nu_{e}$ 's. The effect of large genie parameters on the both $\nu_{e}$ and $\nu_{\mu}$ selected spectra is shown in Table 5.3 and 5.4.
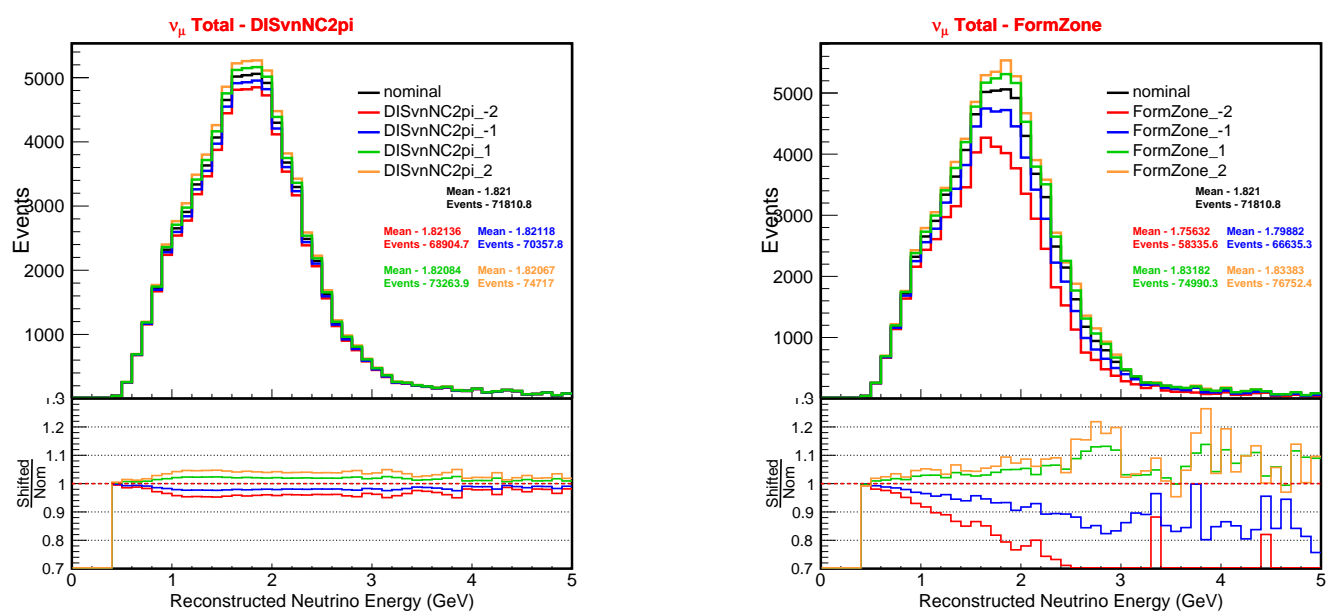

FiguRE 5.19: Shows the effect of GENIE parameter DISvnNC2pi (left) and FormZone (right) on $\nu_{\mu}$ selected sample which doesn't include appeared $\nu_{e}$ 's. The effect of large genie parameters on the both $\nu_{e}$ and $\nu_{\mu}$ selected spectra is shown in Table 5.3 and 5.4. 

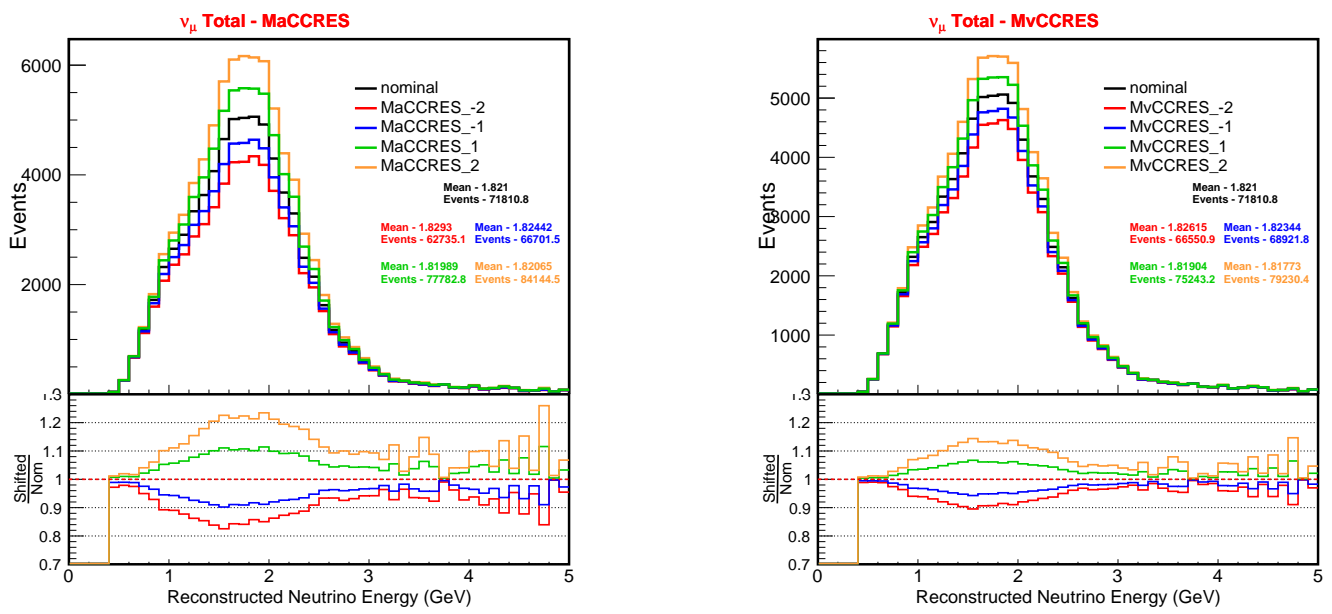

FIGURE 5.20: Shows the effect of GENIE parameter MaCCRES (left) and MvCCRES (right) on $\nu_{\mu}$ selected sample which doesn't include appeared $\nu_{e}$ 's. The effect of large genie parameters on the both $\nu_{e}$ and $\nu_{\mu}$ selected spectra is shown in Table 5.3 and 5.4.
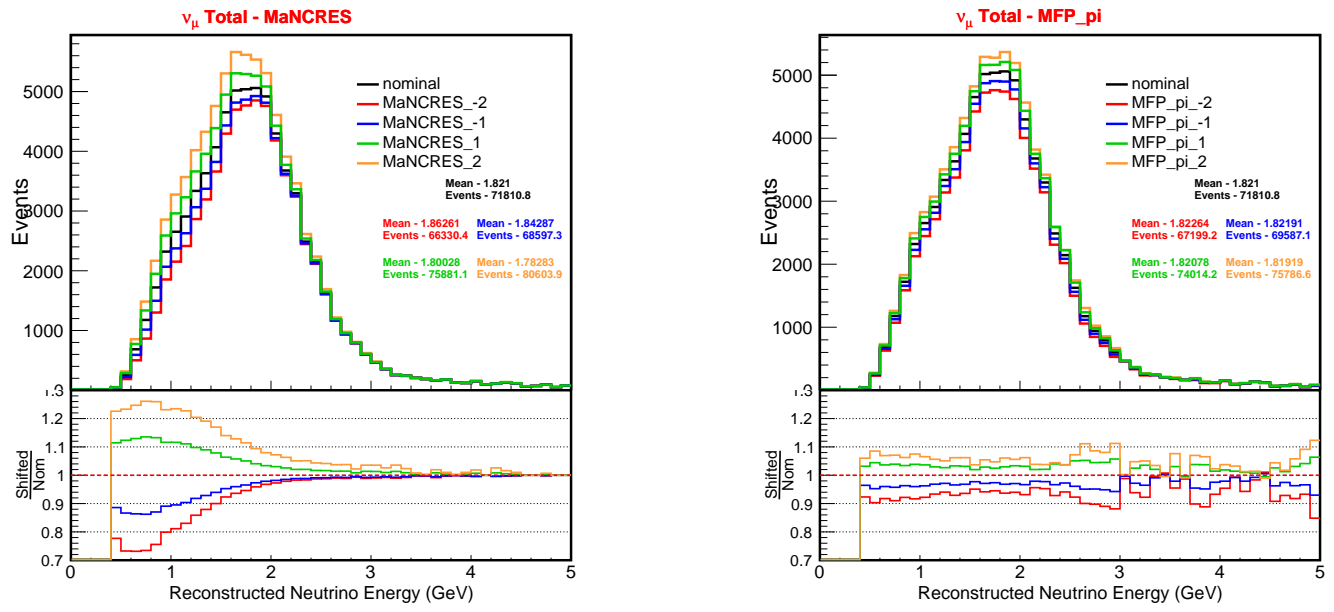

FIGURE 5.21: Shows the effect of GENIE parameter MaNCRES (left) and MFP_pi (right) on $\nu_{\mu}$ selected sample which doesn't include appeared $\nu_{e}$ 's. The effect of large genie parameters on the both $\nu_{e}$ and $\nu_{\mu}$ selected spectra is shown in Table 5.3 and 5.4. 

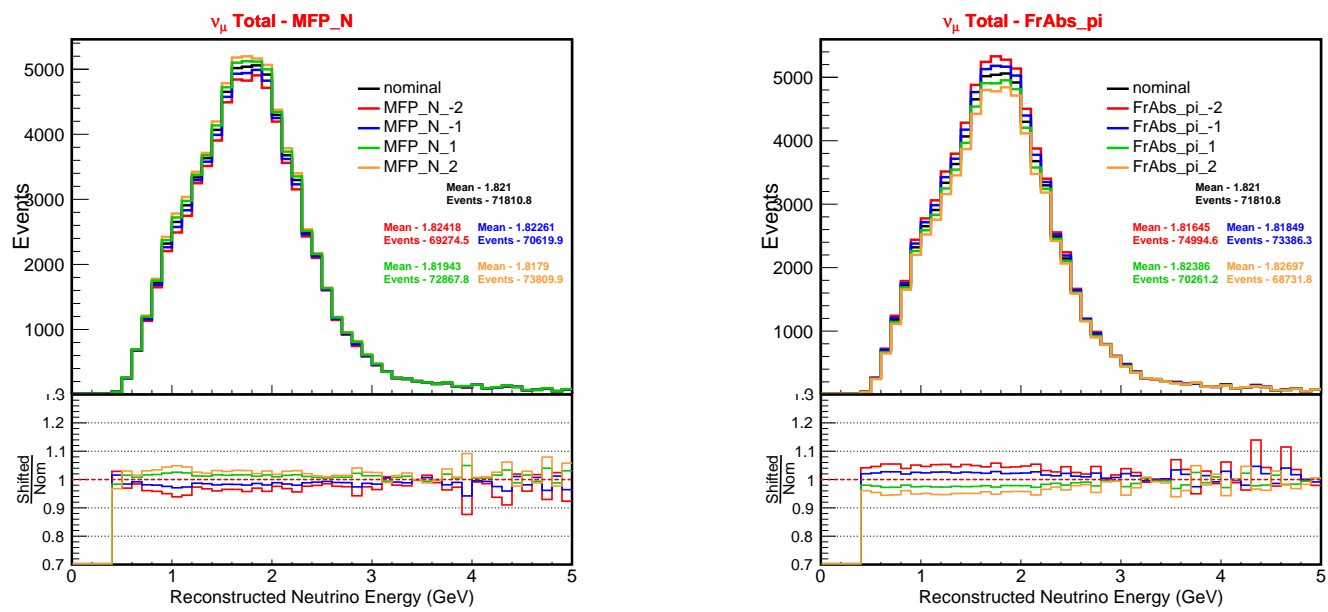

Figure 5.22: Shows the effect of GENIE parameter MFP_N (left) and FrAbs_pi (right) on $\nu_{\mu}$ selected sample which doesn't include appeared $\nu_{e}$ 's. The effect of large genie parameters on the both $\nu_{e}$ and $\nu_{\mu}$ selected spectra is shown in Table 5.3 and 5.4.
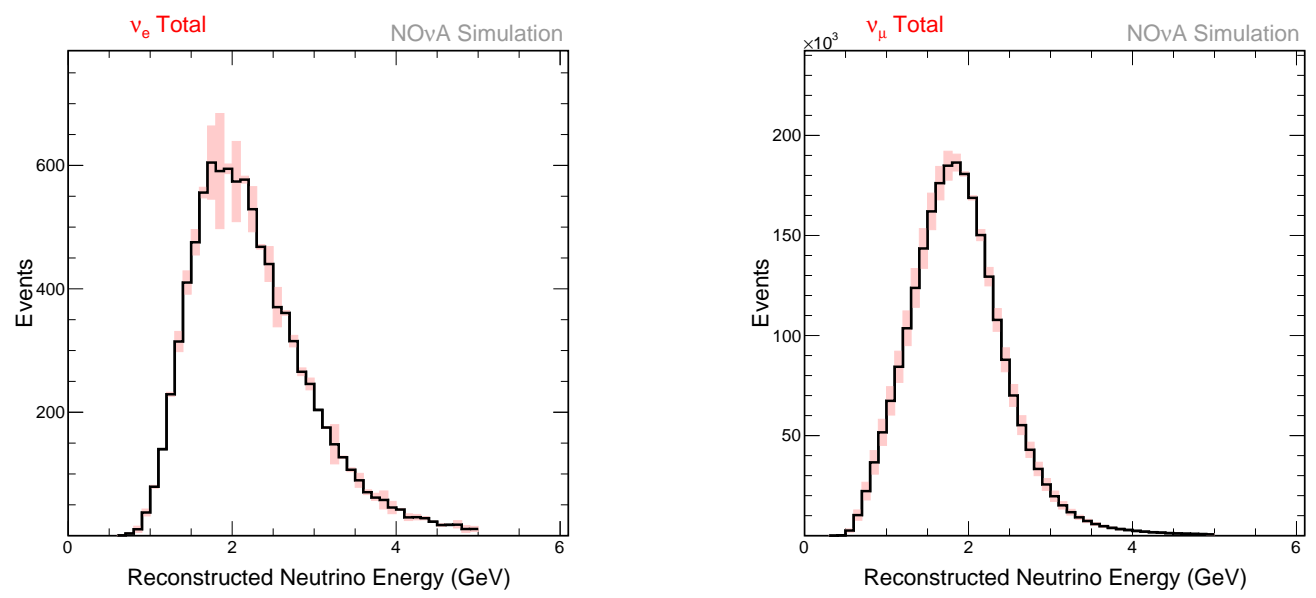

FiguRE 5.23: Effect of calibration offset shown for both $\nu_{e}$ (left) and $\nu_{\mu}$ (Right). Effect is large below $1 \mathrm{GeV}$ for $\nu_{\mu}$ 's.
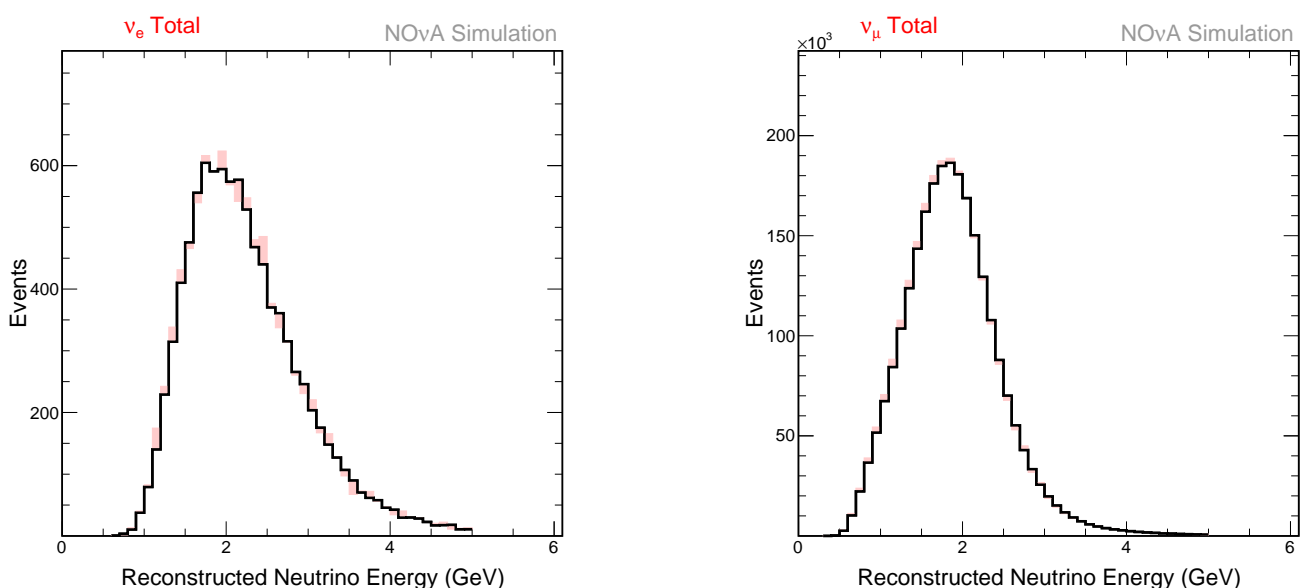

Figure 5.24: Effect of calibration shape shown for both $\nu_{e}$ (left) and $\nu_{\mu}$ (Right). Effect is large below $1 \mathrm{GeV}$ for $\nu_{\mu}$ 's. 


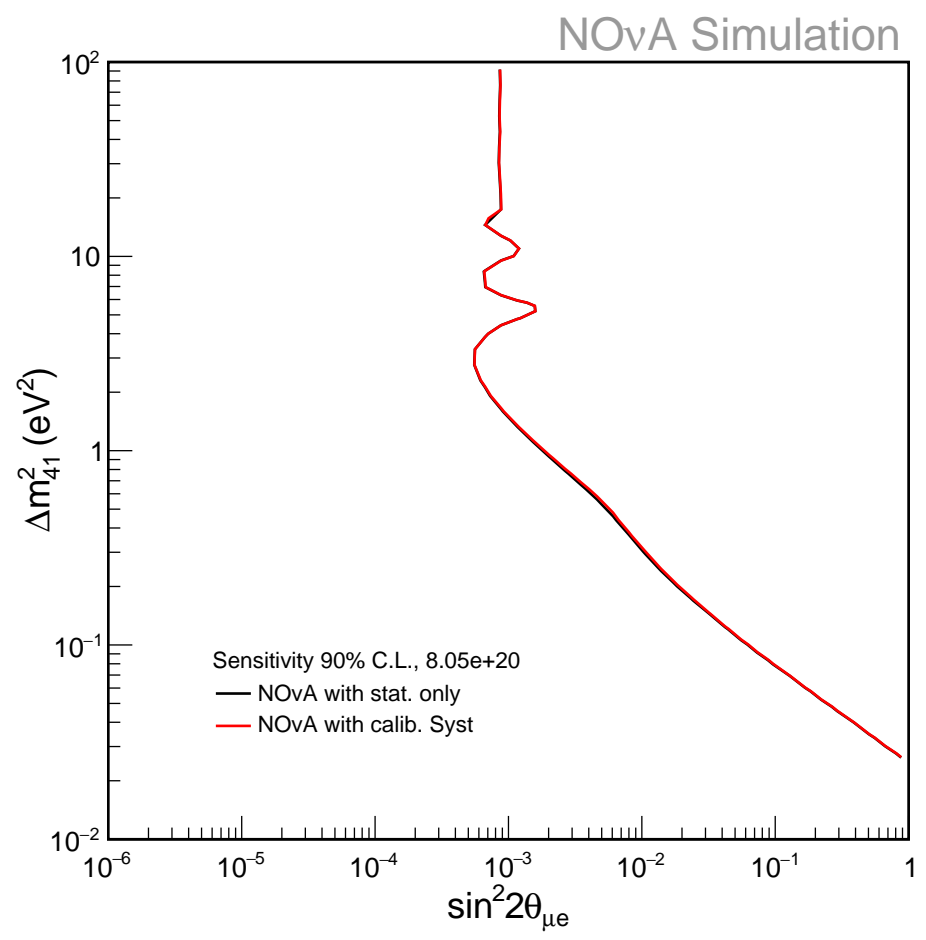

FigURE 5.25: Effect of calibration offset is shown on $\theta_{\mu e}$ limits.

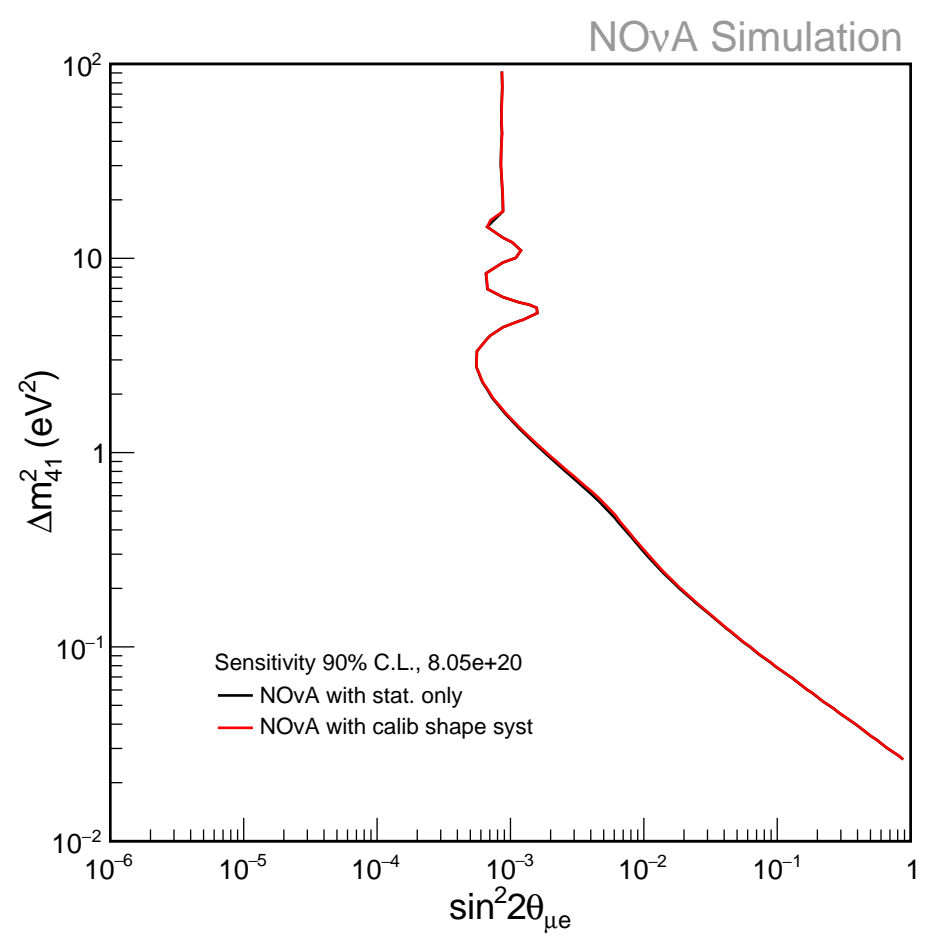

Figure 5.26: Effect of calibration shape is shown on $\theta_{\mu e}$ limits. 
The calibration shifted $\nu_{e}$ and $\nu_{\mu}$ selected components and the ratio between the shifted and nominal are shown in Fig. ??.
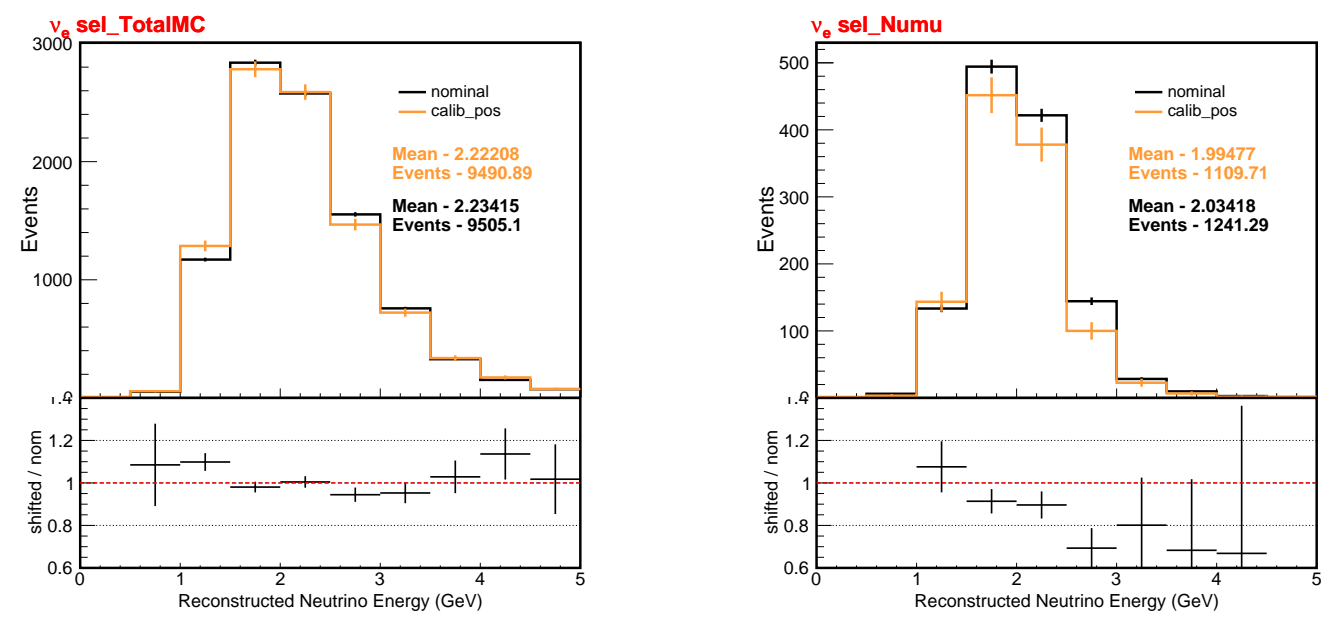

Figure 5.27: Effect of calibration + ve offset shown for $\nu_{e}$ selected TotalMC (left) and $\nu_{\mu}$ (Right).
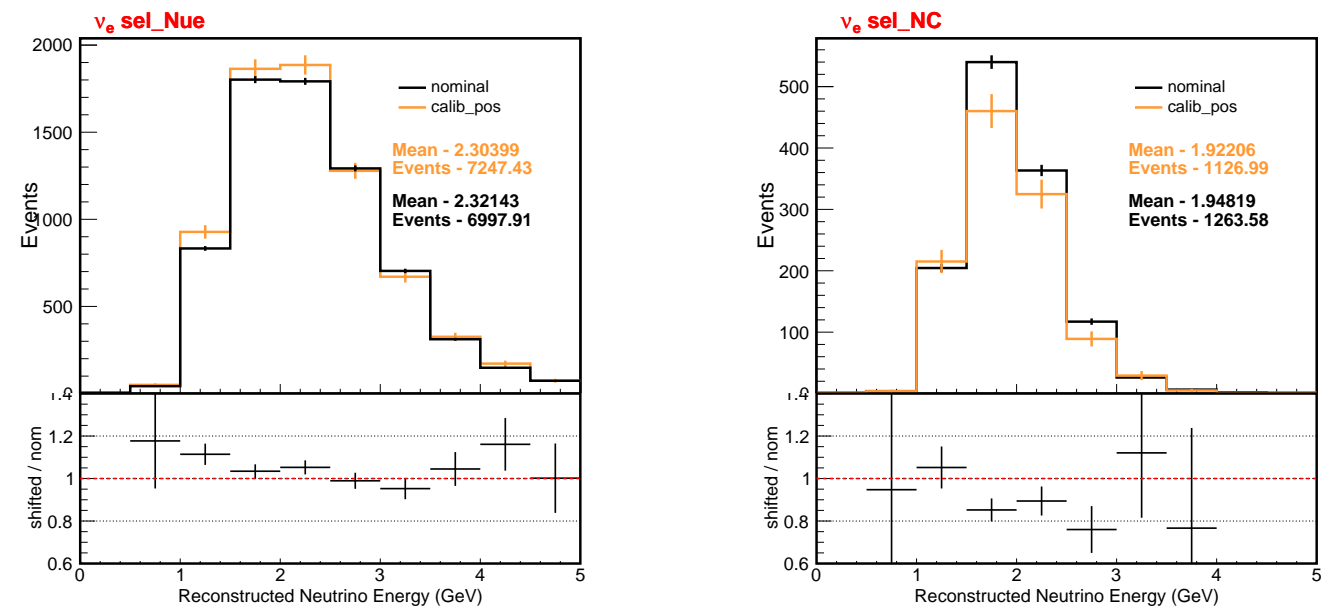

FiguRE 5.28: Effect of calibration +ve offset shown for $\nu_{e}$ selected beam $\nu_{e}$ (left) and NC (Right). 

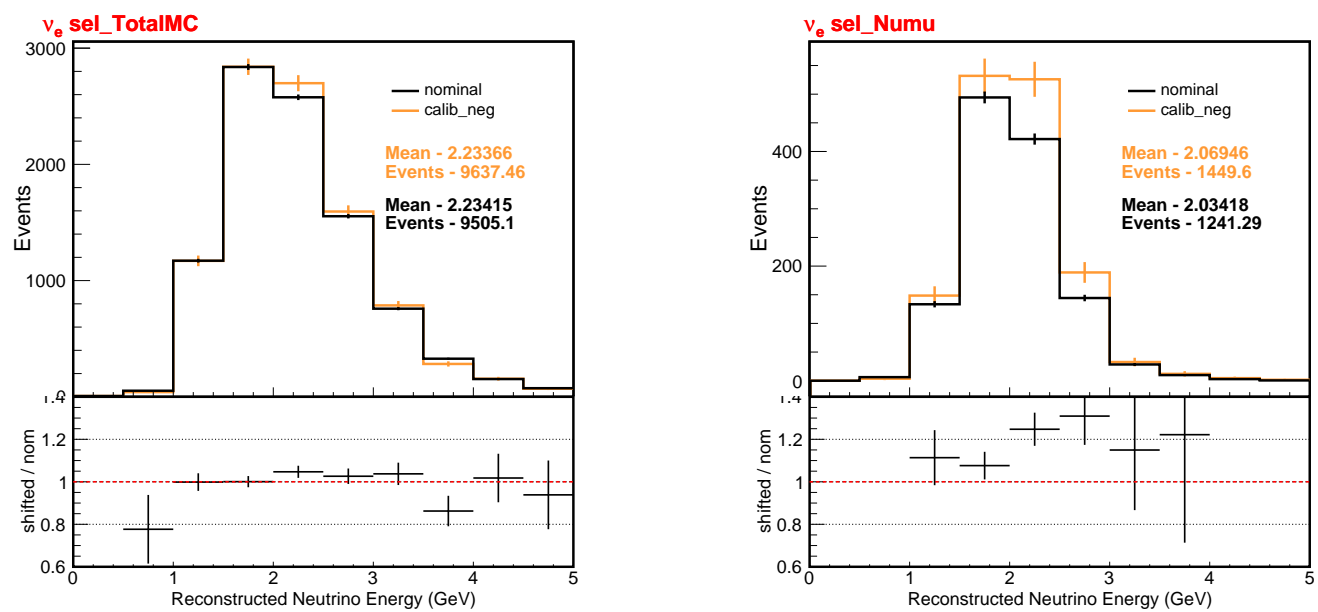

Figure 5.29: Effect of calibration -ve offset shown for $\nu_{e}$ selected TotalMC (left) and $\nu_{\mu}$ (Right).
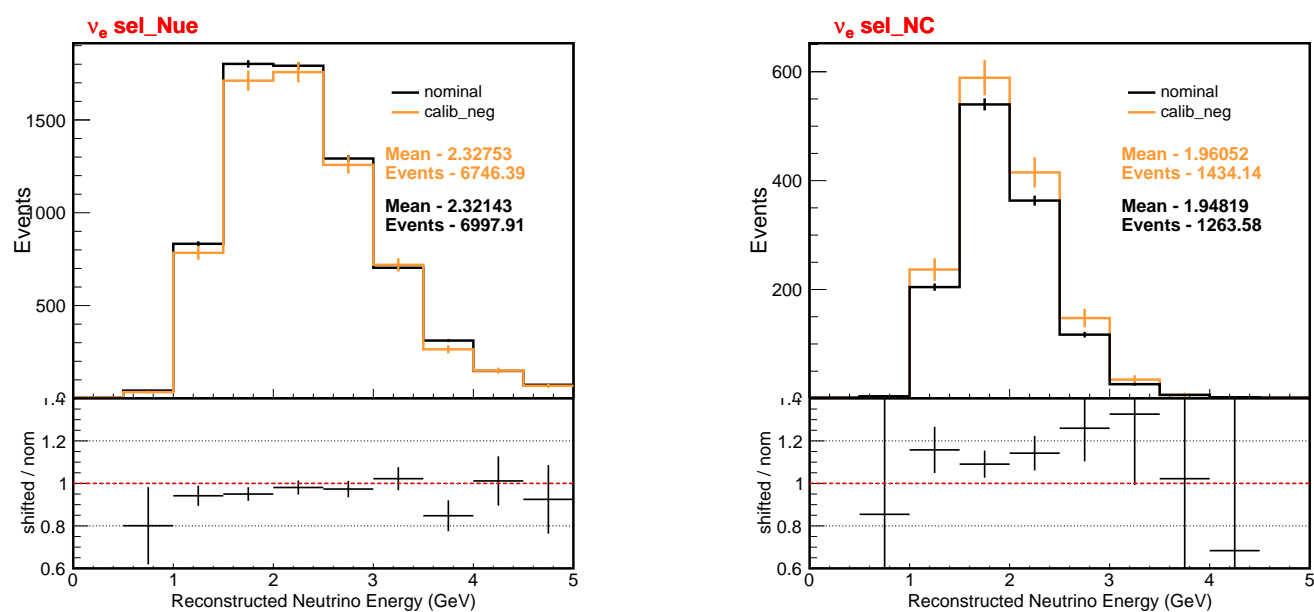

FIGURE 5.30: Effect of calibration -ve offset shown for $\nu_{e}$ selected beam $\nu_{e}$ (left) and NC (Right). 

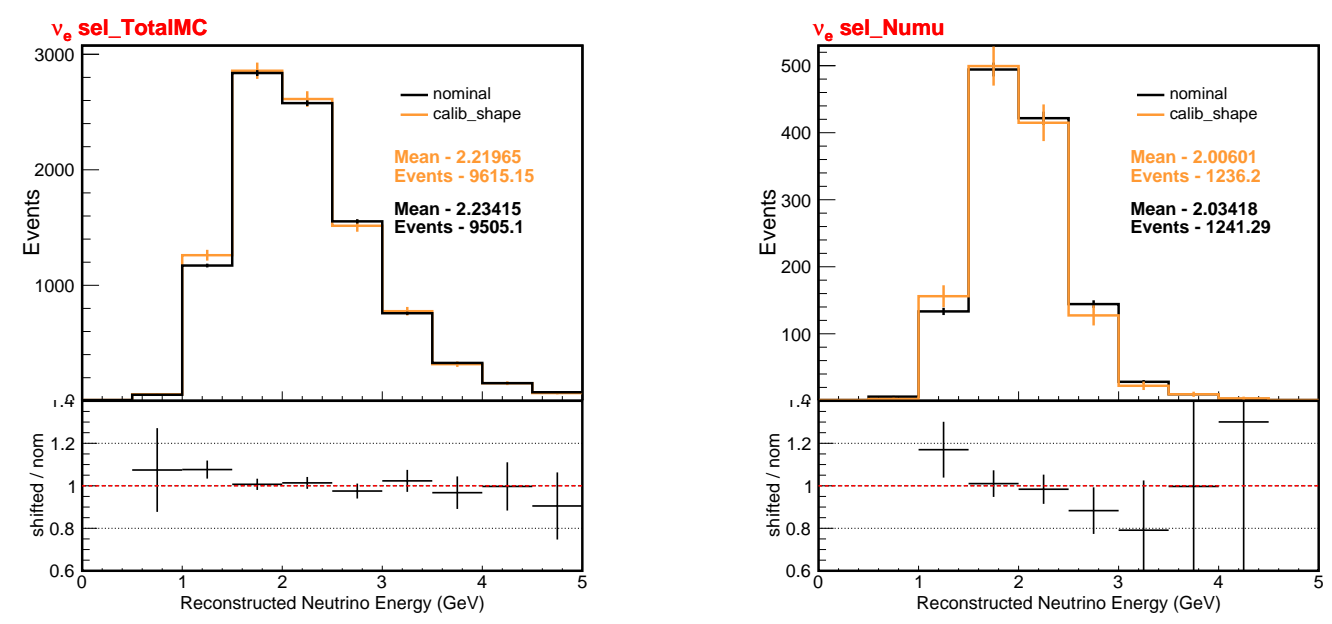

Figure 5.31: Effect of calibration shape shown for $\nu_{e}$ selected TotalMC (left) and $\nu_{\mu}$ (Right).
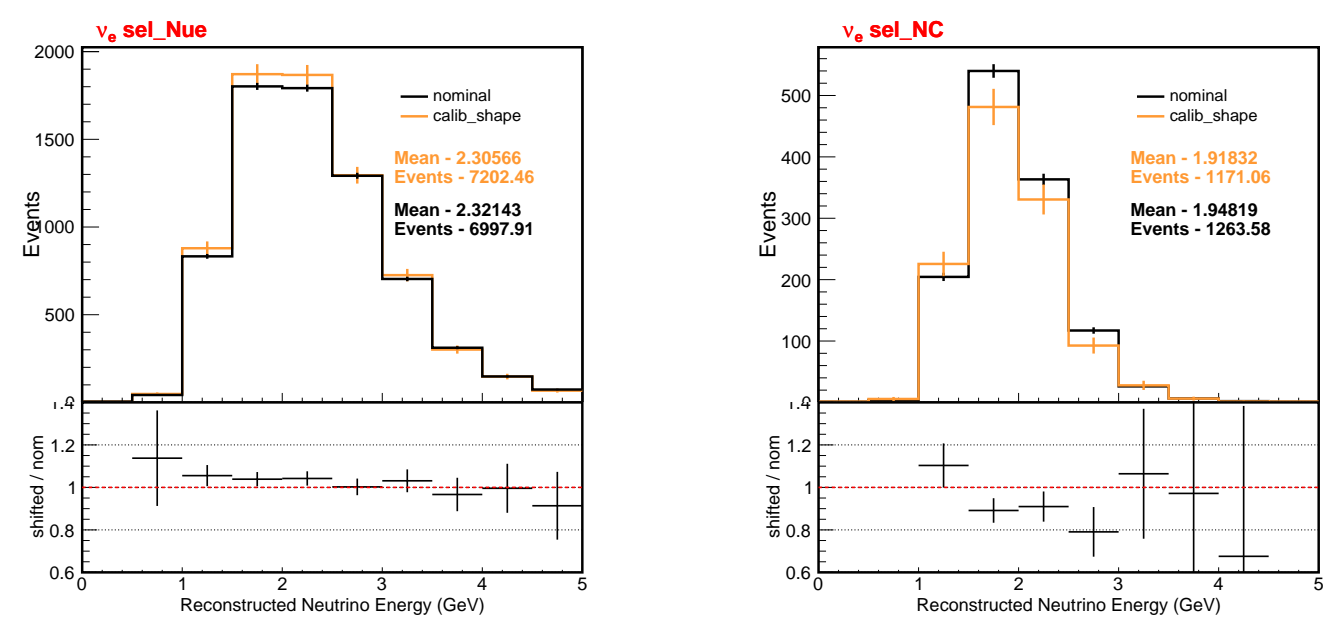

FiguRE 5.32: Effect of calibration shape shown for $\nu_{e}$ selected beam $\nu_{e}$ (left) and NC (Right). 

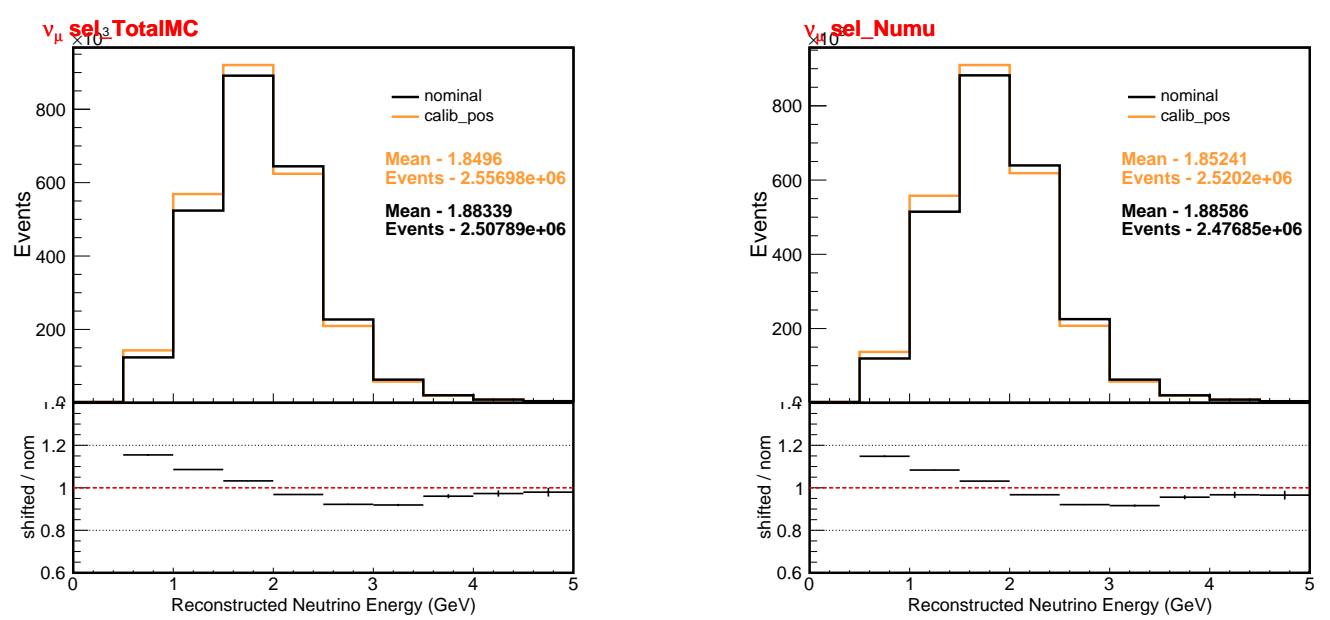

Figure 5.33: Effect of calibration +ve offset shown for $\nu_{\mu}$ selected TotalMC (left) and $\nu_{\mu}$ (Right).
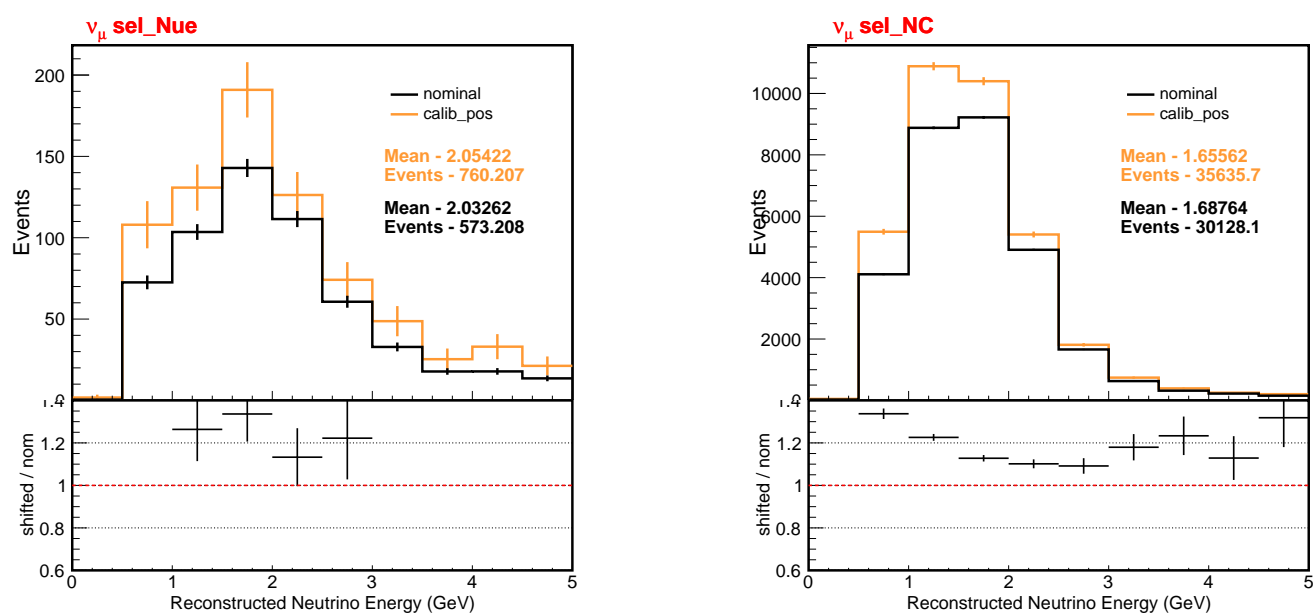

FiguRE 5.34: Effect of calibration +ve offset shown for $\nu_{\mu}$ selected $\nu_{e}$ (left) and $\nu_{\mu}$ (Right). 

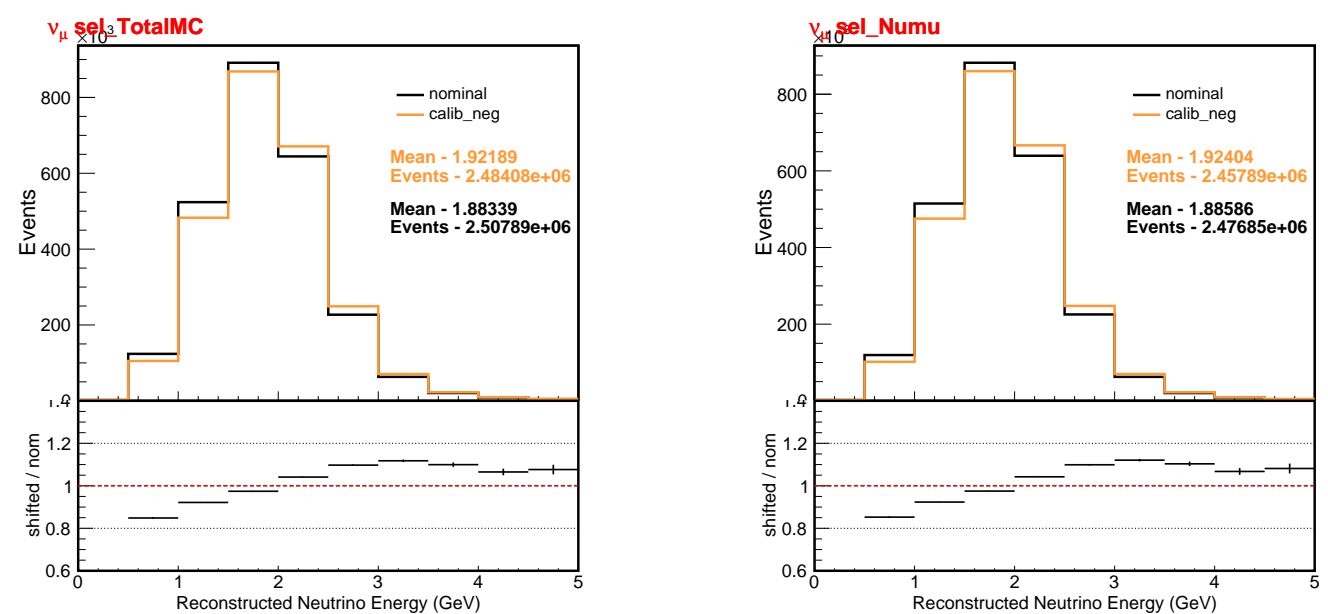

FiguRE 5.35: Effect of calibration offset shown for both $\nu_{\mu}$ selected TotalMC (left) and $\nu_{\mu}$ (Right).
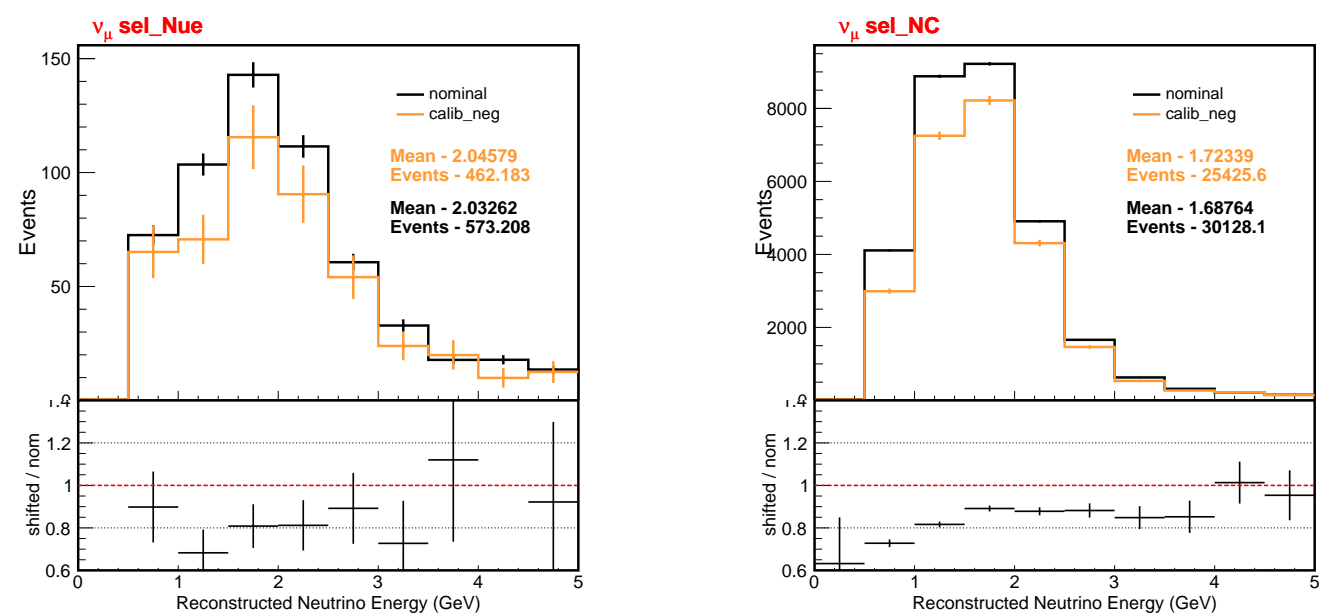

Figure 5.36: Effect of calibration -ve offset shown for $\nu_{\mu}$ selected beam $\nu_{e}$ (left) and NC (Right). 

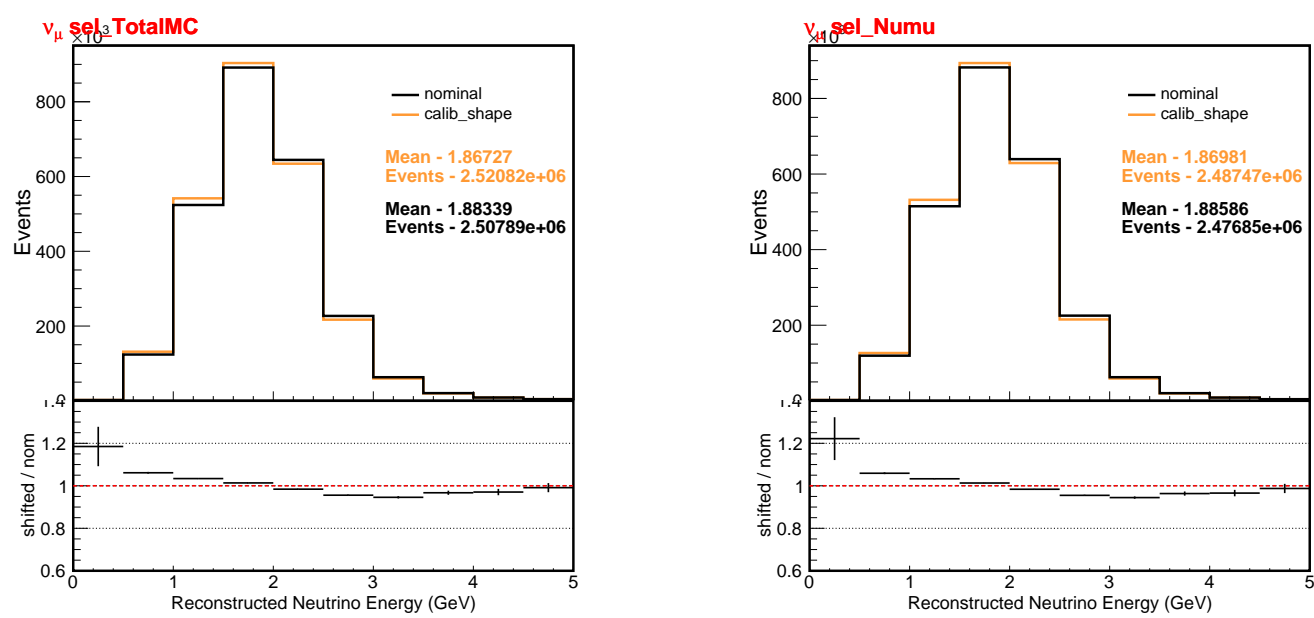

FiguRE 5.37: Effect of calibration shape shown for $\nu_{\mu}$ selected TotalMC (left) and $\nu_{\mu}$ (Right).
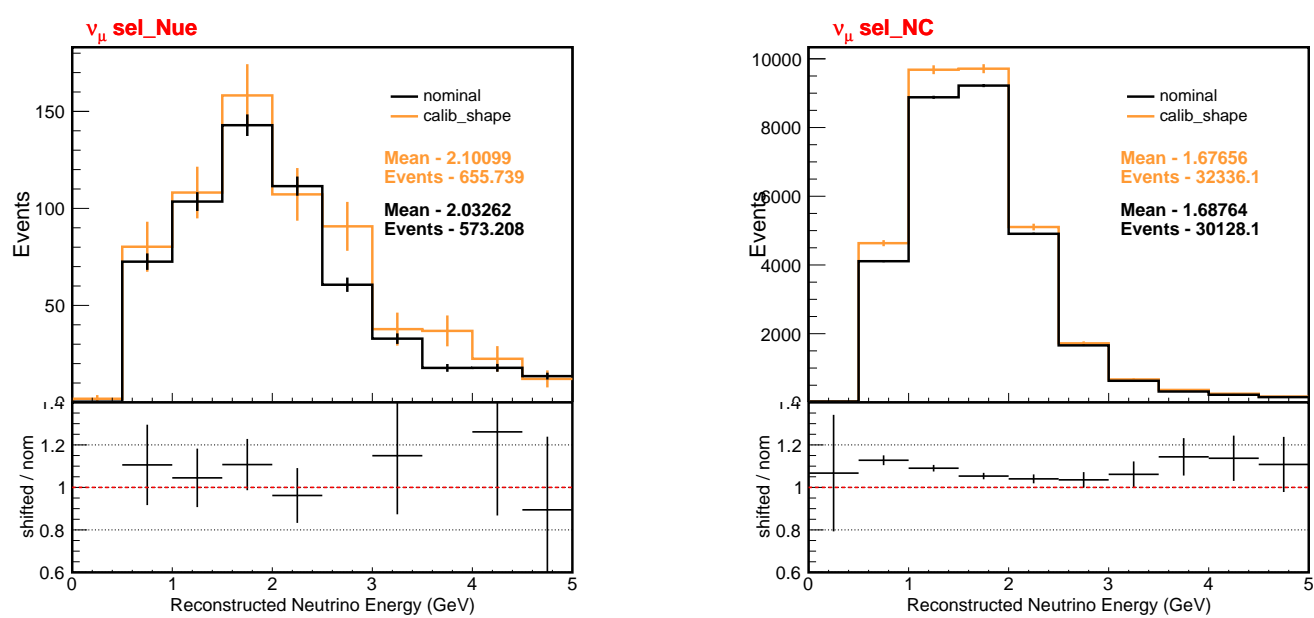

Figure 5.38: Effect of calibration offset shown for $\nu_{\mu}$ selected beam $\nu_{e}$ (left) and NC (Right).

\subsection{Rock Systematics}

Some beam neutrinos interact in the rock that surrounds the NOvA ND and consequently can then interact with the detector's fiducial volume. Rock events normally consist of a muon track with vertex outside the detector, but there are also neutrons and other particles produced. These can mimic the $\nu_{e}$ or $\nu_{\mu}$ signal. As rocks may not be well modeled in the NOvA simulation, we assign a conservative systematic uncertainty of $100 \%$ of the selected rock rate. As there are no samples without rock overlay, we determine the rock rate by applying truth containment to make sure that the true vertex resides inside the detector volume and compare the reconstructed energy distribution with and without this true vertex containment. The effect is shown in Fig. 5.39 and 
can be seen to be small for the $\nu_{e}$ selected sample. We apply the rock systematic to the $\nu_{\mu}$ sample only. This does not cover the effect due to pile-up by rock events.
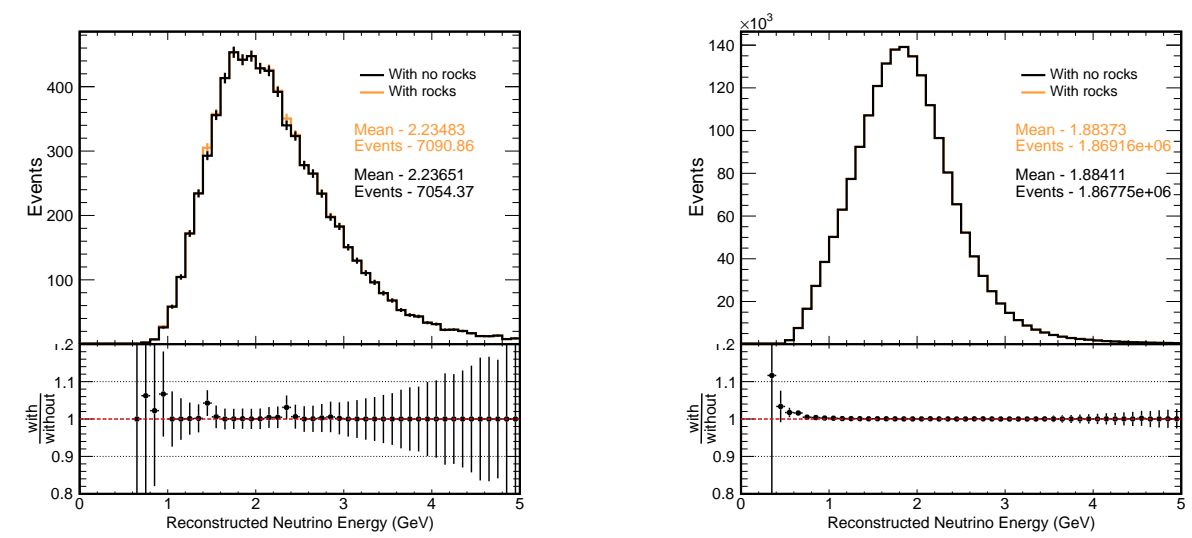

Figure 5.39: Showing the effect of rocks for both $\nu_{e}$ (left) and $\nu_{\mu}$ (right) selected sample. Effect is large below $1 \mathrm{GeV}$ for $\nu_{\mu}$ 's.

\subsection{Light Level And Cherenkov Systematics}

The uncertainties in the light level and thresholds is accounted for by considering a $10 \%$ shift in the light level and a compensating change in the absolute calibration constants. We show the effect of a shift in light levels on both $\nu_{e}$ and $\nu_{\mu}$ predictions in Fig. 5.40, and on $\theta_{\mu e}$ limits in Fig. 5.41. Also, a 2.6\% down shift is taken for the proton response in the C̆herenkov model to account for the miscalibration of hadrons, particularly protons, which assume no C̆herenkov photons emitted. The effect of the C̆herenkov systematic both the $\nu_{e}$ and $\nu_{\mu}$ predictions can be seen in Fig. 5.42 and on $\theta_{\mu e}$ sensitivity in Fig. 5.43. We observe that the effect of the light level and C̆herenkov is seemingly negligible on the final sensitivity.
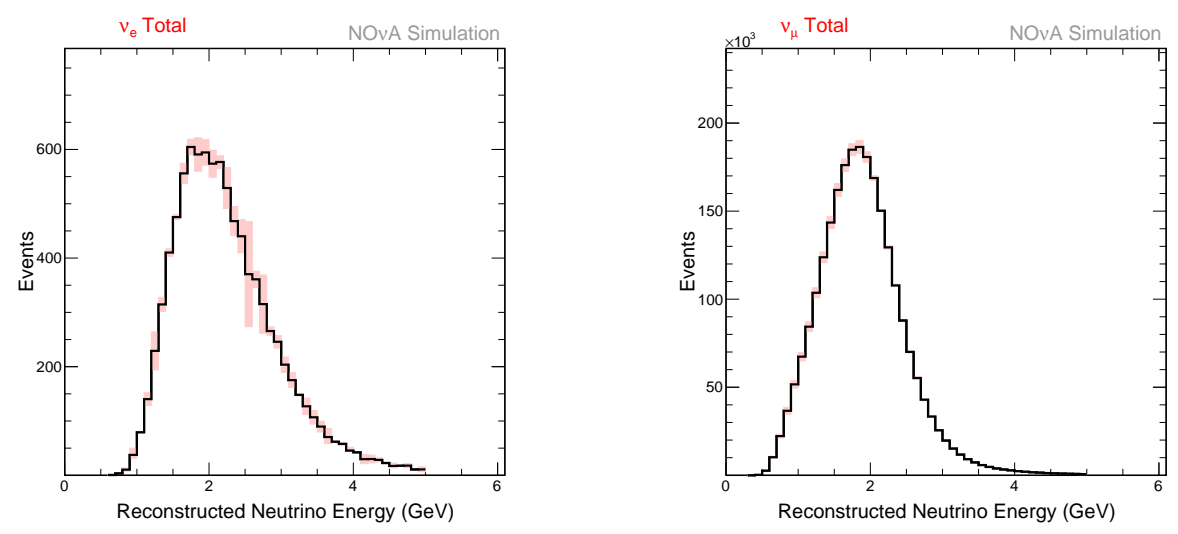

Figure 5.40: Effect of light level is shown for both $\nu_{e}$ (left) and $\nu_{\mu}$ (right). Effect is large below $1 \mathrm{GeV}$ for $\nu_{\mu}$ 's. 


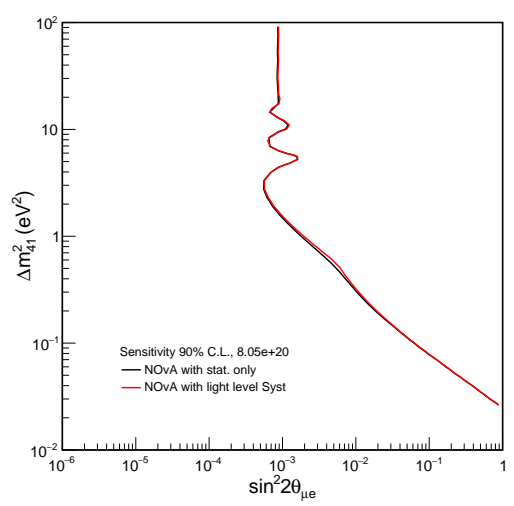

FIGURE 5.41: Effect of light level systematics on the sensitivity.
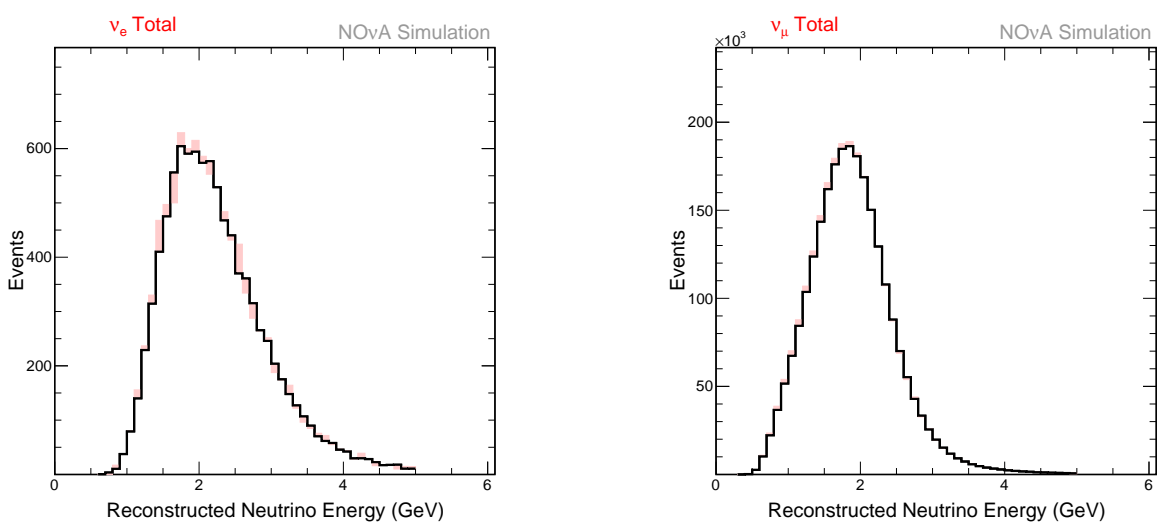

FIGURE 5.42: Effect of cherenkov is shown for both $\nu_{e}$ (left) and $\nu_{\mu}$ (right). Effect is large below $1 \mathrm{GeV}$ for $\nu_{\mu}$ 's.

\subsection{Energy Bias}

This study quantifies any potential bias in the true energy of the appeared $\nu_{e}$ as compared to the beam $\nu_{\mu}$ 's due to our energy estimation and reconstructed-to-true translation. This covers any effect where the disappearing $\nu_{\mu}$ spectra would not map to appearing $\nu_{e}$ of the same energy.

In Fig. 5.44, we show the distribution of true energy for appeared $\nu_{e}$ and beam $\nu_{\mu}$ events without any selection applied except removing rock events by ensuring a true vertex is within the full detector volume. The effect at low energies is due to expected intrinsic differences in the cross sections, as you can see in the right plot in Fig. 5.44, but otherwise they match as expected.

We quantify the effect by comparing the difference in our signal (i.e. for both the selected disappearing $\nu_{\mu}$ and selected appearing $\nu_{e}$ ). By comparing the true energy of selected 


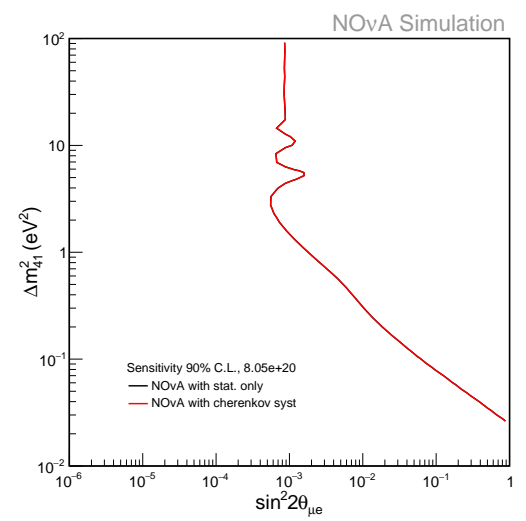

Figure 5.43: Effect of Cherenkov systematics on $\theta_{\mu e}$ limits is shown.

events and the true energy as determined by the reconstructed to true translation in the CAF files.

In the case of oscillations of active to sterile neutrinos, we need to account for the varying true baseline length, L, for the different neutrino events (unlike for the FD which can be treated at as point at a fix L). Therefore, the oscillation probabilities depend on a varying true baseline length, L, and true energy, E. So this uncertainty is actually included as true L/E and not true E. In Fig. 5.45, we plotted the ratio between true $\mathrm{L} / \mathrm{E}$ from extrap and CAF's for both $\nu_{e}$ and $\nu_{\mu}$. This leads to $<1 \%$ effect as you can see in Fig. 5.46, which specifically shows the double ratio of true over extrap of true $\mathrm{L} / \mathrm{E}$ for both $\nu_{e}$ and $\nu_{\mu}$.
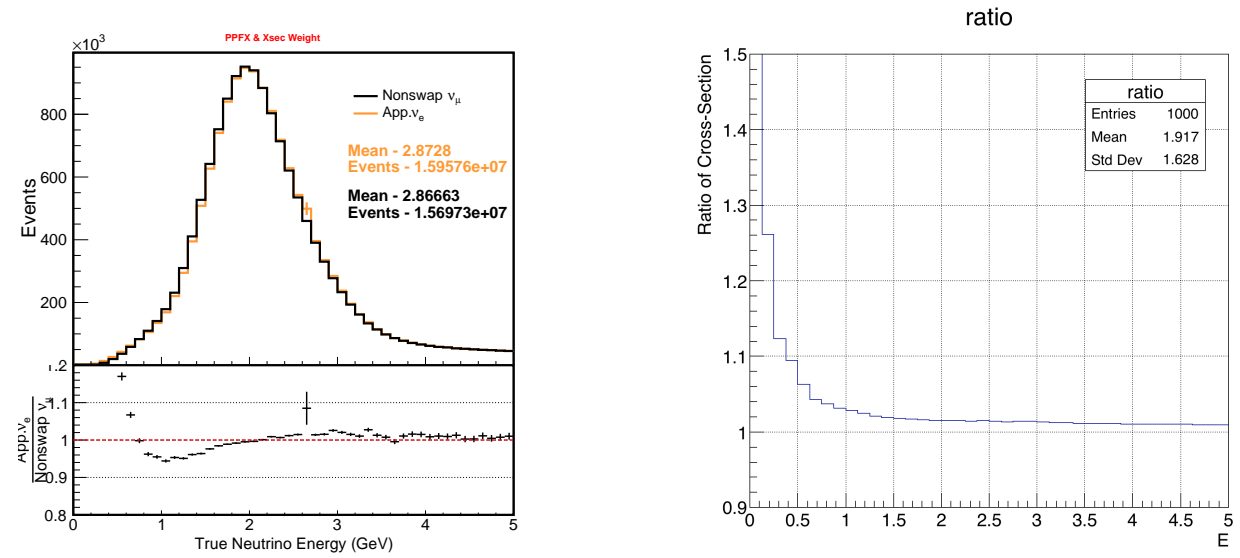

Figure 5.44: The distribution of true energy for flux swap $\nu_{e}$ 's and beam $\nu_{\mu}$ 's with no selection applied (left). We also applied the PPFX and cross-section weights. Right plot shows the ratio of $\nu_{e}$ and $\nu_{\mu}$ cross-sections. 

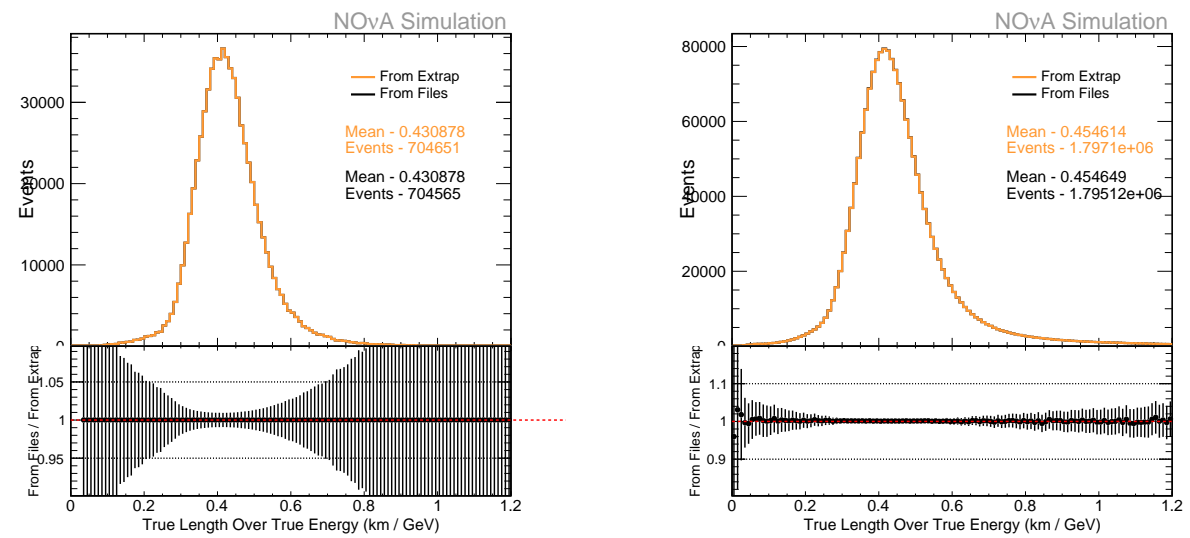

Figure 5.45: The distribution of true length over true energy for the $\nu_{e}$ selected appeared $\nu_{e}$ 's and $\nu_{\mu}$ selected beam $\nu_{\mu}$ 's. The orange histogram is a projection onto $\mathrm{L} / \mathrm{E}$ axis from the $2 \mathrm{D}$ spectrum in extrapolation object.

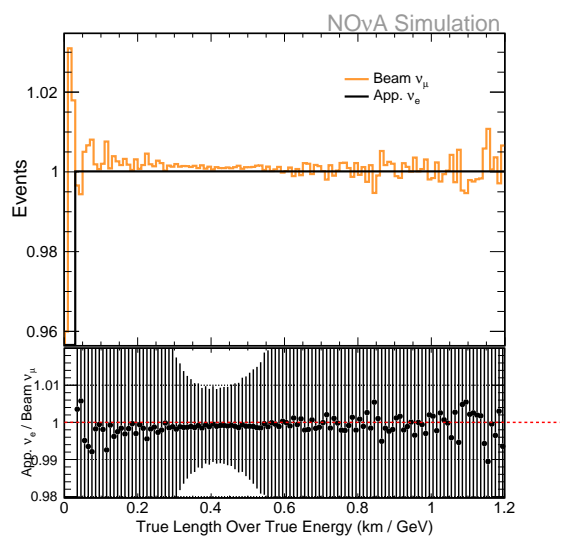

Figure 5.46: The double ratio of true / extrap as true L/E for both appeared $\nu_{e}$ and $\nu_{\mu}$ 's. The double ratio is flat across true $\mathrm{L} / \mathrm{E}$. 


\subsection{Muon Energy Scale}

This is only included for muons in the $\nu_{\mu}$ sample. It is estimated from Ref. [121]. An absolute error of $0.96 \%$ and $0.76 \%$ is accounted for the ND active volume and muon catcher, respectively.

\subsection{Normalization Systematics}

There are multiple systematics which we include that only have an effect on the overall normalization. They are:

\subsubsection{POT Accounting}

As observed in the second analysis and documented in [122]. This systematic accounts for the drift in the ratio of the calibrated response of TRTGTD and TR101D. It is kept the same size as for the second analysis: a $0.5 \%$ overall normalization effect for the near detector.

\subsubsection{Detector Mass}

Intensive work is done to get the correct mass accounting for both the detectors which are documented in Ref. [121]. From this note, we consider a $0.27 \%$ overall normalization effect for ND alone for the mass accounting. 


\subsubsection{Detector Acceptance}

An acceptance study was performed to look at the event rates in different quadrants of the detector to account for any differences in the $\nu_{\mu}$ and $\nu_{e}$ acceptances across the ND. For this study, we looked at the relative Data/MC differences in selected events that have a reconstructed vertex in different quadrants and get the relative difference of Data/MC difference between these quadrants. We do not include shape information as this analysis keeps the ND Data blind, but they are expected to be small.

We divide the front face of the detector into four parts (quadrants) that extend the length of the Z-axis, as itemized below and depicted in Fig. 5.47. Since we blind the analysis from any energy-related information, we look at the distribution of reconstructed variables like the number of vertices, vertex position, number of tracks, etc., after applying the $\nu_{e}$ and $\nu_{\mu}$ full selection within each quadrant for both MC and Data. We then get the relative difference of Data and $\mathrm{MC}$ between the four quadrants as the final systematic, which is obtained as $2 \%$ for both $\nu_{e}$ and $\nu_{\mu}$. We consider this as an overall uncorrelated normalization effect. One can find further details on this study in Ref. [123].

- $\mathrm{X}:-100<\operatorname{vertX}<160 ; \quad \mathrm{X} 1:-100<\operatorname{vertX}<0 ; \quad \mathrm{X} 2: 0<\operatorname{vertX}<160$;

- $\mathrm{Y}:-160<\operatorname{vert} \mathrm{Y}<100 ; \quad \mathrm{Y} 1:-160<\operatorname{vert} \mathrm{Y}<0 ; \quad \mathrm{Y} 2: 0<\operatorname{vert} \mathrm{Y}<100$;

- Z: $150<\operatorname{vertZ<900;~}$

- Fiducial volumes defined as XIY1Z, X1Y2Z, X2Y1Z, X2Y2Z.

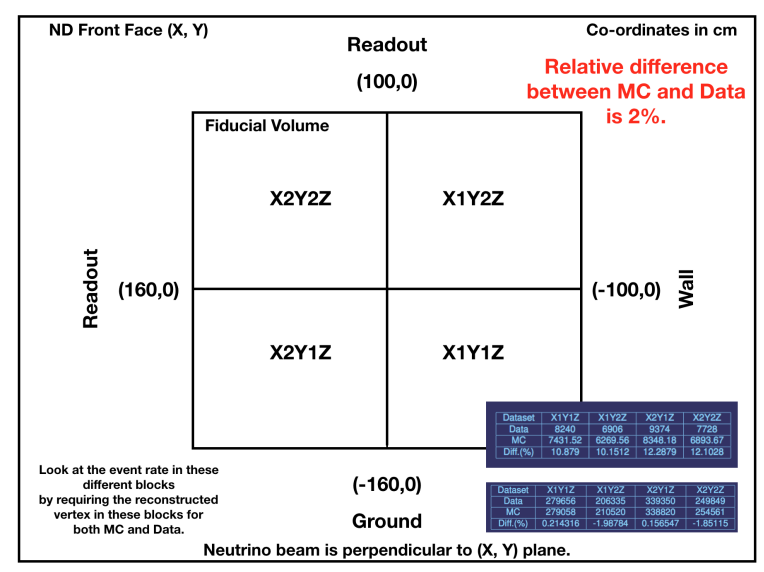

Figure 5.47: Showing the method followed to get an estimate of the acceptance effect using the $\nu_{e}$ and $\nu_{\mu}$ selected sample. 


\subsection{Selection Efficiency of overlaid swapped $\nu_{e}$}

We use a sample of overlaid single $\nu_{e}$ (fluxswap) interactions as the appeared $\nu_{e}$ signal in the appearance analysis. These swapped $\nu_{e}$ should have the same true energy as $\nu_{\mu}$ 's except with a small difference due to differing cross-sections. As they are overlaid, we have one extra interaction per event which results in a higher POT/spill. We test if this sample observes any efficiency differences due to these overlaid events. We have to have the same selection efficiency for both beam $\nu_{e}$ 's and swapped $\nu_{e}$ 's. We define the efficiency as the ratio between events the pass the full $\nu_{e}$ selection and events that pass the $\nu_{e}$ preselection (data quality, fiducial, containment, and the front planes selection). In Fig. 5.48, we show the efficiency in true energy and the ratio between the efficiency of beam $\nu_{e}$ 's and appeared $\nu_{e}$ 's. The ratio is almost flat for energies below $3 \mathrm{GeV}$ with some fluctuations for energies above $3 \mathrm{GeV}$.

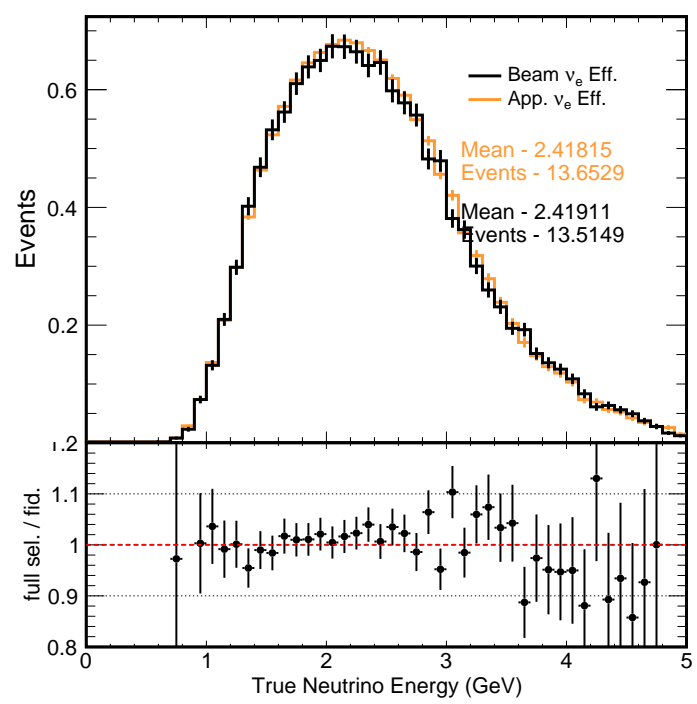

FiguRE 5.48: Show the ratio of efficiencies for both beam and appeared $\nu_{e}$ 's in true energy.

\subsection{Negligible systematic effects}

The following are effects that we studied and subsequently determined to be negligible, thus are not included in the ensemble of systematic parameters included in the fits. 


\subsubsection{Periodic Calibration Triggers: Detector Noise Mismodeling}

To study the effect of mis-modeled detector noise on the reconstruction of ND events we generated a sample where events from the periodic calibration trigger (cosmic trigger) are used as noise. This is the same way the FD cosmic-overlay files are made, where we make a new sample by overlaying noise-removed MC simulation with cosmic trigger data. We made the sample in the second analysis style, prod_caf_R16-03-03mixedcalib.a_nd_genie_nonswap_genierw_fhc_nova_v08_full_v1_cosmic-overlay-removenoise (11897 files).

As the effect obtained in the second analysis was so small, shown in Fig. 5.49, we have not remade these files for the third analysis. The third analysis uses to run, and position matched thresholds, so this effect is expected to be even smaller.

We estimate the effect of this noise by taking the ratio between the reconstructed energy distribution of $\nu_{e}\left(\nu_{\mu}\right)$ selected sample with noise and the reconstructed energy distribution of $\nu_{e}\left(\nu_{\mu}\right)$ selected sample without noise. The figure.5.49 shows the effect of cosmic noise on $\nu_{e}$ and $\nu_{\mu}$ energy distributions..
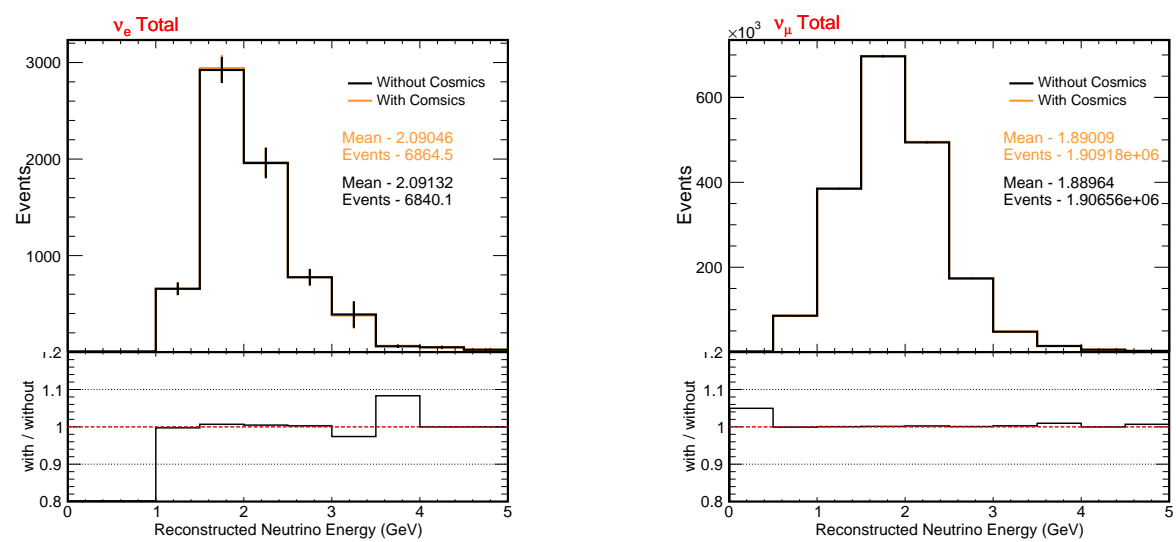

FiguRE 5.49: Showing the distribution of reconstructed energy after the $\nu_{e}$ (left) and $\nu_{\mu}$ (right) selection applied with and without cosmic noise. We use CVN selector for the selection of both $\nu_{e}$ and $\nu_{\mu}$. 


\subsubsection{Intensity Effect}

For the third analysis, Monte Carlo spills are simulated with the POT matched to NuMI spill POT in data. The distribution of spill POT for data and MC is shown in Fig. 5.50. It is expected that any intensity effect will be smaller than that of the second analysis, due to our improved modeling. The final effect of varying beam intensity explained in detail in Ref. [124] and Ref. [125], can be measured as

$$
\text { Intensity effect }=\frac{\text { Data }_{\mathrm{Avg}}-\mathrm{MC}_{\mathrm{Avg}}}{\text { Data }_{\mathrm{Avg}}}
$$

This effect is based on the slope of the distribution of the average number of slices per spill versus the spill POT, shown for both data and MC in Fig. 5.51. It is estimated that the average number of slices in data is 6.288 and in $\mathrm{MC}$ is 6.287 , and the final effect is estimated as a $0.024 \%$ effect, which is negligible compared to second analysis where we didn't have intensity matching.

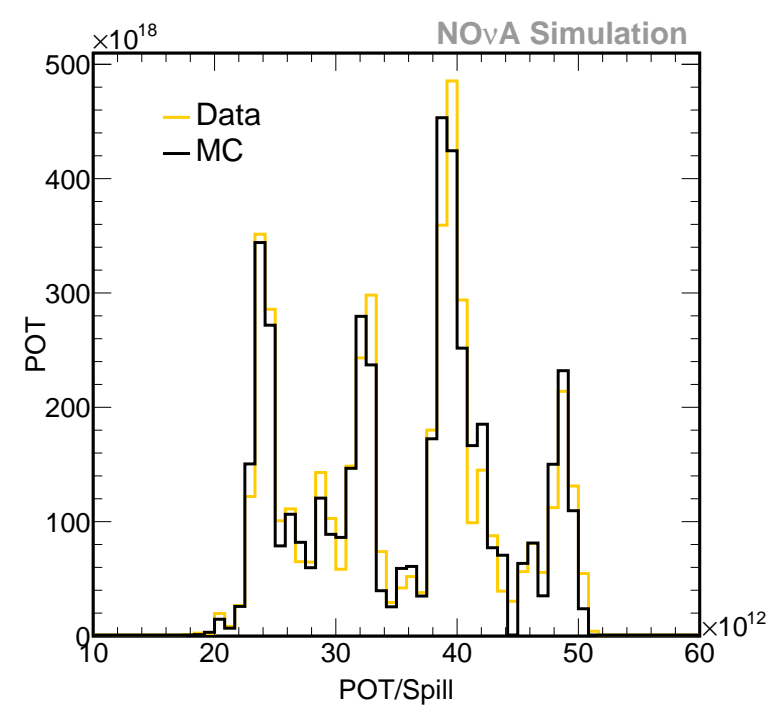

Figure 5.50: Shows the distribution of spill POT for both data and MC. 

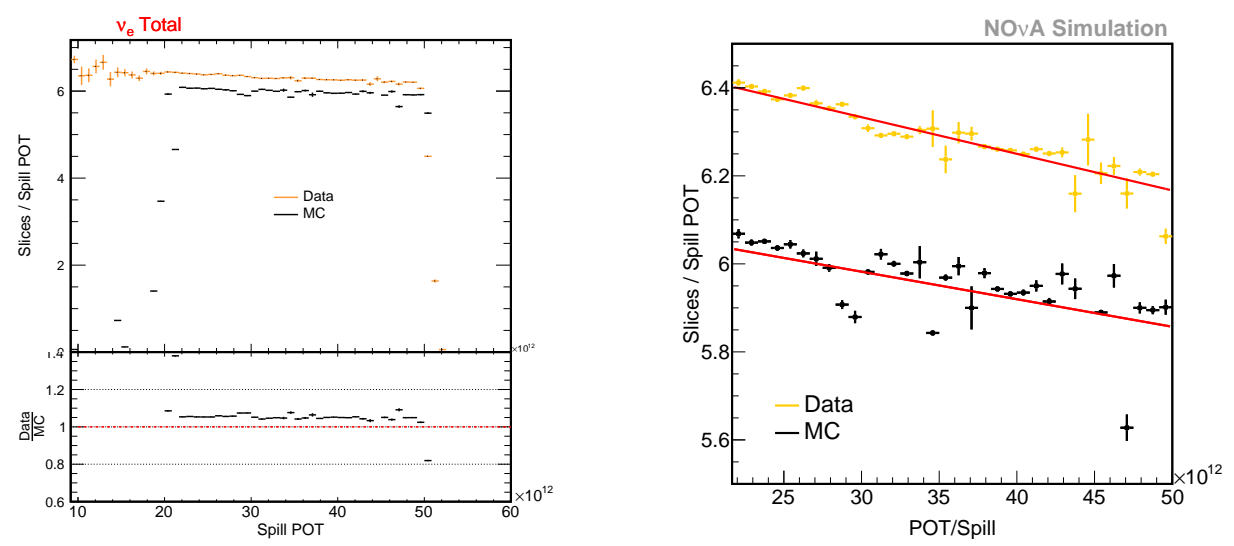

Figure 5.51: Shows the number of slices per spill POT as a function of spill POT with no selection applied (left) and CVNe $>0.95$ applied to select the $\nu_{e}$ 's. 



\section{Chapter 6}

\section{Analysis}

The anomalous results from the Liquid Scintillator Neutrino Detector (LSND) [76] and Mini Booster Neutrino Experiment (MiniBooNE) [77] experiments can be interpreted as an indication of the existence of sterile neutrinos. The NOvA Near Detector is placed at a $L / E$ centered at $\sim 0.5 \mathrm{~km} / \mathrm{GeV}$, consistent with the L/E range probed by LSND and MiniBooNE, providing an opportunity to probe for the signature of active to sterile neutrino oscillations. This analysis is based on the search for simultaneous $\nu_{e}$ appearance and $\nu_{\mu}$ disappearance in the NuMI neutrino beam at the Near Detector. We select both a $\nu_{e}$ and a $\nu_{\mu}$ sample using respective selections at the Near Detector and perform a joint fit to $\nu_{e}$ appearance and $\nu_{\mu}$ disappearance. We use the NuMI neutrino dataset corresponding to $8.05 \times 10^{20}$ protons-on-target (POT) and the Production 3 Monte Carlo samples.

The LSND experiment observed a $3.8 \sigma$ excess of $\bar{\nu}_{e}$ in a $\bar{\nu}_{\mu}$ beam. Interpreting the observed excess of $\bar{\nu}_{e}$ events as due to oscillations gives to a $\bar{\nu}_{e}$ appearance probability as $(0.264 \pm 0.067 \pm 0.045) \%$. The corresponding best-fit value is $\left(\sin ^{2} 2 \theta_{\mu e}, \Delta \mathrm{m}^{2}\right)=$ $\left(0.003,1.2 \mathrm{eV}^{2}\right)$. The current global best fit is $\left(\sin ^{2} 2 \theta_{\mu e}, \Delta \mathrm{m}^{2}\right)=\left(0.00048,1.7 \mathrm{eV}^{2}\right)$ using the calculations from $[87,88]$.

To demonstrate the effect expected at the Near Detector, Figure 6.1 shows the oscillation probabilities for $\nu_{e}$ appearance and $\nu_{\mu}$ disappearance as a function of $L / E$. Figures 6.2, 6.4, and 6.5 show the distributions of the $L / E$ ratio, $L$, and $E$ respectively for $\nu_{\mu}$ and beam $\nu_{e}$ at the Near Detector. In Fig. 6.3, we show the expected appeared $\nu_{e}$ 's as a function of true $L / E$ before and after applying the $\nu_{e}$ selection at the Near Detector. 

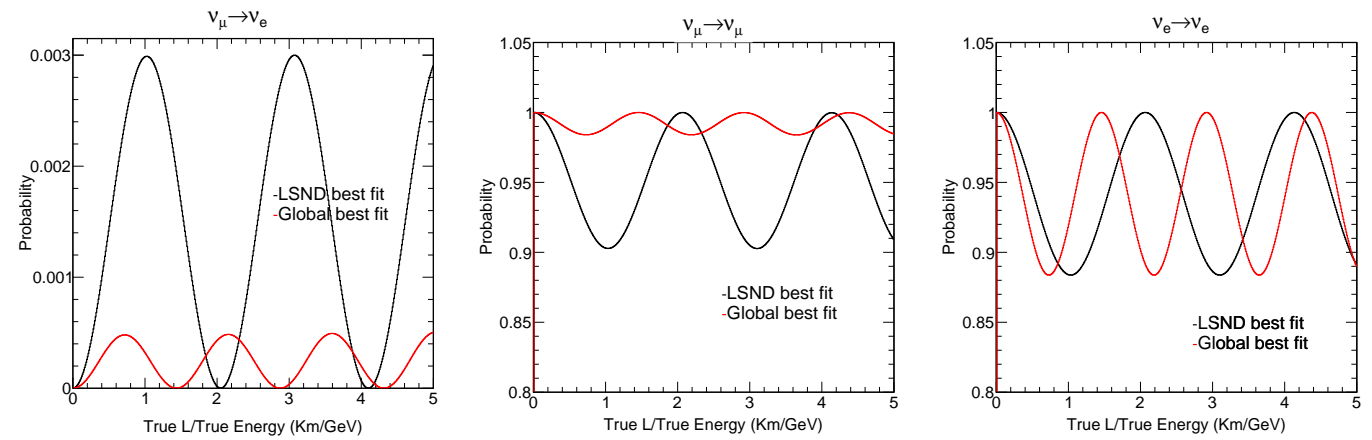

Figure 6.1: The $3+1$ model probabilities for both $\nu_{e}$ appearance (left), $\nu_{\mu}$ disappearance (middle) and $\nu_{e}$ disappearance (right) at the LSND best fit point $\left(\sin ^{2} 2 \theta, \Delta \mathrm{m}^{2}\right)=$ $\left(0.003,1.2 \mathrm{eV}^{2}\right)$ and the global best-fit $\left(\sin ^{2} 2 \theta, \Delta \mathrm{m}^{2}\right)=\left(0.00048,1.7 \mathrm{eV}^{2}\right)[87,88]$. The probability at the LSND best-fit is shown in black and the probability for the global best-fit is shown in red. To use the LSND limits which are provided in $\theta_{\mu e}$, we set $\theta_{14}$ to 10 degrees driven by the Bugey limits on this angle.
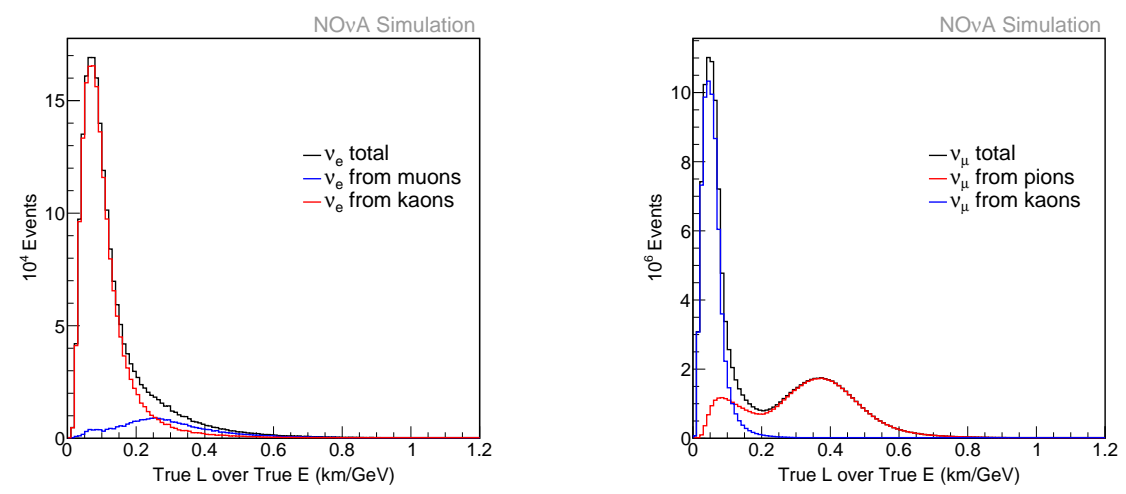

Figure 6.2: True L/E for all beam $\nu_{e}$ 's (left) and $\nu_{\mu}^{\prime} s$ (right) at the NOvA near detector.

\subsection{SBL Oscillation Analysis Overview}

The signature of sterile neutrinos at NOvA would be appearing $\nu_{e}$ 's from $\nu_{\mu} \rightarrow \nu_{e}$ oscillations, with a flux profile consistent with the Near Detector $\nu_{\mu}$ 's and disappearing $\nu_{\mu}$ 's. In this analysis we fit for both $\nu_{e}$ appearance and $\nu_{\mu}$ disappearance, due to active to sterile mixing, simultaneously at the Near Detector. We select two samples, one of $\nu_{e}$ and one $\nu_{\mu}$, with selections similar to those used by the 2016 (second analysis) oscillation analyses.

In the FD oscillation analysis, we can approximate all neutrinos starting at a point source with a fixed $L$. But for an ND oscillation analysis, however, the variation in the distance the mesons travel before decaying; hence the distance the neutrinos travel before reaching the detector (Figure 6.4), must be accounted for. The oscillation probabilities will depend on this distance $L$, as well as on the neutrino energy $E$ (Figure 6.5). We have implemented length-dependent oscillations in the current analysis framework (see 

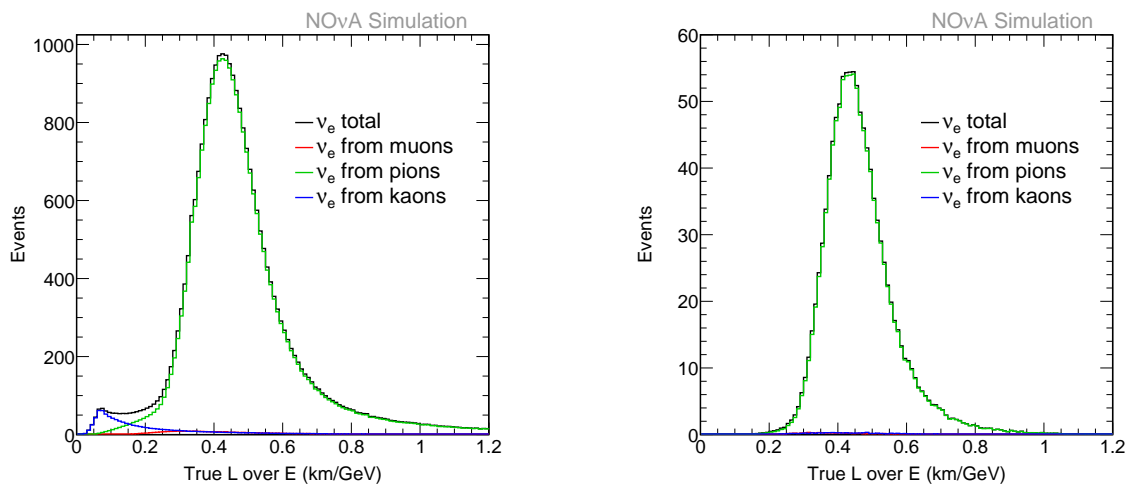

FIGURE 6.3: The number of appeared $\nu_{e}$ 's at LSND best-fit point as a function of true $\mathrm{L} / \mathrm{E}$ with no selection (left) and with $\nu_{e}$ selection applied (right) at the NOvA Near Detector.
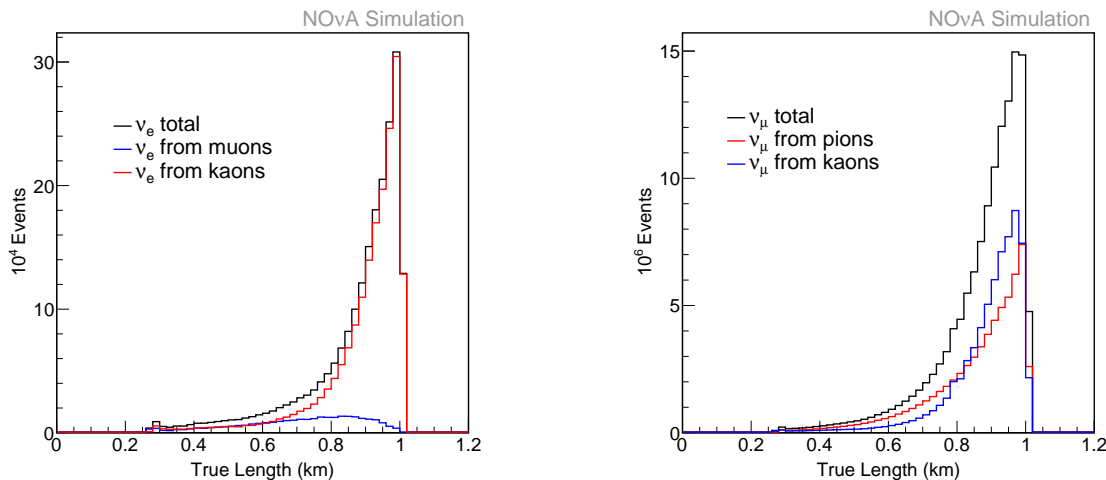

Figure 6.4: True L for all beam $\nu_{e}$ 's (left) and $\nu_{\mu}$ 's (right) at the NOvA near detector.
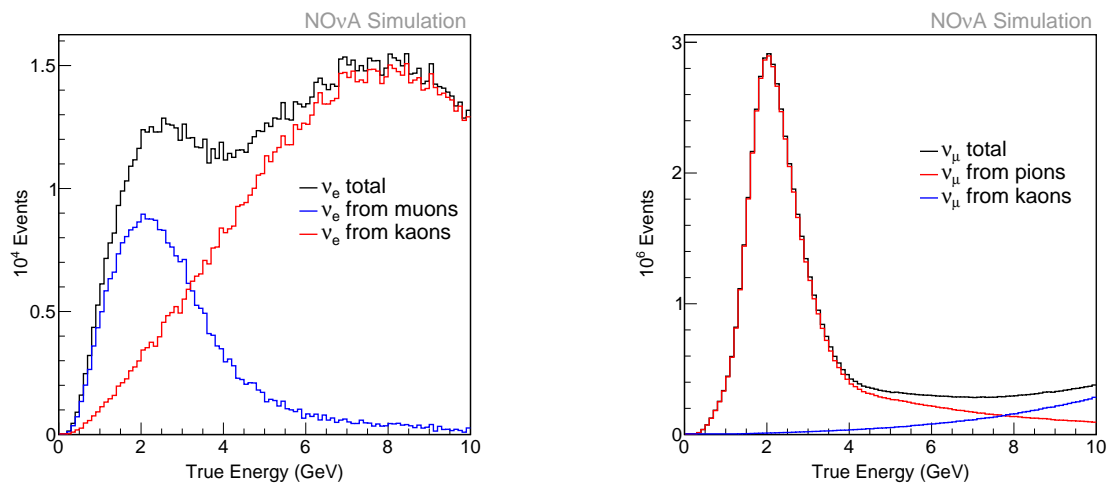

Figure 6.5: True E for all beam $\nu_{e}$ 's (left) and $\nu_{\mu}$ 's (right) at the NOvA near detector.

Appendix B.1.1). This was not a feature previously supported in CAFAna, thus was developed specifically for this analysis.

For the joint fit, we fit (or minimize $-2 \ln \lambda$ for) both oscillation channels at the same point in parameter space simultaneously and sum the resulting likelihoods values. In this way, we obtain an additional constraint from the other oscillation channel. Unlike oscillation analyses at the Far Detector, analyses at the Near Detector do not benefit 
from the cancellation of detector-correlated systematics. The joint fit allows us to regain some of the power of such correlations by considering systematics that is correlated between the selected $\nu_{e}$ and $\nu_{\mu}$ samples.

The $3+1$ model oscillation probabilities are dominantly dependent on $\Delta \mathrm{m}_{41}^{2}, \theta_{14}$ and $\theta_{24}$ as you can see in the SBL approximation shown in Equations (1.48) and (1.50). Because the three-flavor oscillations will have no noticeable effects at the Near Detector baseline length, the 3-flavor oscillation parameters used will not affect this analysis.

Since the majority of our sensitivity to the sterile mixing angles $\theta_{14}$ and $\theta_{24}$ comes from the appearance and disappearance channels, respectively, we also produce sensitivities to each mixing angles independently.

Furthermore, we produce sensitivities to $\theta_{\mu e}$ to compare to current limits measured by other SBL experiments. In order to calculate our sensitivity with respect to $\left(\Delta \mathrm{m}_{41}^{2}\right.$, $\left.\sin ^{2} 2 \theta_{14}\right)$ we profile over $\theta_{24}$ and $\theta_{34}$. To calculate our sensitivity with respect to $\left(\Delta \mathrm{m}_{41}^{2}\right.$, $\left.\sin ^{2} \theta_{24}\right)$ we profile over $\theta_{34}$ and $\theta_{14}$. To produce the sensitivities to $\left(\Delta \mathrm{m}_{41}^{2}, \sin ^{2} 2 \theta_{\mu e}\right)$ we profile over $\sin ^{2} 2 \theta_{14}, \sin ^{2} \theta_{24}$, and $\theta_{34}$.

We use the full $(3+1)$ model using the 'OscCalculatorSterile' calculator in making these sensitivities.

\subsection{New Tools Developed For SBL Analysis}

CAFAna is a collection of classes to perform any oscillation analysis in the NOvA experiment [126]. CAFAna uses Common Analysis Format (CAF) files as input. CAF files are lightweight and incorporate all the necessary information needed for the main analyses.

For this analysis, two new tools were needed. First was additions to CAFAna to treat SBL oscillations correctly, as covered in Section 6.2.1, and second was the development of a sample of appeared $\nu_{e}$ at the Near Detector, as described in Section 6.2.2.

\subsubsection{CAF additions}

The CAFAna framework needed to be expanded to include the necessary tools used to perform any SBL sterile analyses. All the new tools exist in a special feature branch, T17-10-10-sblana-br and subsequently R18-01-22-ndosc, that will be merged into trunk in due course. The tools that were implemented are briefly explained in the following sections and covered in detail in Appendix B. 
Specifically, we added functionality to CAFAna to allow for the treatment of neutrino oscillations in the Near Detector, where the oscillation probabilities depend on the variation in the baseline length $L$ of the neutrinos.

\section{- NDOscCurve}

This CAFAna class provides the oscillation probability histogram binned as a function of $L / E$. It is similar in design and functionality to the OscCurve class that is used to apply oscillations at the FD in the standard CAFAna framework, where the oscillation probability is binned as a function of simulated energy $E$ at a fixed $810 \mathrm{~km}$ baseline.

\section{- NDOscillatableSpectrum}

The NDOscillatableSpectrum class provides support for creating CAFAna spectra with true $L / E$ information. It functions in the same way as the OscillatableSpectrum class that is used for 3-flavor oscillation analyses at the Far Detector where the Y-axis represents simply the simulated neutrino energy due to the fixed baseline assumption.

\section{- NDEXTRAP}

This is a Near Detector-only oscillation analysis, so there is no actual extrapolation involved in the analysis. The NDExtrap class provides an interface that allows for design consistency with the current CAFAna framework. Rather than extrapolating components, this class takes ownership of NDOscillatableSpectrum objects for each selected component so that they can be used in a prediction class to apply oscillations.

\section{- NDPREDICTIONEXTRAP}

NDPredictionExtrap is a class for obtaining the predicted event rates after oscillations are applied to every selected component. This object can return the total predicted event rate or individual predicted rate for each component. Examples include beam components $\nu_{e} \mathrm{CC}, \nu_{\mu} \mathrm{CC}$, Neutral Current etc. oscillated with $\nu_{e} \rightarrow \nu_{e}, \nu_{\mu} \rightarrow \nu_{\mu}$, NC oscillations respectively.

\section{- Predictioninterpjoint Ana}

PredictionInterpJointAna is the key class for this analysis. It has very rich functionality as it does multiple things at a time. This object holds the systematically shifted prediction objects for every type of systematic uncertainty considered. It can return the predicted event rate for any systematic shift using the concept called "Cubic Interpolation" [127]. This is slightly different from what is used for 3-flavor oscillation analysis. 
As this analysis fits for $\nu_{\mu}$ disappearance and $\nu_{e}$ appearance simultaneously we need to correctly treat correlated and uncorrelated systematics. We use a method that is very similar to what was used by the 20173 -flavor joint $\nu_{e}-\nu_{\mu}$ analysis, but that was developed in parallel.

\subsubsection{Overlaid ND $\nu_{e}$ Appearance Sample}

For the standard FD oscillation analyses, the appeared $\nu_{e}$ is a fluxswap file where all $\nu_{\mu}$ events are swapped with $\nu_{e}$ events before they interact, therefore creating a file of $\nu_{e}$ events with the flux of $\nu_{\mu}$ events. As there is only ever one neutrino per spill at the FD, this method works. But at the ND where there are many neutrino interactions per spill fluxswap files create a very unrealistic situation in the detector as they change all muons in the files to electrons. Therefore resulting in an unrealistic spill of just electrons, instead of a spill with one electron and several muons.

To create a sample with a more realistic background composition, and hence reduce slicing effects in energy or efficiency due to background contamination, we generate a sample where we overlay single flux swapped CC $\nu_{e}$ 's events on top of standard ND Production 3 files. A detailed explanation of the process of overlaying can be found in Refs. $[128,129]$. After the $\nu_{e}$ 's are overlaid, we reconstruct those events following the standard reconstruction procedure. This sample which adds $1 \nu_{e}$ per event is equivalent to having MC generated at slightly higher POT/spill than the standard MC. To account for any reconstruction efficiency differences due to this, we study differences in the selected efficiency of the beam $\nu_{e}$ and these overlaid appeared $\nu_{e}$ events as described in our systematic tech note [130].

\subsection{Datasets}

This analysis will use the NuMI ND data collected from October 2014 to May 2017 corresponding to $8.05 \times 10^{20}$ POT (2017 analysis including Periods 1, 2, 3, and 5) and use the standard Production $3 \mathrm{MC}$ samples. We also use the standard PPFX weights (kPPFXFluxCVWgt) [119] and cross-section weights (kXSecCVWgt2018) [131]. The following are the datasets used for this analysis:

\section{- NuMI Data}

prod_caf_R17-03-01-prod3reco.d_nd_numi_fhc_full_v1_goodruns

\section{- Non swap}

prod_caf_R17-03-01-prod3reco.d_nd_genie_nonswap_fhc_nova_v08_full_v1 


\section{- Overlay}

prod_caf_R17-03-01-prod3reco.h_nd_genie_nonswap_fhc_nova_v08_full_nuecc-overlay_v1 The systematic samples used are described in the accompanying systematic tech note.

\subsection{Event Selection}

This analysis will be doing a joint fit of appeared $\nu_{e}$ 's and disappeared $\nu_{\mu}$ 's, so we will select two samples, 1) $\nu_{e}$ sample and 2) $\nu_{\mu}$ sample.

We apply a selection very similar to the second analysis ND $\nu_{e}$ [132], [133] and $\nu_{\mu}$ [134], [135] selections developed by the collaboration for three-flavor neutrino oscillation analysis with a few small changes. For the $\nu_{e}$ selection, we use a tighter selection on CVNe to reduce backgrounds and loosen the $\nu_{e}$ energy range to $1-5 \mathrm{GeV}$. For the $\nu_{\mu}$ selection we use CVNm for selecting $\nu_{\mu}$ 's in this analysis but keep other cuts the same as the second analysis.

Since the ND data is our signal in this analysis, we do not include the standard ND data-MC comparison plots. To ensure that the selections are well behaved we do check a small, blinded sample as explained in Section 6.9.2.

\subsubsection{Beam and Quality cuts}

For both the $\nu_{e}$ sample and $\nu_{\mu}$ sample spill cuts are applied on a spill-by-spill basis to ensure that the beam conditions are good and that the detector is functioning well.

\section{Beam Quality Cuts[136]:}

- The time to the nearest beam spill in the database (deltaspilltimensec) must be less than $0.5 \mathrm{~s}$.

- The POT in the spill must exceed $2 \times 10^{12}$.

- The horn current must be between -202 kA and -198 kA

- The beam $\mathrm{x}$ and y positions must be between $-2.00 \mathrm{~mm}$ and $2.00 \mathrm{~mm}$.

- The beam $\mathrm{x}$ and $\mathrm{y}$ widths must be between $0.57 \mathrm{~mm}$ and $1.58 \mathrm{~mm}$. 
These cuts are not applied to MC as all MC is simulated with perfect beam conditions.

\section{Data Quality Cuts[137]:}

- The fraction of hits in the top DCMs must be $<45 \%$ to reject events caused by the lights in the detector hall.

- No DCMs can be missing from the run.

The detailed implementation of these cuts can be found in "CAFAna/Cuts/SpillCuts.h"

\subsection{2 $\nu_{e}$ Selection Cuts}

A series of cuts explained in detail in [138] are applied to each slice to ensure that it is well reconstructed and that the event is likely to be well contained in the detector.

\section{Data quality and Reconstruction:}

- The number of hits in the slice divided by the number of planes must be less than eight, to reject "flasher" events.

- The event must have an ElasticArms vertex, with at least one LID "shower" [139].

- The leading LID "shower" is required to have at least five hits in each view and the gap between the primary shower and ElasticArms vertex must be less than $100 \mathrm{~cm}$.

- We require that at least $70 \%$ of the hits in a slice belong to some combination of the $3 \mathrm{D}$ showers in the slices.

- We cut events with asymmetric leading showers, such that we require that neither the $\mathrm{x}$ or $\mathrm{y}$-view have more than $60 \%$ of the showers hits.

- For events with at least two showers, we require that the cosine of the angle between the highest energy showers be greater than -0.95 .

Fiducial: The ElasticArms vertex must lie within $+160 \mathrm{~cm}$ and $-100 \mathrm{~cm}$ of the detector center in $\mathrm{x},+100 \mathrm{~cm}$ and $-160 \mathrm{~cm}$ in $\mathrm{y}$, and have $100 \mathrm{~cm}<\mathrm{z}<900 \mathrm{~cm}$.

Containment: The start and stop positions of all LID "showers" must be within 
$\pm 170 \mathrm{~cm}$ of the centerline in $\mathrm{x}$ and $\mathrm{y}$, and beyond $100 \mathrm{~cm}$ and before $1225 \mathrm{~cm}$ in $\mathrm{z}$.

Front planes: There must be six or more planes with no hits before the most upstream hit in the slice.

Slice hits: The slice must have between 21 and 199 hits inclusive.

$\nu_{e}$ energy: The $\nu_{e}$ energy must have less than $5 \mathrm{GeV}$ total reconstructed energy.

Shower length: The primary FuzzyK prong must be between $140 \mathrm{~cm}$ and $500 \mathrm{~cm}$ in length.

ND $\nu_{e}$ PID We apply CVNe [115] PID. We select events which have a CVNe output greater than 0.95 , only using the highest purity bin.

Transverse momentum: We requiring that $\mathrm{pT} / \mathrm{p}$ be greater than 0.65 . Transverse momentum calculated here using LID "showers".

The fraction of events passing each of these cuts, in turn, are shown in Tables 6.1 and 6.2 .

\subsection{3 $\nu_{\mu}$ Selection Cuts}

Three cut levels, explained in detail in Ref. [135], are used to select $\nu_{\mu}$ interactions: Quality, Containment and $\nu_{\mu}$ CC PID.

\section{Event Quality:}

- Existence of at least one 3D Kalman track

- More than 20 hits in the slice

- more than four planes span for a slice

\section{Containment:}

- Number of cells from the edge is more than one,

- first plane in the slice is 2 or more,

- last plane in the slice is 212 or less,

- primary track starts in an active region and stops before $\mathrm{Z}=1275 \mathrm{~cm}$,

- hadronic calorimetric energy in the muon catcher is less than $30 \mathrm{MeV}$,

- there must be more than four cells before we hit the detector edge from the track end in the forward direction and more than eight cells from the track end in the backward direction in the primary track. 
$\nu_{\mu}$ CC PID: The $\nu_{\mu}$ classifier output of CVN, CVNm, is used to select $\nu_{\mu}$. The output score for CVNm must be more than 0.5 [115].

\subsection{Selected events}

The event rates after each type of selection is applied are shown in Table 6.1 for the selected beam $\nu_{e}$ and Table 6.2 for the selected $\nu_{\mu}$. All the rates are normalized to $8.05 \times 10^{20}$ POT.

The distribution of events for both the beam $\nu_{e}$ and $\nu_{\mu}$ samples after the selection described in Section 6.4 and using the energy estimators derived in Section ?? are given in Figure 6.6.

\begin{tabular}{c|c|c|c|c|c} 
Selection & Total MC & Efficiency $(\%)$ & $\nu_{\mu}$ & $\nu_{e}$ & $\mathrm{NC}$ \\
\hline No Cut & $1.106 \mathrm{e}+08$ & - & $9.654 \mathrm{e}+07$ & $1.281 \mathrm{e}+06$ & $1.283 \mathrm{e}+07$ \\
\hline Data Quality & $7.622 \mathrm{e}+07$ & - & $6.985 \mathrm{e}+07$ & $6.24 \mathrm{e}+05$ & $5.397 \mathrm{e}+06$ \\
\hline Fiducial & $5.142 \mathrm{e}+06$ & - & $4.225 \mathrm{e}+06$ & $7.503 \mathrm{e}+04$ & $8.291 \mathrm{e}+05$ \\
\hline Containment & $1.27 \mathrm{e}+06$ & 39.79 & $8.323 \mathrm{e}+05$ & $2.893 \mathrm{e}+04$ & $4.012 \mathrm{e}+05$ \\
\hline FrontPlanes & $1.262 \mathrm{e}+06$ & 39.56 & $8.263 \mathrm{e}+05$ & $2.876 \mathrm{e}+04$ & $3.992 \mathrm{e}+05$ \\
\hline \# Hits & $1.185 \mathrm{e}+06$ & 32.76 & $8.04 \mathrm{e}+05$ & $2.382 \mathrm{e}+04$ & $3.526 \mathrm{e}+05$ \\
\hline Energy & $1.137 \mathrm{e}+06$ & 26.26 & $7.758 \mathrm{e}+05$ & $1.909 \mathrm{e}+04$ & $3.383 \mathrm{e}+05$ \\
\hline ProngLength & $7.738 \mathrm{e}+05$ & 25.17 & $5.135 \mathrm{e}+05$ & $1.83 \mathrm{e}+04$ & $2.399 \mathrm{e}+05$ \\
\hline Ptp & $6.677 \mathrm{e}+05$ & 23.63 & $4.523 \mathrm{e}+05$ & $1.718 \mathrm{e}+04$ & $1.968 \mathrm{e}+05$ \\
\hline CVN >0.95 & 9512 & 9.63 & 1242 & 7001 & 1269 \\
\hline
\end{tabular}

TABLE 6.1: Table shows the cut flow for the selected events using non swap sample using the $\nu_{e}$ selection with no oscillation. Here, efficiency $=\nu_{e}$ 's selected with full selection $/ \nu_{e}$ 's having true vertex in the fiducial volume. These numbers are corresponding to a data POT $8.05 \mathrm{e}+20$.

\begin{tabular}{c|c|c|c|c|c} 
Selection & Total MC & Efficiency $(\%)$ & $\nu_{\mu}$ & $\nu_{e}$ & $\mathrm{NC}$ \\
\hline No Cut & $1.11 \mathrm{e}+08$ & - & $9.65 \mathrm{e}+07$ & $1.28 \mathrm{e}+06$ & $1.28 \mathrm{e}+07$ \\
\hline Data Quality & $8.506 \mathrm{e}+07$ & - & $7.785 \mathrm{e}+07$ & $6.267 \mathrm{e}+05$ & $6.255 \mathrm{e}+06$ \\
\hline Containment & $4.554 \mathrm{e}+06$ & - & $3.227 \mathrm{e}+06$ & $5.444 \mathrm{e}+04$ & $1.252 \mathrm{e}+06$ \\
\hline CVN $>0.5$ & $2.508 \mathrm{e}+06$ & 76.75 & $2.477 \mathrm{e}+06$ & 571.8 & $3.014 \mathrm{e}+04$ \\
\hline
\end{tabular}

TABLE 6.2: Table shows the cut flow for the selected events using non swap sample using the $\nu_{\mu}$ selection with no oscillation. Here, efficiency is defined as $\nu_{\mu}$ 's selected with full selection $/ \nu_{\mu}$ 's which are contained. These numbers are corresponding to a data POT $8.05 \mathrm{e}+20$. 

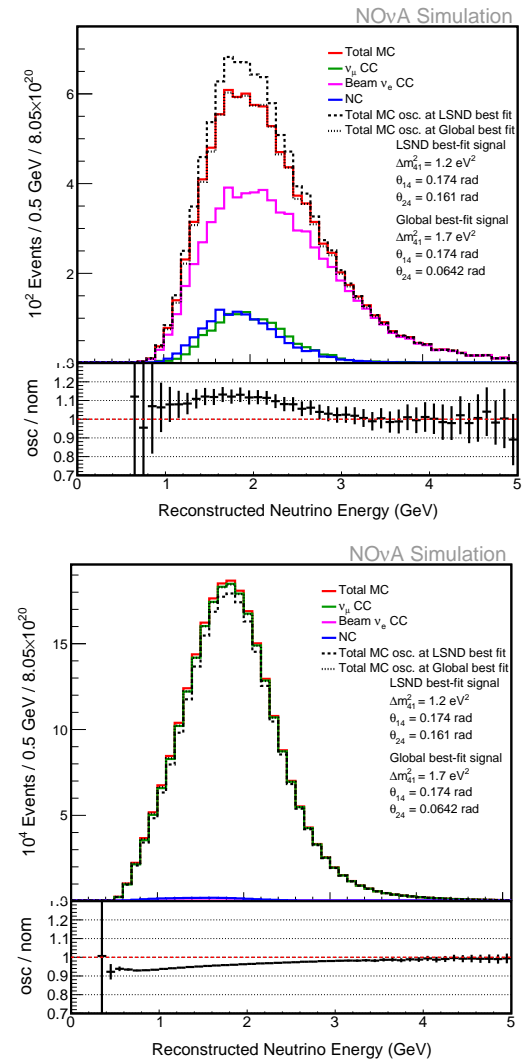

FIGURE 6.6: Showing the distribution of reconstructed energy after the $\nu_{e}$ (top) and $\nu_{\mu}$ (bottom) selection applied. The dotted line indicates the total prediction in a $3+1$ model using the oscillation parameters shown on the plot. To use the LSND limits which are provided in $\theta_{\mu e}$, we set $\theta_{14}$ to 10 degrees driven by the Bugey limits on this angle. The solid lines show the 3-flavor prediction, the dotted at the 2017 Global fit best fit, and the dashed at the LSND best fit.

\subsubsection{Expected signal}

The amount of signal we expect to see at the Near Detector obviously depends on the oscillation parameters. In Table ??, we show the event rates assuming no oscillations, at the LSND best fit, and at the global best-fit oscillation parameters in turn.

\begin{tabular}{|c|c|c|c|c|c|}
\hline & Appeared $\nu_{e}$ & Total Prediction & $\nu_{\mu}$ & beam $\nu_{e}$ & $\mathrm{NC}$ \\
\hline Unoscillated & 0 & 9511.34 & 1241.67 & 7000.83 & 1268.84 \\
\hline LSND best fit & 1028.1 & 10259.5 & 1202.59 & 6783.92 & 1244.9 \\
\hline Global best fit & 278.862 & 9403.34 & 1230.59 & 6632.56 & 1261.34 \\
\hline
\end{tabular}

TABLE 6.3: This table shows the event rates expected for $\nu_{e}$ appearance channel at the LSND best fit point $\left(\sin ^{2} 2 \theta, \Delta \mathrm{m}^{2}\right)=\left(0.003,1.2 \mathrm{eV}^{2}\right)$ and the global best-fit $\left(\sin ^{2} 2 \theta, \Delta \mathrm{m}^{2}\right)=\left(0.00048,1.7 \mathrm{eV}^{2}\right)[87,88]$ oscillation parameters. 


\begin{tabular}{|c|c|c|c|c|c|}
\hline & Appeared $\nu_{e}$ & Total Prediction & $\nu_{\mu}$ & beam $\nu_{e}$ & NC \\
\hline Unoscillated & 0 & $2.50473 \mathrm{e}+06$ & $2.47404 \mathrm{e}+06$ & 573.899 & 30118.8 \\
\hline LSND best fit & 71.7224 & $2.40699 \mathrm{e}+06$ & $2.37686 \mathrm{e}+06$ & 555.38 & 29503.7 \\
\hline Global best fit & 18.7346 & $2.47915 \mathrm{e}+06$ & $2.44866 \mathrm{e}+06$ & 545.039 & 29930.8 \\
\hline
\end{tabular}

TABLE 6.4: This table shows the event rates expected for $\nu_{\mu}$ appearance channel at the LSND best fit point $\left(\sin ^{2} 2 \theta, \Delta \mathrm{m}^{2}\right)=\left(0.003,1.2 \mathrm{eV}^{2}\right)$ and the global best-fit $\left(\sin ^{2} 2 \theta, \Delta \mathrm{m}^{2}\right)=\left(0.00048,1.7 \mathrm{eV}^{2}\right)[87,88]$ oscillation parameters.

\subsection{Details of fitting framework}

Sensitivities are produced with respect to $\left(\Delta \mathrm{m}_{41}^{2}, \sin ^{2} 2 \theta_{14}\right)$ while profiling over $\theta_{24}$, to $\left(\Delta \mathrm{m}_{41}^{2}, \sin ^{2} \theta_{24}\right)$ profiling over $\theta_{34}$ and $\theta_{14}$, and to $\left(\Delta \mathrm{m}_{41}^{2}, \sin ^{2} \theta_{\mu e}\right)$ profiling over $\theta_{24}$, $\theta_{34}$ and $\theta_{14}$. $\operatorname{Sin}^{2} 2 \theta_{14}$ does not depend on $\theta_{34}$, so we don't profile it while making that sensitivity. The energy distribution being fitted has one hundred bins from 0 to $10 \mathrm{GeV}$.

\subsubsection{Log-Likelihood Fitting}

In any neutrino oscillation analysis, predicted or expected event rates are fitted with either the experimental data or the fake data made from Monte Carlo. For this analysis, we use the log-likelihood method of fitting for the binned data.

$$
-2 \ln \lambda=2 \sum\left[\mu-n+n \ln \frac{n}{\mu}\right]
$$

where $\lambda$ is the likelihood function, $\mu$ is the expected event count and $n$ is the observed event count.

\subsubsection{Joint fit versus Single fit}

To increase the sensitivity of an experimental result, instead of doing a single fit with one particular channel, one can combine two channels (of the selected $\nu_{e}$ and $\nu_{\mu}$ ) which we will call the joint fit method. This method gives more power either to determine the true parameter value or to place a stronger constraint on the parameter and to constrain systematic uncertainties. 
To demonstrate the power of joint analysis, in Fig. 6.7, we compare the sensitivities on $\left(\Delta \mathrm{m}_{41}^{2}, \sin ^{2} 2 \theta_{14}\right)$ from both the single and joint analysis methods. The single method uses the $\nu_{e}$ appearance channel only and the joint fit uses both the $\nu_{e}$ appearance and $\nu_{\mu}$ disappearance channels. The $\nu_{\mu}$ disappearance channel on its own is not sensitive to $\sin ^{2} 2 \theta_{14}$ so is not shown. You can see the improvements in the $90 \%$ CL sensitivities (region to the right of curves is excluded) using the joint fit.

The formalism used to combine several measurements by performing a joint maximumlikelihood fit of different datasets is:

$$
\mathcal{L}_{\text {Joint }}^{i, j}=\mathcal{L}_{A}^{i, j}+\mathcal{L}_{B}^{i, j}
$$

where $\mathcal{L}_{\text {Joint }}^{i, j}$ is the value of log-likelihood for a combined experiment at $(i, j)^{\text {th }}$ bin in the parameter space. $A$ and $B$ are individual experiments and $\mathcal{L}_{A, B}^{i, j}$ is log-likelihood from the experiment $A$ and $B$.

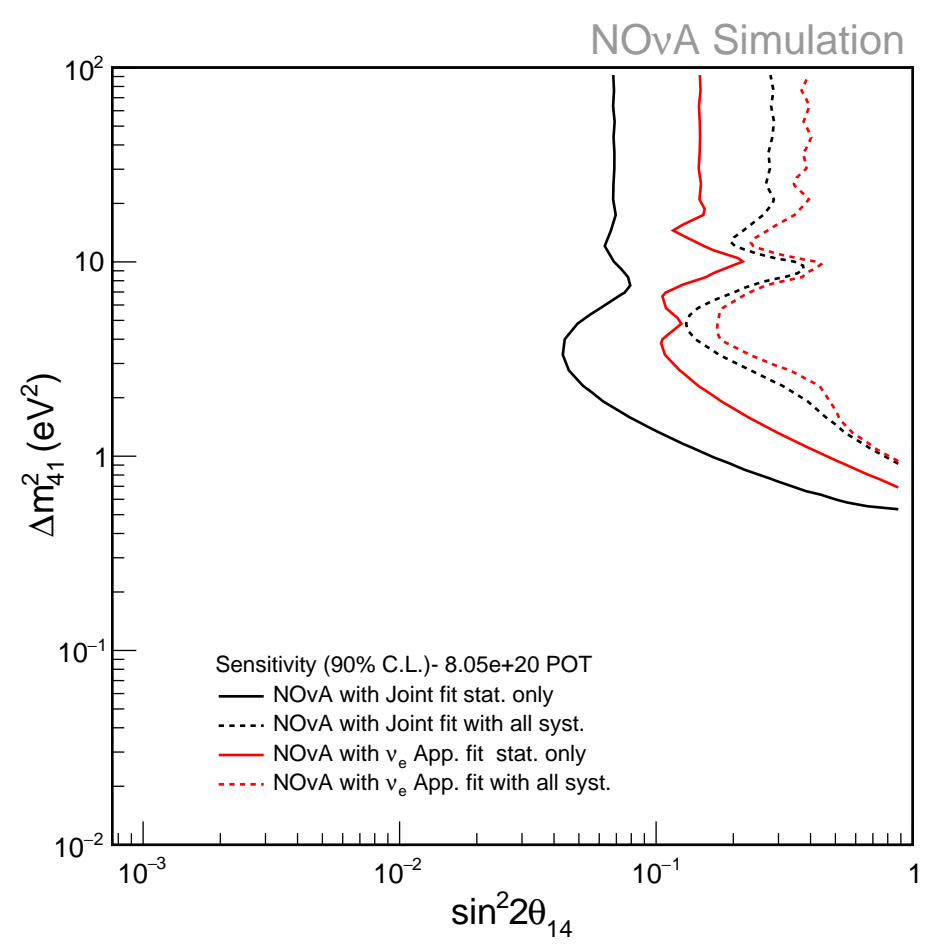

Figure 6.7: Comparison of $\sin ^{2} \theta_{14}$ Vs $\Delta m_{41}^{2}$ sensitivity between single fit (red) and joint fit (black) with systematics (dashed) and without systematics (solid).

\subsection{Analysis Deliverables}

If sterile neutrinos exist, we expect $\nu_{\mu}$ 's to disappear and $\nu_{e}$ 's to appear via shortbaseline oscillations through sterile states. The $3+1$ model oscillation probabilities for 
$\nu_{\mu}$ disappearance and $\nu_{e}$ appearance share the same functional dependence on $\theta_{24}$, this will help us to constrain $\theta_{24}$ very well with the joint analysis. We made the sensitivity in terms of $\Delta \mathrm{m}_{41}^{2}$ and all of $\theta_{14}, \theta_{24}$, and $\theta_{\mu e}$. The mixing angle $\theta_{14}$ is sensitive to $\nu_{e}$ appearance, $\theta_{24}$ is sensitive to $\nu_{\mu}$ disappearance, and $\theta_{\mu e}=\sin ^{2} 2 \theta_{14} \sin ^{2} \theta_{24}$ allows us to compare to previous SBL limits. Making sensitivities to all three angles allows us to have a complete picture as possible.

\subsubsection{0\% CL limits $\theta_{14}$ as a function of $\Delta \mathbf{m}_{41}^{2}$}

The selected $\nu_{e}$ sample where we search for $\nu_{e}$ appearance gives us most of our sensitivity to $\theta_{14}$. The strongest limits on $\theta_{14}$ are currently from reactor experiments which are able to measure $\theta_{14}$ through $\bar{\nu}_{e}$ disappearance, such as the Bugey limits shown on Fig. 6.8.

As shown in Eq.1.48 the $\nu_{e}$ appearance probability depends on $\theta_{14}$ as $\sin ^{2} 2 \theta_{14}$. The 90\% C.L. sensitivity to $\sin ^{2} 2 \theta_{14}$ is shown in Fig. 6.8 for the joint fit method between $\nu_{e}$ appearance and $\nu_{\mu}$ disappearance samples.

To determine this sensitivity, the three-flavor oscillation parameters are fixed (as they do not affect ND oscillation), and we profile over $\theta_{24}$ and $\theta_{34}$. Systematics are included into the fit by profiling over the various systematics, as described in detail in Ref. [130].

After including all the systematics, NOvA does not exclude any region of $\theta_{14}$ not already excluded.

\subsubsection{0\% CL limits $\theta_{24}$ as a function of $\Delta \mathbf{m}_{41}^{2}$}

The selected $\nu_{\mu}$ sample where we search for $\nu_{\mu}$ disappearance gives us most of our sensitivity to $\theta_{24}$. The NOvA Near Detector also has a sensitivity to the $\nu_{\mu}$ disappearance. The $\nu_{\mu}$ disappearance probabilities depend on the mixing angles $\theta_{24}, \theta_{14}$, and $\theta_{34}$, with most of the power coming from $\theta_{24}$.

To make the $\theta_{24}$ sensitivity as shown in Fig. 6.9 we profile over $\theta_{34}$ and $\theta_{14}$. The $90 \%$ CL sensitivity with and without systematics is compared to experimental results from CDHS[140], CCFR[141], and SciBooNE[142] plus MiniBooNE[77]. We observe that NOvA constrains $\theta_{24}$ well in the region $\Delta m_{41}^{2}>0.2 \mathrm{eV}^{2}$. Not shown on this Figure are limits from LBL experiments i.e. MINOS and Icecube which place strong limits on $\Delta m_{41}^{2}<10 \mathrm{eV}^{2}$. 


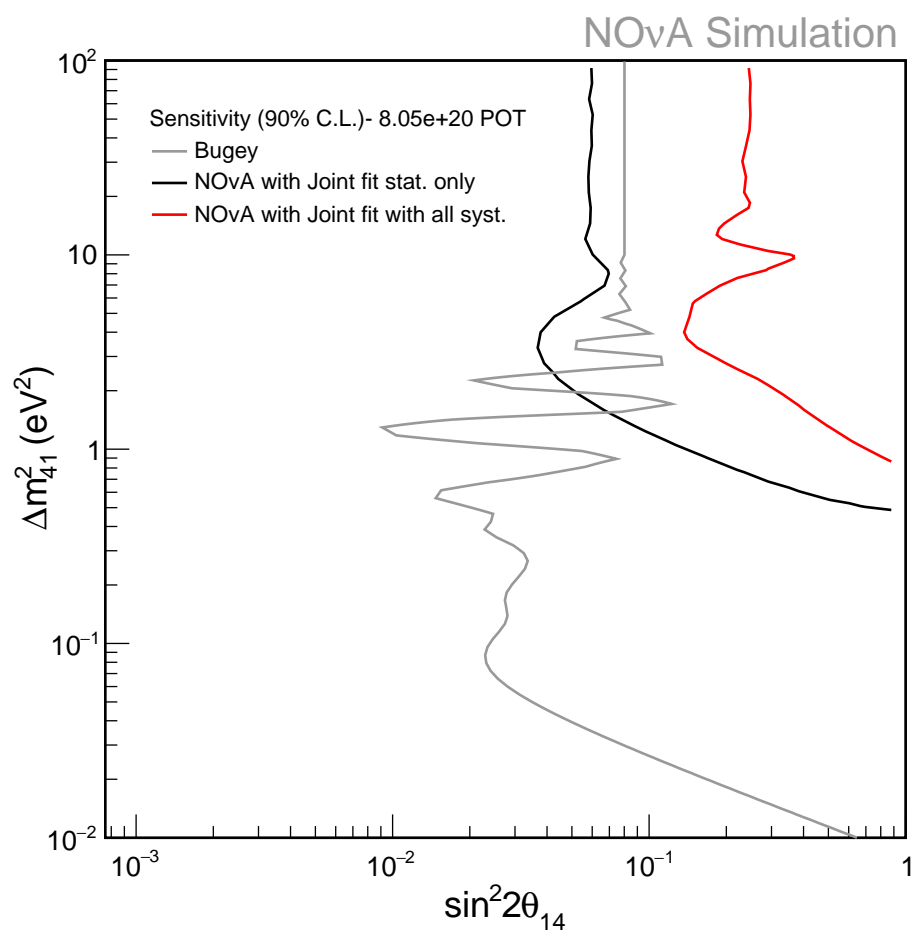

FiguRE 6.8: Showing the limits for $\theta_{14}$ in $\sin ^{2} 2 \theta_{14}$ Vs $\Delta m_{41}^{2}$ surface, while profiling over $\theta_{24}$ We also compare the $\theta_{14}$ limits with Bugey results at 90\% C.L.

\subsubsection{Sensitivity for $\theta_{\mu e}$ as a function of $\Delta \mathbf{m}_{41}^{2}$}

The sensitivity for the mixing angle $\theta_{\mu e}$ using the joint fit method is shown in Fig.6.10. We perform the fit for both $\nu_{e}$ appearance and $\nu_{\mu}$ disappearance simultaneously to set the limits on $\theta_{\mu e}$. The mechanism of joint fit is explained in Sec. 6.6. We profile over $\theta_{34}$, $\theta_{24}$, and $\theta_{14}$ in making these limits. We observe that for $8.05 \mathrm{e}+20$ POT, our sensitivity shows we can place strong limits comparable with those from MiniBooNE.

\subsection{Mock Data Challenges}

We performed a series of mock data challenges to validate the framework and check our confidence in the interpretation of experimental results. We do these in two separate ways as described in this section.

\subsubsection{Mock Data Sensitivity with no signal injection}

We want to determine that the analysis will not interpret statistical fluctuations as a sterile signal. In determining the sensitivities as shown in Section 6.7, we fit the 


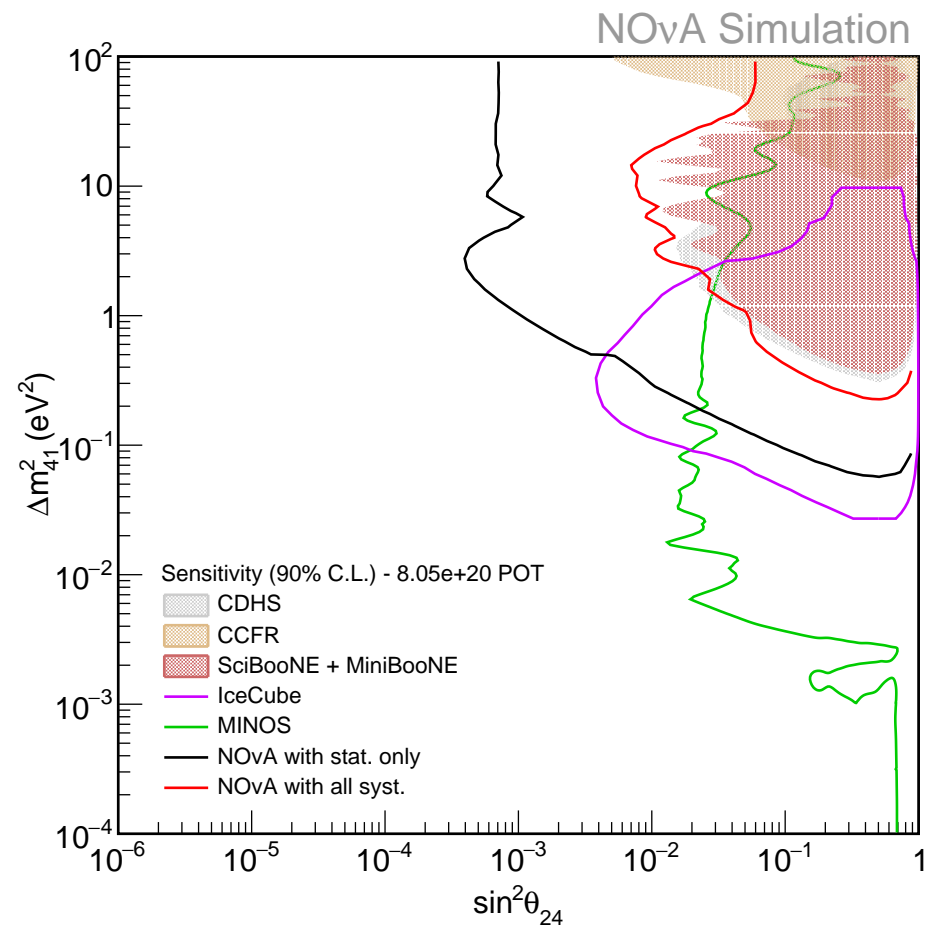

Figure 6.9: Sensitivity for $\theta_{24}$ while profiling over $\theta_{34}$ and $\theta_{14}$ at $90 \%$ C.L. The regions excluded by CDHS, CCFR, MiniBooNE/SciBooNE are also shown.

prediction with fake data (by which we mean the prediction normalized to a specific POT). For the mock data challenge, we fit the prediction with mock data, which is fake data that also includes appropriate Poisson fluctuations in every bin. Figure 6.11 shows the nominal prediction and corresponding mock data. Figure 6.12 (right) shows the sensitivity for the mock data compared to the nominal sensitivity.

We are in the process of forming a 1 and 2 sigma band for our sensitivity, which should be available shortly. The sensitivities from 1000 mock data universes are also shown in Figure 6.12 (left). This mock data 'universe' should easily lie within the 2 sigma band. In the final result, we will include these 1 and 2 sigma bands in a plot similar to Figure 3 from Ref. [64].

\subsubsection{Mock Data Sensitivity with LSND signal injection}

A second test we did was was to inject a sterile signal and check that the framework responded as expected. In Fig. 6.13, we showed the energy distribution of nominal un-oscillated and oscillated predictions at LSND best fit mixing parameters. We made sensitivities by fitting the predictions with signal at LSND best fit $\left(\sin ^{2} 2 \theta, \Delta \mathrm{m}^{2}\right)=$ $\left(0.003,1.2 \mathrm{eV}^{2}\right)$. As shown in Figure 6.14 (red curve) we produced a closed contour at 


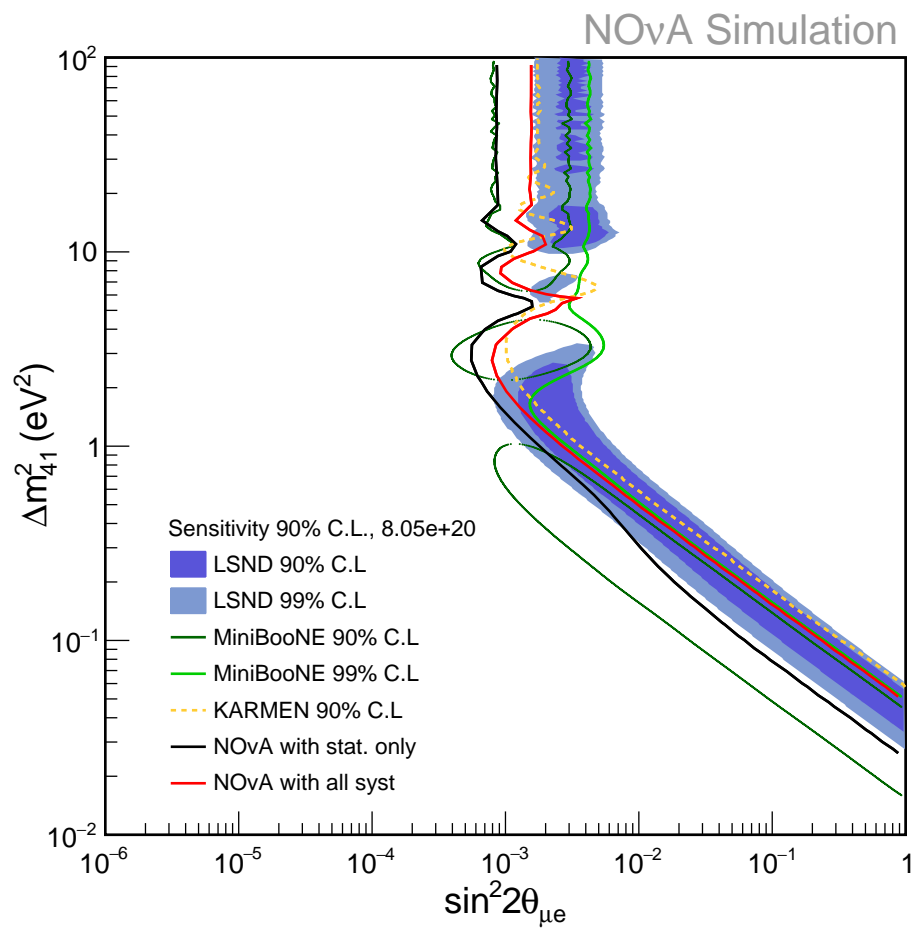

FiguRe 6.10: Sensitivity for $\theta_{\mu e}$ while profiling over $\theta_{34}$ at $90 \%$ C.L. for $8.05 \mathrm{e}+20$ POT. The regions excluded by LSND, MiniBooNE and KARMEN are also shown.
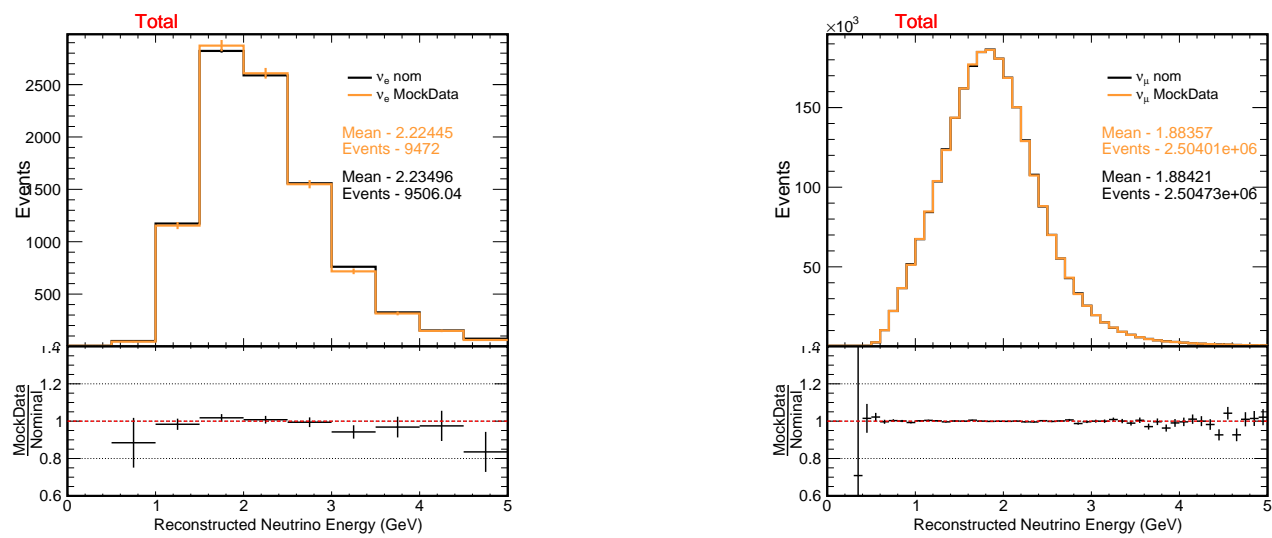

FIGURE 6.11: The distribution of reconstructed energy of $\nu_{e}$ selected events (left) and $\nu_{\mu}$ selected events with CVN (right) with the Poisson fluctuations applied in every energy bin.

the $\left(\Delta \mathrm{m}^{2} \sin ^{2} 2 \theta\right)$ of the injected signal. With the systematics (black), the sensitivity has a shape similar to that of LSND results.

This shows our framework behaves correctly as a sterile signal. 

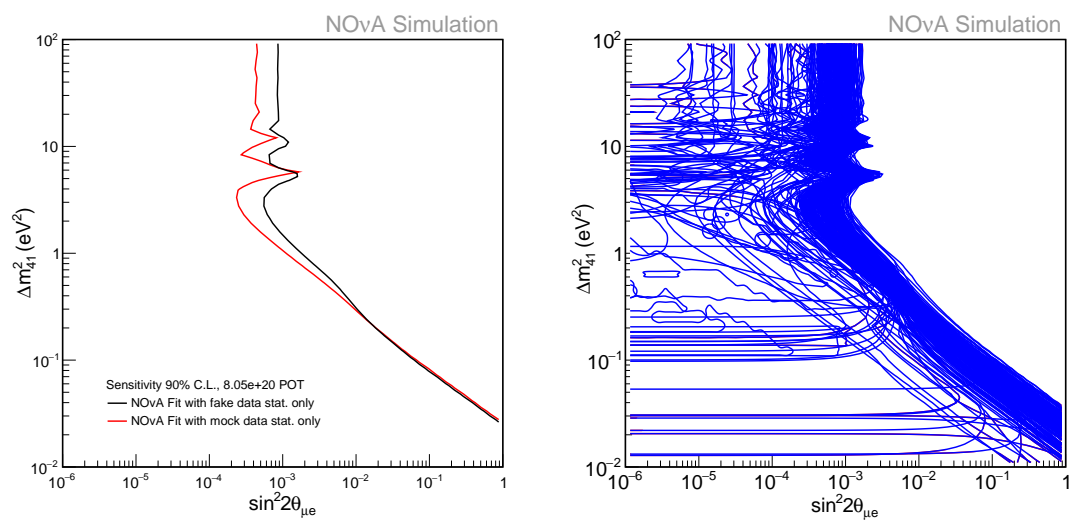

Figure 6.12: Left: The sensitivities after fitting with fake data (black) at $90 \%$ C.L., with mock data (solid red) at 90\% C.L. These sensitivities are made for 8.05e+20 POT. Right: 200 mock data universes with only statistical fluctuations.
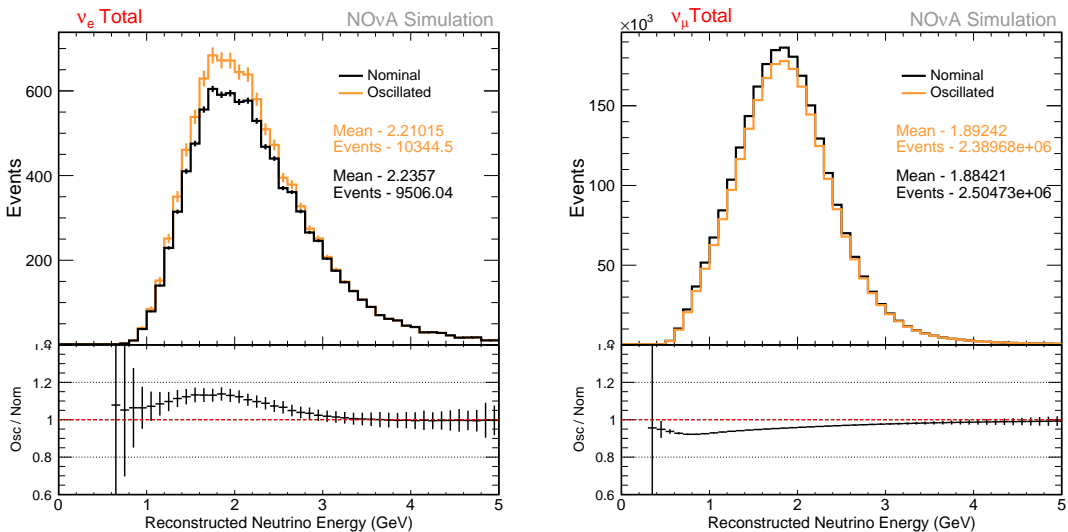

FiguRE 6.13: Show the reconstructed energy distribution of nominal unoscillated prediction and the expected oscillated prediction at LSND best fit mixing parameters for both $\nu_{e}$ (left) and $\nu_{\mu}$ selected sample.

\subsection{Studies using data}

This analysis uses the Near Detector data as its signal. Therefore, we don't look at the ND data after the selection as a function of energy, but we do a few cross checks, as covered in the section, to validate that things are ok.

\subsubsection{Near Detector Data and MC Comparison}

In this analysis, we also compare the distribution of reconstructed energy between Near Detector data and $\mathrm{MC}$ for the $\nu_{e}$ and $\nu_{\mu}$ selection, refer Section 6.4, except for the final PID selection. We observe both the shape and normalization differences between data and $\mathrm{MC}$ for both $\nu_{e}$ and $\nu_{\mu}$ selected samples, as shown in Fig. ??, which may be due to 


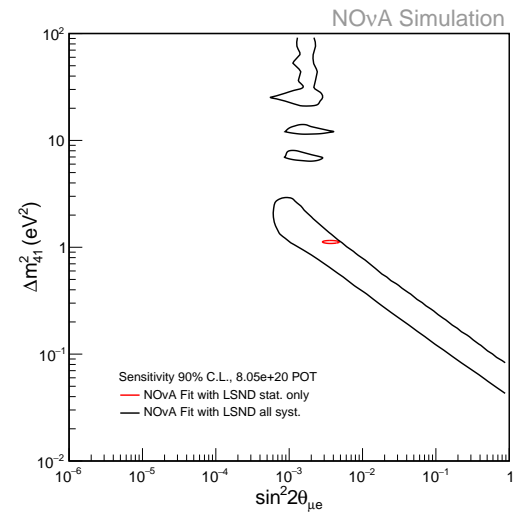

Figure 6.14: The sensitivities after fitting with LSND best fit signal injection with no systematics (red) at 90\% C.L., with all systematics (black) at 90\% C.L. These sensitivities are made for $8.05 \mathrm{e}+20$ POT.

cross-section, calibration and beam uncertainties, but as you can see from the Fig. ??, all these differences are covered with the systematic uncertainties. Here are the details of the study,

\section{- GENIE}

prod_caf_R17-03-01-prod3reco.d_nd_genie_nonswap_fhc_nova_v08_full_v1

- Data

prod_caf_R17-03-01-prod3reco.d_nd_numi_fhc_full_v1_goodruns

- Weights applied to MC- kPPFXFluxCVWgt, kXSecCVWgt2018

- Normalized to $8.05 \mathrm{e}+20$
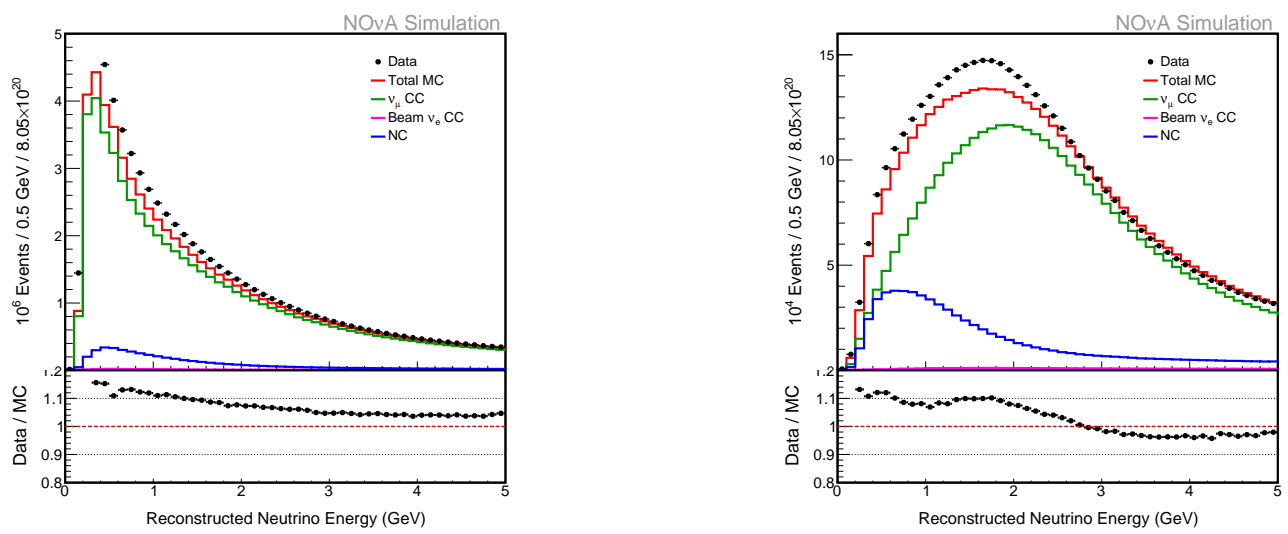

FIGURE 6.15: Shows the preselection distribution of reconstructed neutrino energy for the events which pass the $\nu_{e}$ data quality (left) and fiducial (right) selection. The MC is normalized to the data POT of $8.05 \mathrm{e}+20$. 

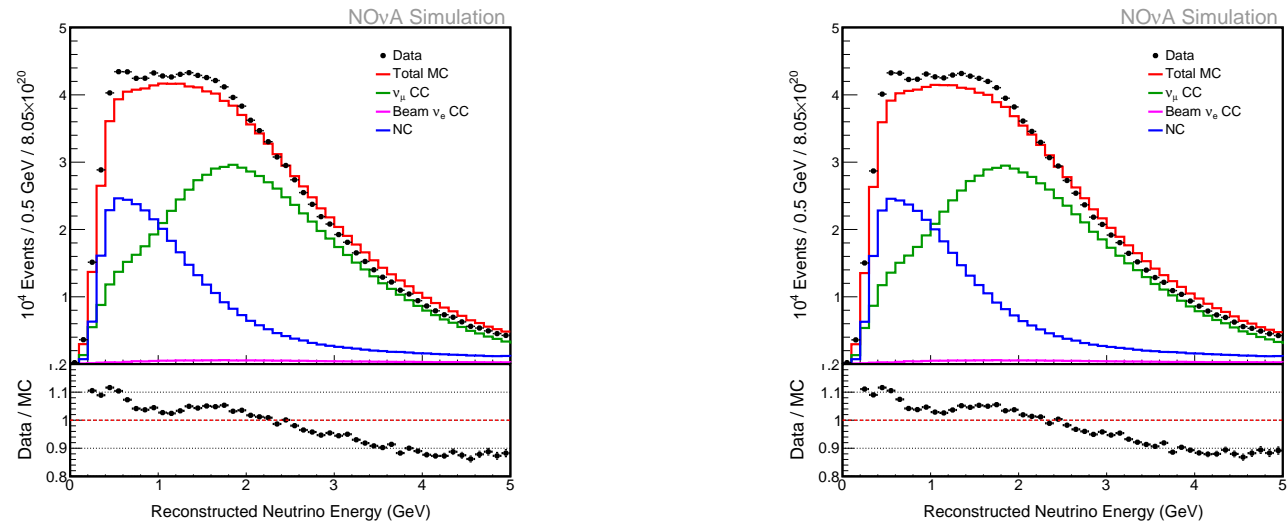

FiguRE 6.16: Shows the preselection distribution of reconstructed neutrino energy for the events which pass the $\nu_{e}$ containment (left) and front planes (right) selection. The $\mathrm{MC}$ is normalized to the data POT of $8.05 \mathrm{e}+20$.
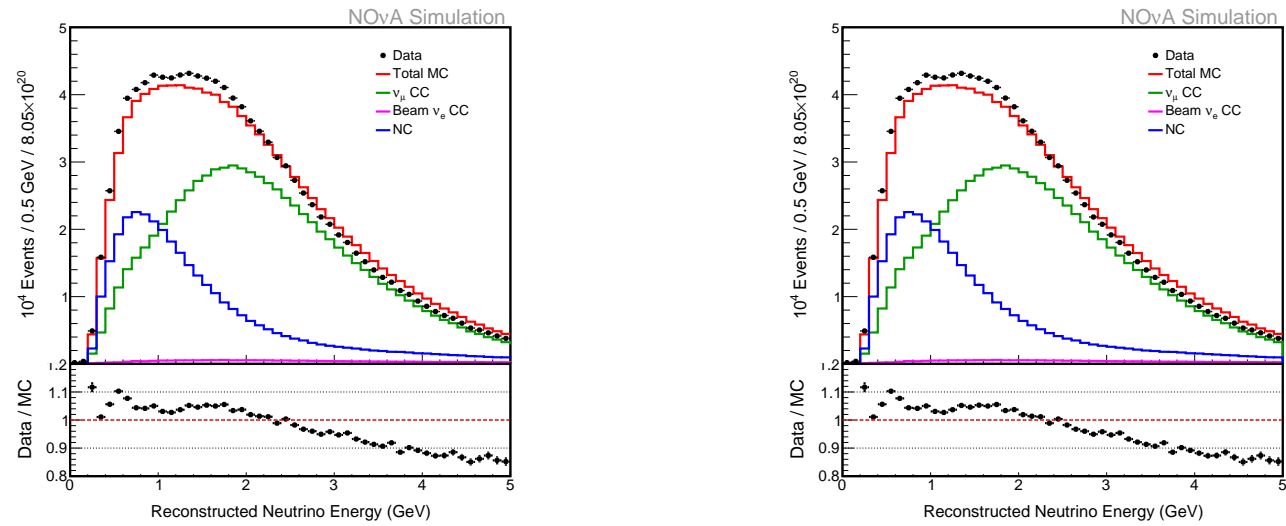

FiguRE 6.17: Shows the preselection distribution of reconstructed neutrino energy for the events which pass the $\nu_{e}$ number of hits (left) and energy (right) selection. The $\mathrm{MC}$ is normalized to the data POT of $8.05 \mathrm{e}+20$.

\subsubsection{Blinding Study}

We are looking for an oscillation signal, $\nu_{\mu} \rightarrow \nu_{e}$ and $\nu_{\mu} \rightarrow \nu_{\mu}$, in the near detector. In this study we look at variables which don't give any information about the energy of a neutrino interaction, so we remain blind to any potential signal. The variables chosen are those used in the preselection for the two samples. For the $\nu_{e}$ sample we looked at the number of vertices, the X, Y, Z location of vertices, the number of showers, and the X, Y, Z start and stop position of the showers. For the $\nu_{\mu}$ sample we looked at, the number of cells from the edge, the number of tracks, and the $\mathrm{X}, \mathrm{Y}, \mathrm{Z}$ start and stop position of the tracks. As we see from the distribution of various unblinded variables for both data and MC for $\nu_{e}$ shown in Fig. ??, and $\nu_{\mu}$ selected samples shown in in Fig. ??, the difference between the data and $\mathrm{MC}$ is about $11.5 \%$ for the $\nu_{e}$ sample. This is consistent with the data-MC difference observed by the $\nu_{e}$ group for the 2017 analysis [143] before 

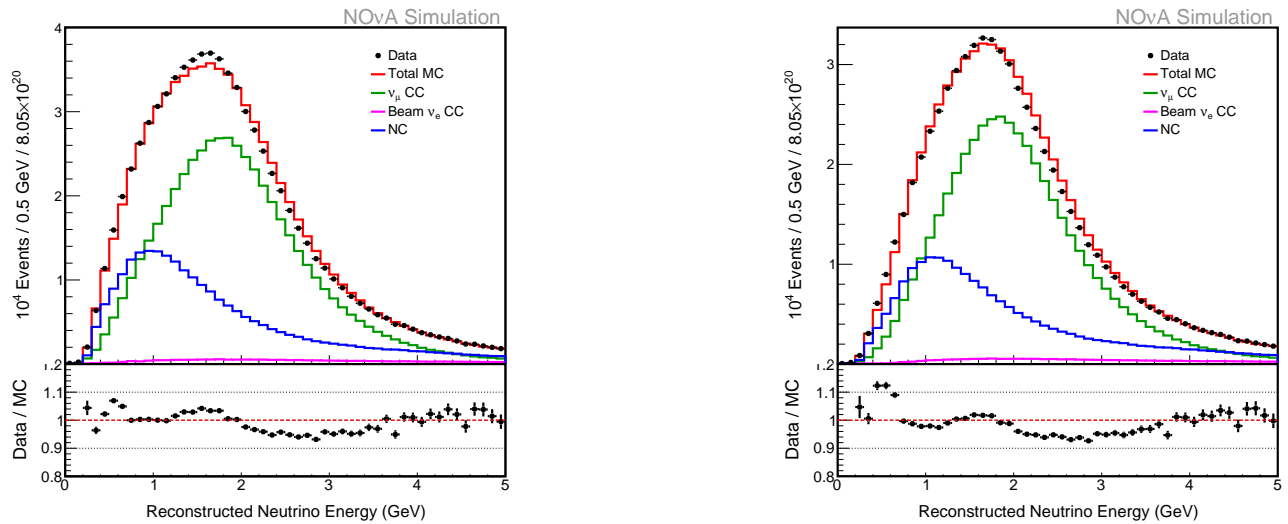

FIGURE 6.18: Shows the preselection distribution of reconstructed neutrino energy for the events which pass the $\nu_{e}$ prong length (left) and Ptp (right) selection. The MC is normalized to the data POT of $8.05 \mathrm{e}+20$.
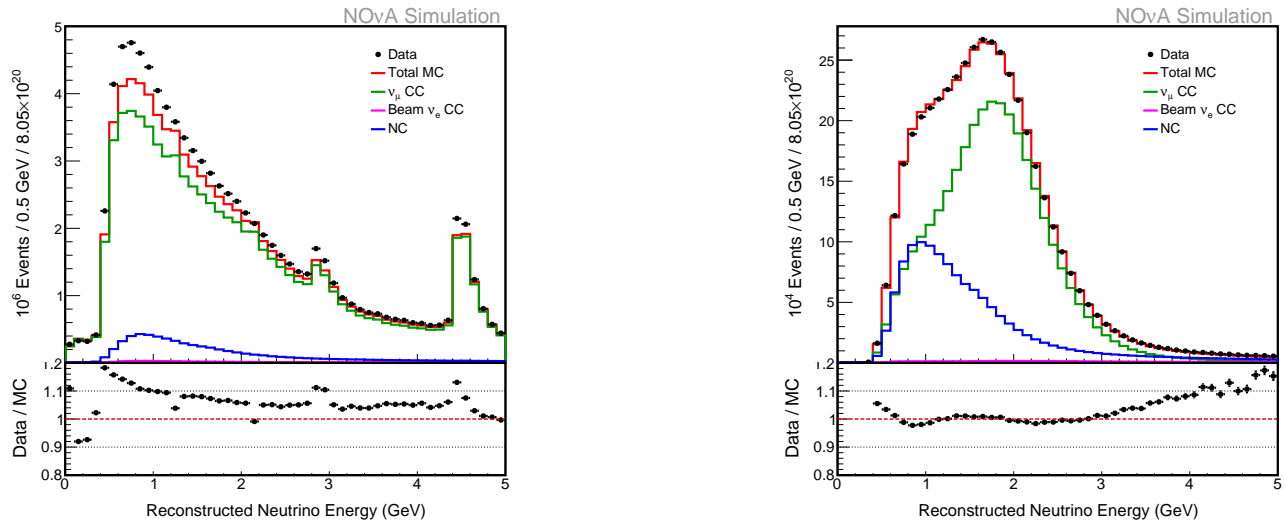

FiguRE 6.19: Shows the preselection distribution of reconstructed neutrino energy for the events which pass the $\nu_{\mu}$ quality (left) and containment (right) selection. The MC is normalized to the data POT of $8.05 \mathrm{e}+20$.

the data driven $\nu_{e}$-group ND corrections were applied. For the $\nu_{\mu}$ selected samples a $1.6 \%$ difference is seen which is consistent with the data-MC difference observed by the $\nu_{\mu}$ group for the 2017 analysis (see NOvA-doc-db 23640).

The following datasets and weights were used for this study

\section{- GENIE}

prod_caf_R17-03-01-prod3reco.d_nd_genie_nonswap_fhc_nova_v08_full_v1

- Data

prod_caf_R17-03-01-prod3reco.d_nd_numi_fhc_full_v1_goodruns

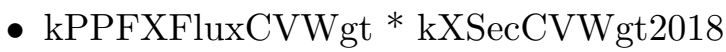

- Normalized to data of $8.089877 \mathrm{e}+20$ POT 
We can not apply data-driven corrections such as the $\nu_{e}$ group does, as it is very hard to decouple those data-MC difference as not being due to any potential sterile signal. But the data-MC differences seen are covered by our uncertainties.

\subsubsection{1 $\nu_{e}$ Unblinded Variables}
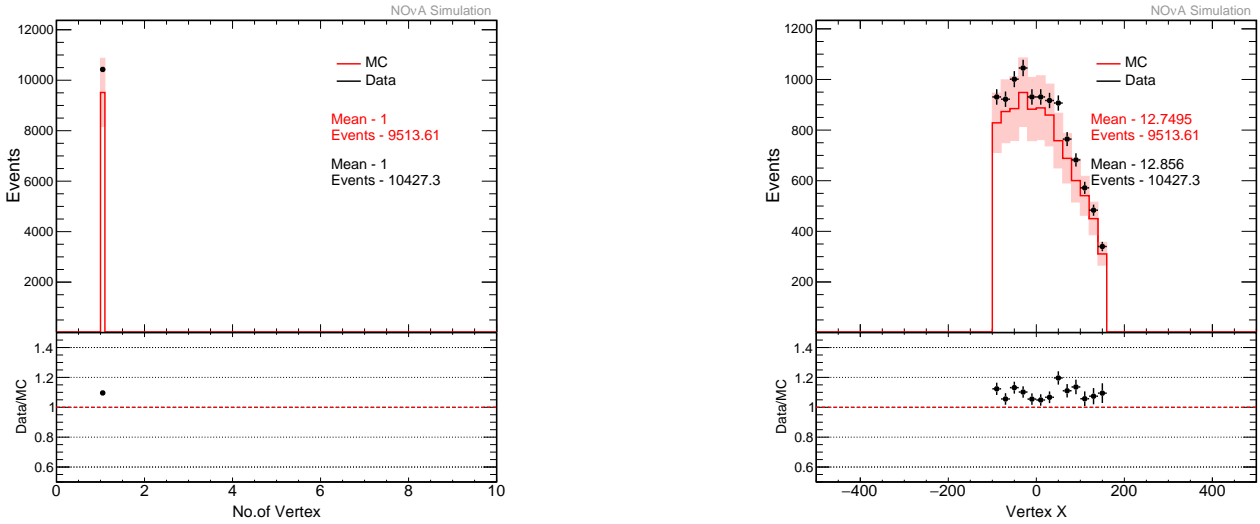

Figure 6.20: Shows the distribution of the number of vertices (left) and vertex $\mathrm{X}$ (right) with the $\nu_{e}$ selection applied.
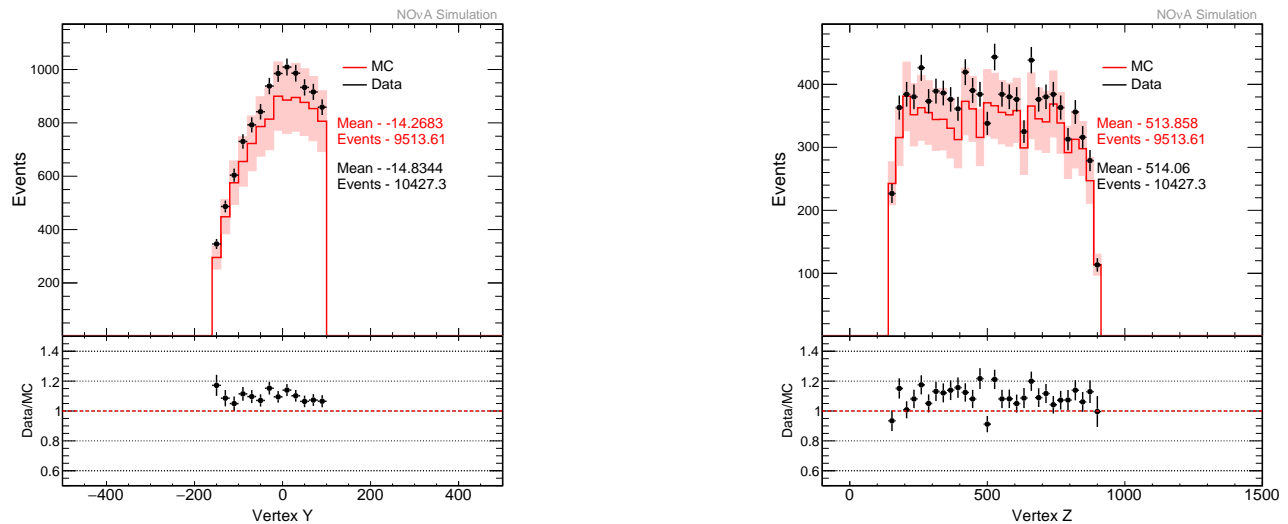

Figure 6.21: Shows the distribution of the vertex Y (left) and vertex Z (right) with the $\nu_{e}$ selection applied.

\subsubsection{2 $\nu_{\mu}$ Unblinded Variables}

\subsubsection{Muon Removed Electron Events}

An additional cross-check we can do is to look at MRE events. Muon Removed Electron (added) events are a handle to check the reconstruction efficiency of electrons in data and MC. In data and MC, $\nu_{\mu}$ events are selected, and the hits corresponding to the muon are removed, and a simulated electron of matching energy and direction is added in its place. A detailed description of MRE's is found in [144]. The distribution of CVNe and the 

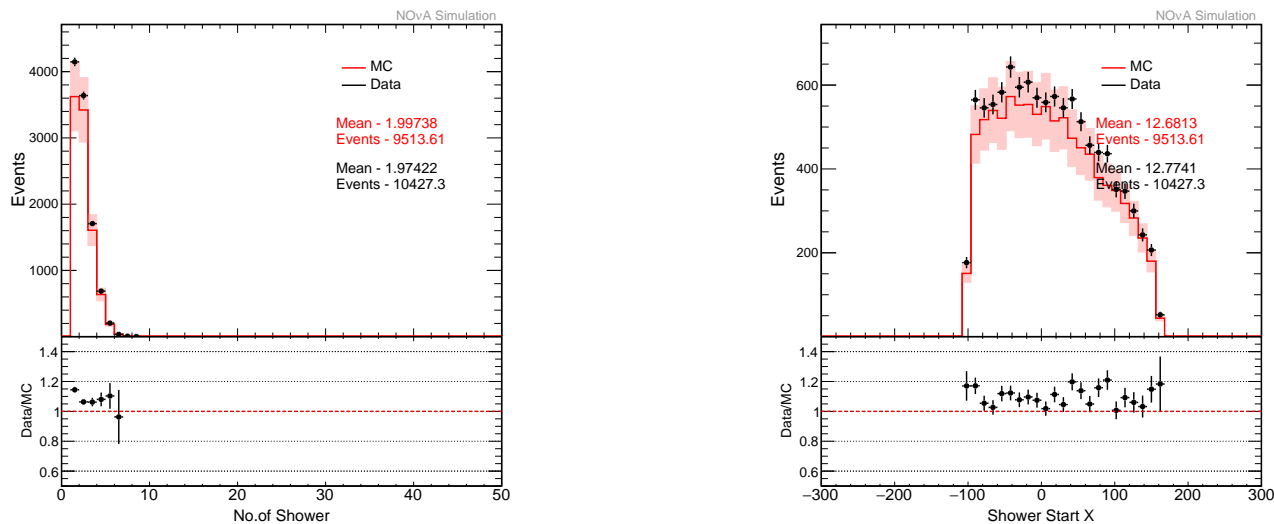

FiguRE 6.22: Shows the distribution of the number of showers (left) and shower start $\mathrm{X}$ (right) with the $\nu_{e}$ selection applied.
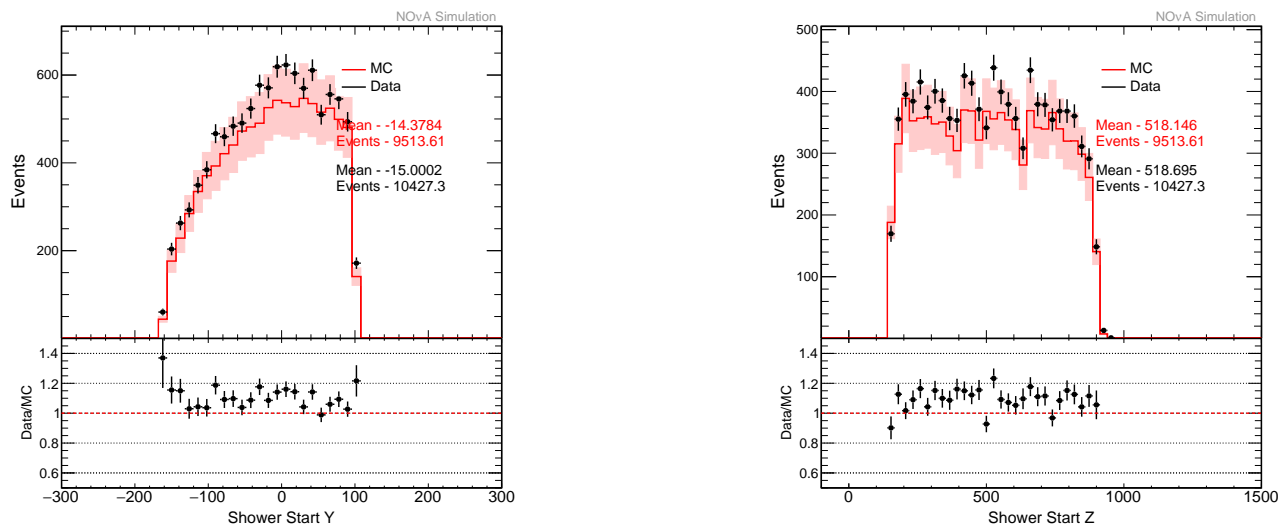

FIGURE 6.23: Shows the distribution of the shower start Y (left) and shower start Z (right) with the $\nu_{e}$ selection applied.

reconstructed energy distribution of $\nu_{e}$ selected sample for both data and MC is shown in Fig. 6.31. In Fig. 6.32, we show the distribution of reconstructed of MRE events just after the preselection and full $\nu_{e}$ selection with CVNe $>0.95$. We have used the following SAM definitions, with selection cut kMRParentSliceCut and kNueNDSecondAnaPresel and the standards weights, kPPFXFluxCVWgt and kXSecCVWgt2018.

It can be seen for this sample that the MC is slightly biased to being more electron like and that the energy is shifted in data to be lower. The overall efficiency is seen to be very similar between data and $\mathrm{MC}$ at around the $2 \%$ for the $0.95 \mathrm{CVNe}$ cut. The data-MC mean energy is also seen to differ by about $2 \%$, as is the calorimetric energy and number of hits. The full reason for these differences is not fully understood. We do not apply any additional corrections from this cross check as the cause of them is not fully understood. But the differences seen are covered by the systematic uncertainties.

\section{- GENIE}

prod_mrecaf_R17-03-01-prod3reco.k_nd_genie_nonswap_fhc_nova_v08_full_v1 

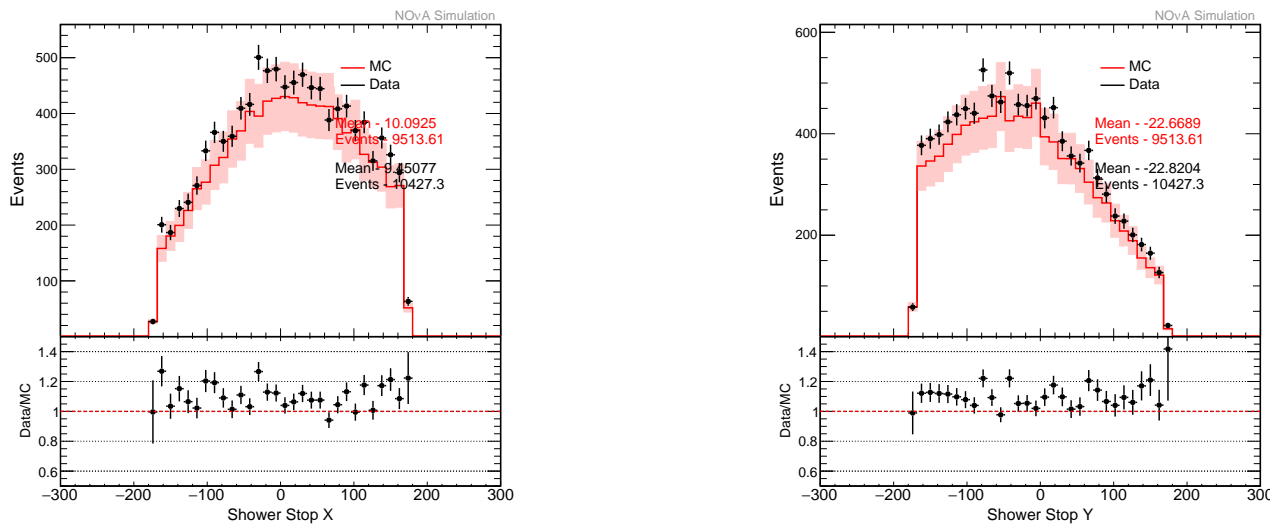

FiguRE 6.24: Shows the distribution of the shower stop X (left) and shower stop Y (right) with the $\nu_{e}$ selection applied.

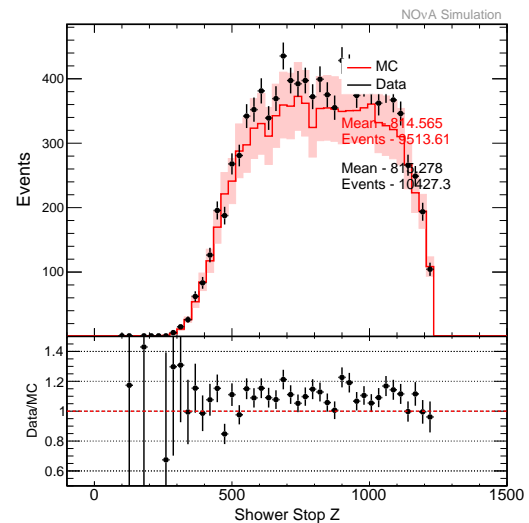

FIgURE 6.25: Shows the distribution of the shower stop Z (left) with the $\nu_{e}$ selection applied.

- Data

prod_mrecaf_R17-03-01-prod3reco.k_nd_numi_fhc_full_v1_goodruns 

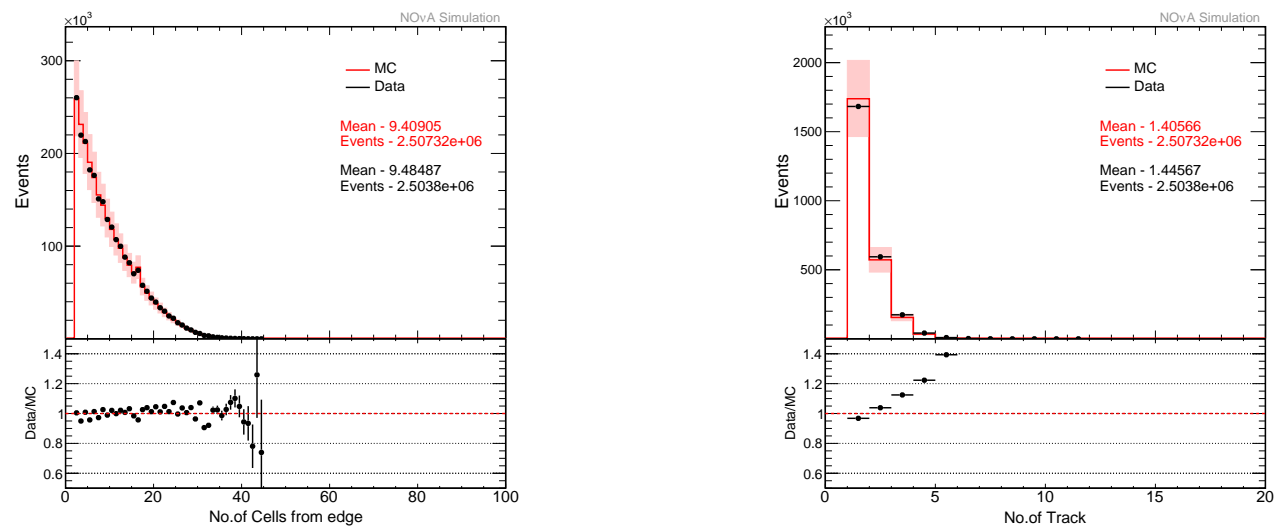

Figure 6.26: Shows the distribution of the number of cells from edge (left) and the number of tracks (right) with the $\nu_{\mu}$ selection applied.
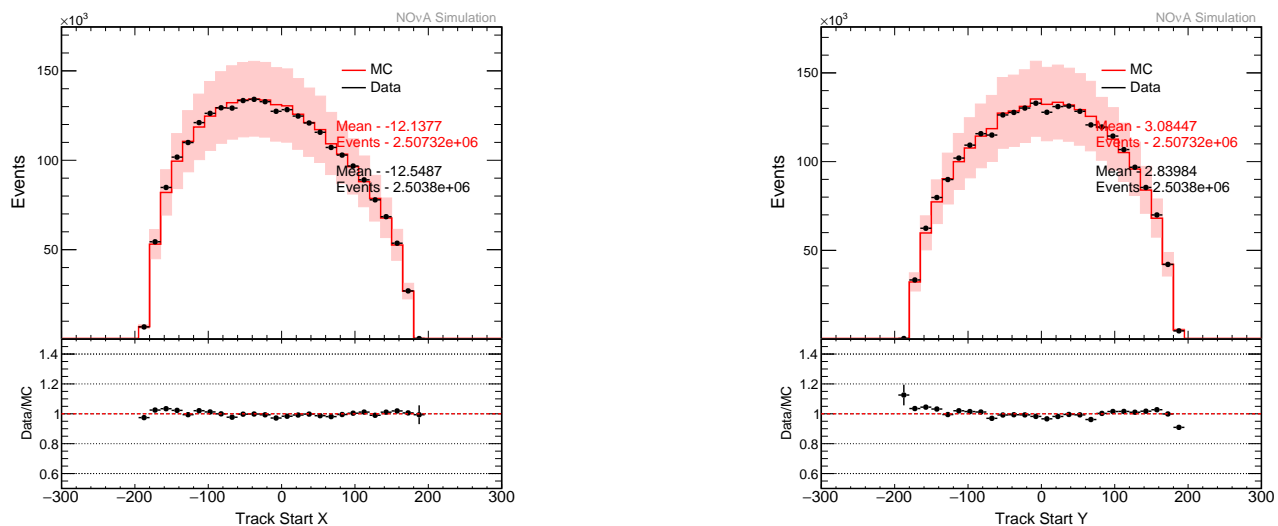

FIGURE 6.27: Shows the distribution of track start X (left) and track start Y (right) with the $\nu_{\mu}$ selection applied..
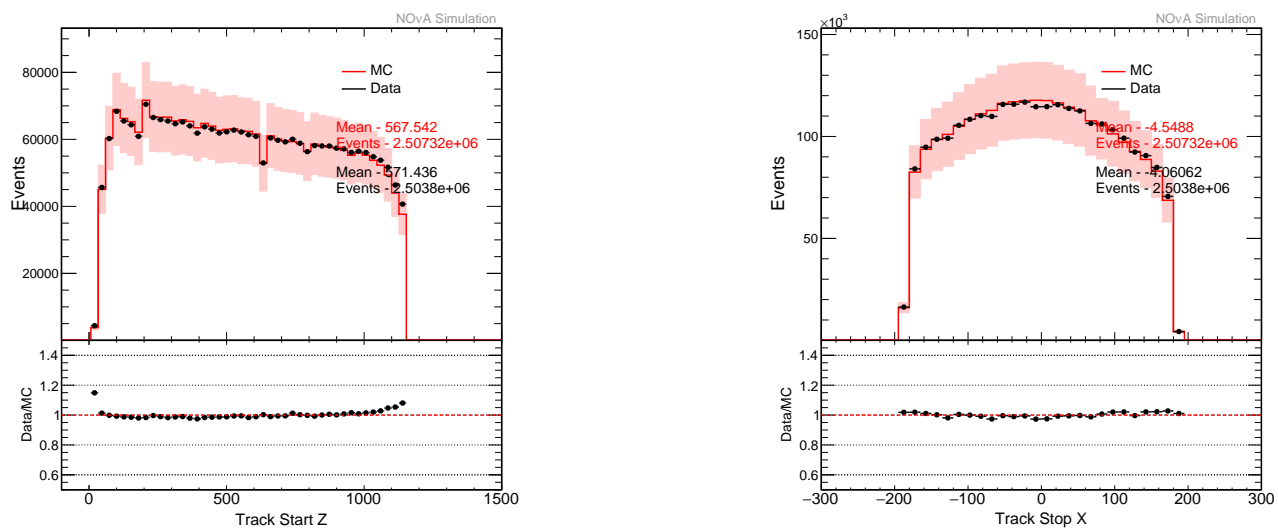

FIGURE 6.28: Shows the distribution of track start Z (left) and track stop X (right) with the $\nu_{\mu}$ selection applied.. 

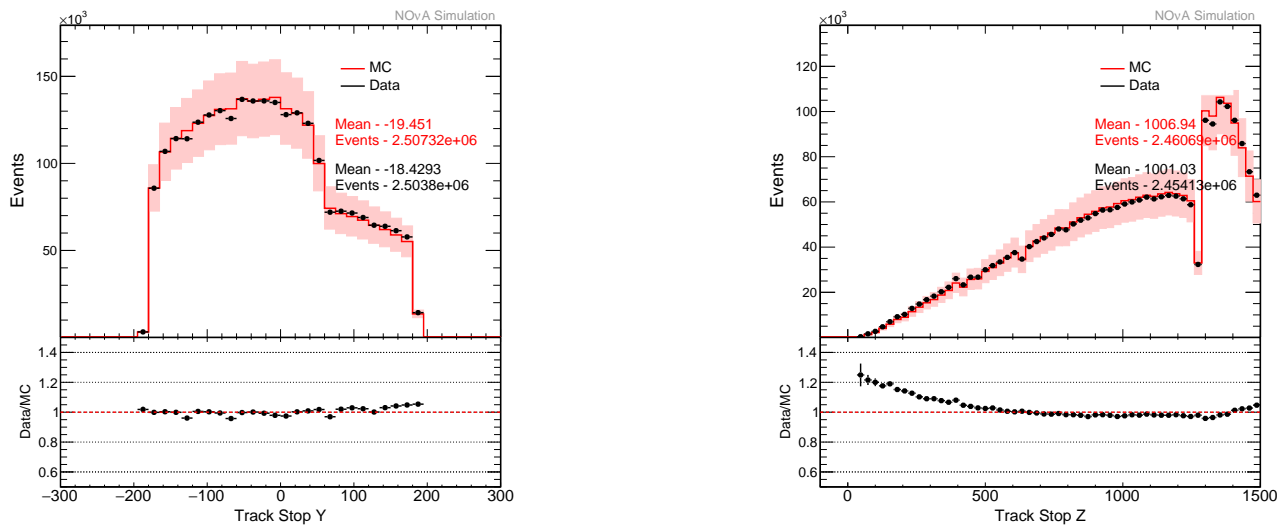

Figure 6.29: Shows the distribution of track stop Y (left) and track stop Z (right) with the $\nu_{\mu}$ selection applied..

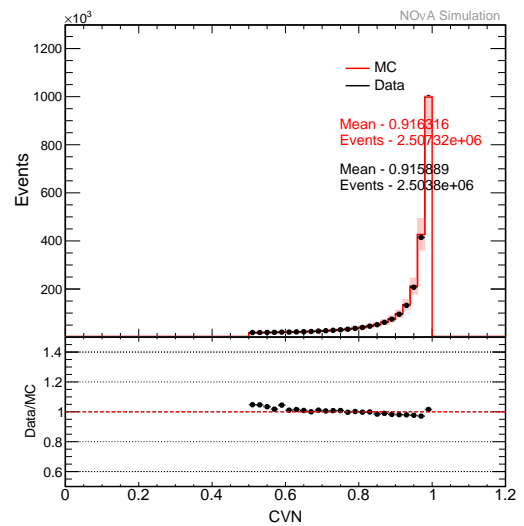

FiguRE 6.30: Shows the distribution of CVN for the $\nu_{\mu}$ selected events after the CVNm $>0.5$.
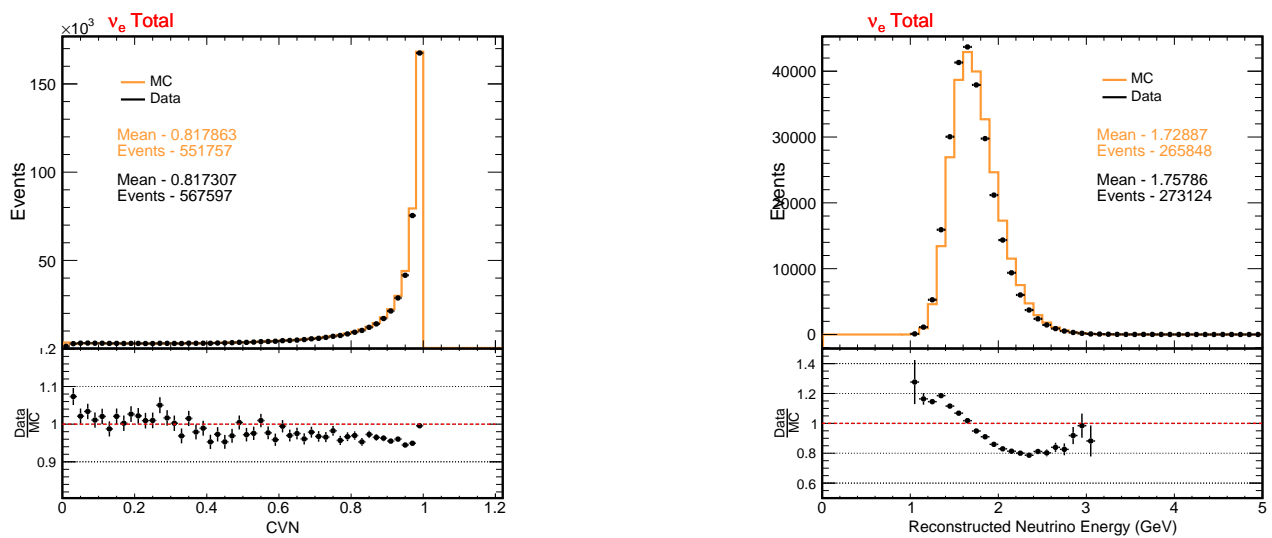

Figure 6.31: Shows the distribution of CVNe for the $\nu_{e}$ preselected sample (left) and the distribution of reconstructed neutrino energy for the $\nu_{e}$ selected sample with CVNe $>0.95$ (right). $\mathrm{MC}$ is normalized to the data POT of $8.05 \mathrm{e}+20$. 

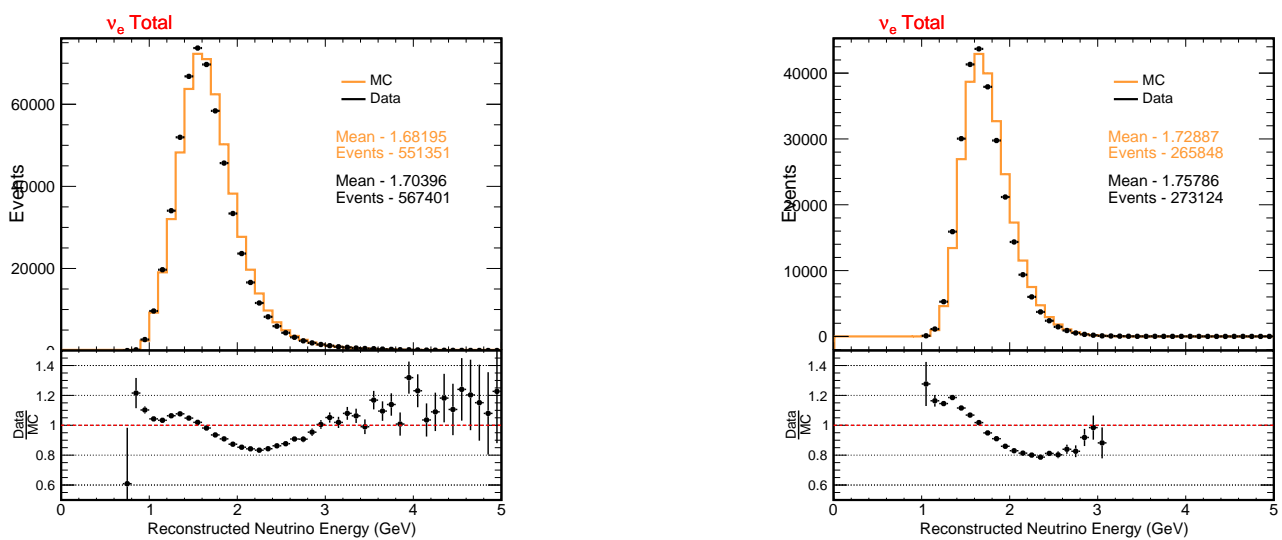

Figure 6.32: Shows the distribution of reconstructed neutrino energy for the $\nu_{e}$ preselected sample (left) and the fully selected sample with CVNe $>0.95$ (right). MC is normalized to the data POT of $8.05 \mathrm{e}+20$. 



\section{Chapter 7}

\section{Conclusion and Future}

In this thesis, I have shown the NOvA experiment's potential to probe the anomalous results seen by the past short-baseline experiments by searching for the $\nu_{e}$ appearance and $\nu_{\mu}$ disappearance at the NOvA Near Detector. If those anomalous results were due to the existence of fourth flavour neutrino with a mass larger than the standard 3-flavour neutrinos, the NOvA Near-Detector is expected to see the modifications in the NuMI flux due to active to sterile oscillations.

As the NOvA experiment is designed to study the active neutrino oscillations, the experiments' entire analysis framework, the CAFAna framework, is designed and tuned for that purpose. To perform this analysis at the Near Detector, it was necessary for the addition of new tools to the current CAFAna framework. I have developed these required analysis tools for my thesis analysis.

For the active oscillation analysis which uses data from both the Near and Far Detectors, there is a cancellation of correlated systematics such as the detector, flux related systematics. For the analyses which use only the Near Detector, there is not such cancellation of systematic uncertainties, but that's not the end of the story. For this analysis, we performed a joint analysis between the $\nu_{e}$ appearance and $\nu_{\mu}$ disappearance, having these two channels enable us to also achieve the cancellation of any correlated systematic uncertainties. I have demonstrated the advantage of performing the joint analysis rather using the single neutrino oscillation channel in this thesis. I have shown the NOvA's potential in probing the existence of the sterile neutrinos with the currently available NOvA's collected data, in terms of the $(3+1)$ neutrino mixing parameters. The results show that NOvA can set the strong limits on mixing angles, for example, NOvA's limits on $\theta_{24}$ at $\Delta \mathrm{m}_{41}^{2}>1 \mathrm{eV}^{2}$ are stronger than the predecessor sterile neutrino experiments. NOvA's sensitivities when including all systematic uncertainties for the mixing angle $\theta_{\mu e}$ can exclude the $90 \%$ C.L. results of LSND experiment. 
We performed the mock data tests to test the robustness of framework to test whether we are in a situation to correctly interpret the results with the real data. We confirm that the framework is performing as expected.

We have performed cross-checks of the data and MC efficiency, and of the selected events while keeping ourselves blind to the possible signal of sterile neutrinos.

Currently, the studies conducted so far are well understood, but some cross checks continue before we can look at the data and produce the final NOvA's results from this analysis. This thesis contains all the results except the final data results. We plan to use the data collected between October 2014 and May 2017 which corresponds to $8.05 \mathrm{e}+20$ Protons On Target. More the data, better the results. There is also a lot of room for improvements for this analysis. By constraining the hadron production uncertainties, improving the cross-section models, with the use of Near Detector specific energy estimators, this analysis outputs the most competitive results on $(3+1)$ mixing parameter limits. Also, the same study can be done for NOvA's anti-neutrino data.

We expect to publish the results for the coming Neutrino 2018 conference. Currently, we are planning to publish the results from this analysis in the Physical Review Letters in summer 2018. 


\section{Appendix A}

\section{Samples Used}

\section{A.1 Systematic Samples}

Here is the list of production 3 systematically shifted SAM definitions as used.

- Nominal

- prod_caf_R17-03-01-prod3reco.d_nd_genie_nonswap_fhc_nova_v08_full_v1

- Calibration Positive Offset

- prod_decaf_R17-03-01-prod3reco.h_nd_genie_nonswap_fhc_nova_v08_periods1235_calibshift-nd-xyview-neg-offset_nue_or_numu_or_nus_contain_v1

- Calibration Negtive Offset

- prod_decaf_R17-03-01-prod3reco.h_nd_genie_nonswap_fhc_nova_v08_periods1235_calibshift-nd-xyview-pos-offset_nue_or_numu_or_nus_contain_v1

- Calibration shape

- prod_decaf_R17-03-01-prod3reco.j_nd_genie_nonswap_fhc_nova_v08_full_calib-shiftnd-func_nue_or_numu_or_nus_contain_v1

- Light up and Calib down

- prod_decaf_R17-03-01-prod3reco.h_nd_genie_nonswap_fhc_nova_v08_full_lightmodellightup-calibdown_nue_or_numu_or_nus_contain_v1

- Light down and Calib up 
- prod_decaf_R17-03-01-prod3reco.h_nd_genie_nonswap_fhc_nova_v08_full_lightmodellightdown-calibup_nue_or_numu_or_nus_contain_v1

- Cherenkov Variation

- prod_decaf_R17-03-01-prod3reco.h_nd_genie_nonswap_fhc_nova_v08_full_ckv-protonshift-down_nue_or_numu_or_nus_contain_v1

- Nominal (8149 files)

- prod_caf_R17-03-01-prod3reco.h_nd_genie_nonswap_fhc_nova_v08_full_nuecc-overlay_v1

- Calibration Positive Offset (6545 files)

- prod_caf_R17-03-01-prod3reco.k_nd_genie_nonswap_fhc_nova_v08_full_calib-shiftnd-xyview-pos-offset_nuecc-overlay_v1

- Calibration Negative Offset (7671 files)

- prod_caf_R17-03-01-prod3reco.k_nd_genie_nonswap_fhc_nova_v08_full_calib-shiftnd-xyview-neg-offset_nuecc-overlay_v1

- Calibration shape (8041 files)

- prod_caf_R17-03-01-prod3reco.k_nd_genie_nonswap_fhc_nova_v08_full_calib-shiftnd-func_nuecc-overlay_v1

- Light up and Calib down (4529 files)

- prod_caf_R17-03-01-prod3reco.k_nd_genie_nonswap_fhc_nova_v08_full_lightmodellightup-calibdown_nuecc-overlay_v1

- Light down and Calib up (4199 files)

- prod_caf_R17-03-01-prod3reco.k_nd_genie_nonswap_fhc_nova_v08_full_lightmodellightdown-calibup_nuecc-overlay_v1

- Cherenkov Variation (5248 files)

- prod_caf_R17-03-01-prod3reco.k_nd_genie_nonswap_fhc_nova_v08_full_ckv-protonshift-down_nuecc-overlay_v1 


\section{Appendix B}

\section{New Tools Developed For SBL Analysis}

\section{B.1 CAFAna Framework}

CAFAna is a collection of classes to perform any oscillation analysis in the NOvA experiment [126]. CAFAna uses Common Analysis Format (CAF) files as input. CAF files are lightweight and incorporate all the necessary information needed for the main analyses.

The current CAFAna framework needed to be upgraded to include the necessary tools used to perform any SBL sterile analyses. In general, the neutrino oscillation probabilities are expressed as a function of $L / E$, where $L$ is the simulated distance traveled by a neutrino before detection and $\mathrm{E}$ is the simulated energy of a neutrino. The detectors sited close (in the order of a few meters) to the neutrino source observe neutrinos traveling varying distances (like a line source) whereas the detectors placed far (in the order of few kilometers) from the neutrino source observe neutrinos coming from one source (like a point source). Thus, in Near Detector specific analyses, especially any oscillation analysis, the oscillation probabilities should consider the variation in the baseline length L. The current CAFAna framework is developed based on the oscillations at the Far Detector, so probabilities are expressed as a function of energy E at a fixed baseline length L. All of these new tools exist in a special feature branch, T17-10-10-sblana.a that will be merged into trunk in due course. 


\section{B.1.1 NDOscCurve}

NDOscCurve is a CAFAna class that provides the oscillation probability binned into a histogram as a function of the ratio between the simulated distance traveled by a neutrino before it is detected and simulated energy of the neutrino $(\mathrm{L} / \mathrm{E})$. The probability is calculated for the 3 -flavor oscillations or oscillations involving sterile neutrinos. It is similar in design and functionality with the OscCurve class in the standard CAFAna framework, where the oscillation probability is binned as a function of simulated energy $\mathrm{E}$ at a fixed $810 \mathrm{~km}$ baseline.

\section{B.1.2 NDOscillatableSpectrum}

The NDOscillatableSpectrum class provides support for creating CAFAna spectra with true $\mathrm{L} / \mathrm{E}$ information. It functions similar in fashion to providing a two-dimensional ROOT histogram, with the horizontal $\mathrm{X}$-axis representing any reconstructed variable and the vertical $\mathrm{Y}$-axis representing the $\mathrm{L} / \mathrm{E}$ ratio. It functions similar in design to the standard OscillatableSpectrum class that is used for 3-flavor oscillation analyses at the Far Detector where the Y-axis represents simply the simulated neutrino energy due to the fixed baseline assumption. This class is extremely useful in the SBL oscillation analysis to get the event rates after neutrino oscillations are considered. In the analysis, the class object (ultimately a 2D histogram) is filled with events which pass either $\nu_{e}, \nu_{\mu}$, or any customized selection which includes both simulated signal and background. In order to apply oscillations, the bin content of each bin along the Y-axis of an NDOscillatableSpectrum is weighted with the corresponding oscillation probability in that bin. This object can be made for any component with any systematic shift applied.

\section{B.1.3 NDExtrap}

In Far Detector analyses, one extrapolates the simulated Near Detector event rates to the Far Detector for a predicted signal with oscillation assumptions considered [145]. This is a Near Detector-only analysis so there is no actual extrapolation involved in the analysis. The NDExtrap class provides an interface that allows for design consistency with the current CAFAna framework. Rather than extrapolating components, this class takes ownership of NDOscillatableSpectrum objects for each selected component so that they can be used in a prediction class to apply oscillations. 


\section{B.1.4 NDPredictionExtrap}

NDPredictionExtrap is a class for obtaining the predicted event rates after oscillations applied to every selected component. This object can return the total predicted event rate or individual predicted rate for each component. Examples include beam components $\nu_{e} \mathrm{CC}, \nu_{\mu} \mathrm{CC}$, Neutral Current etc. oscillated with $\nu_{e} \rightarrow \nu_{e}, \nu_{\mu} \rightarrow \nu_{\mu}$, NC oscillations respectively.

\section{B.1.5 PredictionInterpJointAna}

PredictionInterpJointAna is the key class for this analysis. It has very rich functionality as it does multiple things at a time. This object holds the systematically shifted prediction objects explained in [B.1.4] for every type of systematics. It can return the predicted event rate for any systematic shift other than $( \pm 3 \sigma, \pm 2 \sigma, \pm 1 \sigma)$ using the concept called "Cubic Interpolation" [127]. This is slightly different from what we have for 3-flavor oscillation analysis.

This analysis uses joint-fit method which means that we fit for $\nu_{\mu}$ disappearance and $\nu_{e}$ appearance simultaneously. Since $\nu_{e}$ and $\nu_{\mu}$ have separate systematics, we need to be able to apply systematics separately. We make the prediction objects for both $\nu_{e}$ and $\nu_{\mu}$ systematics, but we set shift to 0 for $\nu_{\mu}$ systematics in the $\nu_{e}$ prediction and vice versa. This way we handle the correlated and uncorrelated systematics correctly. 

Appendix C

GENIE Systematic Tables

C.1 GENIE Systematic Table For $\nu_{e}$ Selected Sample 


\begin{tabular}{|c|c|c|c|c|c|c|c|c|c|c|}
\hline \multirow{2}{*}{ Syst.Parameter } & \multicolumn{2}{|c|}{$\nu_{e}$ sig } & \multicolumn{2}{|c|}{ Total bkg } & \multicolumn{2}{|c|}{$\nu_{\mu}$} & \multicolumn{2}{|c|}{ beam $\nu_{e}$} & \multicolumn{2}{|c|}{ NC } \\
\hline & $+1 \sigma(\%)$ & $-1 \sigma(\%)$ & $+1 \sigma(\%)$ & $-1 \sigma(\%)$ & $+1 \sigma(\%)$ & $-1 \sigma(\%)$ & $+1 \sigma(\%)$ & $-1 \sigma(\%)$ & $+1 \sigma(\%)$ & $-1 \sigma(\%)$ \\
\hline MaCCQE_reduced & 1.6 & 1.7 & 1.2 & 1.2 & 0.097 & 0.086 & 1.6 & 1.7 & 0 & 0 \\
\hline RPAShapeenh2017 & 5.5 & 4.6 & 4.5 & 3.6 & 3.2 & 2.2 & 5.5 & 4.5 & 0 & 0 \\
\hline RPAShapesupp2017 & 1.9 & 1.9 & 1.4 & 1.4 & 0.2 & 0.2 & 1.9 & 1.9 & 0 & 0 \\
\hline RPAShapeRES & 4.2 & 0 & 2.8 & 0 & 0.5 & 0 & 3.9 & 0 & 0 & 0 \\
\hline MECq0Shape & 9.4 & 7.2 & 6.3 & 5.7 & 0.12 & 0.073 & 8.5 & 7.8 & 0.24 & 0.24 \\
\hline MECInitStateNPFrac & 1.4 & 1.4 & 1 & 1 & 0.036 & 0.036 & 1.3 & 1.3 & 0.03 & 0.03 \\
\hline MECEnuShape & 4.1 & 4.1 & 2.7 & 2.7 & 0.092 & 0.092 & 3.6 & 3.6 & 0.0069 & 0.0069 \\
\hline DISvpCC0pi & 0.36 & 0.36 & 0.51 & 0.51 & 1.7 & 1.7 & 0.4 & 0.4 & 0 & 0 \\
\hline DISvpCC1pi & 0.2 & 0.2 & 0.22 & 0.22 & 0.33 & 0.33 & 0.24 & 0.24 & 0 & 0 \\
\hline DISvpCC2pi & 0.63 & 0.63 & 1.2 & 1.2 & 5 & 5 & 0.74 & 0.74 & 0 & 0 \\
\hline DISvpCC3pi & 0.2 & 0.2 & 0.33 & 0.33 & 0.9 & 0.9 & 0.28 & 0.28 & 0 & 0 \\
\hline DISvpNCOpi & 0 & 0 & 0.43 & 0.43 & 0 & 0 & 0 & 0 & 3.3 & 3.3 \\
\hline DISvpNC1pi & 0 & 0 & 0.57 & 0.57 & 0 & 0 & 0 & 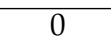 & 4.3 & 4.3 \\
\hline DISvpNC2pi & 0 & 0 & 0.87 & 0.87 & 0 & 0 & 0 & 0 & 6.5 & 6.5 \\
\hline DISvpNC3pi & 0 & 0 & 0.22 & 0.22 & 0 & 0 & 0 & 0 & 1.6 & 1.6 \\
\hline DISvnCC0pi & 0.019 & 0.019 & 0.03 & 0.03 & 0.045 & 0.045 & 0.032 & 0.032 & 0 & 0 \\
\hline DISvnCC & 0.95 & 0.95 & 3 & 3 & 16 & 16 & 1.2 & 1.2 & 0 & 0 \\
\hline DISvnCC2pi & 0.33 & 0.33 & 1.5 & 1.5 & 8.3 & 8.3 & 0.51 & 0.51 & 0 & 0 \\
\hline DISvnCC3pi & 0.058 & 0.058 & 0.2 & 0.2 & 0.91 & 0.91 & 0.11 & 0.11 & 0 & 0 \\
\hline DISvi & 0 & 0 & 0.0059 & 0.0059 & 0 & 0 & 0 & 0 & 0.044 & 0.044 \\
\hline DISvnNC1pi & 0 & 0 & 0.98 & 0.98 & 0 & 0 & 0 & 0 & 7.3 & 7.3 \\
\hline DISvnNC2pi & 0 & 0 & 1 & 1 & 0 & 0 & 0 & 0 & 7.7 & 7.7 \\
\hline DISvnNC3pi & 0 & 0 & 0.23 & 0.23 & 0 & 0 & 0 & 0 & 1.7 & 1.7 \\
\hline DISvbarpCC0pi & 0.017 & 0.017 & 0.032 & 0.032 & 0.018 & 0.018 & 0.04 & 0.04 & 0 & 0 \\
\hline DISvb & 0.042 & 0.042 & 0.12 & 0.12 & 0.026 & 0.026 & 0.16 & 0.16 & 0 & 0 \\
\hline DISvba & 0.01 & 0.01 & 0.029 & 0.029 & 0.036 & 0.036 & 0.033 & 0.033 & 0 & 0 \\
\hline DISvbarp & 0.0021 & 0.0021 & 0.0019 & 0.0019 & 0 & 0 & 0.0026 & 0.0026 & 0 & 0 \\
\hline DISvba & 0 & 0 & 0.031 & 0.031 & 0 & 0 & 0 & 0 & 0.23 & 0.23 \\
\hline arpNC1pi & 0 & 0 & 0.055 & 0.055 & 0 & 0 & 0 & 0 & 0.41 & 0.41 \\
\hline DISvb & 0 & 0 & 0.046 & 0.046 & 0 & 0 & 0 & 0 & 0.35 & 0.35 \\
\hline DISvb & 0 & 0 & 0.008 & 0.008 & 0 & 0 & 0 & 0 & 0.06 & 0.06 \\
\hline DISvb & 0 & 0 & 0 & 0 & 0 & 0 & 0 & 0 & 0 & 0 \\
\hline DISvba & 0.0096 & 0.0096 & 0.02 & 0.02 & 0.0076 & 0.0076 & 0.026 & 0.026 & 0 & 0 \\
\hline DISvl & 0.01 & 0.01 & 0.051 & 0.051 & 0.019 & 0.019 & 0.066 & 0.066 & 0 & 0 \\
\hline DISvba & 0.0038 & 0.0038 & 0.011 & 0.011 & 0.0012 & 0.0012 & 0.015 & 0.015 & 0 & 0 \\
\hline DISvbarnNC0pi & 0 & 0 & 0 & 0 & 0 & 0 & 0 & 0 & 0 & 0 \\
\hline DISvba & 0 & 0 & 0.021 & 0.021 & 0 & 0 & 0 & 0 & 0.16 & 0.16 \\
\hline DISvb & 0 & 0 & 0.053 & 0.053 & 0 & 0 & 0 & 0 & 0.39 & 0.39 \\
\hline DISvbarnN & 0 & 0 & 0.013 & 0.013 & 0 & 0 & 0 & 0 & 0.096 & 0.096 \\
\hline radcorrn & 2 & 2 & 1.4 & 1.4 & 0 & 0 & 1.9 & 1.9 & 0 & 0 \\
\hline radcorrnuebar & 0.042 & 0.042 & 0.098 & 0.098 & 0 & 0 & 0.13 & 0.13 & 0 & 0 \\
\hline & 1.9 & 1.9 & 1.3 & 1.3 & 0 & 0 & 1.7 & 1.7 & 0 & 0 \\
\hline MaNCEL & 0 & 0 & 0.03 & 0.013 & 0 & 0 & 0 & 0 & 0.22 & 0.095 \\
\hline EtaNCEL & 0 & 0 & 0.00035 & 0.00033 & 0 & 0 & 0 & 0 & 0.0026 & 0.0025 \\
\hline VecFFCCQEshape & 0.41 & 0.41 & 0.26 & 0.26 & 0.0036 & 0.0035 & 0.35 & 0.35 & 0 & 0 \\
\hline MaCCRES & 6.5 & 7.1 & 6.1 & 6.1 & 7.1 & 6.2 & 7 & 7.2 & 0 & 0 \\
\hline & 3.6 & 3.4 & 3.3 & 3 & 4 & 3.5 & 3.8 & 3.4 & 0 & 0 \\
\hline MaNCRES & 0 & 0 & 1.2 & 0.92 & 0 & 0 & 0 & 0 & 9.2 & 6.9 \\
\hline MvNCRES & 0 & 0 & 0.29 & 0.24 & 0 & 0 & 0 & 0 & 2.2 & 1.8 \\
\hline & 0.81 & 0.85 & 2.3 & 2.4 & 0.081 & 0.081 & 0.83 & 0.85 & 13 & 13 \\
\hline R0COHpi & 0.84 & 0.81 & 2.4 & 2.3 & 0.081 & 0.081 & 0.84 & 0.82 & 13 & 13 \\
\hline
\end{tabular}

TABLE C.1: Shows the $\pm 1 \sigma \%$ shifts for all the GENIE systematic parameters for the $\nu_{e}$ selected sample. Continued in the next page. 


\begin{tabular}{|c|c|c|c|c|c|c|c|c|c|c|}
\hline \multirow{2}{*}{ Syst.Parameter } & \multicolumn{2}{|c|}{$\nu_{e} \operatorname{sig}$} & \multicolumn{2}{|c|}{ Total bkg } & \multicolumn{2}{|c|}{$\nu_{\mu}$} & \multicolumn{2}{|c|}{ beam $\nu_{e}$} & \multicolumn{2}{|c|}{$\mathrm{NC}$} \\
\hline & $+1 \sigma(\%)$ & $-1 \sigma(\%)$ & $+1 \sigma(\%)$ & $-1 \sigma(\%)$ & $+1 \sigma(\%)$ & $-1 \sigma(\%)$ & $+1 \sigma(\%)$ & $-1 \sigma(\%)$ & $+1 \sigma(\%)$ & $-1 \sigma(\%)$ \\
\hline AhtBY & 0.0093 & 0.0096 & 0.2 & 0.21 & 0.65 & 0.66 & 0.035 & 0.036 & 0.68 & 0.7 \\
\hline BhtBY & 0.015 & 0.015 & 0.32 & 0.33 & 1 & 1.1 & 0.051 & 0.053 & 1.1 & 1.1 \\
\hline CV1uBY & 0.0069 & 0.0077 & 0.19 & 0.21 & 0.65 & 0.72 & 0.023 & 0.026 & 0.66 & 0.73 \\
\hline CV2uBY & 0.0067 & 0.0067 & 0.18 & 0.18 & 0.63 & 0.63 & 0.022 & 0.022 & 0.64 & 0.64 \\
\hline NormDISCC & 0 & 0 & 0 & 0 & 0 & 0 & 0 & 0 & 0 & 0 \\
\hline RnubarnuCC & 0 & 0 & 0 & 0 & 0 & 0 & 0 & 0 & 0 & 0 \\
\hline DISNuclMod & 0 & 0 & 0 & 0 & 0 & 0 & 0 & 0 & 0 & 0 \\
\hline- & 0 & 0 & 0 & 0 & 0 & 0 & 0 & 0 & 0 & 0 \\
\hline AGKYxF1pi & 0.00078 & 0.0037 & 0.01 & 0.018 & 0.039 & 0.052 & 0.0011 & 0.00072 & 0.032 & 0.089 \\
\hline AGKYpT1pi & 0.0022 & 0.0015 & 0.00027 & 0.00085 & 0.0073 & 0.0098 & 0.00083 & 0.0011 & 0.0045 & 0.0095 \\
\hline FormZone & 0.26 & 0.37 & 1.8 & 3.4 & 6.7 & 13 & 0.41 & 0.55 & 4.7 & 10 \\
\hline MFP_pi & 0.76 & 0.67 & 1 & 1.1 & 2 & 2.5 & 0.73 & 0.68 & 1.8 & 2.2 \\
\hline MFP_N & 2.6 & 2.6 & 2 & 2.1 & 1.1 & 1.3 & 2.5 & 2.6 & 0.27 & 0.31 \\
\hline FrCEx_pi & 0.19 & 0.19 & 0.17 & 0.18 & 0.056 & 0.07 & 0.21 & 0.21 & 0.087 & 0.083 \\
\hline FrElas_pi & 0.048 & 0.048 & 0.046 & 0.045 & 0.24 & 0.24 & 0.0016 & 0.0023 & 0.12 & 0.12 \\
\hline FrInel_pi & 0.21 & 0.2 & 0.086 & 0.083 & 0.73 & 0.74 & 0.13 & 0.13 & 0.64 & 0.63 \\
\hline FrAbs_pi & 0.46 & 0.47 & 0.14 & 0.13 & 0.67 & 0.65 & 0.37 & 0.37 & 0.38 & 0.37 \\
\hline FrPiProd_pi & 0.0043 & 0.0042 & 0.039 & 0.039 & 0.17 & 0.17 & 0.0084 & 0.0087 & 0.18 & 0.18 \\
\hline FrCEx_N & 0.23 & 0.24 & 0.22 & 0.24 & 0.3 & 0.31 & 0.27 & 0.29 & 0.13 & 0.13 \\
\hline FrElas_N & 0.76 & 0.74 & 0.55 & 0.53 & 0.48 & 0.48 & 0.75 & 0.72 & 0.51 & 0.51 \\
\hline FrInel_N & 0.21 & 0.22 & 0.066 & 0.075 & 0.0091 & 0.013 & 0.049 & 0.061 & 0.24 & 0.24 \\
\hline FrAbs_N & 0.15 & 0.15 & 0.12 & 0.12 & 0.11 & 0.1 & 0.21 & 0.21 & 0.37 & 0.37 \\
\hline FrPiProd_N & 0.0082 & 0.0082 & 0.0028 & 0.0028 & 0.015 & 0.015 & 0.028 & 0.028 & 0.12 & 0.12 \\
\hline CCQEPauliSupViaKF & 1.7 & 1.4 & 1.3 & 1.1 & 0.041 & 0.02 & 1.7 & 1.5 & 0 & 0 \\
\hline CCQEMomDistroFGtoSF & 0 & 0 & 0 & 0 & 0 & 0 & 0 & 0 & 0 & 0 \\
\hline RDecBR1gamma & 0.0032 & 0.0032 & 0.025 & 0.025 & 0.06 & 0.06 & 0.0017 & 0.0017 & 0.14 & 0.14 \\
\hline RDecBR1eta & 0.27 & 0.27 & 0.24 & 0.24 & 2.4 & 2.4 & 0.33 & 0.33 & 1.2 & 1.2 \\
\hline Theta_Delta2Npi & 1.3 & 0.87 & 0.91 & 0.61 & 0.16 & 0.11 & 1.2 & 0.8 & 0.013 & 0.0086 \\
\hline
\end{tabular}

TABlE C.2: Shows the $\pm 1 \sigma \%$ shifts for all the GENIE systematic parameters for the $\nu_{e}$ selected sample. 
C.2 GENIE Systematic Table For $\nu_{\mu}$ Selected Sample 


\begin{tabular}{|c|c|c|c|c|c|c|c|c|c|c|}
\hline \multirow{2}{*}{ Syst.Parameter } & \multicolumn{2}{|c|}{ App. $\nu_{e}$} & \multicolumn{2}{|c|}{ Total bkg } & \multicolumn{2}{|c|}{\begin{tabular}{|l|}
$\nu_{\mu}$ \\
\end{tabular}} & \multicolumn{2}{|c|}{ beam $\nu_{e}$} & \multicolumn{2}{|c|}{$\mathrm{NC}$} \\
\hline & $+1 \sigma(\%)$ & & $+1 \sigma(\%)$ & & $+1 \sigma(\%)$ & $-1 \sigma(\%)$ & & $-1 \sigma(\%)$ & $+1 \sigma(\%)$ & $-1 \sigma(\%)$ \\
\hline MaCCQE_reduced & 0.58 & 0.43 & 0.33 & 0.25 & 0.86 & 0.9 & 0.45 & 0.35 & 0 & 0 \\
\hline RPAShapeenh2017 & 3.9 & 2.1 & 2.2 & 1.2 & 4.5 & 3.7 & 3.7 & 2.5 & 0 & 0 \\
\hline RPAShapesupp2017 & 0.13 & 0.13 & 0.074 & 0.074 & 1.4 & 1.4 & 0.11 & 0.11 & 0 & 0 \\
\hline RPAShapeRES & 0.0041 & 0 & 0.01 & 0 & 6.1 & 0 & 0.98 & 0 & 0 & 0 \\
\hline MECq0Shape & 0.081 & 0.078 & 0.035 & 0.16 & 4.6 & 0.38 & 0.059 & 0.85 & 0.026 & 0.29 \\
\hline MECInitStateNPFrac & 0.028 & 0.028 & 0.02 & 0.02 & 1.1 & 1.1 & 0.15 & 0.15 & 0.0077 & 0.0077 \\
\hline MECEnuShape & 0.25 & 0.25 & 0.15 & 0.15 & 3.5 & 3.5 & 0.36 & 0.36 & 0.0094 & 0.0094 \\
\hline DISvpCC0pi & 2.9 & 2.9 & 1.7 & 1.7 & 0.85 & 0.85 & 2.8 & 2.8 & 0 & 0 \\
\hline DISvpCC1pi & 2.3 & 2.3 & 1.3 & 1.3 & 0.47 & 0.47 & 2 & 2 & 0 & 0 \\
\hline DISvpCC2pi & 4.2 & 4.2 & 2.4 & 2.4 & 1.5 & 1.5 & 3.6 & 3.6 & 0 & 0 \\
\hline DISvpCC3pi & 3 & 3 & 1.7 & 1.7 & 0.88 & 0.88 & 3 & 3 & 0 & 0 \\
\hline DISvpNCOpi & 0 & 0 & 1.8 & 1.8 & 0 & 0 & 0 & 0 & 4.2 & 4.2 \\
\hline DISvpNC1pi & 0 & 0 & 0.84 & 0.84 & 0 & 0 & 0 & 0 & 2 & 2 \\
\hline DISvpNC2pi & 0 & 0 & 2.7 & 2.7 & 0 & 0 & 0 & 0 & 6.4 & 6.4 \\
\hline DISvpNC3pi & 0 & 0 & 2.2 & 2.2 & 0 & 0 & 0 & 0 & 5.3 & 5.3 \\
\hline DISvnCCOpi & 0.49 & 0.49 & 0.28 & 0.28 & 0.11 & 0.11 & 0.53 & 0.53 & 0 & 0 \\
\hline DISvnCC1pi & 5.6 & 5.6 & 3.2 & 3.2 & 2 & 2 & 4.7 & 4.7 & 0 & 0 \\
\hline DISvnCC2pi & 4.4 & 4.4 & 2.5 & 2.5 & 1.3 & 1.3 & 4.1 & 4.1 & 0 & 0 \\
\hline DISvnCC3pi & 1.7 & 1.7 & 1 & 1 & 0.44 & 0.44 & 6 & 6 & 0 & 0 \\
\hline DISvnNCOpi & 0 & 0 & 0.21 & 0.21 & 0 & 0 & 0 & 0 & 0.5 & 0.5 \\
\hline DISvnNC1pi & 0 & 0 & 2.7 & 2.7 & 0 & 0 & 0 & 0 & 6.4 & 6.4 \\
\hline DISvnNC2pi & 0 & 0 & 2 & 2 & 0 & 0 & 0 & 0 & 4.8 & 4.8 \\
\hline DISvnNC3pi & 0 & 0 & 1.2 & 1.2 & 0 & 0 & 0 & 0 & 2.9 & 2.9 \\
\hline DISvbarpCC0pi & 0.016 & 0.016 & 0.0099 & 0.0099 & 0.026 & 0.026 & 0.099 & 0.099 & 0 & 0 \\
\hline DISvbarpCC1pi & 0.036 & 0.036 & 0.022 & 0.022 & 0.06 & 0.06 & 0.2 & 0.2 & 0 & 0 \\
\hline DISvbarpCC2pi & 0.034 & 0.034 & 0.02 & 0.02 & 0.022 & 0.022 & 0.15 & 0.15 & 0 & 0 \\
\hline DISvbarpCC3pi & 0.01 & 0.01 & 0.006 & 0.006 & 0.011 & 0.011 & 0.041 & 0.041 & 0 & 0 \\
\hline DISvbarpNC0pi & 0 & 0 & 0.086 & 0.086 & 0 & 0 & 0 & 0 & 0.21 & 0.21 \\
\hline DISvbarpNC1pi & 0 & 0 & 0.11 & 0.11 & 0 & 0 & 0 & 0 & 0.26 & 0.26 \\
\hline DISvbarpNC2pi & 0 & 0 & 0.093 & 0.093 & 0 & 0 & 0 & 0 & 0.22 & 0.22 \\
\hline DISvbarpNC3pi & 0 & 0 & 0.067 & 0.067 & 0 & 0 & 0 & 0 & 0.16 & 0.16 \\
\hline DISvbarnCC0pi & 0 & 0 & 0.00012 & 0.00012 & 0.00056 & 0.00056 & 0.015 & 0.015 & 0 & 0 \\
\hline DISvbarnCC1pi & 0.0089 & 0.0089 & 0.0059 & 0.0059 & 0.014 & 0.014 & 0.1 & 0.1 & 0 & 0 \\
\hline DISvbarnCC2pi & 0.03 & 0.03 & 0.018 & 0.018 & 0.03 & 0.03 & 0.032 & 0.032 & 0 & 0 \\
\hline DISvbarnCC3pi & 0.028 & 0.028 & 0.017 & 0.017 & 0.014 & 0.014 & 0.066 & 0.066 & 0 & 0 \\
\hline DISvbarnNC0pi & 0 & 0 & 0.011 & 0.011 & 0 & 0 & 0 & 0 & 0.026 & 0.026 \\
\hline DISvbarnNC1pi & 0 & 0 & 0.077 & 0.077 & 0 & 0 & 0 & 0 & 0.18 & 0.18 \\
\hline DISvbarnNC2pi & 0 & 0 & 0.12 & 0.12 & 0 & 0 & 0 & 0 & 0.27 & 0.27 \\
\hline DISvbarnNC3pi & 0 & 0 & 0.099 & 0.099 & 0 & 0 & 0 & 0 & 0.23 & 0.23 \\
\hline radcorrnue & 2 & 2 & 1.1 & 1.1 & 0 & 0 & 2 & 2 & 0 & 0 \\
\hline radcorrnuebar & 0.017 & 0.017 & 0.0099 & 0.0099 & 0 & 0 & 0.05 & 0.05 & 0 & 0 \\
\hline 2ndclasscurr & 2 & 2 & 1.1 & 1.1 & 0 & 0 & 1.9 & 1.9 & 0 & 0 \\
\hline MaNCEL & 0 & 0 & 0.5 & 0.23 & 0 & 0 & 0 & 0 & 1.2 & 0.56 \\
\hline EtaNCEL & 0 & 0 & 0.004 & 0.0036 & 0 & 0 & 0 & 0 & 0.0095 & 0.0085 \\
\hline VecFFCCQEshape & 0.3 & 0.3 & 0.17 & 0.17 & 0.23 & 0.23 & 0.23 & 0.22 & 0 & 0 \\
\hline MaCCRES & 15 & 12 & 8.3 & 7.1 & 8 & 8.6 & 10 & 9 & 0 & 0 \\
\hline MvCCRES & 8.3 & 7 & 4.8 & 4 & 4.5 & 4.2 & 5.9 & 5.1 & 0 & 0 \\
\hline MaNCRES & 0 & 0 & 5.7 & 4.5 & 0 & 0 & 0 & 0 & 14 & 11 \\
\hline MvNCRES & 0 & 0 & 1.3 & 1.1 & 0 & 0 & 0 & 0 & 3.2 & 2.7 \\
\hline $\mathrm{MaCOHpi}$ & 0.83 & 0.87 & 0.53 & 0.56 & 1.3 & 1.4 & 1 & 1.1 & 0.13 & 0.13 \\
\hline R0COHpi & 0.85 & 0.83 & 0.55 & 0.53 & 1.3 & 1.3 & 1 & 1 & 0.13 & 0.13 \\
\hline
\end{tabular}

TABLE C.3: Shows the $\pm 1 \sigma \%$ shifts for all the GENIE systematic parameters for the $\nu_{\mu}$ selected sample. Continued in the next page. 


\begin{tabular}{|c|c|c|c|c|c|c|c|c|c|c|}
\hline \multirow{2}{*}{ Syst.Parameter } & \multicolumn{2}{|c|}{ App. $\nu_{e}$} & \multicolumn{2}{|c|}{ Total bkg } & \multicolumn{2}{|c|}{$\nu_{\mu}$} & \multicolumn{2}{|c|}{ beam $\nu_{e}$} & \multicolumn{2}{|c|}{$\mathrm{NC}$} \\
\hline & $+1 \sigma(\%)$ & $-1 \sigma(\%)$ & $+1 \sigma(\%)$ & $-1 \sigma(\%)$ & $+1 \sigma(\%)$ & $-1 \sigma(\%)$ & $+1 \sigma(\%)$ & $-1 \sigma(\%)$ & $+1 \sigma(\%)$ & $-1 \sigma(\%)$ \\
\hline AhtBY & 0.43 & 0.44 & 0.52 & 0.53 & 0.068 & 0.07 & 0.64 & 0.65 & 0.64 & 0.65 \\
\hline BhtBY & 0.68 & 0.72 & 0.81 & 0.85 & 0.11 & 0.11 & 0.99 & 1.1 & 1 & 1 \\
\hline CV1uBY & 0.27 & 0.3 & 0.4 & 0.44 & 0.046 & 0.052 & 0.4 & 0.44 & 0.58 & 0.64 \\
\hline CV2uBY & 0.26 & 0.26 & 0.39 & 0.39 & 0.045 & 0.045 & 0.38 & 0.38 & 0.56 & 0.56 \\
\hline NormDISCC & 0 & 0 & 0 & 0 & 0 & 0 & 0 & 0 & 0 & 0 \\
\hline RnubarnuCC & 0 & 0 & 0 & 0 & 0 & 0 & 0 & 0 & 0 & 0 \\
\hline DISNuclMod & 0 & 0 & 0 & 0 & 0 & 0 & 0 & 0 & 0 & 0 \\
\hline- & 0 & 0 & 0 & 0 & 0 & 0 & 0 & 0 & 0 & 0 \\
\hline AGKYxF1pi & 0.00079 & 0.048 & 0.0034 & 0.035 & 0.0015 & 0.0083 & 0.021 & 0.095 & 0.0065 & 0.015 \\
\hline AGKYpT1pi & 0.013 & 0.018 & 0.0085 & 0.015 & 0.0027 & 0.0026 & 0.042 & 0.12 & 0.0021 & 0.0092 \\
\hline FormZone & 4.1 & 6.5 & 4.4 & 7.2 & 0.88 & 1.3 & 3 & 6.2 & 5 & 8.3 \\
\hline MFP_pi & 3.1 & 3.1 & 3.1 & 3.1 & 1.3 & 1.2 & 1.9 & 2 & 3.1 & 3.2 \\
\hline MFP_N & 1.8 & 2 & 1.5 & 1.7 & 2.1 & 2.2 & 1.8 & 2 & 1 & 1.2 \\
\hline FrCEx_pi & 0.12 & 0.11 & 0.078 & 0.072 & 0.12 & 0.12 & 0.17 & 0.15 & 0.025 & 0.021 \\
\hline FrElas_pi & 0.67 & 0.67 & 0.7 & 0.7 & 0.024 & 0.024 & 0.68 & 0.67 & 0.74 & 0.74 \\
\hline FrInel_pi & 1.6 & 1.5 & 1.8 & 1.7 & 0.096 & 0.095 & 1.5 & 1.4 & 1.9 & 1.9 \\
\hline FrAbs_pi & 1.9 & 1.9 & 2.2 & 2.2 & 0.25 & 0.25 & 1.7 & 1.7 & 2.6 & 2.6 \\
\hline FrPiProd_pi & 0.025 & 0.025 & 0.038 & 0.038 & 0.005 & 0.005 & 0.052 & 0.053 & 0.12 & 0.12 \\
\hline FrCEx_N & 0.03 & 0.031 & 0.0095 & 0.0088 & 0.12 & 0.12 & 0.32 & 0.32 & 0.057 & 0.056 \\
\hline FrElas_N & 0.67 & 0.67 & 0.44 & 0.44 & 0.25 & 0.25 & 1 & 1 & 0.11 & 0.11 \\
\hline FrInel_N & 0.16 & 0.16 & 0.13 & 0.14 & 0.031 & 0.03 & 0.27 & 0.27 & 0.097 & 0.098 \\
\hline FrAbs_N & 0.42 & 0.42 & 0.29 & 0.29 & 0.047 & 0.047 & 0.25 & 0.25 & 0.11 & 0.12 \\
\hline FrPiProd_N & 0.079 & 0.079 & 0.025 & 0.025 & 0.0033 & 0.0033 & 0.042 & 0.042 & 0.17 & 0.17 \\
\hline CCQEPauliSupViaKF & 0.054 & 0.053 & 0.031 & 0.031 & 1.4 & 1.3 & 0.078 & 0.08 & 0 & 0 \\
\hline CCQEMomDistroFGtoSF & 0 & 0 & 0 & 0 & 0 & 0 & 0 & 0 & 0 & 0 \\
\hline RDecBR1gamma & 0.01 & 0.01 & 0.013 & 0.013 & 0.0018 & 0.0018 & 0.012 & 0.012 & 0.016 & 0.016 \\
\hline RDecBR1eta & 0.57 & 0.57 & 0.7 & 0.7 & 0.44 & 0.44 & 0.79 & 0.79 & 0.88 & 0.88 \\
\hline Theta_Delta2Npi & 1.6 & 1.1 & 1.2 & 0.79 & 1.3 & 0.88 & 1.2 & 0.81 & 0.61 & 0.41 \\
\hline
\end{tabular}

TABlE C.4: Shows the $\pm 1 \sigma \%$ shifts for the remaining GENIE systematic parameters for the $\nu_{\mu}$ selected sample. 


\section{C.3 Effect of GENIE Parameters on Energy Distribution}
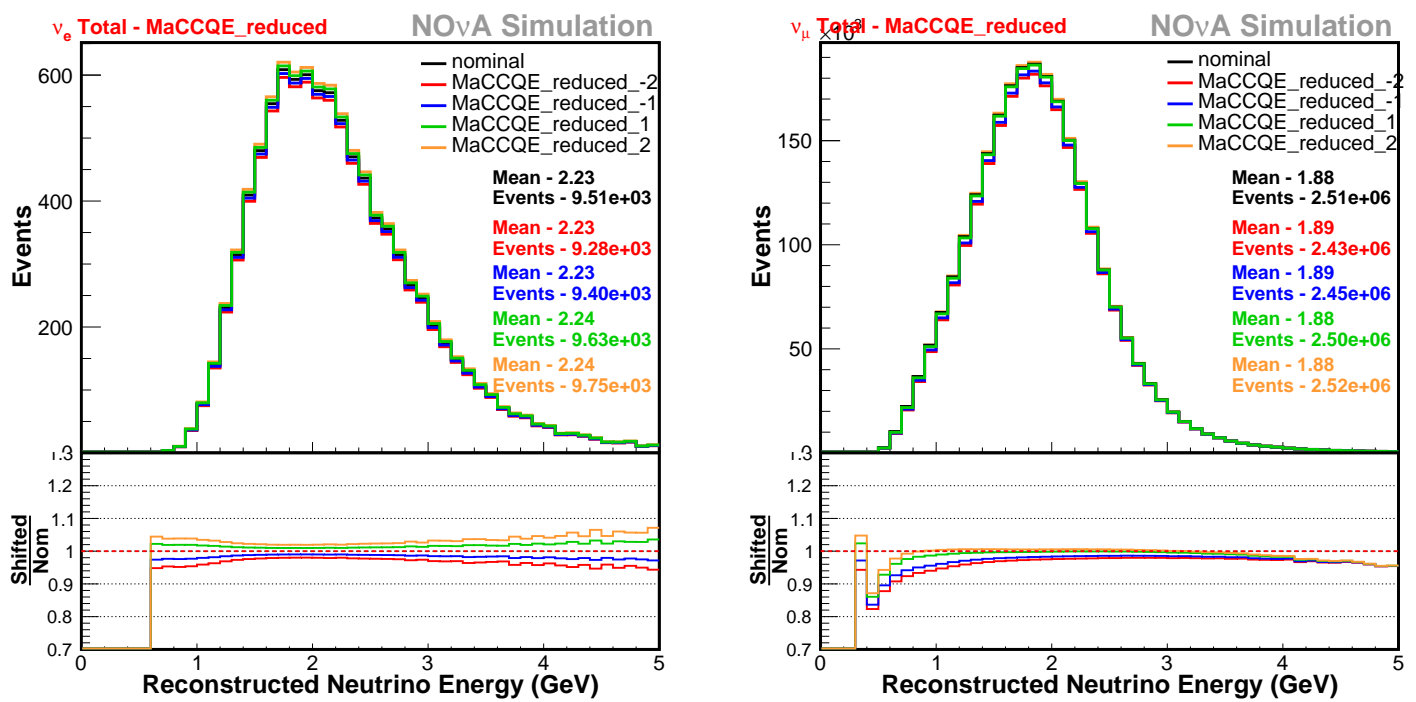

FiguRE C.1: Altra figura experimental
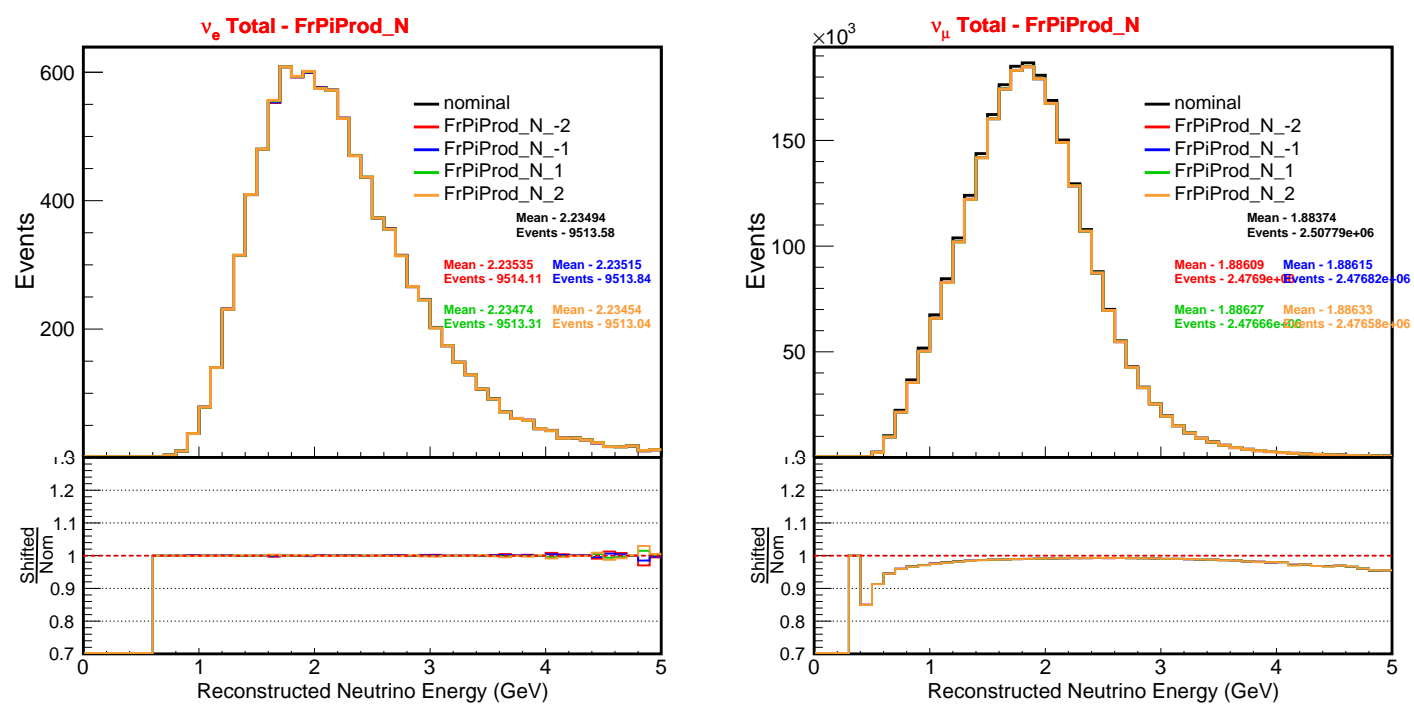

FiguRE C.2: Shows the effect of GENIE parameter FrPiProd_N on both $\nu_{e}$ (left) and $\nu_{\mu}$ selected sample which doesn't include appeared $\nu_{e}$ 's. 

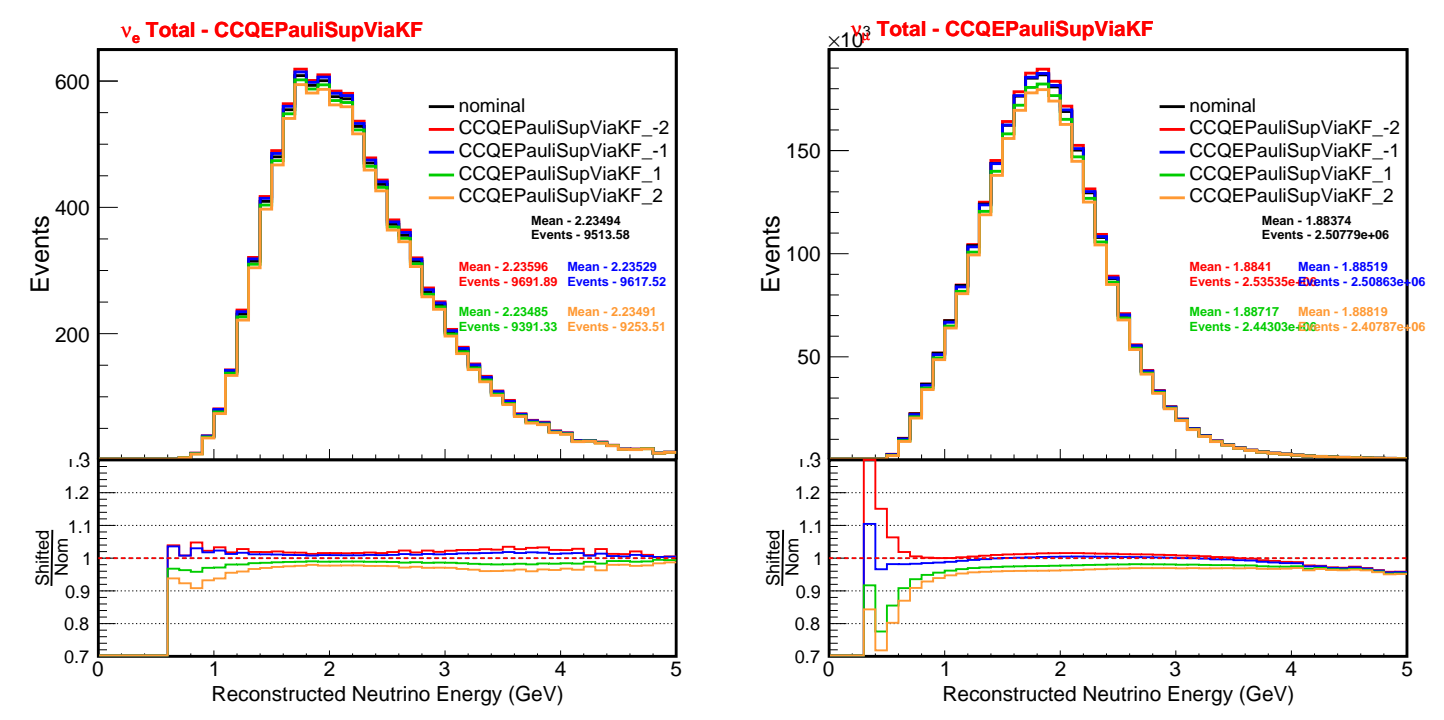

Figure C.3: Shows the effect of GENIE parameter CCQEPauliSupViaKF on both $\nu_{e}$ (left) and $\nu_{\mu}$ selected sample which doesn't include appeared $\nu_{e}$ 's. 

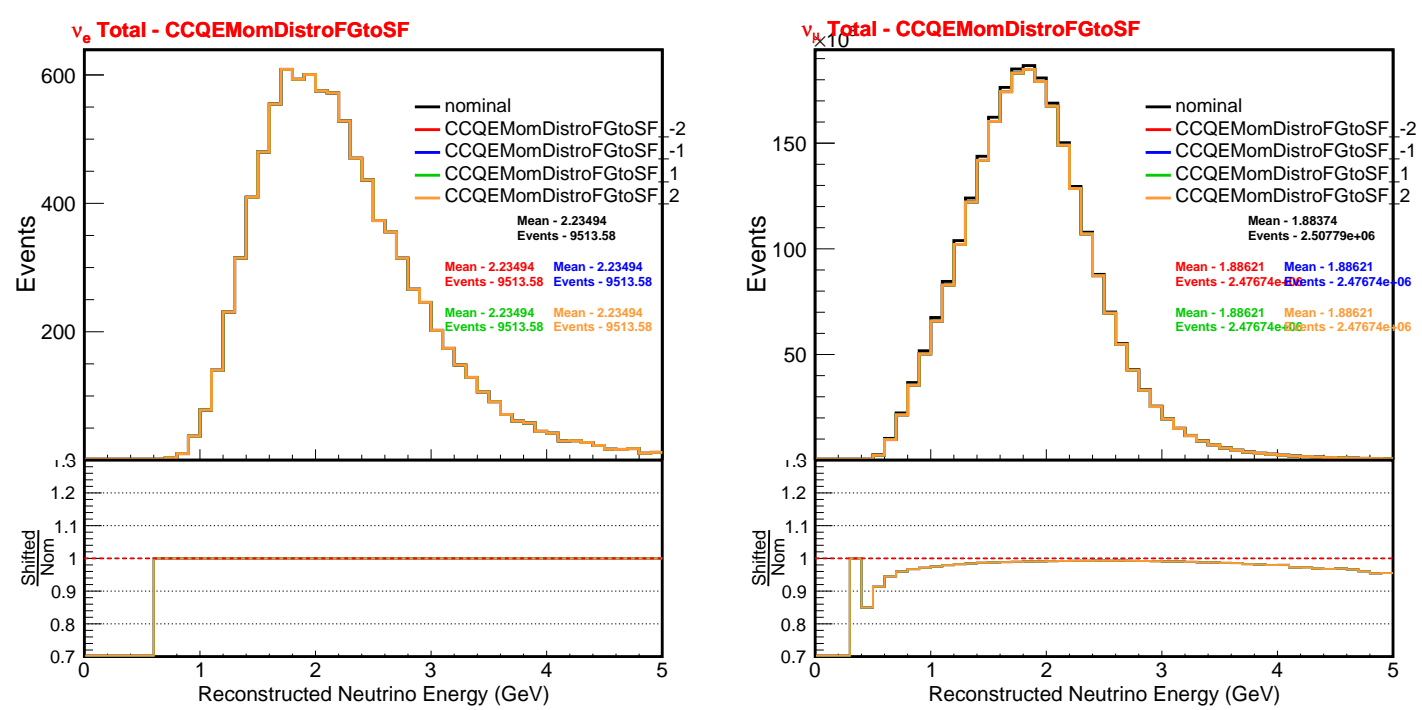

Figure C.4: Shows the effect of GENIE parameter CCQEMomDistroFGtoSF on both $\nu_{e}$ (left) and $\nu_{\mu}$ selected sample which doesn't include appeared $\nu_{e}$ 's.
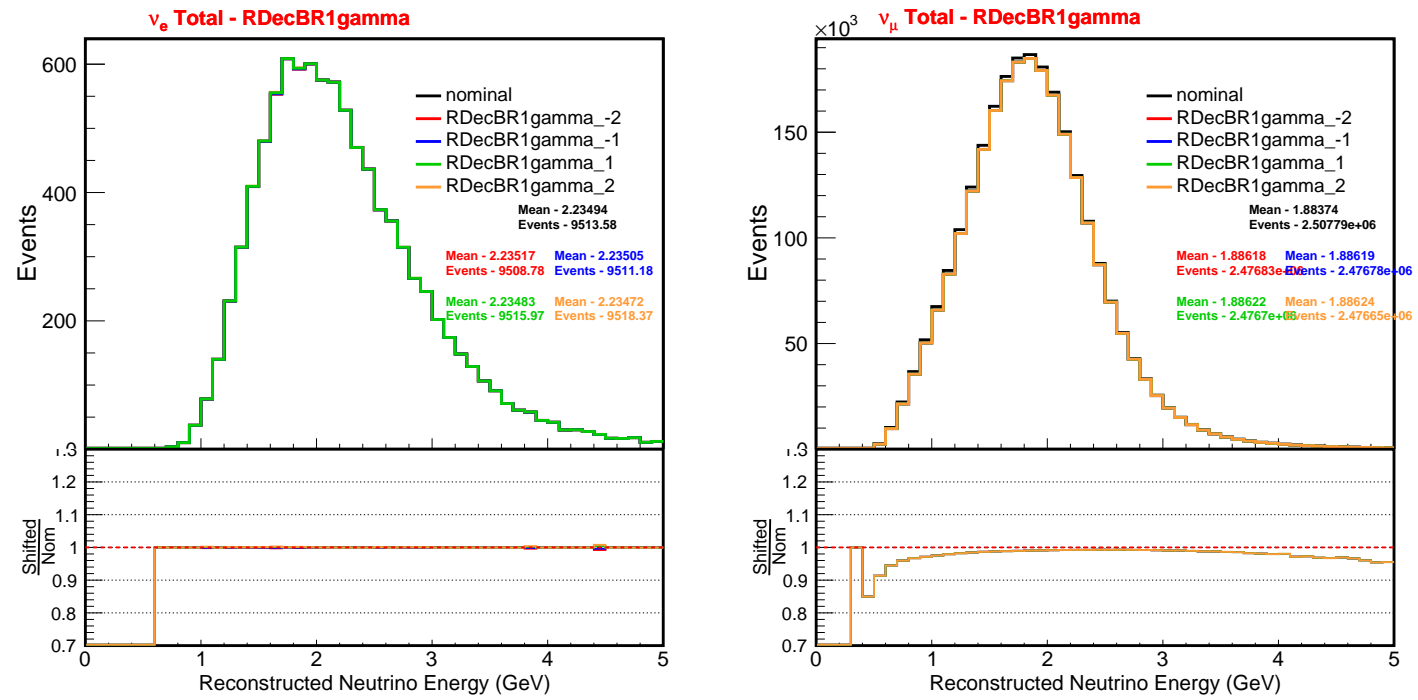

FIGURE C.5: Shows the effect of GENIE parameter RDecBR1gamma on both $\nu_{e}$ (left) and $\nu_{\mu}$ selected sample which doesn't include appeared $\nu_{e}$ 's. 

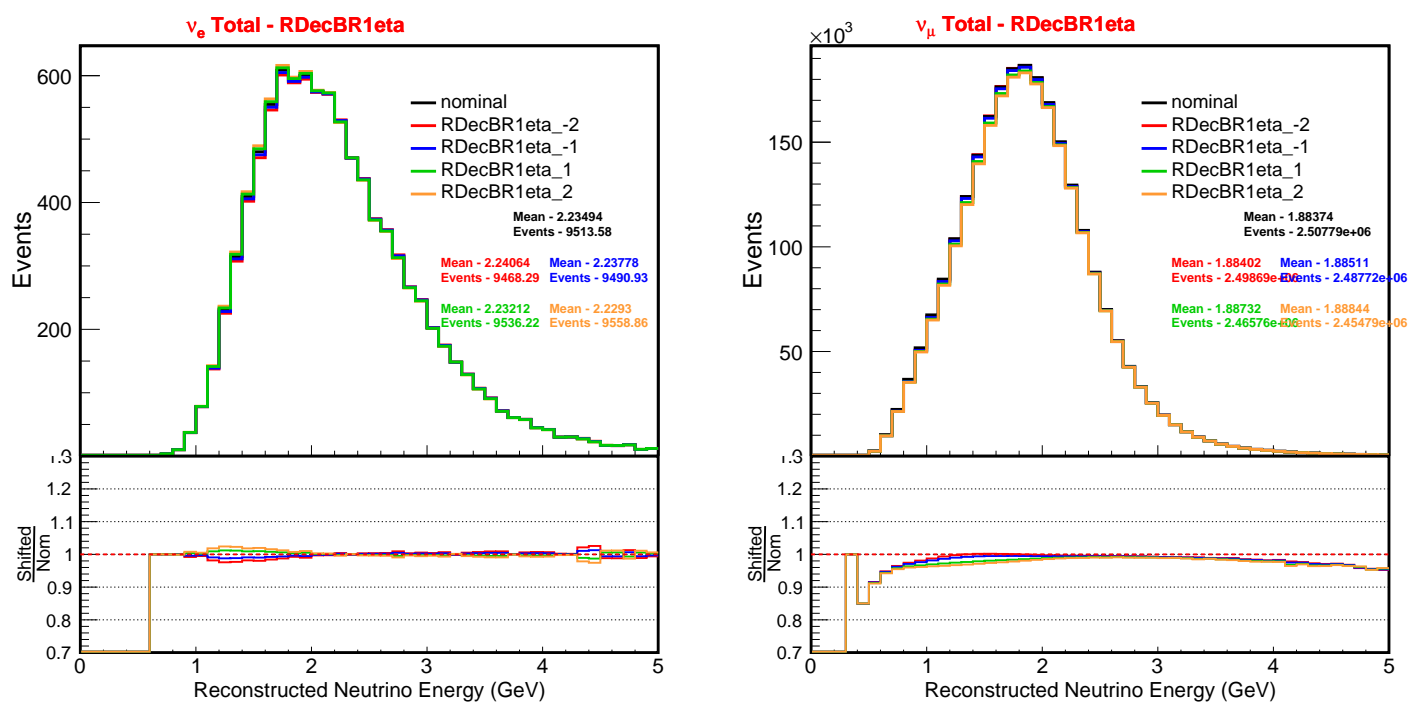

Figure C.6: Shows the effect of GENIE parameter RDecBR1eta on both $\nu_{e}$ (left) and $\nu_{\mu}$ selected sample which doesn't include appeared $\nu_{e}$ 's.
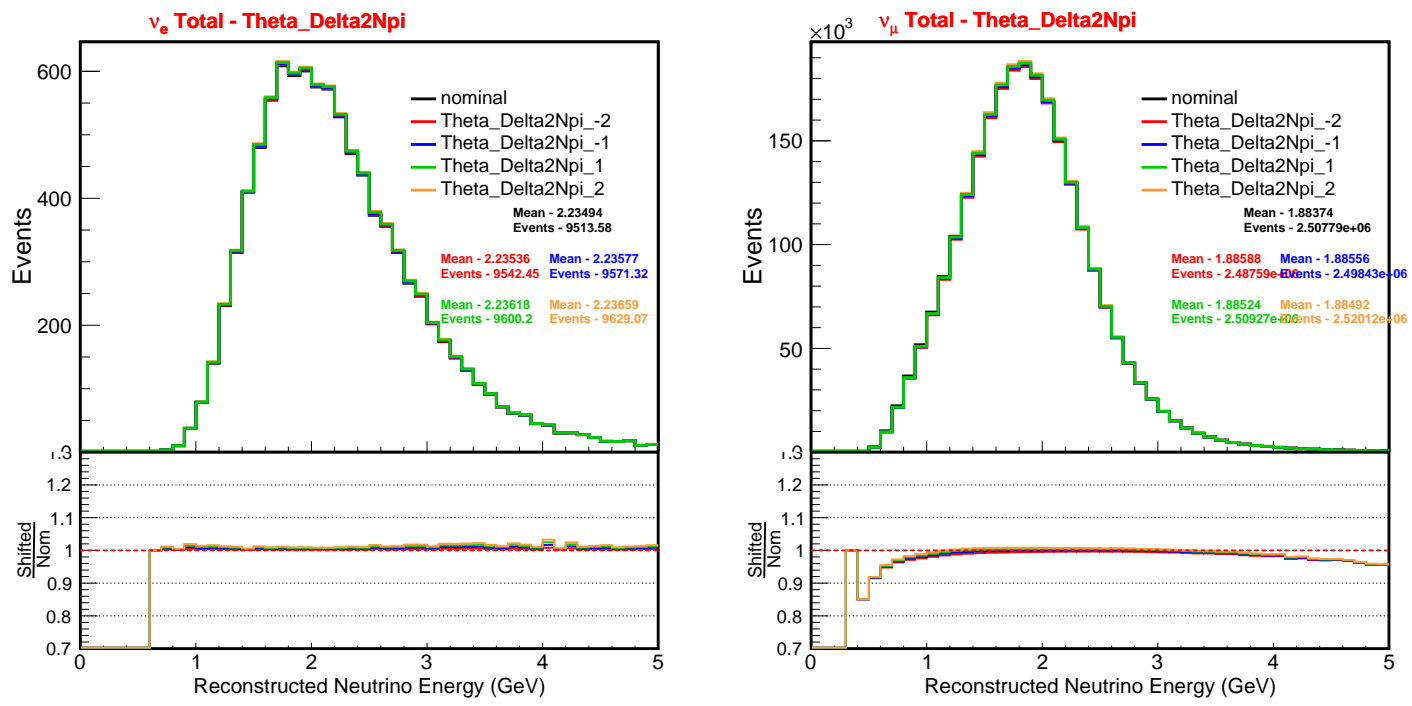

FiguRE C.7: Shows the effect of GENIE parameter Theta_Delta2Npi on both $\nu_{e}$ (left) and $\nu_{\mu}$ selected sample which doesn't include appeared $\nu_{e}$ 's. 


\section{Bibliography}

[1] Becquerel, H, "Sur les radiations invisibles mises par les corps phosphorescents," Comptes rendus de l Acadmie des Sciences Paris 122 (1896) 501.

[2] Becquerel, H, "Sur quelques proprits nouvelles des radiations invisibles mises par divers corps phosphorescents," Comptes rendus de l Acadmie des Sciences Paris 122 (1896) 559.

[3] Becquerel, H, "Sur les radiations invisibles mises par les sels duranium," Comptes rendus de l Acadmie des Sciences Paris 122 (1896) 689.

[4] Becquerel, H, "Sur les proprits diffrentes des radiations invisibles mises par les sels duranium, et du rayonnement de la paroi anticathodique dun tube de Crookes," Comptes rendus de l Acadmie des Sciences Paris 122 (1896) 762.

[5] Curie, M, "Rayons mis par les composes de luranium et du thorium," Comptes rendus de 1 Acadmie des Sciences Paris 126 (1898) 1101-1103.

[6] J. Chadwick, "Possible Existence of a Neutron," Nature 129 (1932) 312.

[7] C. D. Ellis, W. A. Wooster, "The average energy of disintegration of radium E," Proceedings of the Royal Society of London A: Mathematical, Physical and Engineering Sciences 117 (1927) no. 776, 109-123, http://rspa.royalsocietypublishing.org/content/117/776/109.full.pdf.

[8] W. Pauli, "On the Earlier and more recent history of the neutrino," Cambridge Monogr. Part. Phys. Nucl. Phys. Cosmol. 14 (2000) 1-22.

[9] E. Fermi, "An attempt of a theory of beta radiation," Z. Phys. 88 (1934) 161-177.

[10] G. Gamow and E. Teller, "Selection Rules for the $\beta$-Disintegration," Phys. Rev. 49 (1936) 895-899.

[11] J. C. Street and E. C. Stevenson, "New Evidence for the Existence of a Particle of Mass Intermediate Between the Proton and Electron," Phys. Rev. 52 (1937) 1003-1004. 
[12] Lee, T.D. and Yang, C.N., "Question of parity conservation in weak interactions," Phys. Rev. 104 (1956) 254-258.

[13] Wu, C.S., E. Ambler, R. W. Hayward, D. D. Hoppes, and R. P. Hudson, "Experimental test of parity conservation in beta decay," Phys. Rev. 105 (1957) 1413-1415.

[14] E. C. G. Sudarshan and R. E. Marshak, "Chirality Invariance and the Universal Fermi Interaction," Phys. Rev. 109 (1958) 1860.

[15] R. P. Feynman and M. Gell-Mann, "Chirality Invariance and the Universal Fermi Interaction," Phys. Rev. 109 (1958) 193-198.

[16] F. Reines, C. L. Cowan, F. B. Harrison, A. D. McGuire, and H. W. Kruse, "Detection of the Free Antineutrino," Phys. Rev. 117 (1960) 159-173.

[17] G. Danby et al., "Observation of High-Energy Neutrino Reactions and the Existence of Two Kinds of Neutrinos," Phys. Rev. Lett. 9 (1962) 36-44.

[18] C. Giunti and Chung W. Kim, Fundamental of neutrino Physics and Astrophysics. Oxford University Press, 1997.

[19] Cooper, N.G., "Celebrating the neutrino.," Tech. Rep. 25, 1997.

[20] http://prospect.yale.edu/science\#Neutrinos.

[21] B. Pontecorvo, "Neutrino Experiments and the Problem of Conservation of Leptonic Charge," JETP 26 (1968) no. 5, 984-988.

[22] B. T. Cleveland et al., "Measurement of the Solar Electron Neutrino Flux with the Homestake Chlorine Detector," Astrophys.J 496 (1998) 505-526.

[23] Kamiokande, K.S. Hirata et al., "Observation of ${ }^{8} B$ solar neutrinos in the Kamiokande-II detector," Phys. Rev. Lett. 63 (1989) 16.

[24] Kamiokande, Y. Fukuda et al., "Solar Neutrino Data Covering Solar Cycle 22," Phys. Rev. Lett. 77 (1996) 1683.

[25] KamLAND, K. Eguchi et al., "Determination of solar neutrino oscillation parameters using 1496 days of Super-Kamiokande I data," Phys. Rev. Lett. 90 (2003) 021802.

[26] KamLAND, T. Araki et al., "Measurement of Neutrino Oscillation with KamLAND: Evidence of Spectral Distortion," Phys. Rev. Lett. 94 (2005) 081801.

[27] S. Eliezer and A. R. Swift, "Experimental consequences of $\nu_{e}-\nu_{\mu}$ mixing for neutrino beams," Nucl. Phys. B105 (1976) 45. 
[28] H. Fritzsch and P. Minkowski, "Vector-Like Weak Currents, Massive Neutrinos, and Neutrino Beam Oscillations," Phys. Lett. B62 (1976) 72-76.

[29] S. M. Bilenky and B. Pontecorvo, "Lepton mixing and neutrino oscillations," Phys. Rep. 41 (1978) 225-261.

[30] Z. Maki, M. Nakagawa, and S. Sakata, "Remarks on the Unified Model of Elementary Particles," Prog. Theor. Phys. 28 (1962) 870.

[31] Super-Kamiokande, S. Fukuda et al., "Determination of solar neutrino oscillation parameters using 1496 days of Super-Kamiokande I data," Phys. Lett. B539 (2002) 179-187.

[32] SNO, Q.R. Ahmad et al., "Measurement of the Rate of $\nu_{e}+d \rightarrow p+p+e^{-}$ Interactions Produced by ${ }^{8} B$ Solar Neutrinos at the Sudbury Neutrino ," Phys. Rev. Lett. 87 (2001) 071301.

[33] SNO, Q.R. Ahmad et al., "Direct Evidence for Neutrino Flavor Transformation from Neutral-Current Interactions in the Sudbury Neutrino Observatory," Phys. Rev. Lett. 89 (2002) 011301.

[34] SAGE, J. N. Abdurashitov et al., "The Russian-American Gallium Experiment (SAGE) Cr Neutrino Source Measurement," Phys. Rev. Lett. 77 (1996) 4708.

[35] SAGE, J. N. Abdurashitov et al., "Measurement of the response of a gallium metal solar neutrino experiment to neutrinos from a ${ }^{51} \mathrm{Cr}$ source," Phys. Rev. C59 (1999) 2246.

[36] SAGE, J. N. Abdurashitov et al., "Measurement of the response of a Ga solar neutrino experiment to neutrinos from a ${ }^{37}$ Ar source," Phys. Rev. C73 (2006) 045805 .

[37] SAGE, J. N. Abdurashitov et al., "Measurement of the solar neutrino capture rate with gallium metal. III: Results for the 2002-2007 data-taking period," Phys. Rev. C80 (2009) 015807.

[38] SAGE, J.N. Abdurashitov et al., " Measurement of the solar neutrino capture rate with gallium metal. III: Results for the 2002-2007 data-taking period," Phys. Rev. C80 (2009) 015807.

[39] GALLEX, P. Anselmann et al., "Solar neutrinos observed by GALLEX at Gran Sasso," Phys. Lett. B285 (1992) 376-389.

[40] GALLEX, W. Hampel et al., "GALLEX solar neutrino observations: results for GALLEX IV," Phys. Lett. B447 (1999) 127-133. 
[41] GALLEX, P. Anselmann et al., "First results from the ${ }^{51} \mathrm{Cr}$ neutrino source experiment with the GALLEX detector," Phys. Lett. B342 (1995) 440-450.

[42] GALLEX, W. Hampel et al., "Final results of the ${ }^{51} \mathrm{Cr}$ neutrino source experiments in GALLEX," Phys. Lett. B420 (1998) 114-126.

[43] GALLEX, P. Anselmann et al., "Solar Neutrino Data Covering Solar Cycle 22," Phys. Lett. B285 (1992) 376.

[44] GNO, M. Altmann et al., "Complete results for five years of GNO solar neutrino observations," Phys. Lett. B616 (2005) 174.

[45] Borexino, G. Bellini et al., "Precision Measurement of the ${ }^{7}$ Be Solar Neutrino Interaction Rate in Borexino," Phys. Rev. Lett. 107 (2011) 141302.

[46] Borexino, G. Bellini et al., "First Evidence of pep Solar Neutrinos by Direct Detection in Borexino," Phys. Rev. Lett. 108 (2012) 051302.

[47] Borexino, G. Bellini et al., "Neutrinos from the primary protonproton fusion process in the Sun," Nature $\mathbf{5 1 2}$ (2014) 383.

[48] J.N. Bahcall, A.M. Serenelli, and S. Basu, "New solar opacities, abundances, helioseismology and neutrino fluxes," Astrophys. J. 621 (2005) L85.

[49] Davide D’Angelo, “Low Energy Neutrino Measurements," arXiv:1211.5359.

[50] IMB, D. Casper et al., "Measurement of atmospheric neutrino composition with the IMB-3 detector," Phys. Rev. Lett. 66 (1991) 2561.

[51] MINOS, D.G. Michael et al., "Observation of Muon Neutrino Disappearance with the MINOS Detectors in the NuMI Neutrino Beam," Phys. Rev. Lett. 97 (2006) 191801.

[52] MINOS, P. Adamson et al., "Measurement of Neutrino Oscillations with the MINOS Detectors in the NuMI Beam," Phys. Rev. Lett. 101 (2008) 131802.

[53] MINOS, P. Adamson et al., "Measurement of the Neutrino Mass Splitting and Flavor Mixing by MINOS," Phys. Rev. Lett. 106 (2011) 181801.

[54] MACRO, M. Ambrosio et al., "Measurement of the atmospheric neutrino-induced upgoing muon flux using MACRO," Phys. Lett. B434 (1998) 451.

[55] ANTARES, M. Ageron et al., "ANTARES: the first undersea neutrino telescope," Nucl. Instrum. Methods A656 (2011) 11. 
[56] K2K, M.H. Ahn et al., "Measurement of neutrino oscillation by the K2K experiment," Phys. Rev. D74 (2006) 072003.

[57] M. Apollonio et al., "Search for neutrino oscillations on a long base-line at the CHOOZ nuclear power station," Eur. Phys. J. C27 (2003) 331, arXiv:0301017.

[58] F. Ardellier et al., "Letter of Intent for Double-CHOOZ: a Search for the Mixing Angle Theta13," arXiv:0405032.

[59] Daya Bay, F. P. An et al., "Observation of Electron-Antineutrino Disappearance at Daya Bay," Phys. Rev. Lett. 108 (2012) 171803.

[60] Daya Bay, F. P. An et al., "Spectral Measurement of Electron Antineutrino Oscillation Amplitude and Frequency at Daya Bay," Phys. Rev. Lett. 112 (2014) 061801.

[61] RENO, J. H. Choi et al., "Observation of Energy and Baseline Dependent Reactor Antineutrino Disappearance in the RENO Experiment," Phys. Rev. Lett. 116 (2016) 211801, arXiv:1511.05849.

[62] F. Boehm et al., "Search for neutrino oscillations on a long base-line at the CHOOZ nuclear power station," Phys. Rev. D64 (2001) 112001, arXiv:0107009.

[63] MINOS+, G. Tzanakos et al., "MINOS+: a Proposal to FNAL to run MINOS with the medium energy NuMI beam," FERMILAB-PROPOSAL-1016 (2011) .

[64] MINOS+, P. Adamson et al., "Neutrinos from the primary protonproton fusion process in the Sun," arXiv:1710.06488.

[65] T2K, K. Abe et al., "First muon-neutrino disappearance study with an off-axis beam," Phys. Rev. D85 (2012) 031103.

[66] T2K, K. Abe et al., "Measurement of Neutrino Oscillation Parameters from Muon Neutrino Disappearance with an Off-Axis Beam," Phys. Rev. Lett. 111 (2013) 211803.

[67] NOvA, P. Adamson et al., "First measurement of muon-neutrino disappearance in NOvA," Phys. Rev. D93 (2016) 051104.

[68] NOvA, P. Adamson et al., "First Measurement of Electron Neutrino Appearance in NOvA," Phys. Rev. Lett. 116 (2016) 151806.

[69] OPERA, N. Agafonova et al., "Observation of a first $\nu_{\tau}$ candidate event in the OPERA experiment in the CNGS beam," Phys. Lett. B691 (2010) 138. 
[70] OPERA, N. Agafonova et al., "New results on $\nu_{\mu} \rightarrow \nu_{\tau}$ appearance with the OPERA experiment in the CNGS beam," JHEP 1311 (2013) 036.

[71] M.C. Gonzalez-Garcia et al., "Global Analyses of Neutrino Oscillation Experiments," Nuclear Physics B B00 (2015) 1-16.

[72] C. Patrignani et al., "Review of Particle Physics," Chin. Phys. C40 (2016) 100001.

[73] M. Archidiacono, N. Fornengo, C. Giunti, S. Hannestad, and A. Melchiorri, "Sterile Neutrinos: Cosmology vs Short-BaseLine Experiments," Phys. Rev. D87 (2013) 125034, arXiv:1302.6720.

[74] J. Hamann et al., "Cosmology seeking friendship with sterile neutrinos," Phys. Rev. Lett. 105 (2010) 181301, arXiv:1006.5276.

[75] PLANCK, P. Ade et al., "Planck 2013 results. XVI. Cosmological parameters," Astronomy \& Astrophysics 571 (2014) no. A16, , arXiv:1303.5076.

[76] LSND, A. Aguilar et al., "Evidence for neutrino oscillations from the observation of $\bar{\nu}_{e}$ appearance in a $\bar{\nu}_{\mu}$ beam," Phys. Rev. D64 (2001) 112007.

[77] MiniBooNE, A. Aguilar-Arevalo et al., "Improved Search for $\bar{\nu}_{\mu} \rightarrow \bar{\nu}_{e}$ Oscillations in the MiniBooNE Experiment," Phys. Rev. Lett. 110 (2013) 161801.

[78] Y. Declais, et al., "Study of reactor anti-neutrino interaction with proton at Bugey nuclear power plant," Phys. Lett. B338 (1994) 383-389.

[79] B. Achkar, et al., "Search for neutrino oscillations at 15-meters, 40-meters, and 95-meters from a nuclear power reactor at Bugey," Nucl. Phys B434 (1995) 503-534.

[80] A.A. Kuvshinnikov, L.A. Mikaelyan, S.V. Nikolaev, M.D. Skorokhvatov, A.V. Etenko, "Measuring the anti-electron-neutrino $+p \rightarrow n+e^{+}$cross-section and beta decay axial constant in a new experiment at Rovno NPP reactor," JETP Lett. 54 (1991) 253-257.

[81] P. Vogel, "Analysis of the antineutrino capture on protons," Phys. Rev. D29 (1984) 1918.

[82] A. Hoummada, S.L. Mikou, M. Avenier, G. Bagieu, J. Cavaignac, D.H. Koang, "Neutrino oscillations I.L.L. experiment reanalysis," Appl. Radiat. Isot 46 (1995) no. 6-7, 449-450. 
[83] G. Zacek, et al., "Neutrino oscillation experiments at the Gosgen nuclear power reactor," Phys. Rev. D34 (1986) 2621-2636.

[84] G.S. Vidyakin, et al., "Bounds on the neutrino oscillation parameters for reactor anti-neutrinos," Sov. Phys. JETP71 (1990) 424-426.

[85] Z.D. Greenwood, et al., "Results of a two position reactor neutrino oscillation experiment," Phys. Rev. D53 (1996) 6054-6064.

[86] G. Mention et al., "Reactor antineutrino anomaly," Phys. Rev. D83 (2011) 073006 .

[87] J. Kopp, P. A. N. Machado, M. Maltoni, and T. Schwetz, "Sterile neutrino oscillations: the global picture," J. High Energy Phys. 50 (2013) 2013.

[88] S. Gariazzo, C. Giunti, M. Laveder, and Y. Li, "Updated global 3+1 analysis of short-baseline neutrino oscillations," J. High Energy Phys. 06 (2017) 135.

[89] NOvA, "NOvA Technical Design Report." https://www-nova.fnal.gov/nova_cd2_review/tdr_oct_23/tdr.htm.

[90] "MONTE CARLO TECHNIQUES,".

[91] Andreopoulos C et al., "The GENIE Neutrino Monte Carlo Generator," Nucl. Instrum. Meth. A614 (2010) 87-104.

[92] "A very fast random number generator." http://www .math.sci.hiroshima-u.ac.jp/ m-mat/MT/emt.html.

[93] Daniel Kodroff, Michael Kordosky, "An Investigation on Improving Neutrino Flux Simulations from the NuMI Beamline at Fermilab," tech. rep.

[94] "Model: FTFP." http:

//geant4.cern.ch/support/proc_mod_catalog/models/hadronic/FTFP.html.

[95] "Model: Bertini cascade." http://geant4.cern.ch/support/proc_mod_ catalog/models/hadronic/BertiniCascade.html.

[96] Hagmann C, Lange D and Wright D, "Determination of pion intranuclear rescattering rates in $\nu_{\mu}$ Ne versus $\nu_{\mu} D$ interactions for the atmospheric neutrino flux," IEEE Nuclear Science Symp. Conf. Rec. (Honolulu, HI) 2 (2007) $1143-1146$.

[97] Kanika Sachdev, Muon Neutrino To Electron Neutrino Oscillation in NOvA. $\mathrm{PhD}$ thesis, 2015. 
[98] J.B. Birks, "Scintillations from organic crystals: specific fluorescence and relative response to different radiations," Proc. Phys. Soc. A64 (1951) 874-877.

[99] C.N. Chou, "The nature of the saturation effect of fluorescent scintillators," Phys. Rev. D87 (1952) 904.

[100] , ",".

[101] Adam Aurisano, "NOvA internal document, Technote: 2017 Light Model." https :

//nova-docdb.fnal.gov/cgi-bin/private/ShowDocument?docid=23228.

[102] Michael Baird, "NOvA Internal Document: A Side By Side Comparison of Slicer, Cosmic Slicer, and Slicer $4 D . "$

https://nova-docdb.fnal.gov/cgi-bin/private/ShowDocument?docid=9195.

[103] Jrg SanderMartin EsterHans-Peter KriegelXiaowei Xu, "Density-Based Clustering in Spatial Databases: The Algorithm GDBSCAN and Its Applications," Data Mining and Knowledge Discovery 2 (1998) 169.

[104] L. Fernandes and M. Oliveira, "Real-time line detection through an improved Hough transform voting scheme," Pattern Recognition 41 (2008) 299-314.

[105] Mark Messier, "NOvA Internal Document: Vertex Reconstruction Based on Elastic Arms."

https://nova-docdb.fnal.gov/cgi-bin/private/ShowDocument?docid=7530.

[106] Evan Niner, "NOvA Internal Document: Vertex Clustering with Possibilistic Fuzzy-K Means Algorithm." https://nova-docdb.fnal.gov/cgi-bin/private/ShowDocument?docid=7648.

[107] J. H. Friedman, "Stochastic gradient boosting," Computational Statistics \& Data Analysis 38 (2002) 367-378.

[108] N. S. Altman, "An Introduction to Kernel and Nearest-Neighbor Nonparametric Regression," American Statistician 46 (1992) 175-185.

[109] F. Rosenblatt, Principles of Neurodynamics: Perceptrons and the Theory of Brain Mechanisms. Spartan Books, 1961.

[110] R. Reed and R. Marks, Neural Smithing: Supervised Learning in Feedforward Artificial Neural Networks. A Bradford book. MIT Press, 1999.

[111] Y. LeCun, Y. Bengio and G. Hinton, "Deep learning," Nature 521 (2015) $436-444$. 
[112] C. Szegedy, W. Liu, Y. Jia, P. Sermanet, S. Reed, D. Anguelov et al., "Going deeper with convolutions," arXiv:1409.4842.

[113] Y. Jia, E. Shelhamer, J. Donahue, S. Karayev, J. Long, R. Girshick et al., "Caffe: Convolutional architecture for fast feature embedding," arXiv:1408.5093.

[114] Y. LeCun, L. Bottou, G. B. Orr and K. R. Mller, Neural Networks: Tricks of the Trade. Springer-Verlag Berlin Heidelberg, 1998.

[115] A. Aurisano, A. Radovic, D. Rocco, A. Himmel, M. Messier, E. Niner, G. Pawloski, F. Psihas, A. Sousa, and P. Vahle, "A convolutional neural network neutrino event classifier," Journal of Instrumentation 11 (2016) no. 09, P09001. http://stacks . iop.org/1748-0221/11/i=09/a=P09001.

[116] Shiqi Yu, Fernanda Psihas, Zelimir Djurcic, "NOvA internal document, Technote: Energy Reconstruction for $2017 \nu_{e}$ Appearance Analysis." https: //nova-docdb.fnal.gov/cgi-bin/private/ShowDocument?docid=22502.

[117] Dmitrii Torbunov, "NOvA internal document, Technote: NuMu Energy Estimator Technote for Prod3 MC." https:

//nova-docdb.fnal.gov/cgi-bin/private/ShowDocument?docid=23342.

[118] Linda Cremonesi, "NOvA internal document: Beam systematic uncertainties for third analyses." http://nova-docdb.fnal.gov:8080/cgi-bin/ShowDocument?docid=17608.

[119] Bannanje Nitish Nayak, "NOvA internal document, A Technote on Hadron Production Systematics for the NOvA Oscillation Analysis." http://nova-docdb.fnal.gov:8080/cgi-bin/ShowDocument?docid=22532.

[120] Denver W. Whittington, "NOvA internal document: Systematic for Short-Track High-y DIS." http://nova-docdb.fnal.gov:8080/cgi-bin/ShowDocument?docid=21725.

[121] Mathew Strait, "NOvA internal document: Muon Energy Scale Systematic." http://nova-docdb.fnal.gov:8080/cgi-bin/ShowDocument?docid=20816.

[122] H. Duyang et al., "NOvA internal document: A Technote Describing the Derivation and Size of NuMI Flux Uncertainties Used in the First NOvA Analyses." http://nova-docdb.fnal.gov:8080/cgi-bin/ShowDocument?docid=13584.

[123] Siva Prasad K, "NOvA internal document: Update on Short-Baseline Analysis." http://nova-docdb.fnal.gov:8080/cgi-bin/ShowDocument?docid=24526. 
[124] Bruno Zamorano, "NOvA internal document: Intensity-related systematic uncertainty for the second analysis."

http://nova-docdb.fnal.gov:8080/cgi-bin/ShowDocument?docid=15217.

[125] Daniel Pershey, "NOvA internal document: Nue Data Stability." http: //nova-docdb.fnal .gov:8080/cgi-bin/ShowDocument?docid=18276.

[126] Christopher Backhouse, "NOvA internal document: The CAFAna Framework." http://nova-docdb.fnal gov: 8080/cgi-bin/ShowDocument?docid=9222.

[127] K.E.Atkinson, SIAM J., “,” J. Numerical Analysis 5 (1968) 89-101.

[128] Gavin S. Davies, "NOvA internal document, A Technote on Single Neutrino Overlays for NOvA Analyses." http://nova-docdb.fnal.gov:8080/cgi-bin/ShowDocument?docid=26334.

[129] Gavin S. Davies, Justin Vasel, "NOvA internal document, A Technote on Cosmic Overlays for NOvA Analyses." http://nova-docdb.fnal .gov:8080/cgi-bin/ShowDocument?docid=13162.

[130] Siva Prasad Kasetti, "NOvA internal document, Technote: Systematic Uncertainties for the 2017 Joint $\nu_{e}$ Appearance and $\nu_{\mu}$ Disappearance Short-Baseline Analysis." https: //nova-docdb.fnal.gov/cgi-bin/private/ShowDocument?docid=26211.

[131] Jeremy Wolcott, Kirk Bays, "NOvA internal document: Cross section tuning $\&$ uncertainties for 2017 analyses." http://nova-docdb.fnal .gov:8080/cgi-bin/ShowDocument?docid=23264.

[132] NOvA, P. Adamson et al., "Constraints on Oscillation Parameters from $\nu_{e}$ Appearance and $\nu_{\mu}$ Disappearance in NOvA," Phys. Rev. Lett. 118 (2017) 231801, arXiv:1703.03328.

[133] Christopher J. Backhouse, Patricia Vahle, "NOvA internal document: Nue Second Analysis Executive Summary." http: //nova-docdb.fnal .gov:8080/cgi-bin/ShowDocument?docid=15397.

[134] NOvA, P. Adamson et al., "Measurement of the neutrino mixing angle $\theta_{23}$ in NOvA," Phys. Rev. Lett. 118 (2017) 151802, arXiv:1701.05891.

[135] Jeff Hartnell, Gregory Pawloski, "NOvA internal document: NuMu Analysis Package Summary for Box-Opening Review May 2016." http: //nova-docdb. fnal gov: 8080/cgi-bin/ShowDocument?docid=15232. 
[136] Lisa Goodenough and Sarah Phan-Budd, "NOvA internal document: Technical Note on the NOvA Beam Monitoring for the 2015 Summer Analysis." http://nova-docdb.fnal.gov:8080/cgi-bin/ShowDocument?docid=13572.

[137] Xuebing Bu and Kanika Sachdev, "NOvA internal document: Spill Level Data Quality." http://nova-docdb.fnal.gov:8080/cgi-bin/ShowDocument?docid=12437.

[138] Alexander Radovic, "NOvA internal document: Near Detector Data MC Comparisons." http://nova-docdb.fnal.gov:8080/cgi-bin/ShowDocument?docid=15396.

[139] Jianming Bian, Evan Niner, and Kanika Sachdev, "NOvA internal document: LID and e/piO Identifier technote." http://nova-docdb.fnal.gov:8080/cgi-bin/ShowDocument?docid=15344.

[140] F. Dydak, G. Feldman, C. Guyot, J. Merlo, H. Meyer, et al., "A Search for Muon-neutrino Oscillations in the $\Delta \mathrm{m}^{2}$ Range $0.3 \mathrm{eV}^{2}$ to $90 \mathrm{eV}^{2}$," Phys. Lett. B134 (1984) 281-286.

[141] CCFR, I. Stockdale et al., "Limits on Muon-Neutrino Oscillations in the Mass Range 30 i $\Delta m^{2}$; $1000 \mathrm{eV}^{2} / \mathrm{c}^{4}$," Phys. Rev. Lett. 52 (1984) 1384.

[142] SciBooNE, G. Cheng et al., "Dual baseline search for muon antineutrino disappearance at $1 \mathrm{eV}^{2} ; \Delta \mathrm{m}^{2}$; $100 \mathrm{eV} \mathrm{V}^{2}$," Phys. Rev. D86 (2012) 052009, arXiv: 1208.0322.

[143] Christopher J Backhouse, Alexander Himmel, "Nue 2017 Executive Summary." https:

//nova-docdb.fnal.gov/cgi-bin/private/ShowDocument?docid=22475.

[144] Kanika Sachdev, "NOvA internal document: MRCC Technical Note." http://nova-docdb.fnal.gov:8080/cgi-bin/ShowDocument?docid=9729.

[145] Joseph Lozier, "NOvA internal document: ModularExtrap Technical Note." http://nova-docdb.fnal.gov:8080/cgi-bin/ShowDocument?docid=12563. 



\section{List of Publications}

\section{Thesis Publications}

1. P. Adamson et.al. [NOvA Collaboration], "First measurement of electron neutrino appearance in NOvA", Phys. Rev. Lett. 116, 151806 (2016)

2. P. Adamson et.al. [NOvA Collaboration], "First measurement of muon-neutrino disappearance in NOvA", Phys. Rev. D 93051104 (2016)

3. P. Adamson et.al. [NOvA Collaboration], "Constraints on Oscillation Parameters from $\nu_{e}$ Appearance and $\nu_{\mu}$ Disappearance in NOvA", Phys. Rev. Lett. 118, 231801 (2017)

4. P. Adamson et.al. [NOvA Collaboration], "Measurement of the neutrino mixing angle $\theta_{23}$ in NOvA", Phys. Rev. Lett. 118, 151802 (2017)

5. P. Adamson et.al. [NOvA Collaboration], "Search for active-sterile neutrino mixing using neutral-current interactions in NOvA", Phys. Rev. D 96, 072006 (2017), arXiv:1706.04592

6. NOvA Collaboration, "Sterile neutrino search with NOvA Near Detector", to be submitted in mid 2018 to Physical Review Letters .

\section{Other Publications}

1. Bindu A. Bambah, C. Mukku, T. Shreecharan, K. Siva Prasad, "Entanglement in a model for Hawking radiation: An application of quadratic algebras", Annals of Physics, 330, 201-219 (2013). 


\section{Conference Proceedings}

1. Siva Prasad K, Adam Aurisano, Louise Suter, Alex Sousa, Bindu A Bambah, "Search for short-baseline oscillations at the NOvA Near Detector", Proceedings from Neutrino 2016, J.Phys.Conf.Ser. 888 (2017) no.1, 012144, FERMILABCONF-16-426-ND-PPD

2. G.S. Davies, A. Aurisano, G.K. Kafka, S. Kasetti, R. Keloth, A. Sousa, L. Suter and S. Yang, "Searches for Sterile Neutrinos with NOvA", ICHEP 2016 Proceedings, https://pos.sissa.it/282/972/pdf

3. A. Hatzikoutelis, S. Kotelnikov, B. A. Bambah, S. P. Kasetti, "New light weaklycoupled particle searches in a neutrino detector". IOP Journal of Physics: Conference Series 490 (2014) 012070

4. A. Hatzikoutelis, S. Kotelnikov, B. A. Bambah, S. P. Kasetti, "Search for Hidden Sector and Dark Matter Particles Produced at Fermilab's NuMI Target", Proceedings from 10th Patras Workshop on Axions, WIMPs (AXION-WIMP 2014) Conference. FERMILAB-CONF-14-376-PPD 


\section{SEARCH FOR SHORT- BASELINE OSCILLATIONS AT THE NOvA NEAR DETECTOR by Siva Prasad K}

Submission date: 11-Apr-2018 03:44PM (UTC+0530)

Submission ID: 944856026

File name: Siva_12PHPH17_PhD_Thesis_11Apr2018.pdf (14M)

Word count: 40289

Character count: 176614 
SEARCH FOR SHORT-BASELINE OSCILLATIONS AT THE NOVA NEAR DETECTOR

ORIGINALITY REPORT

8.

SIMILARITY INDEX

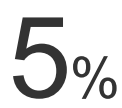

INTERNET SOURCES
$5 \%$

PUBLICATIONS
$1 \%$

STUDENT PAPERS

PRIMARY SOURCES

1 nova-docdb.fnal.gov

Internet Source

2 Submitted to University of Hyderabad, Hyderabad

3 minos-docdb.fnal.gov

Internet Source

4 conservancy.umn.edu

5 P Vahle. "New results from NOvA", Journal of Physics: Conference Series, 2017

6 A Aurisano, C Backhouse, R Hatcher, N Mayer, J Musser, R Patterson, R Schroeter, A Sousa. "

The NO A simulation chain ", Journal of

Physics: Conference Series, 2015

Publication

Siva Prasad Kasetti, Adam Aurisano, Louise 
This is to certify that the thesis entitled "Search For Short-Baseline Oscillations at the NOvA Near Detector" has been screened by the Turnitin software at the library of University of Hyderabad. The software shows only $8 \%$ similarity index. Therefore, this thesis is free from plagiarism.

Prof. Bindu A Bambah

(Thesis Supervisor ) 